\title{
High Frequency Ultrasonic Wave Propagation in Anisotropic Materials
}

\author{
Andrew Paul Dawson
}

A thesis submitted to the Victoria University of Wellington

for the degree of Doctor of Philosophy in Physics

School of Chemical and Physical Sciences

June 2010 


\section{Abstract}

The influence of highly regular, anisotropic, microstructured materials on high frequency ultrasonic wave propagation was investigated in this work. Microstructure, often only treated as a source of scattering, significantly influences high frequency ultrasonic waves, resulting in unexpected guided wave modes. Tissues, such as skin or muscle, are treated as homogeneous by current medical ultrasound systems, but actually consist of highly anisotropic micron-sized fibres. As these systems increase towards $100 \mathrm{MHz}$, these fibres will significantly influence propagating waves leading to guided wave modes. The effect of these modes on image quality must be considered. However, before studies can be undertaken on fibrous tissues, wave propagation in more ideal structures must be first understood.

After the construction of a suitable high frequency ultrasound experimental system, finite element modelling and experimental characterisation of high frequency $(20-200 \mathrm{MHz})$ ultrasonic waves in ideal, collinear, nanostructured alumina was carried out. These results revealed interesting waveguiding phenomena, and also identified the potential and significant advantages of using a microstructured material as an alternative acoustic matching layer in ultrasonic transducer design. Tailorable acoustic impedances were achieved from 4-17 MRayl, covering the impedance range of 7-12 MRayl most commonly required by transducer matching layers. Attenuation coefficients as low as $3.5 \mathrm{dBmm}^{-1}$ were measured at $100 \mathrm{MHz}$, which is excellent when compared with $500 \mathrm{dBmm}^{-1}$ that was measured for a state of the art loaded epoxy matching layer at the same frequency. Reception of ultrasound without the restriction of critical angles was also achieved, and no dispersion was observed in these structures (unlike current matching layers) until at least $200 \mathrm{MHz}$.

In addition, to make a significant step forward towards high frequency tissue characterisation, novel microstructured poly(vinyl alcohol) tissue-mimicking phantoms were also developed. These phantoms possessed acoustic and microstructural properties representative of fibrous tissues, much more realistic than currently used homogeneous phantoms. The attenuation coefficient measured along the direction of PVA alignment in an example phantom was 8 $\mathrm{dBmm}^{-1}$ at $30 \mathrm{MHz}$, in excellent agreement with healthy human myocardium. This method will allow the fabrication of more realistic and repeatable phantoms for future high frequency tissue characterisation studies. 


\section{Acknowledgements}

Firstly, I would like to warmly and heartily thank the efforts of my two supervisors Paul Harris and Gideon Gouws for their continual support and commitment to me throughout this investigation. I will always remember the many hours of discussion spent both local and abroad, not just relating to my research, but the many other interesting problems and thoughts encountered in life. Their light hearted and practical approach to finding solutions made this work very enjoyable.

To the entire Sensors and Electronics team at Industrial Research Limited, this thesis would not have been possible without all of the many forms of support they gave to me. Special thanks to Dale Randall for sorting out all of my flights, conference bookings, formatting my documents, and putting up with my many other nagging requests. To David Greager, Dick Van Den Berg, Rob Kemp, Frederic Lecarpentier, Russell Petherick, and Graeme Woollett I offer a very warm thank you for all of their expert technical support. They have certainly improved my mindset and approach to problems, making me a lot more practical from when I originally started.

I am grateful to Marc Mulholland and Hokmeng Ung from the mechanical development workshop. Their innovative and creative abilities to produce unique, practical solutions to endless complicated and difficult problems will never cease to amaze me.

To my dear Mum and Dad, I owe them my deepest gratitude for their endless emotional and financial support throughout all my studies. Without their assistance, care, and sacrifices they have made for all my education, none of this would have been possible. Thanks to my Dad for feeding my initial interest in electronics, showing me his yellowed electrical schematics, his and Grandad's old dusty books, and his old projects. Cheers to my Mum for all the home baking that was not only always enjoyed and appreciated by me but also the majority of the Engineering and Applied Physics team.

Finally, but certainly not least, I would like to express my gratitude to my brother in law Wade Murphy for feeding my initial interest in science. The Usbourne Book of Physics he purchased for my $11^{\text {th }}$ birthday set my path early for a career in science. 


\section{List of Conference Presentations and Publications}

- A. Dawson, P. Harris, G. Gouws, "Design and Evaluation of Ultrasonic Transducer Circuits for Material Characterisation," in Proc. 13 $3^{\text {th }}$ Electronics New Zealand Conference, Christchurch, New Zealand, pp 261-265, 2006.

- P. Harris, A. Dawson, R. Young, F. Lecarpentier, "High Frequency Propagation in Structured Solids," in Proc. IEEE International Ultrasonics Symposium, N.Y, USA, pp 690693, 2007.

- A. Dawson, P. Harris, G. Gouws, "High Frequency Ultrasound System for Analysis of Anisotropic Materials," in Proc. 14 $4^{\text {th }}$ Electronics New Zealand Conference, Wellington, New Zealand, pp 137-140, 2007.

- A. Dawson, P. Harris, G. Gouws, "High Frequency Ultrasound Wave Propagation in Anisotropic Materials," in Proc. IEEE $2^{\text {nd }}$ International Conference on Sensing Technology, Palmerston North, New Zealand, pp 552-556, 2007.

- A. Dawson, P. Harris, G. Gouws, "High Frequency Wave Propagation in Porous Aluminum Samples," Smart Sensors and Sensing Technology, Springer-Verlag, Berlin, pp 221-232, 2008.

- R. Young, P. Harris, A. Dawson, F. Lecarpentier, "High Frequency Wave Propagation in Structured Materials: Modeling Results," in Proc. IEEE International Ultrasonics Symposium, Beijing, China, pp 532-535, 2008.

- A. Dawson, P. Harris, R. Young, G. Gouws, "High Frequency Propagation Measurements in Structured Solids," in Proc. IEEE International Ultrasonics Symposium, Beijing, China, pp 74-77, 2008.

- A. Dawson, P. Harris, G. Gouws, "High Frequency Ultrasound Propagation in Microstructured Solids," in Proc. $15^{\text {th }}$ Electronics New Zealand Conference, Auckland, New Zealand, pp 147-152, 2008.

- A. Dawson, International PZFlex Student innovation Competition WINNER, March, 2009.

- P. Harris, A. Dawson, R. Young, "Microstructured Materials - Propagation and use for Impedance Matching," in Proc. IEEE International Ultrasonics Symposium, Rome, Italy, pp. 1165-1168, 2009.

- A. Dawson, P. Harris, G. Gouws, "Preparation and Characterisation of Microstructured Poly(Vinyl Alcohol) Phantoms Mimicking Fibrous Tissue," in Proc. IEEE International Ultrasonics Symposium, Rome, Italy, pp. 1958-1961, 2009.

- A. Dawson, P. Harris, R. Young, G. Gouws, "Acoustic Impedance Matching using Porous Aluminium," in Proc. IEEE $8^{\text {th }}$ International Conference on Sensors, Christchurch, New Zealand, pp. 49-52, 2009. 
- R. Young, A. Dawson, P. Harris, "Modelling of High Frequency Wave Propagation in Structured Materials," IEEE Transactions on Ultrasonics, Ferroelectrics, and Frequency Control, (accepted for publication), 2010.

- A. Dawson, P. Harris, G. Gouws, "Anisotropic Microstructured Poly(Vinyl Alcohol) Tissue Mimicking Phantoms," IEEE Transactions on Ultrasonics, Ferroelectrics, and Frequency Control, Vol. 57, No. 7, pp. 1494-1496, 2010.

- A. Dawson, P. Harris, "Freeze Casted Microstructured PVA Tissue Phantoms Using a Microstructured Seed Layer," in Proc. IEEE International Ultrasonics Symposium, San Diego, USA, (to be presented), Oct, 2010. 


\section{Table of Contents}

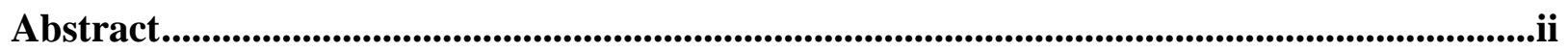

Acknowledgements ......................................................................................................................................... iii

List of Publications .......................................................................................................................................iv

Table of Figures....................................................................................................................................ix

1. Introduction............................................................................................................................................1

2. Theoretical Background .......................................................................................................5

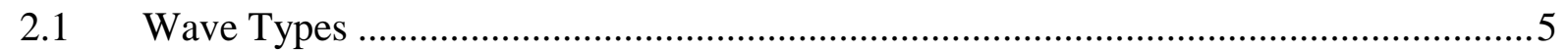

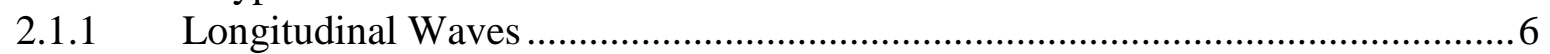

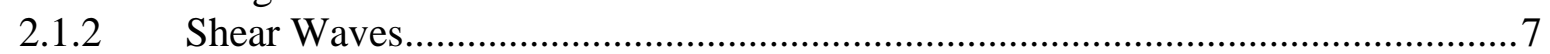

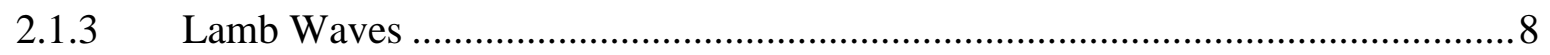

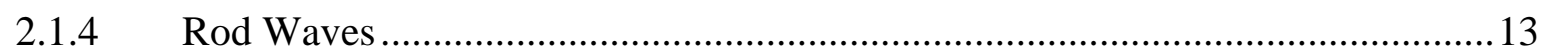

2.1.5 Rayleigh \& Interface Waves .......................................................................16

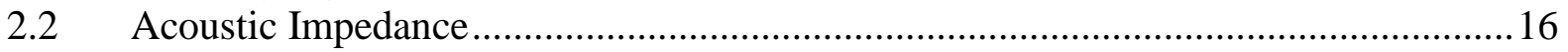

2.3 Transmission and Reflection in Homogeneous Media .................................................17

2.3.1 Fluid-Solid Interface: Normal Incidence ………............................................17

2.3.2 Fluid-Solid Interface: Oblique Incidence....................................................19

2.3.3 $\quad \lambda / 4$ Wavelength Matching Layers.................................................................24

2.4 Wave Propagation in Anisotropic Media............................................................26

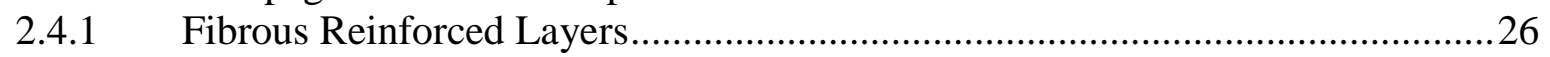

2.4.2 Collinear Porous Solids..................................................................................28

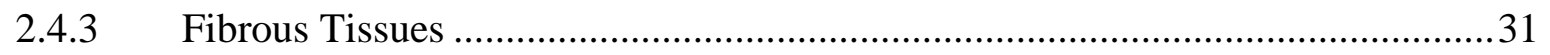

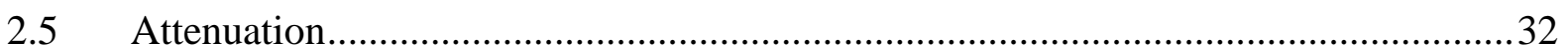

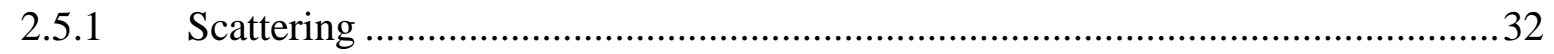

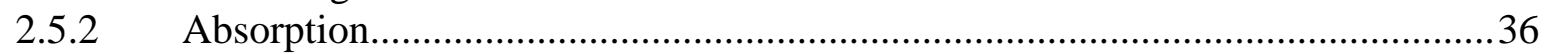

3. Design and Setup of Experimental Systems ……………….........................................................39

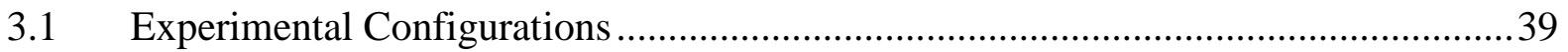

3.1.1 Transmission Measurement System …………………………………..........39

3.1.2 Pulse Echo Measurement System ...................................................................

3.2 High Frequency Ultrasonic Transducer Characteristics .............................................46

3.2.1 Piezoelectric Transducer Construction ............................................................46

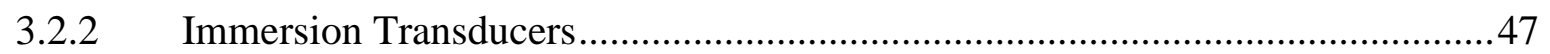

3.2.3 Transducer Frequencies ..........................................................................4

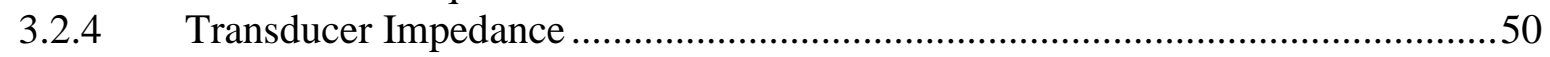

3.2.5 Transducer Beam Properties .............................................................................54

3.3 Development of High Frequency Electronics ............................................................58

3.3.1 Board Layout Considerations …………………........................................59

3.3.2 $100 \mathrm{MHz}$ Transmitter...................................................................................59

3.3.3 $100 \mathrm{MHz}$ Receiver ................................................................................... 
3.3.4 $1000 \mathrm{MHz}$ Minicircuits 20W Power Amplifier ..............................................67

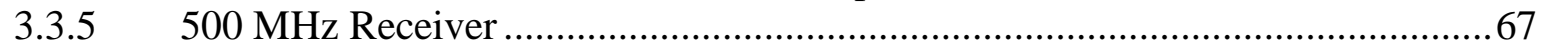

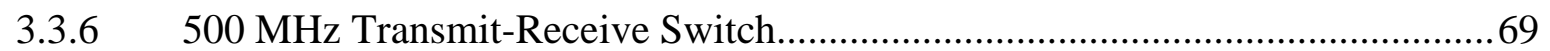

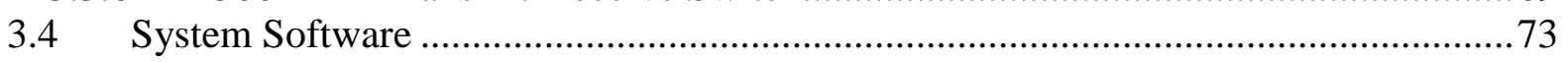

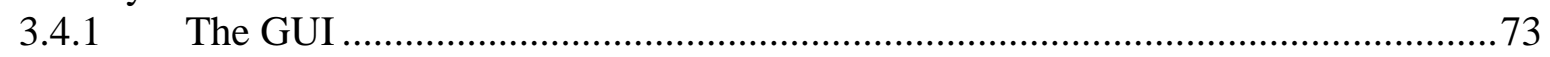

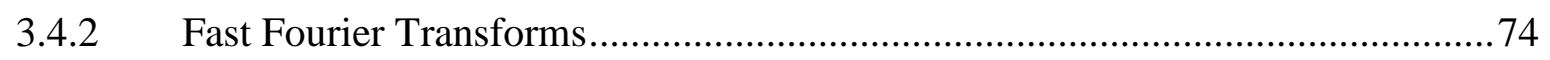

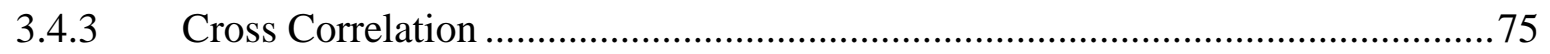

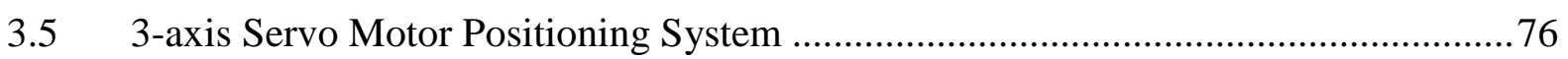

4. Porous Anodic Alumina (PAA) Matching Layers - Fabrication and Structure ..............77

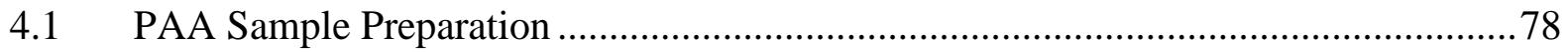

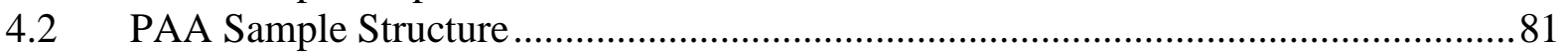

5. Finite Element Modelling of Collinear Porous Solids ..................................................87

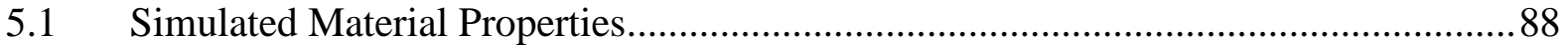

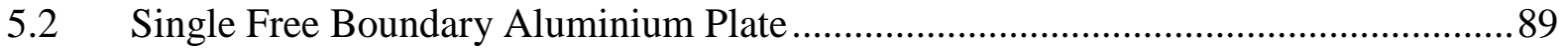

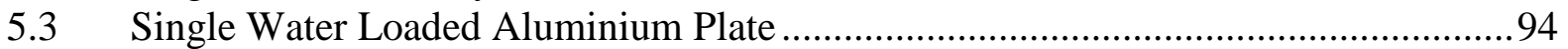

5.4 Water Filled Porous Aluminium............................................................................ 98

5.5 Normalisation and Preservation of Waveforms in Porous Aluminium Layers .........105

5.6 Acoustic Impedance of Free Boundary Aluminium Plate ........................................ 109

5.7 Rectangular and Triangular Architecture Models of PAA Samples.......................... 114

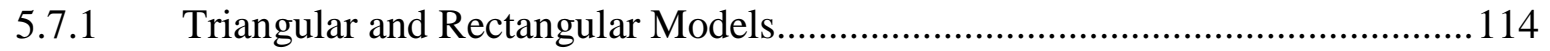

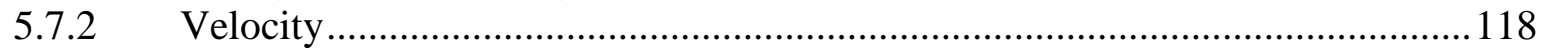

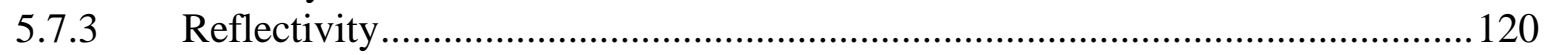

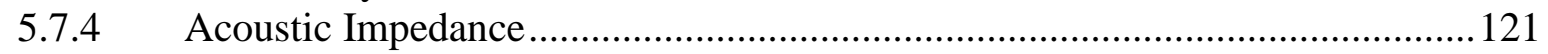

5.8 Significance of the Modelling Results - PAA Transducer Matching Layers .............123

6. Experimental Characterisation of PAA Samples ........................................................................125

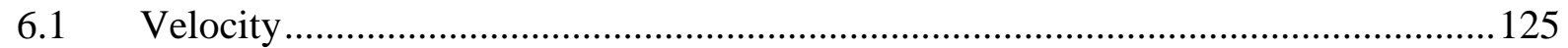

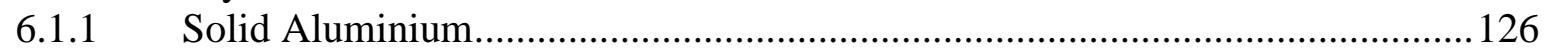

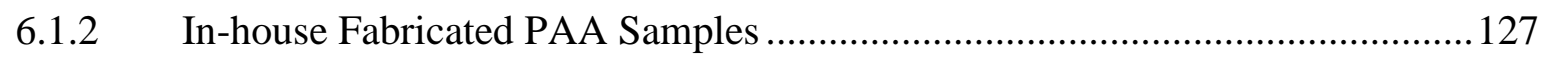

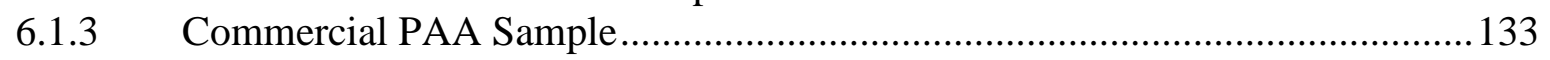

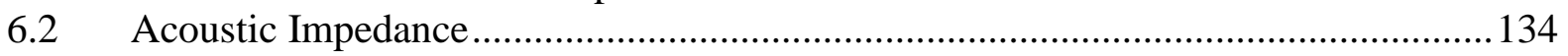

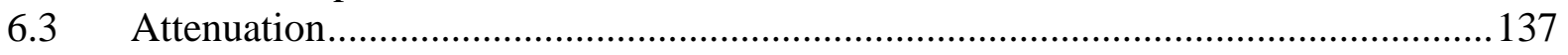

6.4 Summary of Measured PAA Sample Properties..................................................... 139

7. Anisotropic Poly(Vinyl-Alcohol) Tissue Mimicking Phantoms .....................................141

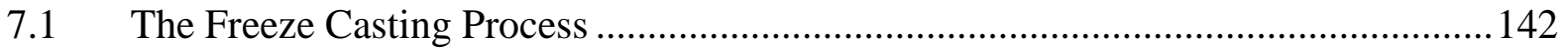

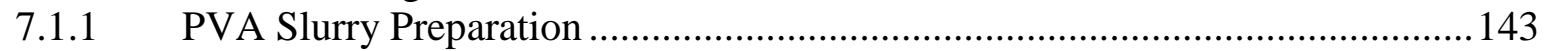

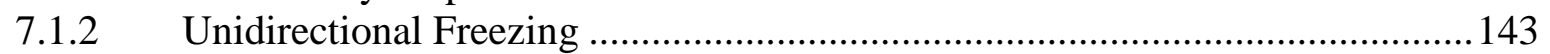

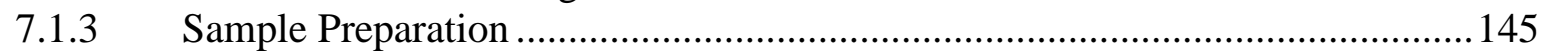

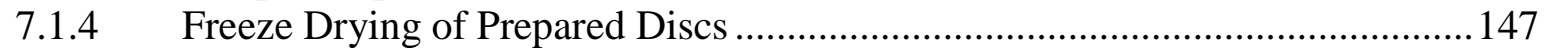

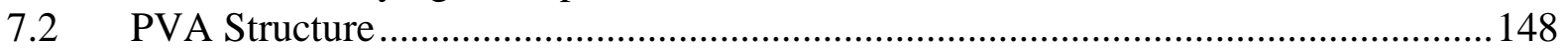

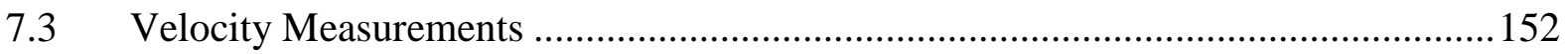

7.4 Attenuation Measurements ................................................................................ 154 
8.1 Finite Element Modelling of High Frequency Ultrasonic Wave Propagation in Microstructured Materials

8.2 Experimental Characterisation of Porous Anodic Alumina Samples for Use As

Acoustic Matching Layers

8.3 Microstructured Poly(Vinyl Alcohol) Tissue Mimicking Phantoms..........................161

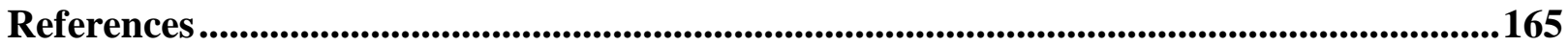

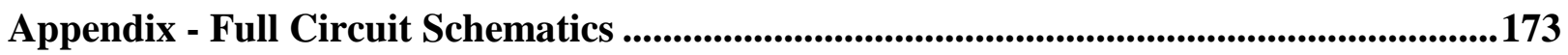

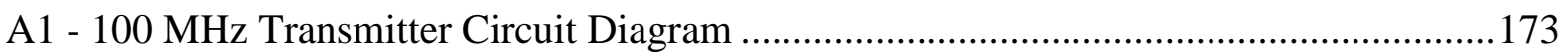

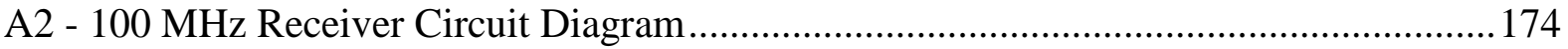

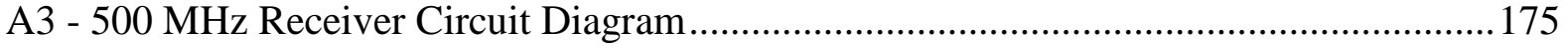

A4 - 500 MHz Transmit/Receive Switch Circuit Diagram ............................................... 176 


\section{Table of Figures}

Figure 2.1 - Grid diagram showing the deformation of a solid caused by a propagating longitudinal wave at four instances of time [18].

Figure 2.2 - Grid diagram showing the deformations of a solid caused by a propagating shear wave at four instances of time [18]

Figure 2.3 - Generation of a Lamb wave in a plate caused by the interactions of multiple $\mathrm{P}$ (solid lines) and S (broken lines) waves [24]. The different angles of refraction are due to the different speeds of the $\mathrm{P}$ and $\mathrm{S}$ waves.

Figure 2.4 - Lamb wave phase velocity dispersion curves for a free boundary aluminium plate for both symmetric modes (solid lines) and antisymmetric modes (broken lines) [19]..... 10

Figure 2.5 - Lamb wave group velocity dispersion curves for a free boundary aluminium plate for both symmetric modes (solid lines) and antisymmetric modes (broken lines) [19]......12

Figure 2.6 - Plate displacement shown by the circular arrows for $S_{0}$ (top) and $A_{0}$ (bottom) Lamb

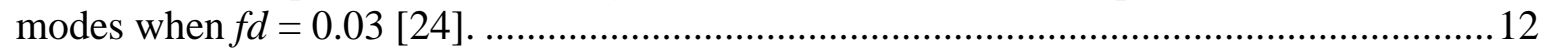

Figure 2.7 - Longitudinal mode phase velocity dispersion curves for a free boundary aluminium $\operatorname{rod}[19]$.

Figure 2.8 - Longitudinal mode group velocity dispersion curves for a free boundary aluminium $\operatorname{rod}[19]$.

Figure 2.9 - Normally incident pressure wave reflected and transmitted at media $Z_{1} / Z_{2}$ interface.

Figure 2.10 - Obliquely incident pressure wave reflected and refracted at a liquid-solid interface.

Figure 2.11 - Variation in the magnitude of the pressure reflection coefficient with angle of incidence at a water-aluminium interface.

Figure 2.12 - Percentage energy reflected and transmitted as shear or longitudinal waves at a water-aluminium interface.

Figure 2.13 - Phase velocity dispersion curves for a unidirectional fibre reinforced composite plate for propagation parallel to the direction of fibre alignment [19].

Figure 2.14 - Phase velocity dispersion curves for a unidirectional fibre reinforced composite plate for propagation perpendicular to the direction of fibre alignment [19] . ...................27

Figure 2.15 - Examples of the different types of fibre reinforced composites.........................28

Figure 2.16 - Longitudinal cross section of a porous anodic alumina sample. The pores extend from the top of the sample to the bottom with no irregularities. The sample was physically snapped in half giving rise to the observed broken pores.

Figure 2.17 - Myocardium tissue sample from a human heart that demonstrates a fibrous anisotropic microstructure.

Figure 2.18 - Polar diagrams showing scattering from a rigid sphere for $k a$ values of (a) 5, (b)

3 , and (c) $1.180^{\circ}$ corresponds to reflections directed back at the source, and $0^{\circ}$

corresponds to propagation directly away from the source [67].

Figure 2.19 - Total scattered power from a sphere of radius $a$ versus $k a$ number. ....................35

Figure 2.20 - Acoustic absorption in fresh water versus frequency.......................................37

Figure 3.1 - Transmission measurement experimental configuration.......................................40

Figure 3.2 - Transmission measurement water bath setup.................................................41

Figure 3.3 - Time of flight measurements (a) without sample, and (b) with sample in place....41

Figure 3.4 - Phase measurements to calculate sample velocity for known sample thickness $d .42$

Figure 3.5 - Amplitude measurements to calculate sample attenuation coefficient. .................43

Figure 3.6 - Pulse echo measurement experimental configuration.........................................44 
Figure 3.7 - Pulse echo measurements for unknown sample acoustic impedance. ..................45

Figure 3.8 - Cross section of a contact piezoelectric transducer [76] ....................................47

Figure 3.9 - Typical frequency response for the V390 $50 \mathrm{MHz}$ transducers [85]...................48

Figure 3.10 - Typical frequency response for the V3802 $110 \mathrm{MHz}$ transducers [85]..............49

Figure 3.11 - Typical frequency response for the V3965 $175 \mathrm{MHz}$ transducers [85]..............49

Figure 3.12 - Magnitude (a) and phase (b) characteristics of the two $50 \mathrm{MHz}$ transducers.......51

Figure 3.13 - Magnitude (a) and phase (b) characteristics of the two $110 \mathrm{MHz}$ transducers.....52

Figure 3.14 - Magnitude (a) and phase (b) characteristics of the two $175 \mathrm{MHz}$ transducers.....53

Figure 3.15 - Beam diameter, focal length, and focal zone properties of a transducer [77]......55

Figure 3.16 - Transducer beam diameter versus frequency for $50 \mathrm{MHz}, 110 \mathrm{MHz}$, and 175

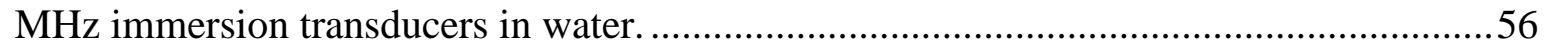

Figure 3.17 - Focal zone length with frequency for the $50 \mathrm{MHz}, 110 \mathrm{MHz}$, and $175 \mathrm{MHz}$

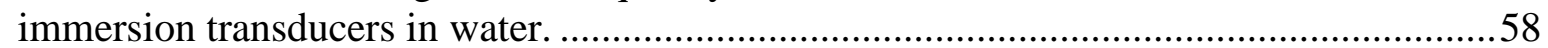

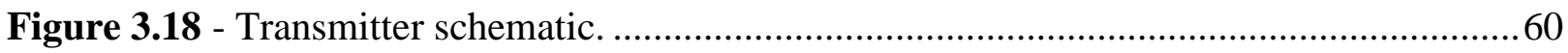

Figure 3.19 - Transmitter gain versus frequency. ...........................................................6 60

Figure 3.20 - $100 \mathrm{MHz}$ receiver schematic...................................................................6 62

Figure 3.21 - Receiver gain versus frequency for five different gains from minimum gain of 17

$\mathrm{dB}$ (lowest trace) to maximum gain of $65 \mathrm{~dB}$ (highest trace).......................................65

Figure 3.22 - Receiver output noise (top) and spectrum analyser noise (bottom) traces...........65

Figure 3.23 - A time gain compensated waveform shows an exponential rise, with the square wave SYNC signal resetting the integrator ramp to zero, and thus stopping the time gain compensation.

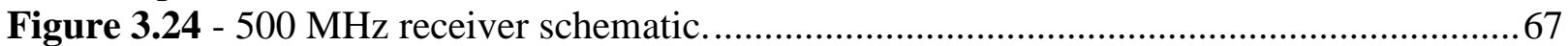

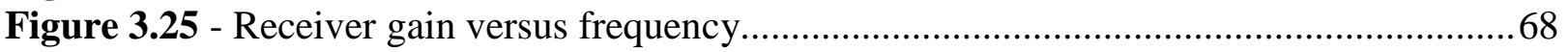

Figure 3.26 - Receiver circuit and spectrum analyser noise traces. ......................................68

Figure 3.27 - Simplified transmit-receive switch schematic.............................................. 70

Figure 3.28 - Transmit-receive switch circuit receiver gain with frequency. ..........................72

Figure 3.29 - Transmit-receive switch receiver off characteristic with frequency...................72

Figure 3.30 - System GUI displaying data captured from $\mathrm{CH} 1$ and $\mathrm{CH} 2$ of the oscilloscope...74

Figure 3.31 - Magnitudes of FFTs performed on $\mathrm{CH} 1$ and $\mathrm{CH} 2$ data...................................75

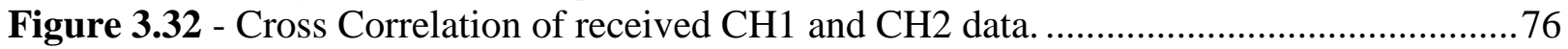

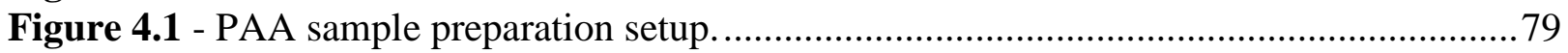

Figure 4.2 - PAA interpore distance variations with anodising voltage for the sulphuric, oxalic,

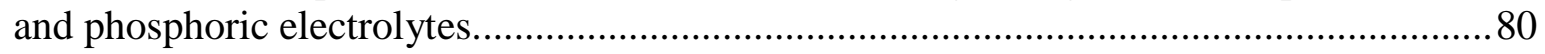

Figure 4.3 - Horizontal cross section of a sulphuric acid prepared porous alumina sample (type 1 sample) showing $30 \mathrm{~nm}$ sized pores with $60 \mathrm{~nm}$ spacing.......................................... 82

Figure 4.4 - Longitudinal cross section of a sulphuric acid prepared porous alumina sample (type 1 sample) showing the highly collinear structure of these samples...........................82

Figure 4.5 - Horizontal cross section of an oxalic acid prepared porous alumina sample (type 2

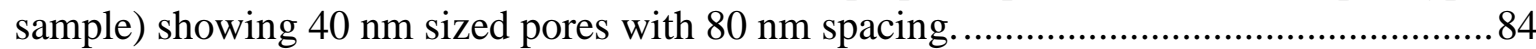

Figure 4.6 - Horizontal cross section of a phosphoric acid prepared porous alumina sample (type 3 sample) showing on average $400 \mathrm{~nm}$-sized pores with $500 \mathrm{~nm}$ spacing.................84

Figure 4.7 - Horizontal cross section of commercial porous alumina sample ...........................85

Figure 4.8 - Longitudinal cross section of commercial porous alumina sample. .....................85

Figure 5.1 - Finite element model of a single free boundary aluminium plate with boundary

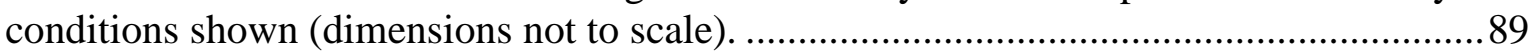

Figure 5.2 - Blackman pulse in time and frequency domains...............................................90

Figure 5.3 - Group velocity in a free boundary aluminium plate as a function of the frequencythickness product for end on excitation. ..................................................................... 91 
Figure 5.4 - Pulse pressure in the free boundary aluminium plate at (a) the beginning of all plates, and at the end of (b) $1 \mathrm{~mm}$, (c) $5 \mathrm{~mm}$, and (d) $9 \mathrm{~mm}$ thick plates.

Figure 5.5 - Power through a vertical line of plate nodes at the beginning (top left) of all plates, and upon reaching the end of a $1 \mathrm{~mm}$ (top right), $5 \mathrm{~mm}$ (bottom left), and $9 \mathrm{~mm}$ (bottom right) thick plates.

Figure 5.6 - Finite element model of a fluid loaded aluminium plate with boundary conditions

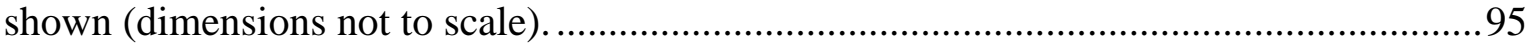

Figure 5.7 - Water loaded aluminium plate snapshots $47 \mu$ s into the simulation for three

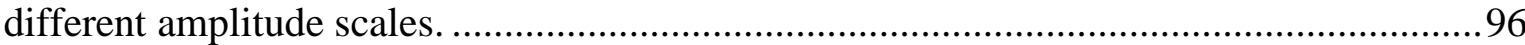

Figure 5.8 - Fast wave velocity in a water loaded aluminium plate with frequency-thickness product.

Figure 5.9 - Slow wave velocity in a water loaded aluminium plate with frequency-thickness product.

Figure 5.10 - 2D model of a water loaded porous aluminium layer connected to solid aluminium with boundary conditions as shown. The source was excited on the bottom most pillar.

Figure 5.11 - Simulation of water loaded porous aluminium connected to solid aluminium (a) $35 \mathrm{~ns}$, (b) $70 \mathrm{~ns}$, and (c) $105 \mathrm{~ns}$ after the beginning of the simulation.

Figure 5.12 - Plane wave excitation of water loaded porous aluminium connected to solid aluminium (a) $39 \mathrm{~ns}$, (b) $71 \mathrm{~ns}$, and (c) $178 \mathrm{~ns}$ after excitation..................................... 102

Figure 5.13 - Fast wave to slow wave energy ratio versus frequency for $2 \mu \mathrm{m}$ aluminium pillar and pore widths.

Figure 5.14 - Fast wave to slow wave energy ratio versus porosity at $50 \mathrm{MHz}$ and $150 \mathrm{MHz}$.

Figure 5.15 - 2D model of a water loaded porous aluminium layer connected to both water and solid aluminium with boundary conditions shown. A single element was excited as shown.

Figure 5.16 - Simulation of spherical wave (a) $35 \mathrm{~ns}$, (b) $70 \mathrm{~ns}$, and (c) $90 \mathrm{~ns}$ after the beginning of the simulation.

Figure 5.17 - 2D model of a single aluminium plate connected to solid aluminium with boundary conditions shown.

Figure 5.18 - Energy integral and instantaneous power plots for a $100 \mathrm{kHz}$ wave in an aluminium plate of $1 \mathrm{~mm}$ thickness. Snapshots are recorded at (a) the beginning of the aluminium plate, (b) halfway along the aluminium plate, and (c) at the beginning of the widened aluminium solid.

Figure 5.19 - Percentage energy reflected from aluminium plate/solid interface with $f d$ product.

Figure 5.20 - Aluminium plate acoustic impedance versus $f d$ product...

Figure 5.21 - Rectangular model horizontal cross section used to model the type 2 and type 3 PAA sample architectures.

Figure 5.22 - Triangular model horizontal cross section used to model the type 1 PAA sample architecture.

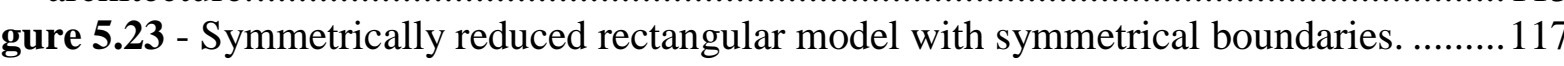

Figure 5.24 - Symmetrically reduced triangular model with symmetrical boundaries............ 117

Figure 5.25 - Rectangular and triangular model velocities versus porosity for 50 and $150 \mathrm{MHz}$ waves.

Figure 5.26 - Rectangular and triangular model energy reflection coefficients versus porosity at 50 and $150 \mathrm{MHz}$.....

Figure 5.27 - Rectangular model acoustic impedance versus porosity at $150 \mathrm{MHz}$. 122 
Figure 6.1 - Variations in group velocity with transducer angle of incidence at $50 \mathrm{MHz}$ for solid

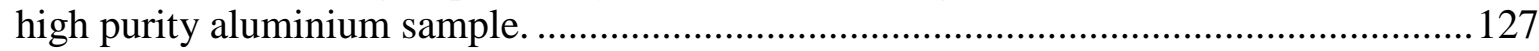

Figure 6.2 - Variations in sample group velocity with transducer angle of incidence at $50 \mathrm{MHz}$

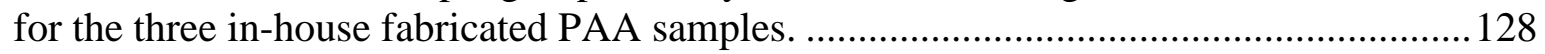

Figure 6.3 - Variations in sample group velocity with transducer angle of incidence at 100 $\mathrm{MHz}$ for the three in-house fabricated PAA samples.

Figure 6.4 - Variations in sample group velocity with transducer angle of incidence at 175 $\mathrm{MHz}$ for the three in-house fabricated PAA samples.

Figure 6.5 - Velocity versus angle of incidence at $50 \mathrm{MHz}, 100 \mathrm{MHz}$, and $175 \mathrm{MHz}$ for the type 1 samples.

Figure 6.6 - Velocity versus angle of incidence at $50 \mathrm{MHz}, 100 \mathrm{MHz}$, and $175 \mathrm{MHz}$ for the type 2 samples.

Figure 6.7 - Velocity versus angle of incidence at $50 \mathrm{MHz}, 100 \mathrm{MHz}$, and $175 \mathrm{MHz}$ for the type 3 samples.

Figure 6.8 - Average sample velocity versus porosity at $50 \mathrm{MHz}, 100 \mathrm{MHz}$, and $175 \mathrm{MHz}$. 132

Figure 6.9 - Variations in group velocity with transducer angle of incidence at $50 \mathrm{MHz}$ for a commercial PAA sample.

Figure 6.10 - Average acoustic impedance of PAA samples versus porosity. ........................136

Figure 6.11 - Variations in PAA sample attenuation with frequency................................138

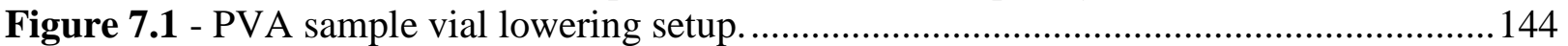

Figure 7.2 - The freeze casting process showing the ice crystal formation within the slurry vial

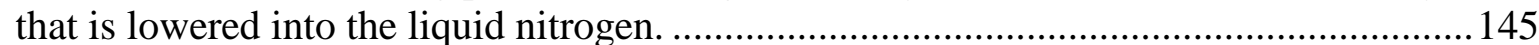

Figure 7.3 - Axial and radial cuts made on frozen PVA samples..........................................146

Figure 7.4 - Pressure/temperature phase transition diagram for pure water........................... 147

Figure 7.5 - Horizontal cross section (section made perpendicular to the direction of freeze casting) of a $5 \%$ concentration by weight PVA phantom lowered at $3 \mathrm{~mm} / \mathrm{min}$.

Figure 7.6 - Longitudinal cross section (section made parallel to the direction of freeze casting) of a $5 \%$ concentration by weight PVA phantom lowered at $3 \mathrm{~mm} / \mathrm{min}$.

Figure 7.7 - Average pore size versus freezing rate for $5 \%$ by weight PVA concentration.... 151

Figure 7.8 - Phantom velocity variations with frequency for a $7 \%$ concentration by weight PVA phantom measured parallel (circles) and perpendicular (squares) to the direction of freeze casting.

Figure 7.9 - Attenuation coefficient versus frequency for $7 \%$ concentration by weight PVA phantom (circles) measured along the direction of PVA alignment (freeze casting direction). Also plotted are measurements made parallel to the fibre alignment on normal human myocardium tissue (filled squares) obtained from [127]. 


\section{Chapter 1}

\section{Introduction}

Microstructure in materials is often only considered as a source of scattering for incident ultrasonic waves. However, a fibrous or porous microstructure can have a profound influence on high frequency ultrasonic wave propagation, resulting in unexpected guided wave modes. These occurrences of guided waves will become more apparent in the future as transducer technology continues to improve and higher frequency devices are realised.

Consideration of microstructure is important for both medical ultrasound and ultrasonic nondestructive testing (NDT). In medical ultrasound, tissues are usually treated as a homogeneous material, where only the bulk acoustic properties of the media are considered [1]-[3], [40], [64]. However, when viewed at micron length scales tissues are found to comprise of microstructure. Tissues such as collagen fibres [4]-[7] possess lengths of 50-100 $\mu \mathrm{m}$ that are an order of magnitude greater than their respective diameters. These fibres consist of sub-micron fibrils that possess diameters of hundreds of nanometers. In healthy tissues these fibres are oriented along the same direction, displaying significant anisotropy in a layered like structure. When these tissues develop defects, for example due to medical conditions [8]-[10], the orientation and alignment can become disrupted modifying the surrounding structures, with the generated acoustic signatures dependent upon the local tissue structure. 
Tissue then should be seen as a multi-scale medium containing microstructure. For example the dermis layer in the skin at current imaging frequencies of 5-20 $\mathrm{MHz}$ appears highly echogenic (uniformly reflective) [11], while at $100 \mathrm{MHz}$ and higher frequencies is expected to be locally anisotropic, both a consequence of the collagen and its microstructure. As the ability to fabricate higher frequency transducers continues to improve medical imaging equipment manufacturers attempt to provide improved resolution [12]-[14]. However it is believed there will become a point when little is achieved unless the underlying microstructures are considered. The incident wavelengths will become comparable to the underlying tissue microstructure dimensions, influencing the wave velocity, scattering, and number of waves observed.

Although fibrous tissues are highly anisotropic, they do contain irregularities in their microstructure. Therefore a solid understanding of wave propagation in ideal anisotropic structures is essential before the high frequency characterisation of more complex fibrous tissues is undertaken.

Performing measurements on real tissues also leads to complications involving difficulties with sample preparation, problems with consistency between samples during long term studies, and ethical issues regarding the use of real tissues. Homogeneous tissue mimicking materials (or phantoms) currently used by researchers [113]-[116] reflect the basic acoustic properties of real tissues but fail to incorporate the anisotropic microstructure and dimensions needed to investigate the wave guiding properties discussed. The need for more realistic tissue phantoms that replicate both the acoustic and microstructural properties of real tissues are therefore essential to understand and advance the use of higher frequencies in medical ultrasound.

A second application area of high frequency ultrasound where a consideration of the material substructure can lead to significant advances is found in the non-destructive testing [15], [16] of highly regular microstructured and nanostructured solids. Microstructure can occur unexpectedly in materials believed to be homogeneous. For example, solid aluminium readily oxidises at its interface [17] leading to a highly anisotropic and regular aluminium oxide surface layer (also known as alumina). This anodising property has been taken advantage of for many years leading to rapid advances in the fabrication of porous materials such as porous anodic alumina (or PAA) [87]-[90]. Due to the ideal regularity of the PAA microstructure, the 
propagation of high frequency ultrasound can be significantly influenced by variations in the porous solid structure e.g. changes in dimensions, porosity, architecture etc.

By understanding how wave propagation is influenced by variations in these structural properties the potential exists for porous materials to be precisely tailored to possess acoustic properties required for a specific application e.g. for transducer impedance matching layers [33]-[39]. Matching layers are an important component of medical ultrasound transducers, increasing the energy transfer from the transducer active element into the sample of interest. As frequencies continue to increase above $100 \mathrm{MHz}$, these layers need to be as acoustically efficient as possible [103], since maximising energy transfer becomes critical due to the significant attenuation at such high frequencies.

The consideration of material microstructure can thus lead to significant benefits in both ultrasonic transducer design and diagnostic medical ultrasound. The ideal properties of the PAA samples mentioned above are not only potentially useful for transducer design, but also provide an excellent basis for the understanding of wave propagation in other highly anisotropic materials. They possess nanometer sized dimensions of the order of collagen microfibrils that form the building block of the larger collagen fibres. Armed with this more simplified understanding from the PAA samples wave propagation in more challenging fibrous tissue microstructures can be investigated in future studies. In order to make a contribution to both of these areas the following work was undertaken:

- The design and construction of specialised instrumentation for the experimental measurement of high frequency ultrasonic waves interacting with sample materials (Chapter 3). This included transducers, custom low noise and wide bandwidth transmitter/receiver electronics, system software, and apparatus.

- The fabrication of physical PAA samples prepared by anodising high purity aluminium (Chapter 4). Three different sample types were fabricated possessing ideal regularity and collinearity with variable nanometer sized pores/pore spacing, porosities, and architectures in order to create a range of these properties, with the effects of these properties characterised experimentally and compared with modelling results.

- Finite element modelling of high frequency ultrasonic wave propagation in highly collinear anisotropic materials (Chapter 5) with properties similar to the fabricated PAA 
samples. With the increasing power of modern personal computers and the availability of dedicated finite element modelling packages, the influences of structural variations in PAA samples could be investigated before the fabrication of the physical samples and experimental work commenced. Modelling was performed on both 2D and 3D structures to examine the wave guiding phenomena in highly structured media, and observe the variations in velocity, reflectivity, and acoustic impedance with changes in the PAA sample structural properties.

- Experimental characterisation of PAA samples using the designed high frequency ultrasound system (Chapter 6). These measurements were performed to experimentally verify the wave guiding phenomena observed in the modelling, and reveal other nonmodelled physical properties such as attenuation coefficients.

- Development of novel tissue phantoms using a unique freeze casting process to be used as more realistic tissue mimicking materials in the understanding of the waveguiding influence of anisotropic fibrous tissues on high frequency ultrasound (Chapter 7). The process results in the fabrication of repeatable poly(vinyl alcohol) tissue phantoms possessing similar microstructural and acoustic properties as found in real fibrous tissues. These samples were then characterised experimentally with velocity and attenuation measurements performed.

As a result of the above work the two main original and significant contributions to the ultrasound community were:

1.) The concept, modelling, and fabrication of PAA transducer matching materials that possess tailorable acoustic impedances in the range of 4-17 Rayl, allow the transmission of energy beyond $\mathrm{P}$ and $\mathrm{S}$ critical angles, display no measurable dispersion at least to $200 \mathrm{MHz}$, and possess very low attenuation coefficients at very high frequencies e.g. as low as $3.5 \mathrm{dBmm}^{-1}$ at $100 \mathrm{MHz}$.

2.) The fabrication and characterisation of novel anisotropic PVA tissue phantoms that closely resemble and incorporate the structure, dimensions, and acoustic properties of real microstructured tissue. These phantoms provide an excellent building block for the fabrication of more advanced tissue phantoms for higher frequency medical ultrasound research. 


\section{Chapter 2}

\section{Theoretical Background}

This chapter covers the fundamental principles and background of ultrasonic wave propagation. It begins by looking at the fundamental ultrasonic wave types encountered in section 2.1, followed by the important concept of acoustic impedance in section 2.2. Transmission and reflection phenomena in homogeneous media is presented in section 2.3 , recent contributions to the area of ultrasonic wave propagation in anisotropic media are discussed in section 2.4 , and finally attenuation phenomena are presented in section 2.5 .

\subsection{Wave Types}

In this section fundamental wave types of relevance to this work are discussed. These can be separated into two main categories; bulk waves and guided waves. Bulk longitudinal and shear waves propagate in infinite homogeneous media where they are unconstrained by material dimensions. Guided waves propagate when a boundary or a structure of finite dimension relative to the propagating wave wavelength is introduced, forming waves such as interface or plate waves depending on the applied boundary conditions. Guided waves are the superposition of multiple reflections and interactions of longitudinal and shear waves caused by a structural boundary condition. 


\subsubsection{Longitudinal Waves}

Longitudinal waves, also known as compressional or $\mathrm{P}$ waves, are bulk waves that travel at the velocity determined by the elastic properties of a medium. In homogeneous media the polarization of the compressional wave is always longitudinal, that is the particle motion of the solid consists of vibrations parallel to the direction of energy transfer of the wave. Longitudinal waves propagate in both liquids and solids. Figure 2.1 shows the displacements caused by a longitudinal waveform propagating in a homogeneous solid represented by a grid of square elements at four different instants in time.

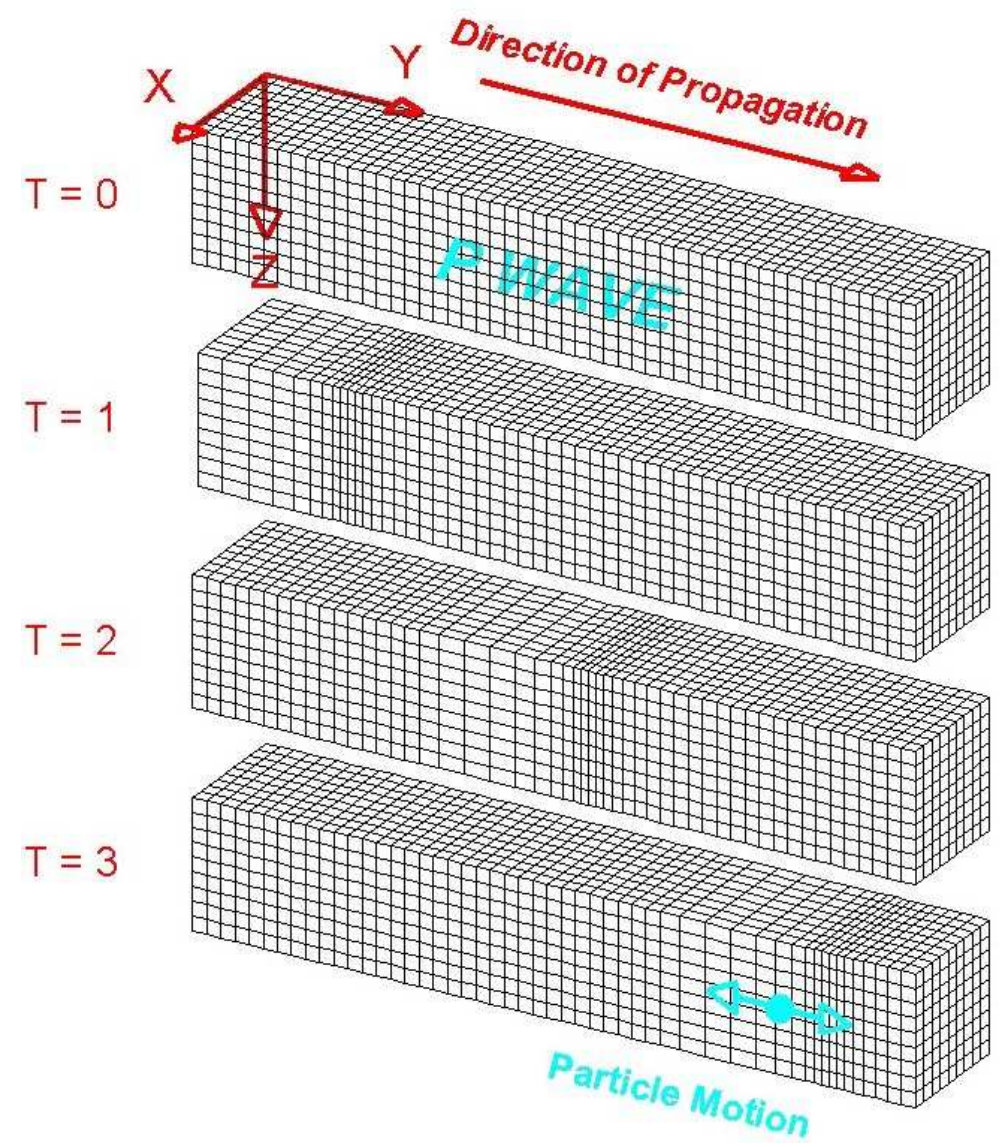

Figure 2.1 - Grid diagram showing the deformation of a solid caused by a propagating longitudinal wave at four instances of time [18].

The propagating wave causes a compression of the grid (where the grid lines are compact) followed by a rarefaction (grid lines are expanded). As can be observed the grid or particle motion of the solid is along the direction of propagation. 


\subsubsection{Shear Waves}

Shear waves, also known as transverse or $\mathrm{S}$ waves, are the second type of bulk wave where the polarization or particle motion is perpendicular to the direction of energy transfer. Shear waves only propagate in solids, as liquids cannot support shear. $\mathrm{S}$ waves travel with a velocity typically around half the $\mathrm{P}$ wave velocity in the same medium. Two types of polarisation are possible; shear vertical (SV waves), or shear horizontal (SH waves).

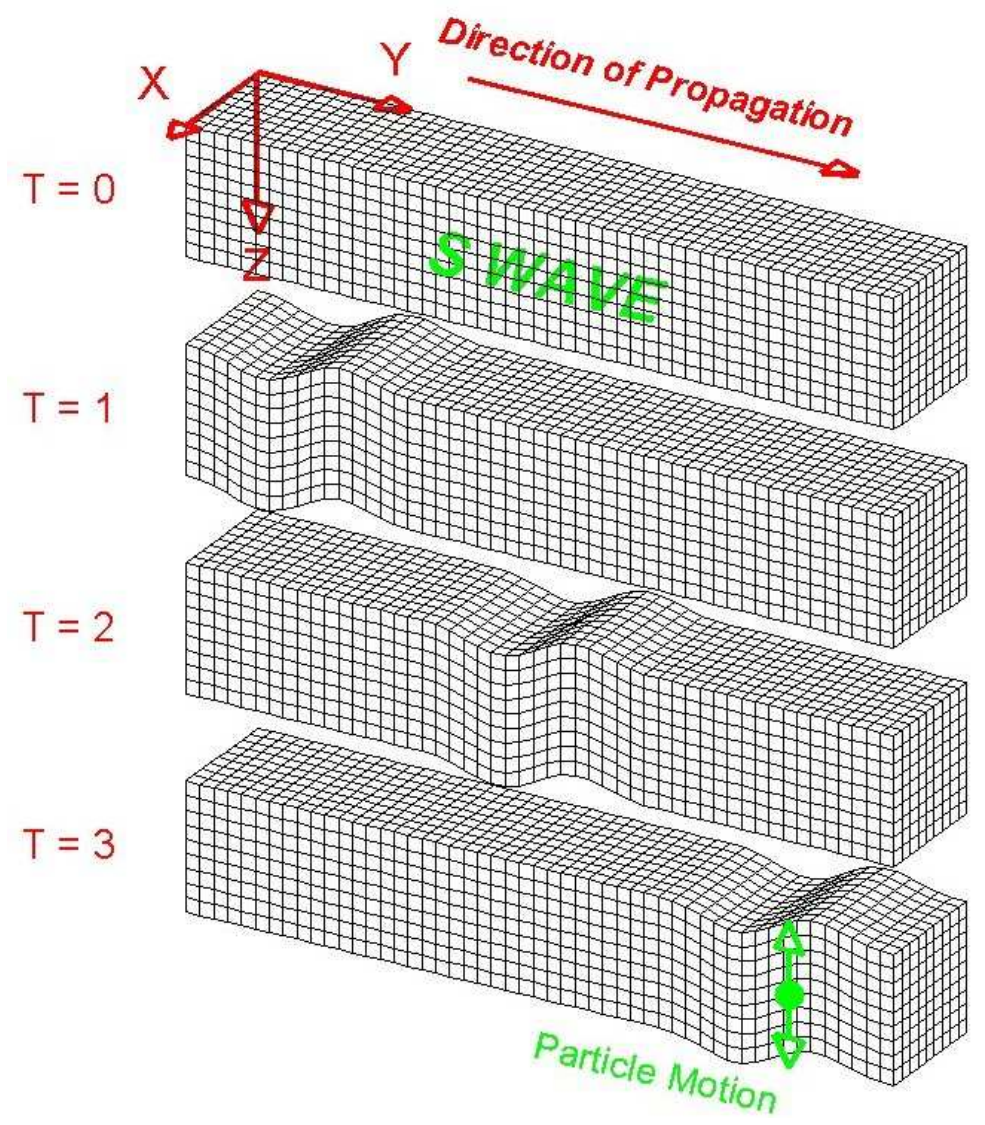

Figure 2.2 - Grid diagram showing the deformations of a solid caused by a propagating shear wave at four instances of time [18].

SV waves are more common as they can be mode converted at interfaces from other bulk waves, while SH waves are only excited using an $\mathrm{SH}$ source. A thorough treatise on $\mathrm{SH}$ waves can be found in the literature [19] [20]. Figure 2.2 shows the displacements caused by a shear SV wave propagating in a solid, again represented by a grid of square elements at four instants in time. As the shear wave propagates through the solid the grid element displacements are vertical, yet the energy propagates through the solid horizontally. 


\subsubsection{Lamb Waves}

A Lamb wave, first explored by Horace Lamb [21], is a guided wave that propagates in a free boundary plate of finite dimension when the wavelength of the incident wave is comparable to or greater than the plate thickness. Rigorous mathematical treatments of Lamb waves are given in [19], [22], and [23]. These waves are of interest when considering highly anisotropic microstructured media as the material microstructure can often be approximated by the interconnection of multiple plates. Lamb waves, as shown in Figure 2.3, are the result of the interactions between multiple $\mathrm{P}$ waves (solid lines) and $\mathrm{S}$ waves (broken lines) propagating in the plate, which have been generated by numerous reflections that occur at the plate interfaces.

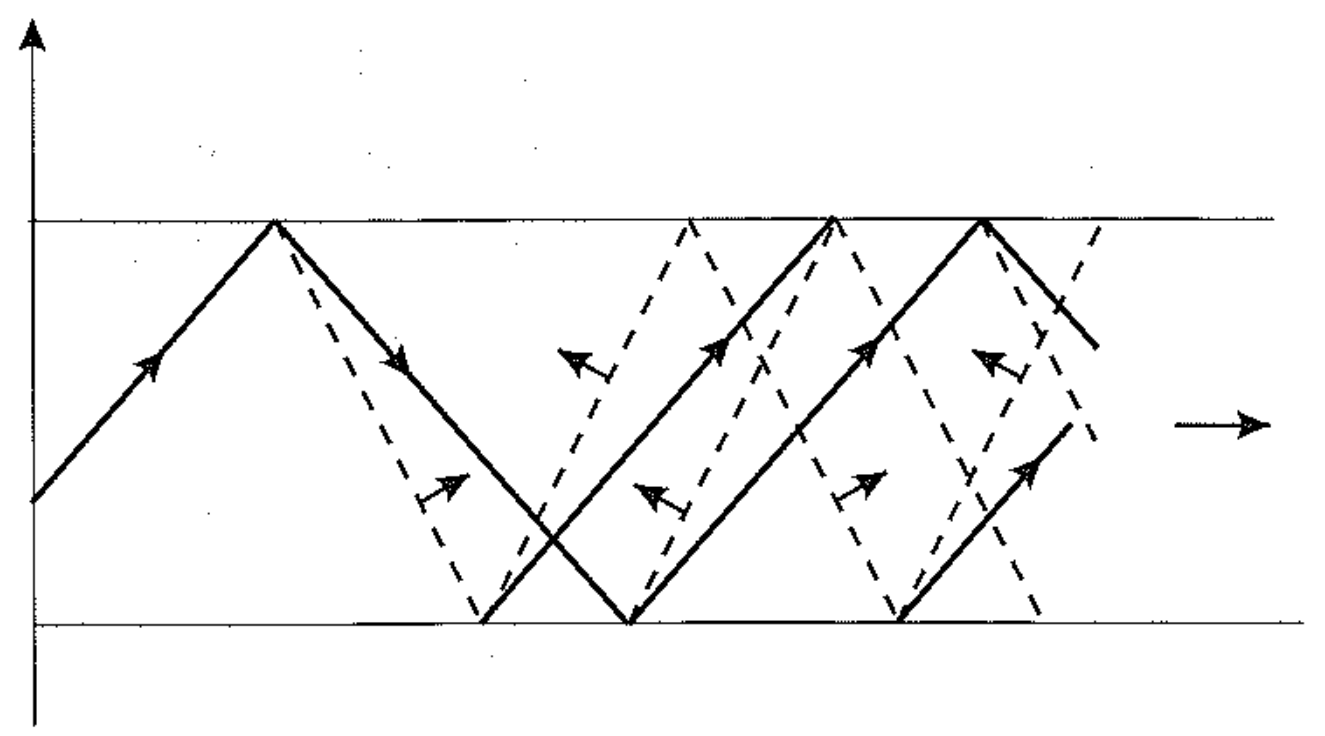

Figure 2.3 - Generation of a Lamb wave in a plate caused by the interactions of multiple P (solid lines) and S (broken lines) waves [24]. The different angles of refraction are due to the different speeds of the individual $P$ and $S$ waves.

A single $\mathrm{P}$ wave (represented by the single ray on the left) propagates at an angle through the plate. This $\mathrm{P}$ wave then reflects off the plate interface back into the plate and also generates a shear wave in the process. Now both the $\mathrm{P}$ and $\mathrm{S}$ waves generate additional $\mathrm{P}$ and $\mathrm{S}$ waves as they reflect off the other plate boundary. This process continues along the length of the plate causing the interaction of multiple $\mathrm{P}$ and $\mathrm{S}$ waves, with the superposition result of these called a Lamb wave. 
Lamb waves are highly dispersive. In other words their phase and group velocities vary with frequency. The resulting Lamb wave properties actually depend upon the frequency of excitation, the material properties, the thickness of the plate, and the angle of the incident wave with respect to the plate surface.

Lamb wave theory shows that Lamb waves can propagate as either symmetric or antisymmetric modes. These modes refer to the symmetry of the displacements about the plate axis. Considering only real solutions, the equations determining the solutions for Lamb waves in a free plate are given by equations (2.1) and (2.2) [19].

$$
\begin{aligned}
\frac{\tan (q h)}{q \tan (q h)}+\frac{4 k^{2} p \tan (p h)}{\left(q^{2}-k^{2}\right)^{2}} & =0 \quad \text { for symmetric modes } \\
q \tan (q h)+\frac{\left(q^{2}-k^{2}\right)^{2} \tan (p h)}{4 k^{2} p} & =0 \quad \text { for antisymmetric modes }
\end{aligned}
$$

where $p$ and $q$ are given by

$$
\begin{aligned}
& p^{2}=\left(\frac{\omega}{v_{L}}\right)^{2}-k^{2} \\
& q^{2}=\left(\frac{\omega}{v_{T}}\right)^{2}-k^{2}
\end{aligned}
$$

and $v_{\mathrm{L}}$ and $v_{\mathrm{T}}$ are the longitudinal and shear wave velocities respectively, $k$ is the wavenumber, $\omega$ is the angular frequency, and $h$ is half of the plate thickness. These equations can be used to plot Lamb wave dispersion curves that show the variations in phase velocity, $v_{\mathrm{p}}$, with the product of frequency $f$ and plate thickness $d$. Numerical techniques such as the bisection method [25] can be used to solve the equations. 


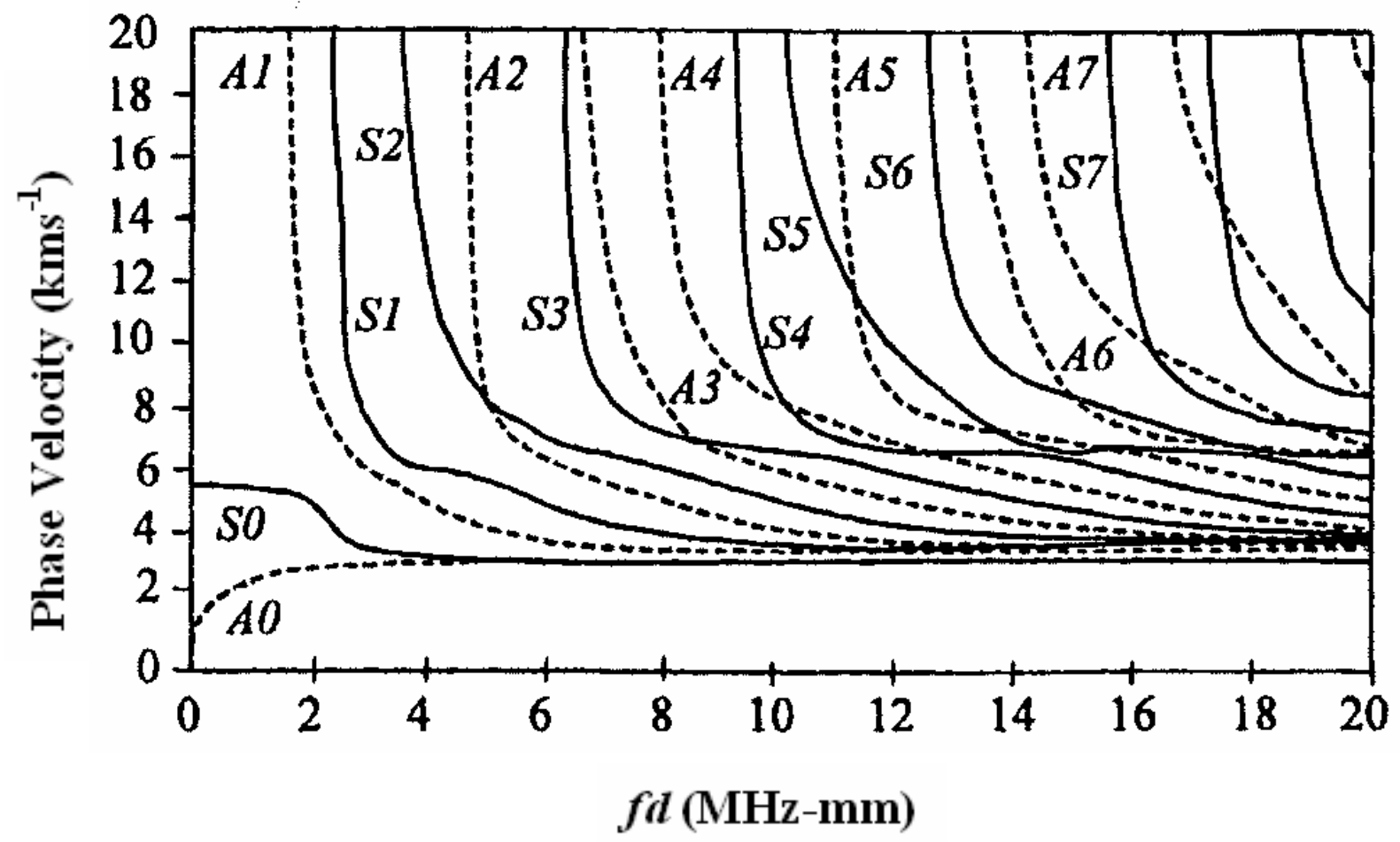

Figure 2.4 - Lamb wave phase velocity dispersion curves for a free boundary aluminium plate for both symmetric modes (solid lines) and antisymmetric modes (broken lines) [19].

The dispersion curves for an aluminium plate are plotted in Figure 2.4, showing the variations in symmetric (S) and antisymmetric (A) velocity curves from the fundamental to the seventh higher order modes with variations in $f d$. Aluminium is a suitable example material since it possesses bulk acoustic properties similar to that of amorphous alumina, the material used in later chapters that possesses a highly collinear structure (see Chapters 4,5 , and 6). It can be seen that the fundamental modes behave differently to the higher order modes with the higher order mode phase velocities asymptotically approaching infinity at certain $f d$ products. The $S_{0}$ and $A_{0}$ modes on the other hand exist all the way down to zero frequency. It can also be seen the antisymmetric mode phase velocity tends to zero whilst the symmetric mode velocity approaches a velocity between the $\mathrm{P}$ and $\mathrm{S}$ wave speeds. In the limit as $f d$ approaches zero, the $S_{0}$ mode velocity is given by (2.5) [24]

$$
v_{S 0}=2 v_{S} \sqrt{1-\frac{v_{S}^{2}}{v_{L}^{2}}}
$$


where $v_{\mathrm{S}}$ is the shear wave velocity, $v_{\mathrm{P}}$ is the longitudinal wave velocity, and $v_{\mathrm{S} 0}$ the phase velocity of the Lamb wave. It should also be noted that as $f d$ increases towards infinity, the fundamental symmetric and antisymmetric curves tend towards a common phase velocity $v_{\mathrm{r}}-$ known as the Rayleigh velocity. In this limit, the finite dimension of the plate no longer exists and an infinite media is present resulting in a Rayleigh wave propagating along the surface (see section 2.1.5). The higher order modes at infinite $f d$ converge to either the $\mathrm{P}$ or $\mathrm{S}$ bulk wave velocities of the plate media.

For a pulse (consisting of many frequencies) propagating in a plate, the individual frequencies travel at their individual phase velocities. However, the superposition of these waves, in other words the pulse envelope, travels at the group velocity $v_{\mathrm{g}}$. The group velocity $v_{\mathrm{g}}$ expressed in terms of the phase velocity is given by (2.6).

$$
v_{g}=\frac{v_{p}^{2}}{\left(v_{p}-(f d) \frac{d v_{p}}{d(f d)}\right)}
$$

Using this result the group velocity dispersion curves can be plotted using numerical differentiation. Figure 2.5 shows the resulting group velocity curves for a free boundary aluminium plate.

When the derivative of $v_{\mathrm{p}}$ with respect to $f d$ becomes zero, the phase velocity is equal to the group velocity. This can be observed by comparing Figure 2.4 with Figure 2.5. For example the $S_{0}$ mode phase velocity is constant with a derivative of zero as frequency approaches zero, hence the group velocity equals the phase velocity. As the derivative approaches infinity the group velocity tends to zero. This is observed at the cut-off frequencies of the higher order modes where with decreasing frequency the phase velocities tend to infinity and the group velocities tent to zero. Comparison of the fundamental symmetric and antisymmetric mode plate displacements reveals significantly different behaviour as shown in Figure 2.6 for an $f d$ product of 0.03 . 


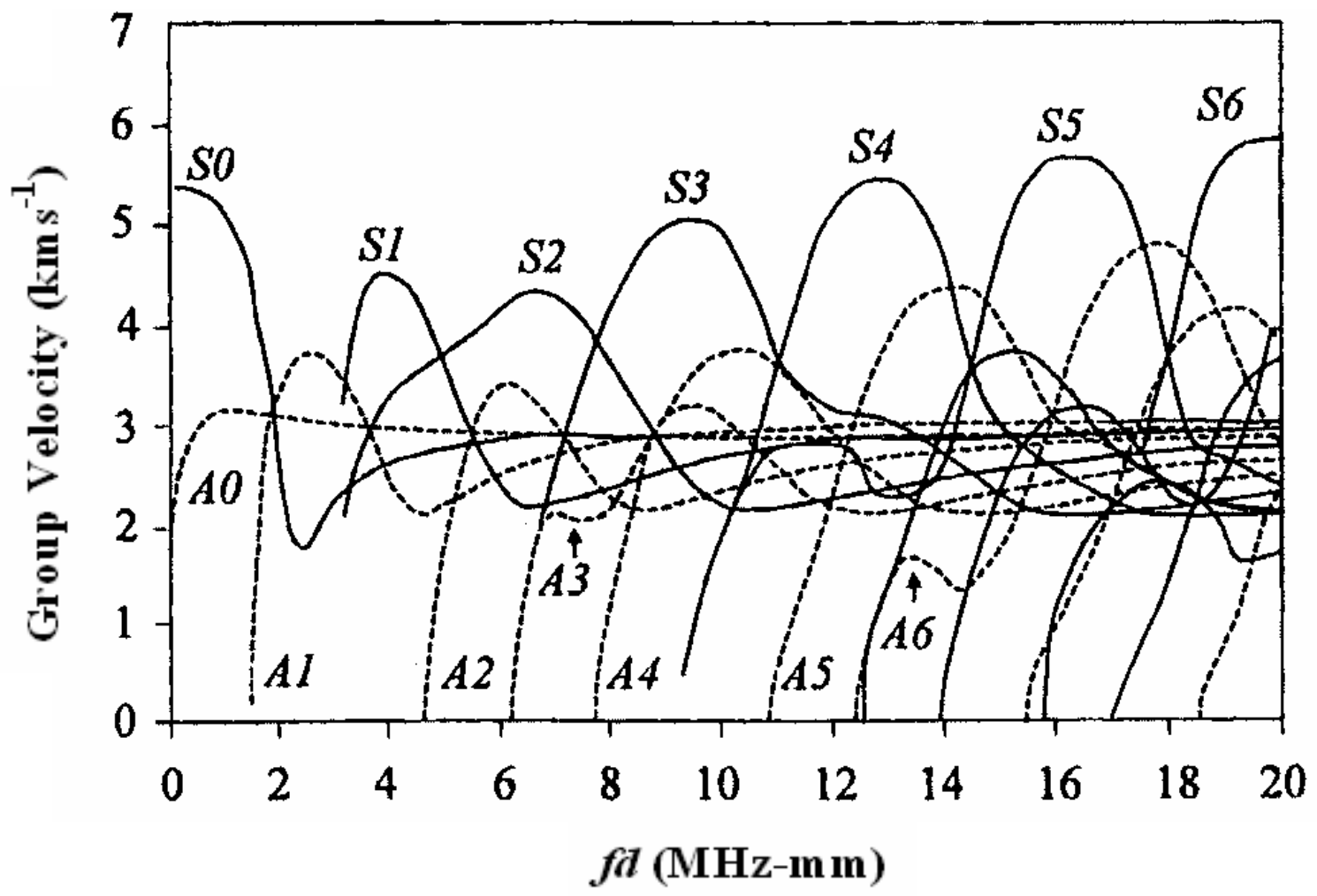

Figure 2.5 - Lamb wave group velocity dispersion curves for a free boundary aluminium plate for both symmetric modes (solid lines) and antisymmetric modes (broken lines) [19].

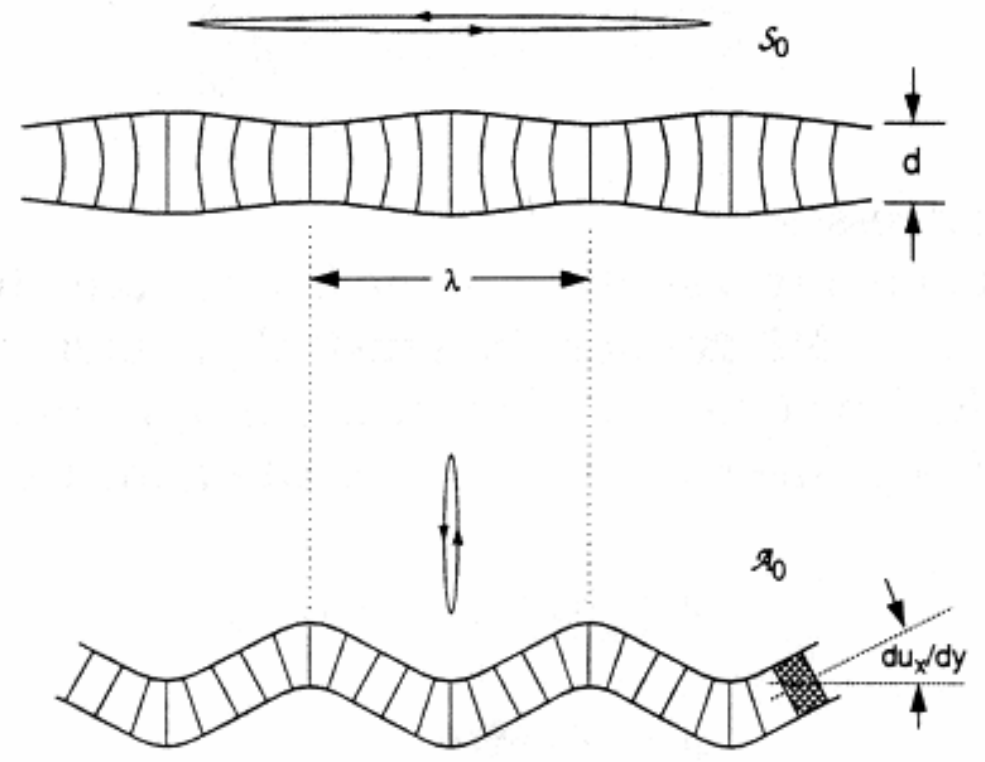

Figure 2.6 - Plate displacement shown by the circular arrows for $S_{0}$ (top) and $A_{0}$ (bottom) Lamb modes when $f d=0.03$ [24]. 
The top picture in Figure 2.6 shows the displacements caused by an $S_{0}$ mode. As can be observed this mode is dominated by longitudinal displacements with very little shear component. Hence the velocity of this mode is close to the $\mathrm{P}$ wave speed of the plate material. The antisymmetric mode shown in the lower half of Figure 2.6 predominantly consists of large transverse displacements with only small longitudinal displacements, the result of which is a slow propagating wave.

If the plate is fluid loaded, for example by water on both sides, changes in the dispersion characteristics are observed. Osborne and Hart [26] cover the theory of this case in their classic paper and then went on to confirm this experimentally in [27]. They found the phase velocity curves of the fundamental modes are little affected by the presence of the water loading, and do not change from the free boundary aluminium plate phase velocities. The effect though for the symmetric mode is the introduction of a small imaginary component to the phase velocity. This imaginary component causes attenuation of the mode due to the radiation of energy into the water, known as leaky Lamb waves. This attenuation is very low though since the $S_{0}$ mode contains displacements mainly parallel to the plate surface. The antisymmetric mode on the other hand, containing large transverse components, results in significant energy losses into the water. In addition, the presence of the water introduces two further modes not observed in the free boundary aluminium plate, one symmetric and one antisymmetric. The phase velocities of these two are almost identical to that of the water except for at low frequencies where the antisymmetric mode approaches zero, see [26] for further detail. For the situation involving different fluid loading on either side of a plate see [28].

\subsubsection{Rod Waves}

Waves in rods or bars are another form of guided wave where the propagating wave is constrained on all sides by finite dimensions relative to or less than the incident wavelength. Wave propagation in rods and bars and the calculation of the phase velocity dispersion curves is extensively covered in many references [19], [29], [30]. Not only are longitudinal and flexural modes excited in a bar, but also torsional modes. The following provides a description of longitudinal modes in a rod of radius $a$. The expression used to find the longitudinal mode phase velocity roots is given by (2.7), known as the Pochhammer frequency equation [19] 


$$
\frac{2 \alpha}{a}\left(\beta^{2}+k^{2}\right) J_{1}(\alpha a) J_{1}(\beta a)-\left(\beta^{2}-k^{2}\right)^{2} J_{0}(\alpha a) J_{1}(\beta a)-4 k^{2} \alpha \beta J_{1}(\alpha a) J_{0}(\beta a)=0
$$

where $\alpha$ and $\beta$ are given by

$$
\begin{aligned}
& \alpha^{2}=\left(\frac{\omega}{v_{L}}\right)^{2}-k^{2} \\
& \beta^{2}=\left(\frac{\omega}{v_{L}}\right)^{2}-k^{2}
\end{aligned}
$$

and $J_{0}$ and $J_{1}$ order zero and order one Bessel functions. The longitudinal phase and group velocity curves for an aluminium rod of diameter $d=2 a$ are shown in Figure 2.7 and Figure 2.8.

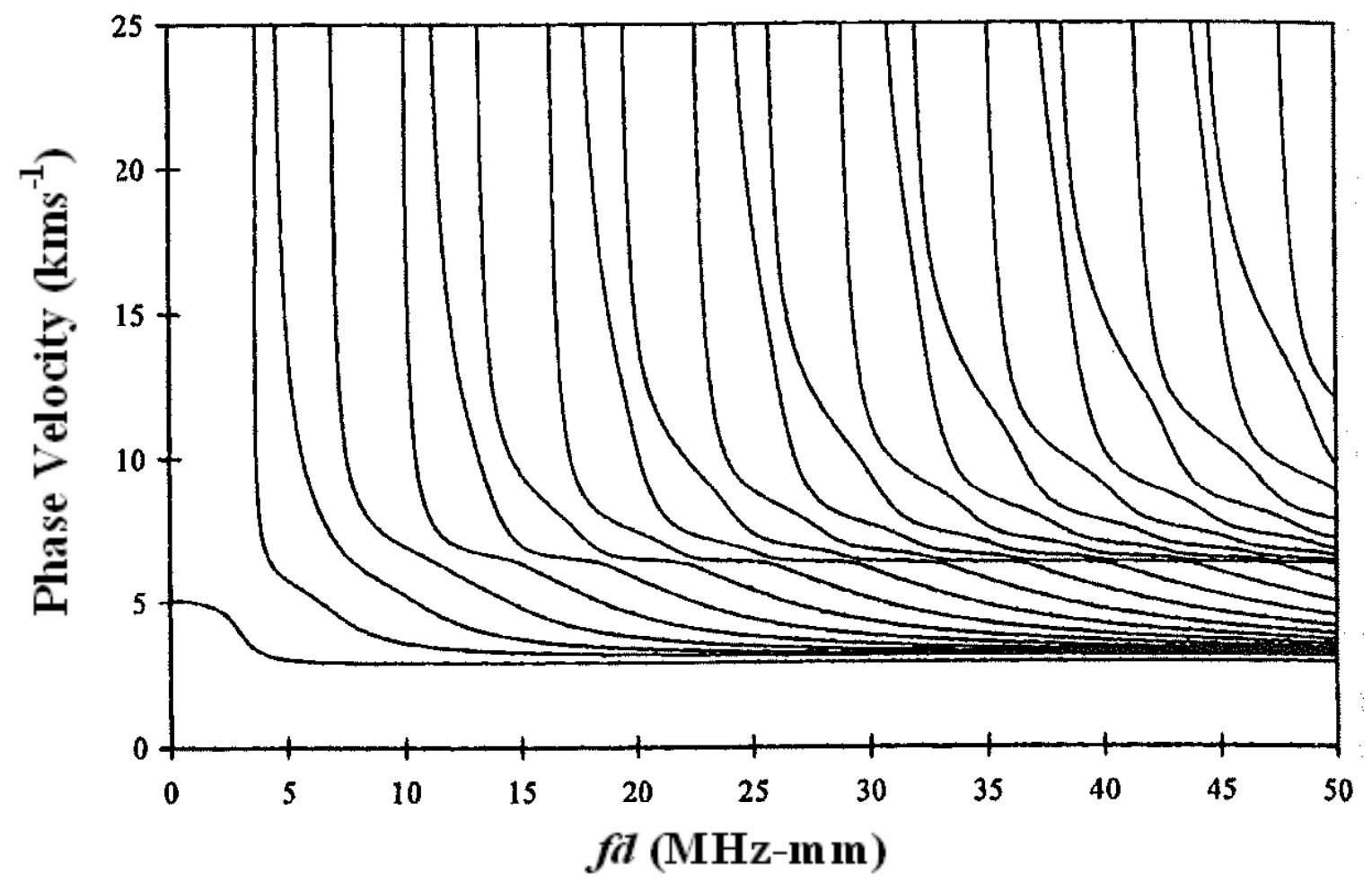

Figure 2.7 - Longitudinal mode phase velocity dispersion curves for a free boundary aluminium $\operatorname{rod}[19]$. 


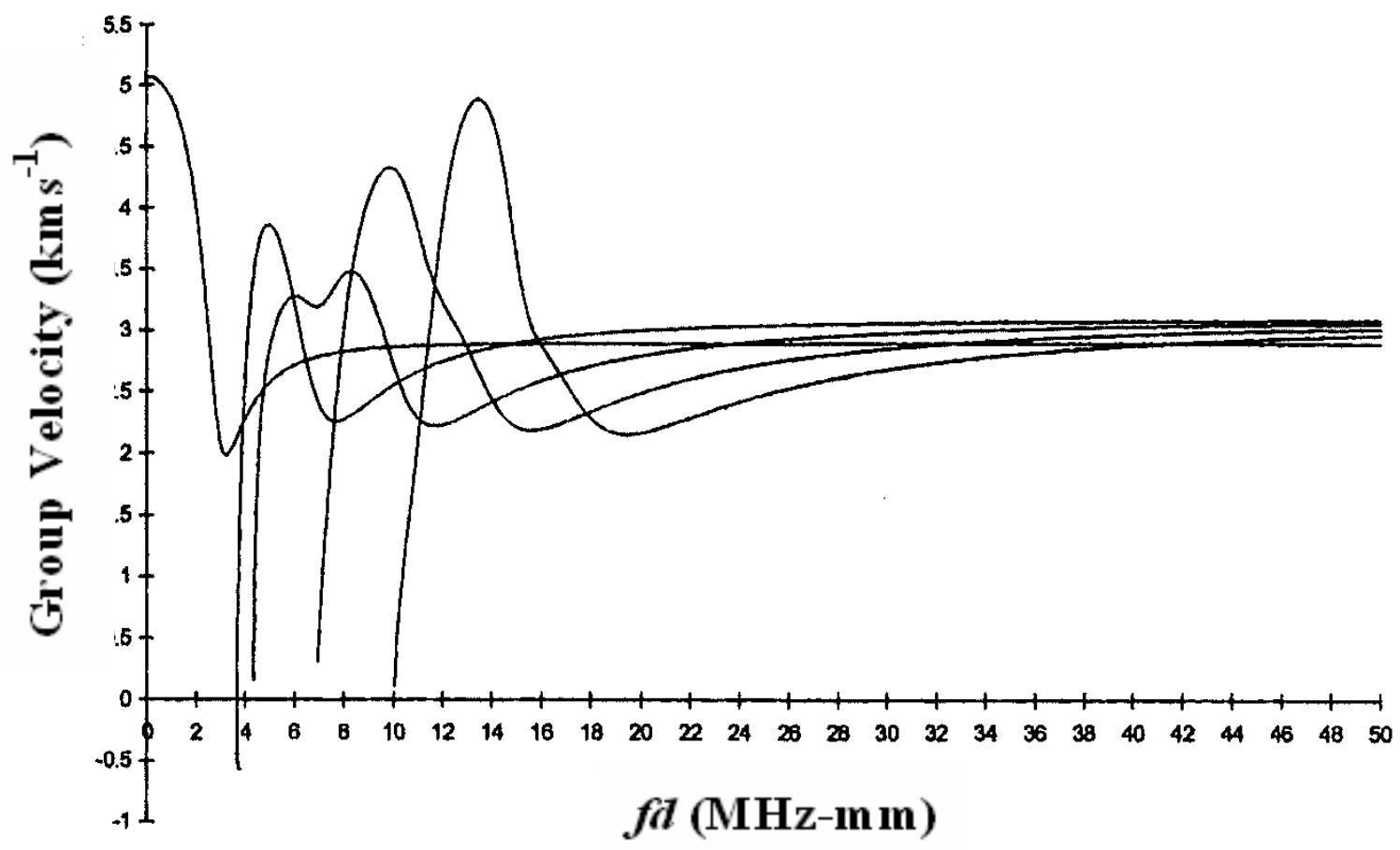

Figure 2.8 - Longitudinal mode group velocity dispersion curves for a free boundary aluminium $\operatorname{rod}[19]$.

The rod dispersion curves are very similar to those observed for the symmetric Lamb waves. In the limit as the $f d$ product approaches zero the wavelength becomes large relative to the rod diameter, and the velocity tends to a value known as the traditional bar wave velocity given by (2.10)

$$
v_{0}=\sqrt{\frac{E}{\rho}}
$$

where $E$ is the Young's Modulus of the rod material, $\rho$ is the density of the material, and $v_{0}$ is the fundamental bar velocity. This bar velocity applies for rods of any cross sectional area provided the long wavelength condition is satisfied. As $f d$ approaches infinity the fundamental mode approaches the Rayleigh velocity, whilst the higher order modes approach the $\mathrm{P}$ and $\mathrm{S}$ wave velocities of the bar material. 


\subsubsection{Rayleigh \& Interface Waves}

Rayleigh waves, first proposed by Lord Rayleigh in 1885 [31], are waves that propagate along the free surface of a solid, confined to within a wavelength or two from the surface. Due to their early discovery there are many notable references discussing Rayleigh wave propagation [23], [32]. The shear and longitudinal components of Rayleigh waves do not propagate independently, but they are coupled together and travel at a common velocity like Lamb waves. However the main difference is Rayleigh waves exist on the surfaces of free boundary materials of infinite thickness relative to a wavelength, and therefore are unlikely to be found in this work. Where Rayleigh waves propagate on the free surface of a material, waves can also propagate along the interface of two different media. These waves are known as interface waves.

\subsection{Acoustic Impedance}

The specific acoustic impedance, $Z$, of a medium is defined as the ratio of the acoustic pressure to the corresponding particle speed in that medium [33]

$$
Z=\frac{p}{v}
$$

where $p$ is the acoustic pressure and $v$ is the particle speed. The definition of acoustic impedance is analogous to the definition of electrical impedance given by Ohm's law for an electrical system. The acoustic pressure is analogous to the applied voltage and the particle velocity analogous to the current flow. For longitudinal plane waves the acoustic impedance in a medium is defined as

$$
Z_{0}=\rho v_{L}
$$

where $Z_{0}$ is known as the characteristic impedance of the medium, $\rho$ is the density of the medium, and $v_{\mathrm{L}}$ is the longitudinal velocity. This impedance is most commonly used for characterising material properties (provided plane wave incidence occurs) and is often referred to as simply $Z$. The characteristic impedance of a medium for acoustic wave propagation is 
analogous to the characteristic impedance of an electrical transmission line. As in an electrical circuit, maximum transmission of acoustic energy from one medium to another is achieved when the characteristic impedances are matched. It also follows that at times of mismatch, a portion of the incident acoustic energy will be transmitted into the second medium, and a portion will be reflected according to the degree of mismatch between media. This is discussed in the following section.

\subsection{Transmission and Reflection in Homogeneous Media}

When an ultrasonic wave encounters an interface between two homogeneous media (either media can be a fluid or solid), reflection, refraction, transmission, and mode conversion (conversion of one form of wave energy to another) can occur. The degree to with each of these phenomena occurs depends upon the types of the media, the acoustic impedances of the media, and the angle of incidence of the incident wave. Transmission and reflection phenomena that occur at a homogenous fluid-solid interface will now be described.

\subsubsection{Fluid-Solid Interface: Normal Incidence}

Consider a wavefront propagating through a homogeneous liquid with characteristic impedance $Z_{1}$, normally incident upon a homogeneous solid with characteristic impedance $Z_{2}$, as shown in Figure 2.9. At normal incidence, no mode conversion can occur. Due to the difference in characteristic impedance between the two media, the normally incident compressional wave, $p_{\mathrm{i}}$, produces a partially reflected compressional wave $p_{\mathrm{r}}$ and partially transmitted compressional wave $p_{\mathrm{t}}$ at the interface. The pressure reflection coefficient, $R_{\mathrm{p}}$, is defined as the fraction of pressure of the incident waveform that is reflected back towards the source. This is written in terms of the characteristic impedances as

$$
R_{P}=\frac{Z_{2}-Z_{1}}{Z_{2}+Z_{1}}
$$

The pressure transmission coefficient $T_{\mathrm{p}}$ is then given by

$$
T_{P}=1-R_{p}
$$




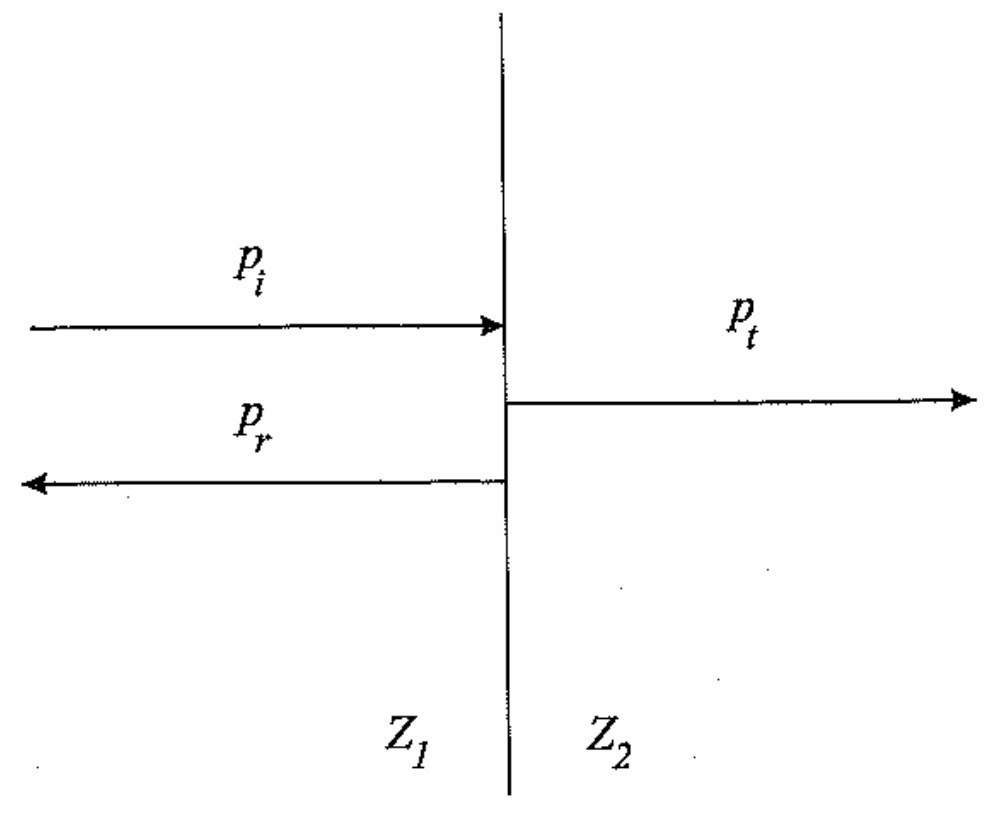

Figure 2.9 - Normally incident pressure wave reflected and transmitted at media $Z_{1} / Z_{2}$ interface.

For $Z_{2} \gg Z_{1}, R_{\mathrm{p}}=1$ and $T_{\mathrm{p}}=0$. This indicates total reflection at the interface with no energy propagating into the second medium. In the case of $Z_{2} \ll<Z_{1}$ we have $R_{\mathrm{p}}=-1$ indicating a phase change of $\pi$ occurs for all frequencies. For $Z_{1}=Z_{2}$, we have $R_{\mathrm{p}}=0$ and $T_{\mathrm{p}}=1$, which implies there is no impedance mismatch, hence maximum transmission of energy occurs when the impedances of the media are matched. The acoustic intensity is the average flux of acoustic energy per unit area per unit time and is defined in terms of the pressure and the acoustic impedance of the medium as

$$
I=\frac{p^{2}}{2 Z}
$$

Expressions for the intensity reflection coefficient $\frac{I_{r}}{I_{i}}$ and transmission coefficients $\frac{I_{t}}{I_{i}}$ are given by

$$
\frac{I_{r}}{I_{i}}=\left|R_{P}\right|^{2}
$$




$$
\frac{I_{t}}{I_{i}}=\frac{Z_{1}}{Z_{2}}\left|T_{P}\right|^{2}
$$

These equations obey the law of conservation of energy and can be verified to satisfy the requirement

$$
I_{i}=I_{r}+I_{t}
$$

\subsubsection{Fluid-Solid Interface: Oblique Incidence}

In the case of oblique incidence at a fluid-solid interface, a shear wave component can be introduced due to mode conversion. This is shown in Figure 2.10 where for an angle of incidence of $\theta_{1}$, there exists a reflected wave with angle $\theta_{\mathrm{r}}$, and two transmitted waves with refraction angles $\theta_{\mathrm{L}}$ and $\theta_{\mathrm{S}}$ corresponding to the refracted longitudinal and shear waves respectively.

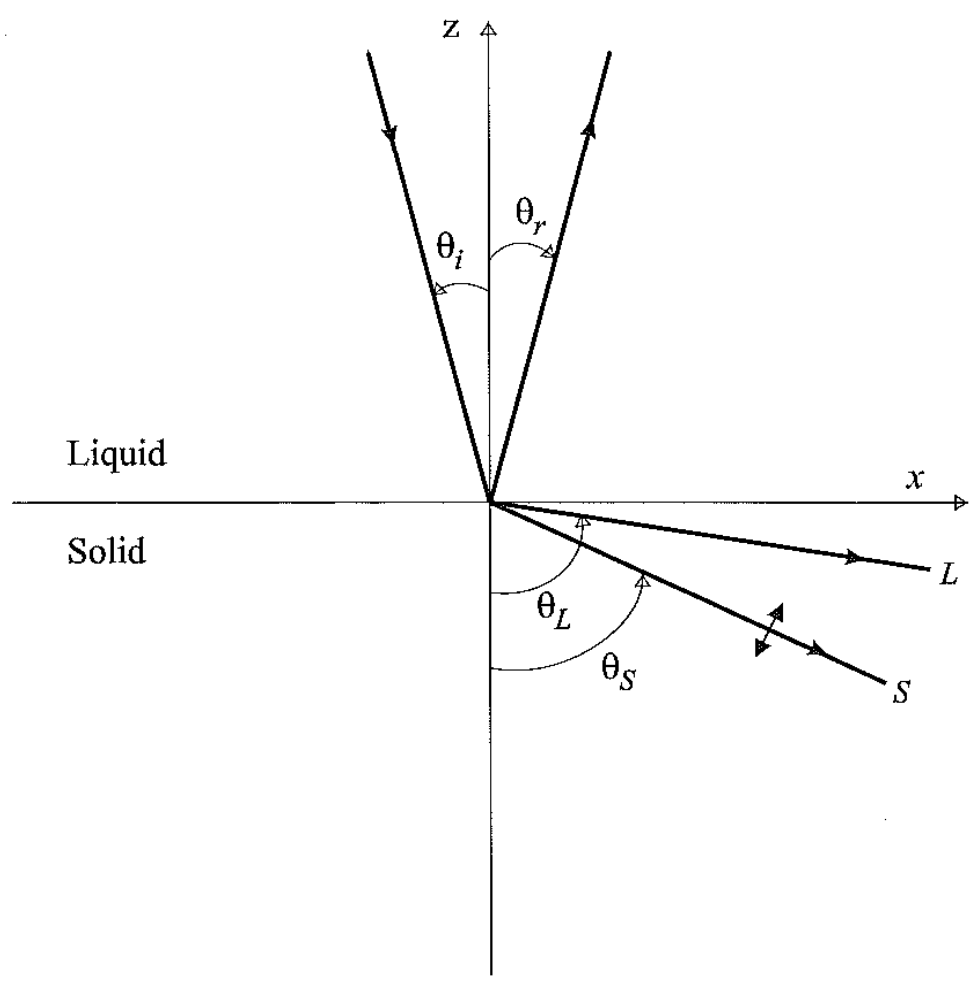

Figure 2.10 - Obliquely incident pressure wave reflected and refracted at a liquid-solid interface. 
Refraction of these waves obey Snell's law which is defined as

$$
\frac{\sin \theta_{i}}{v_{1}}=\frac{\sin \theta_{r}}{v_{1}}=\frac{\sin \theta_{L}}{v_{L}}=\frac{\sin \theta_{S}}{v_{S}}
$$

where $v_{1}$ is the velocity in the liquid, and $v_{\mathrm{L}}$ and $v_{\mathrm{S}}$ are the longitudinal and shear wave velocities of the solid respectively.

As in optics, for mechanical waves there exists angles of incidence where the refracted longitudinal and shear waves become critical, known as the first and second critical angles respectively. Beyond these angles no energy propagates into the solid. Instead propagation occurs along the interface in the form of an interface wave as defined in section 2.1.5. In the case of normal incidence, there was one reflection coefficient and one transmission coefficient. In the oblique case there is still one reflection coefficient but there are two transmission coefficients; one for the longitudinal wave and one for the shear wave. The pressure reflection coefficient for a given angle of incidence $\theta_{1}$ is given by equation (2.20) [24]

$$
R=\frac{Z_{L} \cos ^{2} 2 \theta_{S}+Z_{S} \sin ^{2} 2 \theta_{S}-Z_{1}}{Z_{L} \cos ^{2} 2 \theta_{S}+Z_{S} \sin ^{2} 2 \theta_{S}+Z_{1}}
$$

and the individual pressure transmission coefficients $T_{\mathrm{L}}$ and $T_{\mathrm{S}}$ for the transmitted longitudinal and shear waves respectively are given by [24]

$$
\begin{aligned}
& T_{L}=\frac{\rho_{1}}{\rho_{2}} \cdot \frac{2 Z_{L} \cos 2 \theta_{S}}{Z_{L} \cos ^{2} 2 \theta_{S}+Z_{S} \sin ^{2} 2 \theta_{S}+Z_{1}} \\
& T_{S}=-\frac{\rho_{1}}{\rho_{2}} \cdot \frac{2 Z_{S} \sin 2 \theta_{S}}{Z_{L} \cos ^{2} 2 \theta_{S}+Z_{S} \sin ^{2} 2 \theta_{S}+Z_{1}}
\end{aligned}
$$

where $Z_{1}, Z_{\mathrm{L}}$, and $Z_{\mathrm{S}}$ are given by 


$$
Z_{1}=\frac{\rho_{1} v_{1}}{\cos \theta_{i}} \quad Z_{L}=\frac{\rho_{2} v_{L}}{\cos \theta_{L}} \quad Z_{S}=\frac{\rho_{2} v_{S}}{\cos \theta_{S}}
$$

These equations show similarities to those for normal incidence except they are now complicated by the involvement of individual longitudinal and shear impedances. By defining an effective impedance $Z_{\text {eff }}$ [24]

$$
Z_{\text {eff }}=Z_{L} \cos ^{2} 2 \theta_{S}+Z_{S} \sin ^{2} 2 \theta_{S}
$$

the pressure reflection coefficient expression for oblique incidence can then be simplified to

$$
R=\frac{Z_{\text {eff }}-Z_{1}}{Z_{\text {eff }}+Z_{2}}
$$

which is now of the same form as the normal incidence reflection coefficient given by (2.13).

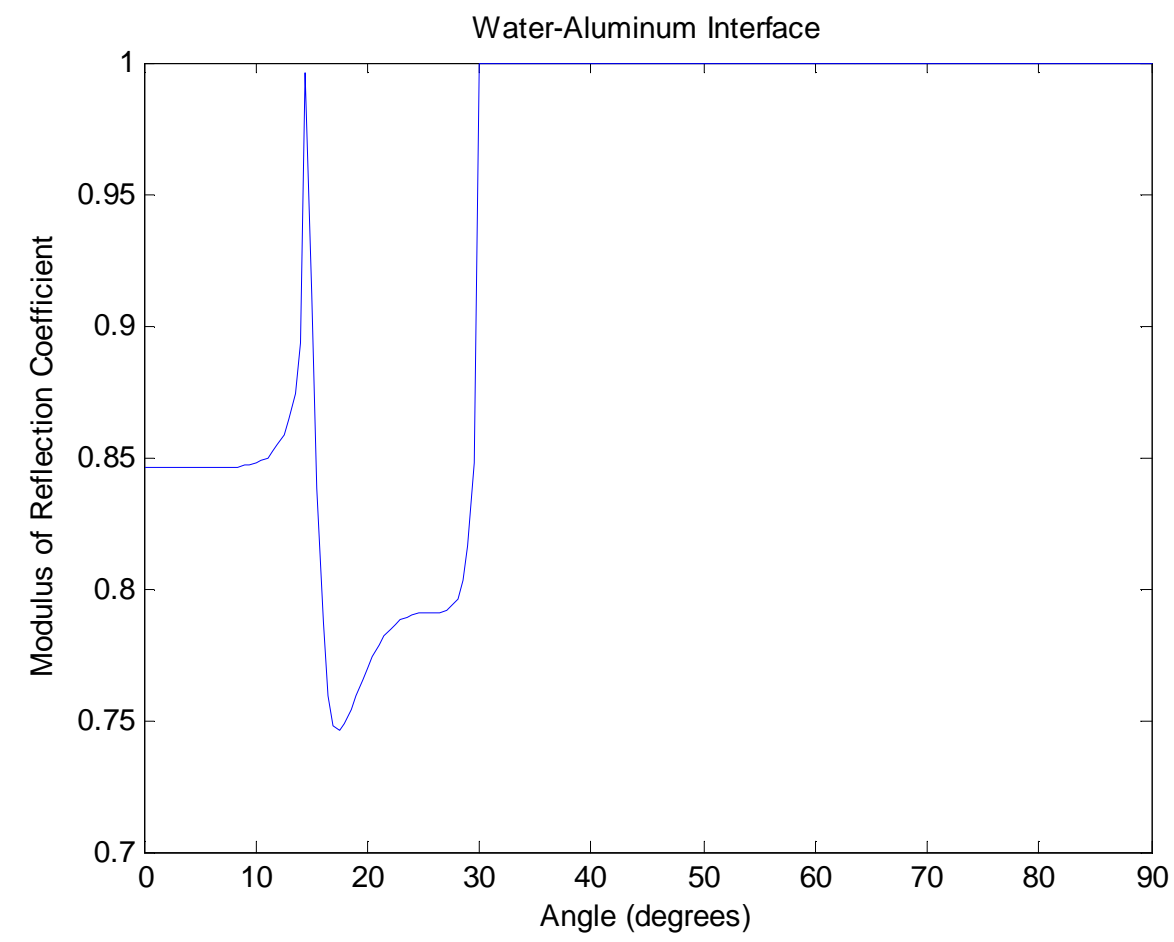

Figure 2.11 - Variation in the magnitude of the pressure reflection coefficient with angle of incidence at a water-aluminium interface. 
Using (2.20), Figure 2.11 plots the variation in the magnitude of the pressure reflection coefficient with angle of incidence for a water-aluminium interface. The properties used for water are a density of $1000 \mathrm{kgm}^{-3}$, a longitudinal velocity of $1480 \mathrm{~ms}^{-1}$, and zero shear wave velocity. For aluminium a density of $3000 \mathrm{kgm}^{-3}$ is used, a longitudinal velocity of $6300 \mathrm{~ms}^{-1}$, and a shear velocity of $3100 \mathrm{~ms}^{-1}$.

At normal incidence the reflection coefficient is solely due to the acoustic impedance mismatch between the fluid and solid, resulting in a pressure reflection coefficient of 0.85 with only a longitudinal wave transmitted. As $\theta_{1}$ increases, both longitudinal and shear waves are excited in the solid. The reflection coefficient stays relatively flat until the first critical angle of $14^{\circ}$ where the reflection coefficient rises sharply to one. At this angle the longitudinal wave becomes critical, propagating along the interface with no $\mathrm{P}$ wave energy propagating into the solid. The shear wave amplitude at this angle also goes to zero so in total no energy propagates into the aluminium. Beyond the first critical angle only the shear wave continues to propagate. When the second critical angle is reached at $29^{\circ}$, the shear wave becomes critical, also travelling along the interface of the solid with the critical $\mathrm{P}$ wave; past the second critical angle no energy propagates into the solid. Instead with both longitudinal and shear waves now critical, an interface wave propagates along the water/aluminium interface due to the interaction between the longitudinal and shear wave components.

The intensity reflection and longitudinal and shear transmission coefficients can be used to prove conservation of energy occurs. For a liquid-solid interface these are

$$
\begin{gathered}
\frac{I_{r}}{I_{i}}=|R|^{2} \\
\frac{I_{L}}{I_{i}}=\frac{\rho_{2} \tan \theta_{i}}{\rho_{1} \tan \theta_{L}}\left|T_{L}\right|^{2} \\
\frac{I_{S}}{I_{i}}=\frac{\rho_{2} \tan \theta_{i}}{\rho_{1} \tan \theta_{S}}\left|T_{S}\right|^{2}
\end{gathered}
$$


where $\frac{I_{r}}{I_{i}}$ is the energy reflection coefficient, $\frac{I_{L}}{I_{i}}$ the longitudinal wave transmission coefficient, and $\frac{I_{S}}{I_{i}}$ the shear wave transmission coefficient. The percentage of energy reflected, transmitted as a longitudinal wave, and transmitted as a shear wave for a wateraluminium interface is plotted in Figure 2.12.

For non-normal angles of incidence, before the first critical angle, the longitudinal wave possesses more energy than the shear wave, but this difference becomes less as the angle of incidence approaches the first critical angle. Between the first and second critical angles, only the shear wave propagates with more energy than the longitudinal wave possessed. The energy reflected is also reduced in this region. For angles beyond the second critical angle no energy propagates into the solid; conservation of energy is preserved for all angles of incidence.

What Figure 2.11 and Figure 2.12 do not distinguish is for angles of incidence greater than either of the critical angles, what fraction of the reflected energy is travelling along the interface of the solid and what fraction is reflected into the fluid.

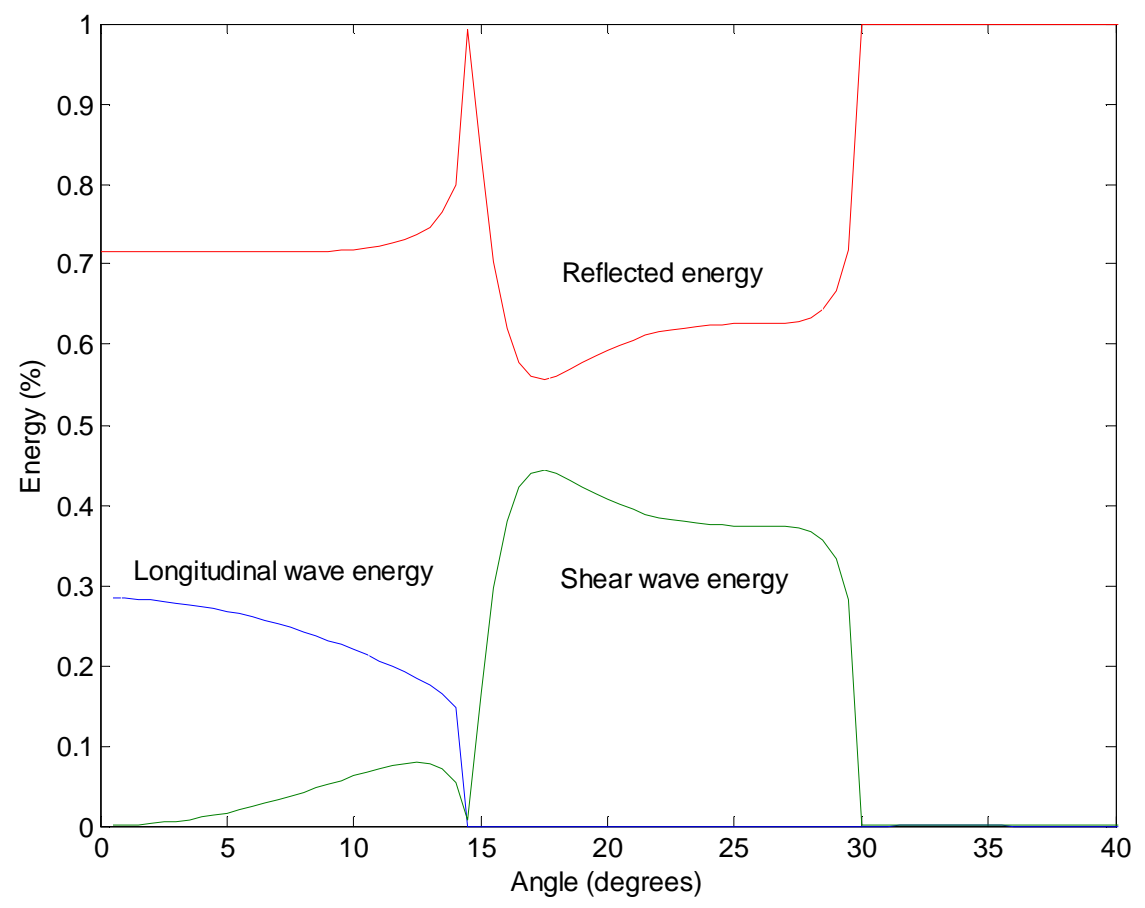

Figure 2.12 - Percentage energy reflected and transmitted as shear or longitudinal waves at a water-aluminium interface. 


\subsection{3 $\lambda / 4$ Wavelength Matching Layers}

As described previously, differences in acoustic impedance between two media generates reflected waves from the media interface, reducing the energy transmitted into the second medium. The larger the impedance mismatch the greater the amount of energy reflected from the interface. This is a significant problem in medical ultrasound due to the large acoustic mismatch between the piezoceramic (piezoelectric material of a transducer), 30 MRayls, and the very low impedance of the biological tissues to be examined, 1.5 MRayls.

To reduce the impedance mismatch between the two media, a common method is to perform impedance matching to increase the energy coupled from the first media into the second. Impedance matching is the insertion of an intermediate layer that is placed in between the first and second media [33]. The acoustic impedance of the matching layer for maximum energy transfer between the two media is given by

$$
Z_{\text {match }}=\sqrt{Z_{1} Z_{2}}
$$

where $Z_{\text {match }}$ is the acoustic impedance of the introduced matching layer, $Z_{1}$ is the acoustic impedance of the first media, and $Z_{2}$ is the acoustic impedance of the second media. The impedance of the matching layer is thus the geometric mean of the first and second layer impedances. However, the process is selective in that total transmission of energy only occurs at a single frequency. This is only achieved if the matching layer thickness $d$ is made to a quarter of the wavelength in the layer.

$$
d=\frac{\lambda}{4}
$$

Using this thickness ensures waves that were reflected within the matching layer are in phase when they exit the layer. Almost maximum energy transmission will occur for a narrow band of frequencies around this single frequency, significantly improving the energy transmission. This method is similar to the quarter wavelength sections used to match an antenna to an electrical transmission line. 
Transducers with single matching layers have been shown in [34] to possess fractional bandwidths (bandwidths specified as a fraction of the central transducer frequency) of between 40-50\%. This can be improved upon as shown in [35]-[37] using two matching layers, improving the fractional bandwidth to around $70 \%$. In [38], [39] transducers with many matching layers are considered with further bandwidth improvement. From these works, for medical transducers, due to the large impedance mismatch between tissues and the transducer piezoceramic, matching impedances in the range of 3-14 MRayl are often required, with 7-12 MRayl the most common requirement. Unfortunately very few single-phase solids exist with these useful impedances. Table 2.1 shows the acoustic properties of some common metals [24], plastics [24], biological tissues [40], and piezoceramics [24].

Table 2.1 - Acoustic properties of common metals, plastics, biological tissues, and piezoceramics.

\begin{tabular}{lllll}
\hline Material & $\begin{array}{l}\text { Density } \\
\left(\mathrm{kgm}^{-3}\right)\end{array}$ & $\begin{array}{l}\text { Longitudinal Velocity } \\
\left(\mathrm{ms}^{-1}\right)\end{array}$ & $\begin{array}{l}\text { Shear Velocity } \\
\left(\mathrm{ms}^{-1}\right)\end{array}$ & $\begin{array}{l}\text { Acoustic Impedance } \\
(\text { MRayl })\end{array}$ \\
\hline Metals & & & 3100 & 17.0 \\
\hline Aluminium & 2700 & 6300 & 700 & 24.6 \\
Lead & 11200 & 2200 & 3200 & 46.4 \\
Iron & 7690 & 5900 & 1380 & 3.27 \\
\hline Plastics & & & 1150 & 2.52 \\
\hline PVC & 3270 & 2380 & 540 & 1.76 \\
Polystyrene & 1050 & 2400 & & 1.647 \\
Polyethylene & 900 & 1950 & & 1.645 \\
\hline Tissues & & & & 34.657 \\
\hline Heart & 1060 & 1554 & & 34.3 \\
Muscle & 1041 & 1580 & & \\
Liver & 1050 & 1578 & & \\
\hline Piezoceramics & & 4560 & & \\
\hline PZT-5A & 7750 & 4350 & & \\
PZT-5H & 7500 & 4560 & & \\
\hline
\end{tabular}




\subsection{Wave Propagation in Anisotropic Media}

The previous section described the fundamental transmission and reflection phenomena for plane wave propagation in bulk homogeneous and isotropic media. For waves propagating in anisotropic media the properties of the waves are directionally dependent, with the possibility of multiple modes propagating. As a result the solutions for anisotropic media are more complicated and the same theory may not apply. Ultrasonic waves propagating in anisotropic media have been studied for decades in the broad areas of geophysics, non-destructive testing, and medical ultrasound, with materials varying from tissues to highly structured fibre reinforced composites. This section provides an indication of the influence that anisotropic microstructure has on ultrasound, and a summary of relevant previous studies is presented. Emphasis is placed upon those involving high frequency ultrasonic waves and collinear solids and fibrous tissues.

\subsubsection{Fibrous Reinforced Layers}

Unlike most homogeneous and isotropic media where bulk waves propagate with the same velocity and displacement properties independent of frequency (can have homogeneous dispersive media such as sea water), the structure and dimensions of microstructured media determine the properties of the propagating waves leading to dispersive solutions. An example from [19] demonstrates this by varying the fibre orientation in an arbitrary ideal unidirectional fibre reinforced composite plate and examining the variations in velocity that occur. The regularly spaced fibres run through the entire plate with the diameter of the fibres the same as the plate thickness. The influence of the angle of incidence relative to the fibre orientation on velocity is investigated by plotting the phase velocity dispersion curves for parallel and perpendicular incidence in Figure 2.13 and Figure 2.14 respectively. The derivation of these curves can be found in [19]. As can be observed the fibre direction greatly influences the velocity with much larger values for propagation parallel to the fibre orientation.

In ultrasonic non-destructive testing considerable work has been performed on studying wave propagation in layered media, with the wave propagation in layered fibre reinforced composite plates covered extensively in the literature [41]-[46]. Figure 2.15 shows the different types of anisotropic architecture these composites can display. Reference [44] provides an excellent review of the major techniques and methods used for analysing wave propagation in these different types of composites. 


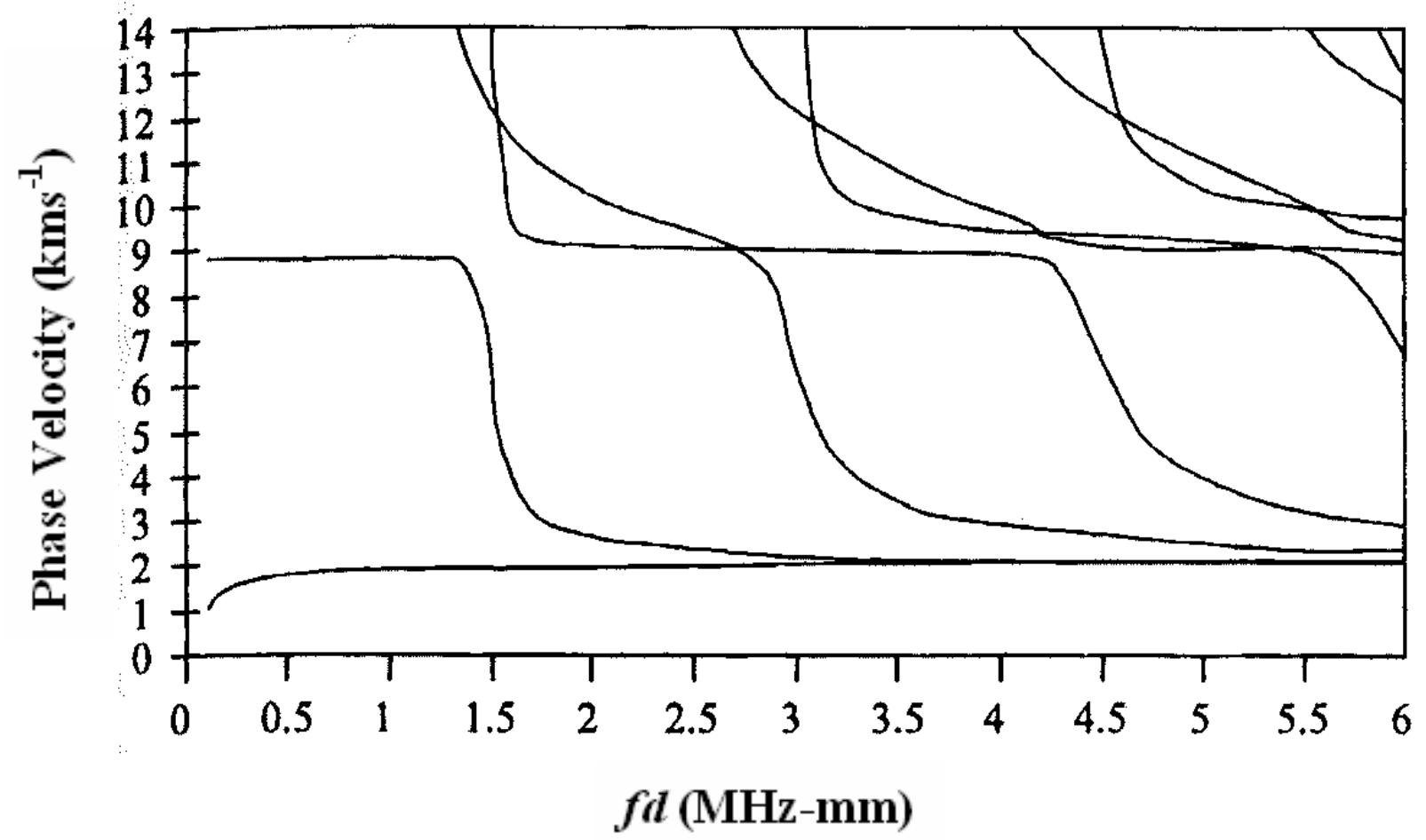

Figure 2.13 - Phase velocity dispersion curves for a unidirectional fibre reinforced composite plate for propagation parallel to the direction of fibre alignment [19].

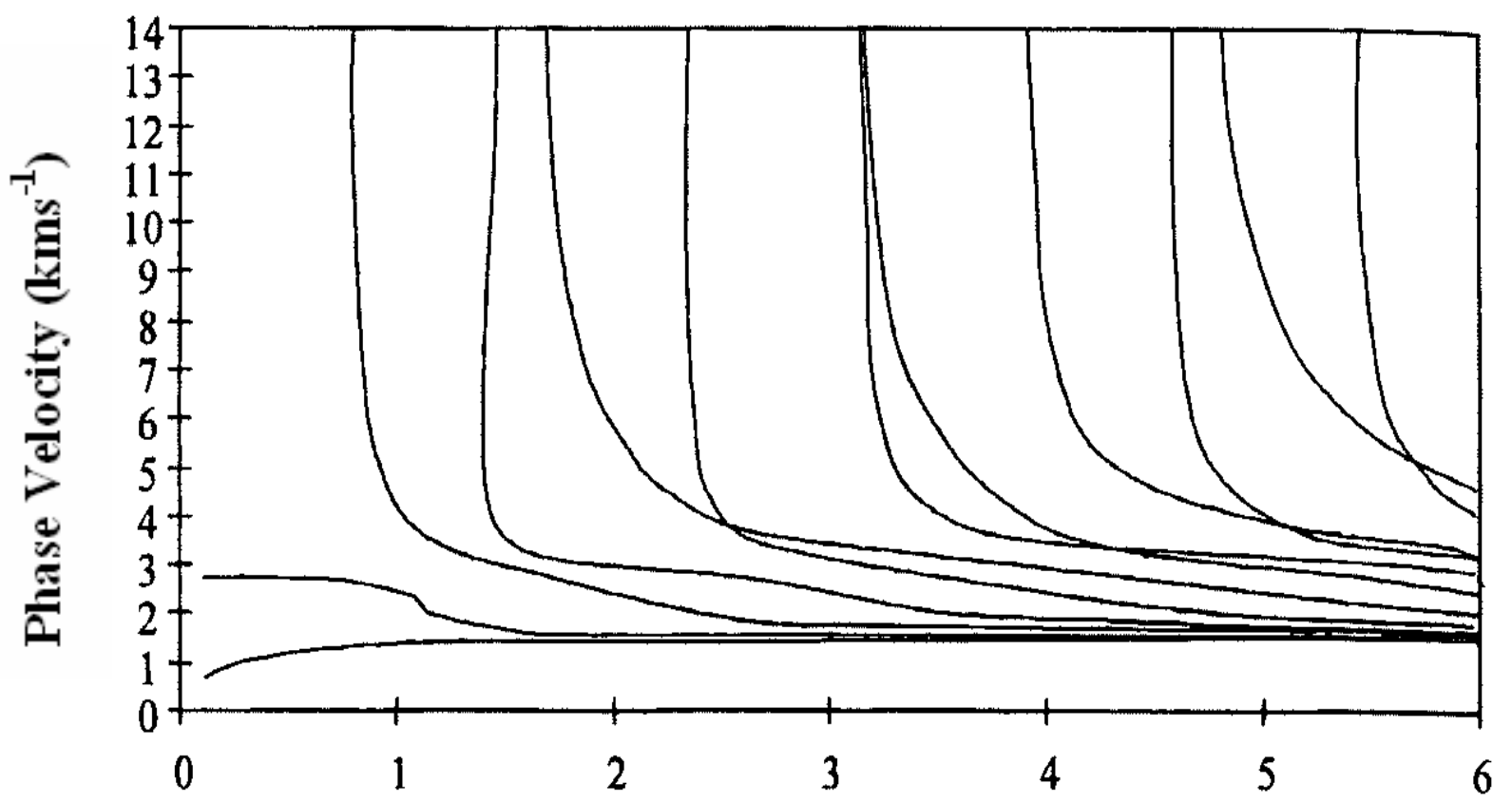

$f d(\mathrm{MHz}-\mathrm{mm})$

Figure 2.14 - Phase velocity dispersion curves for a unidirectional fibre reinforced composite plate for propagation perpendicular to the direction of fibre alignment [19]. 


\section{Fiber Orientations in Fiber}

Rein forced Composites

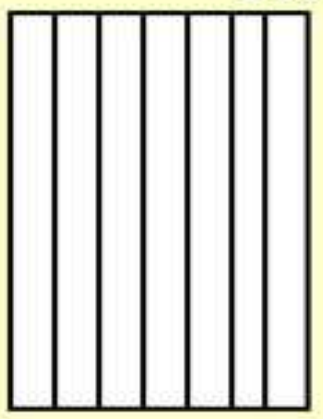

Continuous and aligned fibers

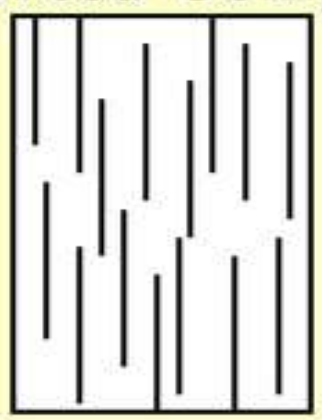

Discontinuous and aligned fibers

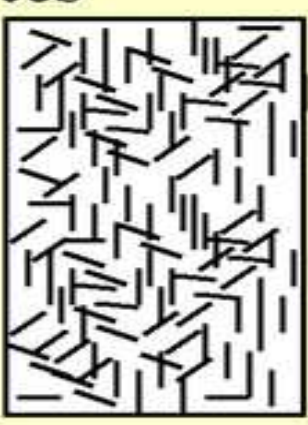

Discontinuous and randomly oriented fibers

Figure 2.15 - Examples of the different types of fibre reinforced composites.

This work was extended in [45] where an alternative method was developed to analyse a radial stacked three-layered fibre reinforced composite sample, with all three layers containing fibres parallel to the direction of orientation. The middle layer fibre orientation was then varied to analyse the influence on the velocities and modes observed. The analysis of Lamb wave propagation in axially multi-layered inhomogeneous free boundary waveguides was then analysed in [46].

\subsubsection{Collinear Porous Solids}

Highly regular collinear porous solids are of interest in this work as they represent an ideal structure to begin this study. An understanding of the wave propagation in these samples facilitates understanding of the propagation in less ideal structures. Several studies using high frequency ultrasound have been undertaken to characterise highly structured ceramics.

The characterisation of advanced porous alumina, sialon, and zirconia ceramics using 75-100 MHz ultrasonic waves was presented in [47]. By measuring the wave delay time and pulse widths after propagation through the ceramic, sample porosities, pore shapes, and pore sizes were statistically determined. Although highly anisotropic these samples were not regular in structure, demonstrating poor collinearity and random pore spacing. 


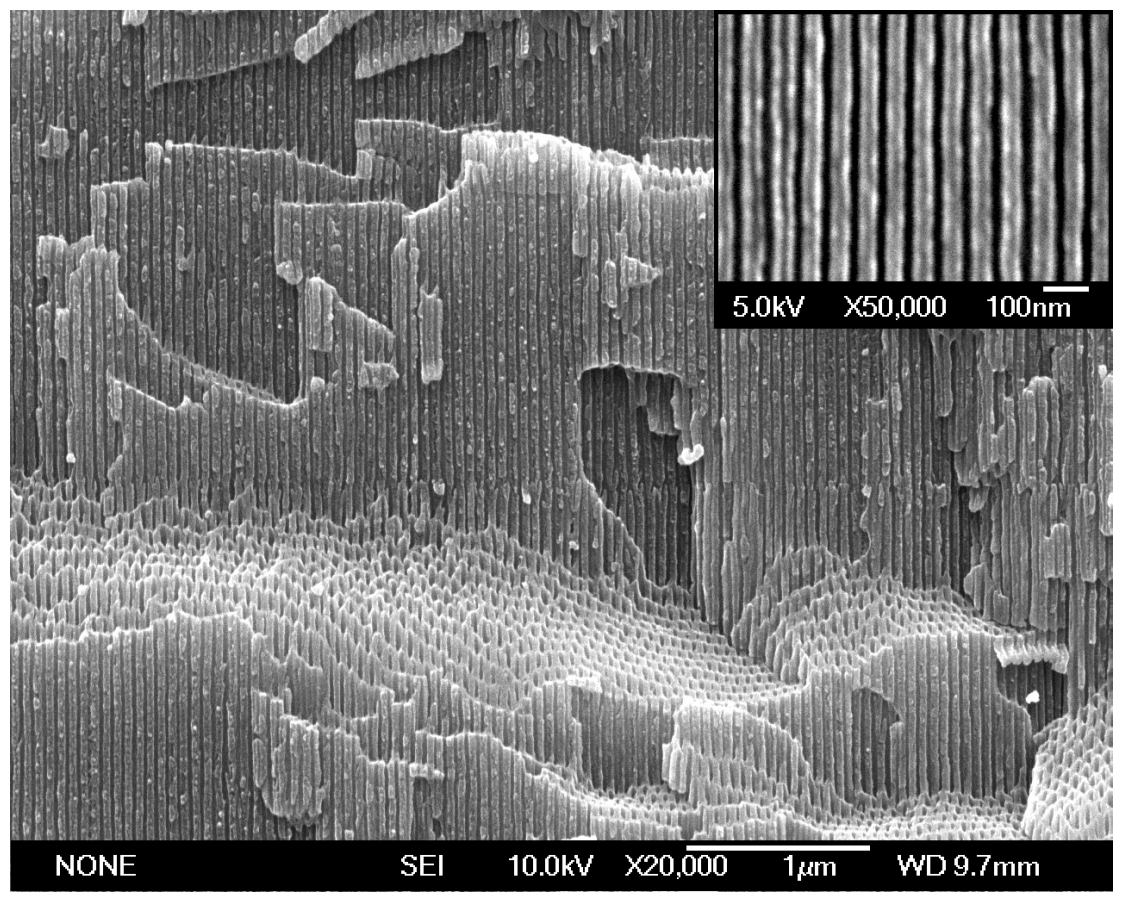

Figure 2.16 - Longitudinal cross section of a porous anodic alumina sample. The pores extend from the top of the sample to the bottom with no irregularities. The sample was physically snapped in half giving rise to the observed broken pores.

Since 1995 alumina ceramics have attracted considerable attention in numerous fields due to the periodic pore arrangements and high collinearities attainable by anodising high purity aluminium. Porous anodic alumina (PAA) samples are highly regular collinear porous ceramics, containing nanometer-sized dimensions. Figure 2.16 shows a longitudinal cross section of an example PAA sample. Here sample pores can be seen to extend from the top of the sample through to the bottom with no irregularities in between; the regularity is better observed in the inset image. The sample in this case was physically snapped in half resulting in the observed broken pores. This structure makes them an ideal template for preparing advanced nanostructures for a wide range of materials [48]. They have been used as templates to produce photonic crystals [49], micropolarizers [50], polymeric nanostructures [51], nanometals [52], and carbon nanotubes [53]. They are also commonly used in the aerospace industry as adhesion layers as reported in [54], and for filtration [55] and gas separation [56].

Significant characterisation of the bulk mechanical properties of both amorphous and crystalline PAA samples has been performed using high frequency ultrasound. In [57] quantitative acoustic microscopy was used to measure the surface wave velocities on anodised aluminium 
substrates to be used as adhesion layers, using ultrasonic wave frequencies up to $1 \mathrm{GHz}$. Here it was found the surface wave velocity showed significant dependence on the porosity of the alumina film. These velocities were found to be highly dispersive, allowing small changes in the material surface properties to be detected, providing an excellent method for the nondestructive testing of these layers.

Acoustic microscopy, using surface wave frequencies up to $225 \mathrm{MHz}$, was used in [58] to determine the density and elastic constants of anodic alumina films of 1-22 $\mu \mathrm{m}$ thickness. The elastic constants were determined by measuring the surface wave dispersion curves in the film, and the dependence of the attenuation coefficients on the PAA film thickness used to calculate the density. The influence of porosity on the Young's modulus, Poisson's ratio and velocities in non-anodic heat-treated alumina ceramics was investigated in [59]. Here sintering was used to induce a level of irregular, non-collinear porosity into the alumina. The longitudinal velocity, shear velocity, and Young's modulus were found to vary significantly with the sample porosity. The mechanical properties of anodic alumina samples were also measured in [60] and [61] using alternative nanoindentation methods.

In the literature distinction has been made between amorphous and crystalline alumina. It is revealed the bulk crystalline alumina acoustic properties (e.g. Young's modulus, shear modulus etc.) are significantly greater ( $>3$ times) than amorphous alumina structures. By annealing the amorphous samples at high temperatures $\left(>800{ }^{\circ} \mathrm{C}\right)$, the alumina structure changes from an amorphous to a crystalline structure, significantly increasing the mechanical strength. The work reported in [62] provides an excellent summary of this by analysing the variation in the elastic behaviour of partially sintered alumina bodies that have been subjected to the initial stages of sintering. Reference [63] performed structural characterisation of heat-treated porous anodic alumina at various temperatures to transform the anodic alumina from the amorphous phase to the final crystalline phase, also known as corundum. At the various temperatures the increase in sample crystallinity can be observed to develop, modifying the acoustic properties of the alumina at each stage.

Whilst very high frequency acoustic microscopy has been used to characterise thin film PAA using surface wave propagation methods, studies of the high frequency bulk acoustic transmission properties (e.g. velocity, acoustic impedance, and attenuation) in highly collinear 
PAA with variations in the structural properties are not found in the literature. The variation in these acoustic properties of anodic alumina with changes in the microstructure is of interest to determine the suitability of these samples for applications such as acoustic impedance matching. The nanometer-sized dimensions of these samples significantly influence high frequency ultrasonic (> $50 \mathrm{MHz}$ ) propagation, leading to guided wave modes. This work aims to fill this gap by analysing the ultrasonic transmission properties of PAA structures using high frequency ultrasound.

\subsubsection{Fibrous Tissues}

In medical ultrasound anisotropic tissues are frequently encountered. Various human tissues such as muscle and skin are known to consist of significantly fibrous microstructures. This can be observed in Figure 2.17 that shows the highly fibrous and anisotropic nature of a human myocardium (heart tissue) sample, displaying a preferred orientation along the layer.

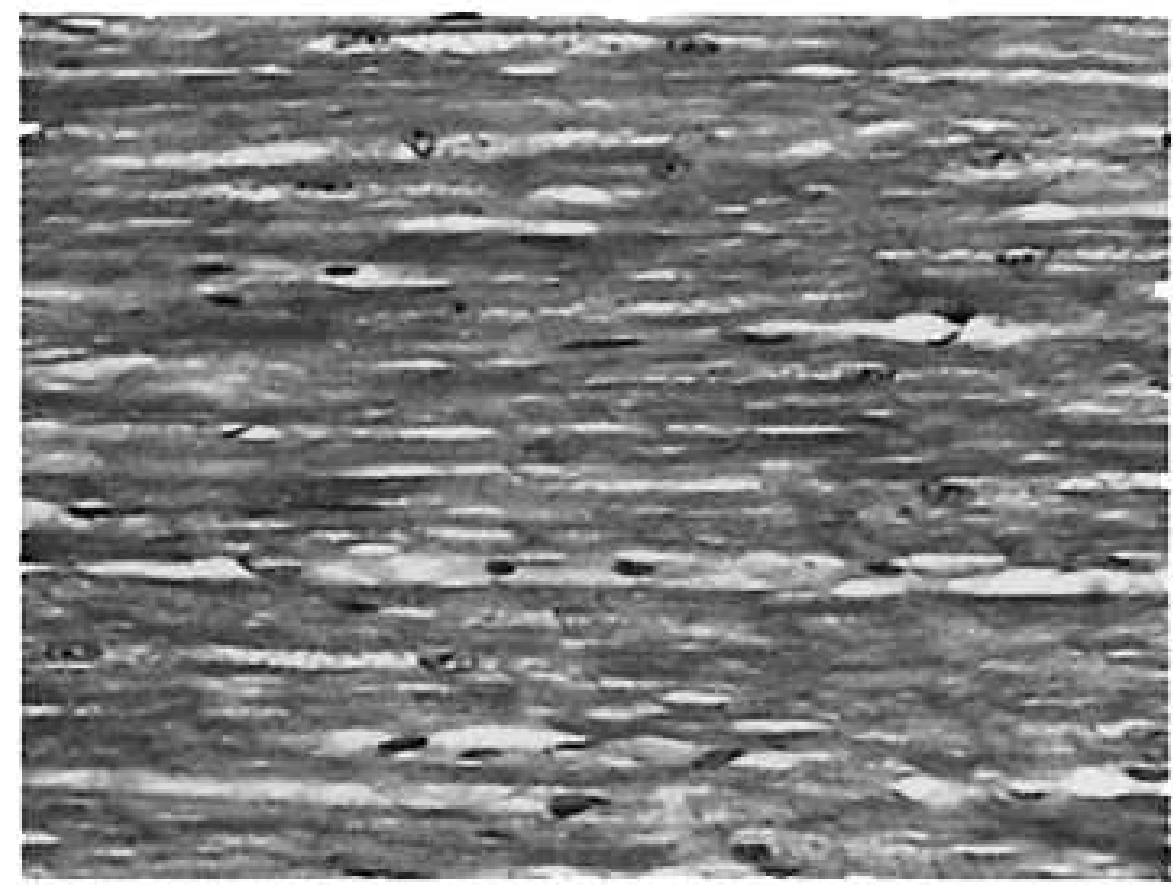

Figure 2.17 - Myocardium tissue sample from a human heart that demonstrates a fibrous anisotropic microstructure [125].

However as described in chapter 1, all of these fibrous tissues are treated as homogeneous in current diagnostic ultrasound imaging systems. In numerous articles found in the literature such as [40] and [64], the direction of tissue fibre orientation has been considered, with 
characterisation performed both parallel and perpendicular to the direction of fibre alignment. Characterisation involved measurements of both velocity and attenuation, which were found to differ depending on the fibre orientation relative to the incident illumination angle. Whilst the anisotropy of the tissues were revealed, these studies did not consider the influence of underlying fibrous microstructures, and their potential waveguiding affect on high frequency ultrasound propagation.

In [65] skin collagen microstructure was considered by analysing the ultrasonic backscatter from skin lesions at $20 \mathrm{MHz}$. Using this technique localised microstructural variations were determined by relating the variations in the measured backscatter coefficients to the differences in the distribution of collagen fibres. However, the collagen fibres were treated as scatterers in that work. Reference [66] characterised skin lesions using a $33 \mathrm{MHz}$ system to identify relationships between dermatitis and the thicknesses of the various skin layers e.g. dermis and epidermis, found in skin. This was performed using an echo-based system where the echogenicity of the skin was used to determine these relationships; again the underlying waveguiding properties of the fibrous tissues were not considered. With imaging systems continuing to increase in frequency, the waveguiding influence of fibrous tissues has an increasing role, and as such additional information is likely to be revealed otherwise unattainable using current methods.

\subsection{Attenuation}

Attenuation is the decrease in signal amplitude of an ultrasonic wave as a result of passage through some medium. This occurs through two different mechanisms - scattering and absorption. The total attenuation is equal to the sum of the individual scattering and absorption contributions.

\subsubsection{Scattering}

The scattering of acoustic waves is the redirection of energy caused by the interaction of a wave with the internal structure of a material. The degree and type of scattering depends upon the size, shape, and orientation of the scatterer relative to the incident wavelength. The type of scattering falls into three main categories: objects much larger than, objects much smaller than, and objects comparable to a wavelength. Scatterers in this section are considered to be rigid 
spheres with the product $k a$ (where $k$ is the wavenumber given by $k=\frac{2 \pi}{\lambda}$, and $a$ is the radius of the sphere) used to express the incident wavelength relative to the radius of the sphere. Only brief descriptions are given for each type of scattering since [67] provides a good summary.

\subsubsection{Specular Scattering}

Specular scattering occurs when the incident wavelength is many times smaller than the dimension of the scattering object, i.e. $k a \gg 1$. In this case the reflection of a wave can be approximated by ray theory i.e. the scattered wave front is a replica of the shape of the object as derived in [68]. The equation for the reflected intensity/incident intensity ratio for the wave scattered straight back at the source at an angle of $180^{\circ}$ relative to the incident wave (known as backscattering) is given by (2.31) [68].

$$
\frac{I_{r}}{I_{i}}=\frac{\pi b^{2}|R|^{2}}{4 \pi r^{2}}
$$

where $I_{\mathrm{r}}$ is the reflected intensity at a source distance $r$ from the scatterer, $I_{\mathrm{i}}$ is the incident intensity, $b$ is the amplitude of the incident wave, and $R$ is the pressure reflection coefficient given by (2.13) determined by the impedance mismatch between the propagating medium and the scattering sphere (the absolute value of $R$ is taken since only magnitude is required). The most important point to note from (2.31) is the ratio of the reflected intensity to the incident intensity is independent of the incident frequency.

\subsubsection{Diffusive Scattering}

At the other extreme diffusive scattering occurs when a scattering object is many times smaller than the insonifying wavelength i.e. $k a \ll<1$. Tissue is often modelled as consisting of small sub wavelength point scatterers. Examples in medical ultrasound are irresolvable blood cells or contrast agents consisting of tiny gas filled bubbles that are used to enhance the scattering from blood vessels for improved imaging [69]. Diffusive scattering from a point sphere is a classical problem that can be found in the literature as early as Lord Rayleigh [70], [71]. Using the result for a rigid sphere from [71], the backscatter intensity ratio is given by (2.32). 


$$
\frac{I_{r}}{I_{i}}=\frac{25 k^{4} a^{6}}{36 r^{2}}
$$

where $k$ is the wavenumber, $r$ is the distance from the scatterer, and $a$ is the radius of the scatterer. Comparing the result of (2.32) with that in (2.31) the significant difference is the scattered intensity here is proportional to the fourth power of frequency (via $\mathrm{k}^{4}$ ), and to the sixth power of the sphere radius.

\subsubsection{Diffractive Scattering}

Diffractive scattering corresponds to the range of $k a$ values that fall between the specular and diffusive scattering extremes, considered to be when the incident wavelength is comparable to the radius of the scattering sphere, i.e. $k a \cong 1$. Here the scattering profile from objects varies significantly with small changes in $k a$ as shown in the polar plots of Figure 2.18 (a)-(c).

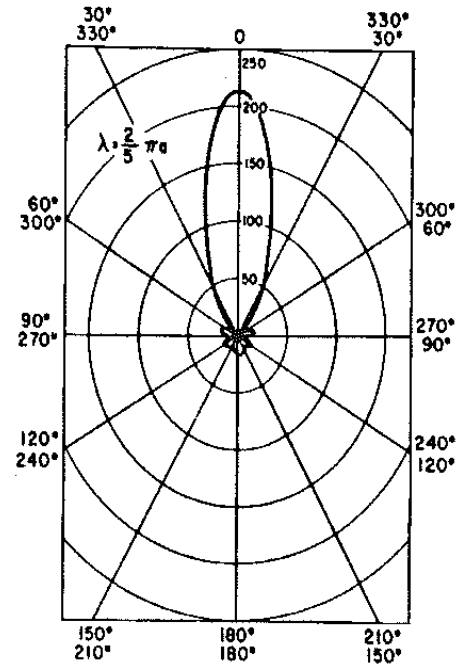

(a)

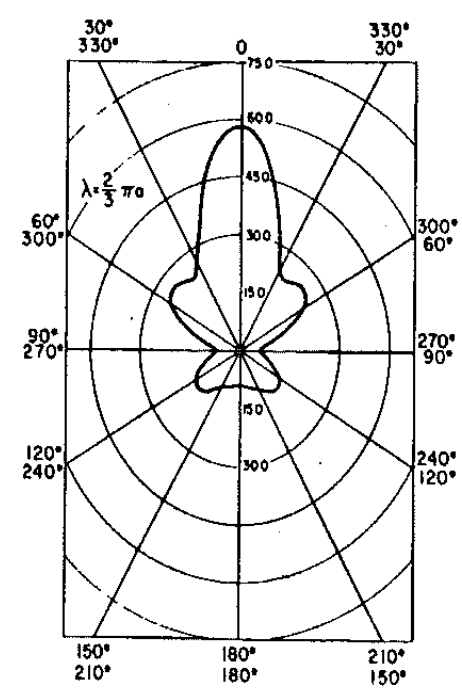

(b)

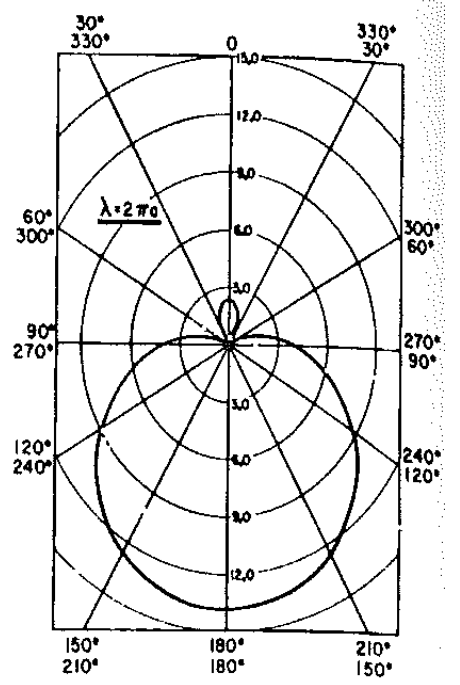

(c)

Figure 2.18 - Polar diagrams showing scattering from a rigid sphere for ka values of (a) 5, (b) 3 , and (c) $1.180^{\circ}$ corresponds to reflections direc ted back at the source, and $0^{\circ}$ corresponds to propagation directly away from the source [67]. 


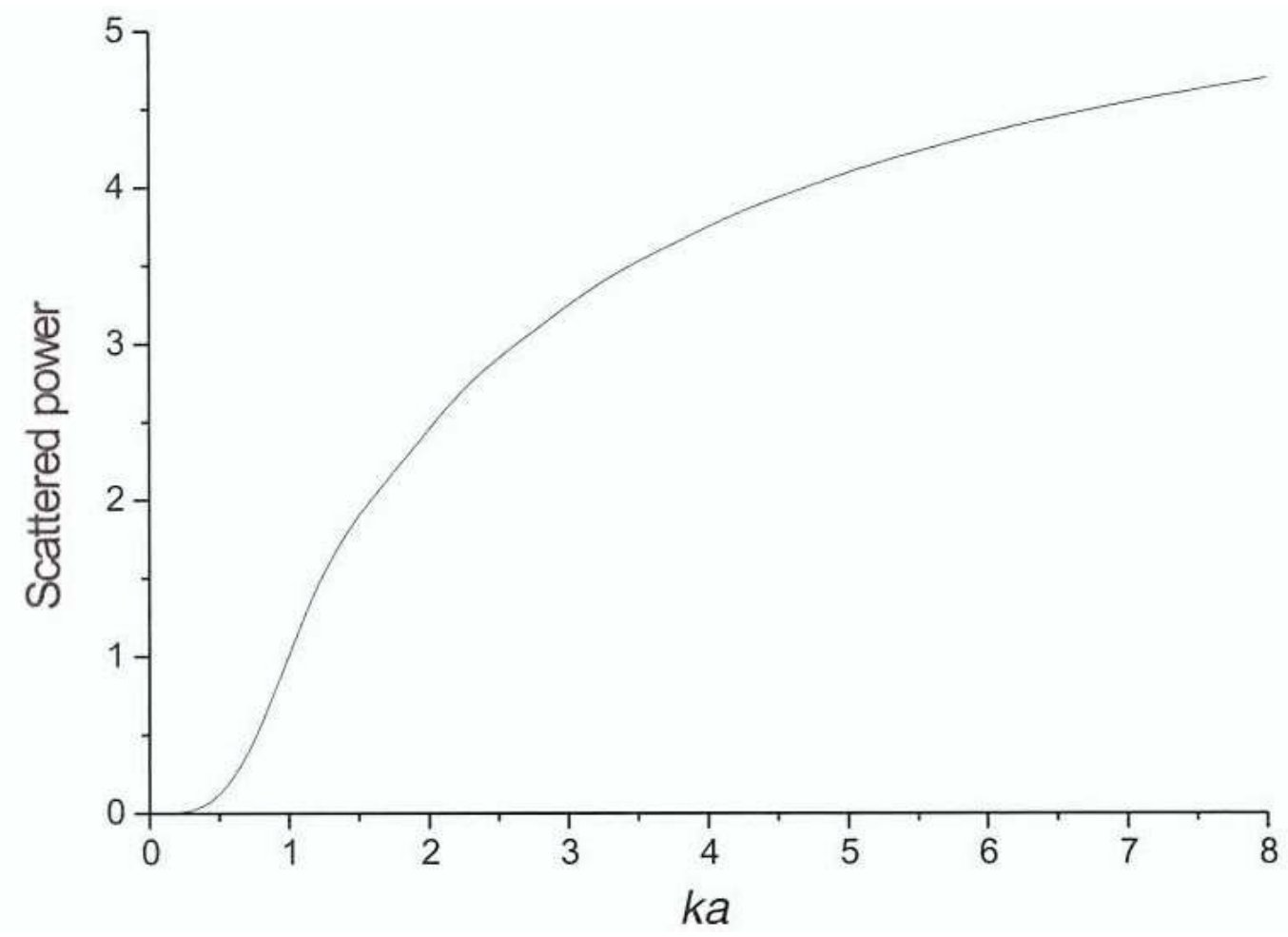

Figure 2.19 - Total scattered power from a sphere of radius a versus ka number.

In these diagrams $180^{\circ}$ corresponds to backscatter (straight back at the source), and $0^{\circ}$ corresponds to propagation along the direction of the incident wave. Figure 2.18 (a) is for $k a=$ 5 , (b) $k a=3$, and (c) $k a=1$. Since such a wide variety of scattering can exist in the diffractive scattering region due to the many different shapes and sizes of scatterers, it is difficult to formulate exact solutions for this range. An exact solution for elastic scattering from solid spheres and cylinders can be found in [72]-[74].

Figure 2.19 shows the total scattered power versus $k a$ number for a rigid sphere of radius $a$. For $k a<1$, the total scattered power rapidly increases with small increases in $k a$ consistent with the $f^{4}$ relationship associated with diffusive scattering. In the short wavelength case when $k a \gg 1$, the scattering is mainly specular and the scattered power saturates becoming frequency independent. The derivation of the expression for the scattered power can be found in [75]. 


\subsubsection{Absorption}

Absorption is the conversion of acoustic energy to heat due to viscosity in the medium. Classical absorption takes into account the transformation of acoustic energy into heat by material frictional forces and heat conduction. The transducers used in this work are high frequency (20 MHz and above) focussed ultrasonic immersion transducers. These are designed to operate immersed in water offset some distance from the target (see section 3.2.2). The waves generated travel 5-13 $\mathrm{mm}$ through water (depending on the device focal length), propagate through the target material, and then travel the same water distance again before being received. The absorption that occurs due to propagation in the water is significant and needs to be considered. The absorption of a material is characterised by its absorption coefficient $\alpha$, given by (2.33) [33].

$$
\alpha=\frac{\omega^{2}}{2 \rho v^{3}}\left(\frac{4}{3} \eta_{\text {shear }}+\eta_{\text {bulk }}+\frac{(\gamma-1) K}{c_{p}}\right)
$$

where $\eta_{\text {shear }}$ represents the shear viscosity of the absorber, $\eta_{b u l k}$ the bulk viscosity, $\gamma$ the specific heat ratio, $v$ the bulk longitudinal velocity, $c_{p}$ the specific heat, $\rho$ the density, $\omega$ the angular frequency, and $K$ is the thermal conductivity. For a liquid, the third term in the brackets is zero. Equation (2.33) shows the absorption coefficient is significantly dependent upon the frequency of the propagating wave. The amplitude of a wave $A$ in an attenuating material at a given depth $z$ is expressed as

$$
A=A_{0} \exp (-\alpha z)
$$

where $A_{0}$ is the initial amplitude of the wave before attenuation. Rearranging (2.34) in terms of $\alpha$ and taking the logarithm to express the result in $\mathrm{dB}$, Figure 2.20 plots the absorption in water in $\mathrm{dBmm}^{-1}$ as a function of frequency. This highlights the rapid increase in absorption with increasing frequency. At $50 \mathrm{MHz}$ absorption is $0.6 \mathrm{dBmm}^{-1}$, and at $100 \mathrm{MHz}$ this increases to $2 \mathrm{dBmm}^{-1}$. For the transducers with focal lengths of $13 \mathrm{~mm}$, at $100 \mathrm{MHz}$ the total attenuation is $52 \mathrm{~dB}\left(2 \mathrm{dBmm}^{-1} \times 13 \mathrm{~mm} \times 2\right)$, significantly attenuating the signal. 


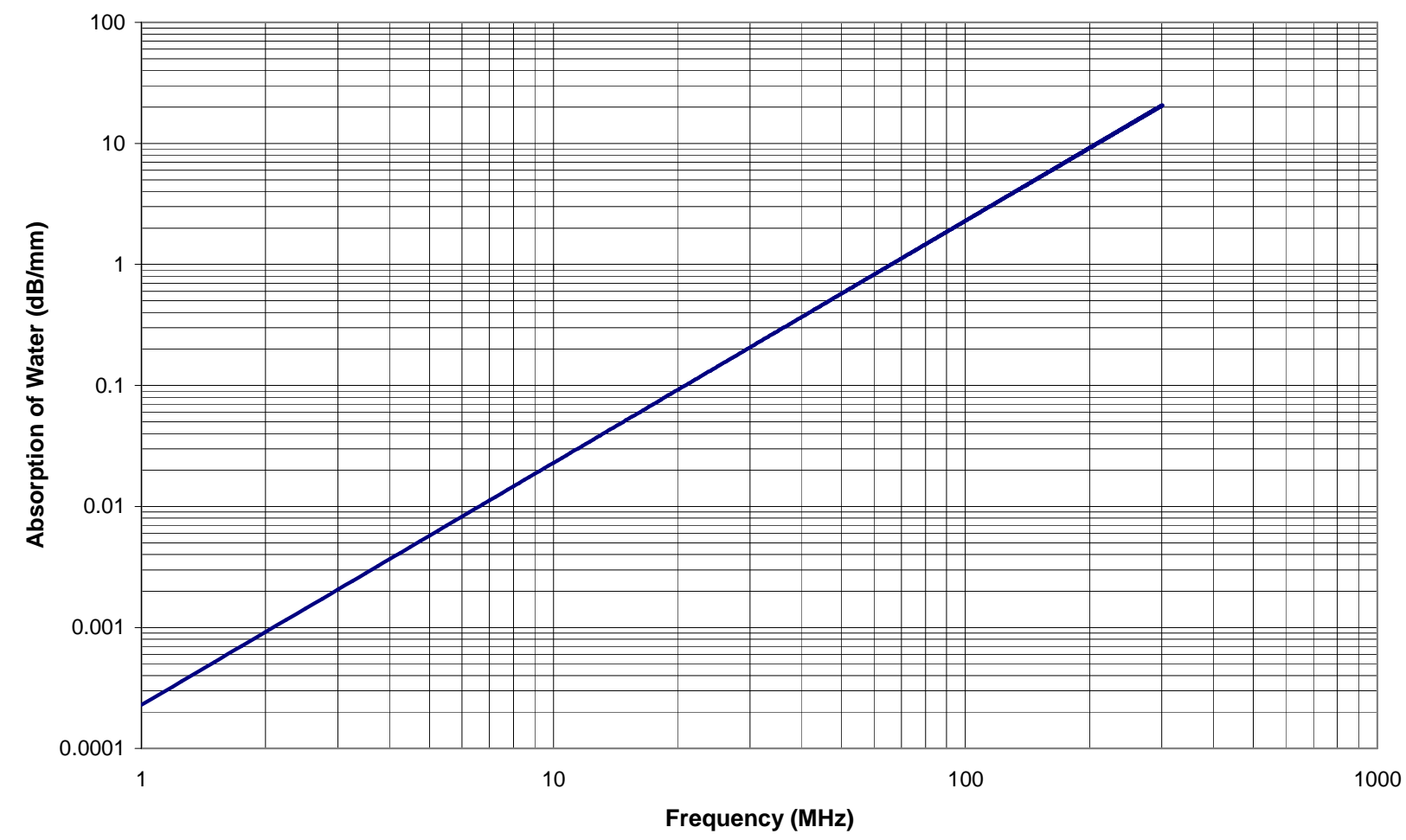

Figure 2.20 - Acoustic absorption in fresh water versus frequency. 


\section{Chapter 3}

\section{Design and Setup of Experimental Systems}

In this chapter the experimental methods/configurations used and the high frequency ultrasound system designed to perform the experimental measurements are described. Section 3.1 covers the transmission and pulse-echo measurement experimental configurations, with the high frequency transducers used in these configurations described in more detail in section 3.2. The custom designed high frequency electronics are described in section 3.3, followed by section 3.4 describing the custom system software. Finally the 3-axis positioning stage used for transducer alignment is briefly described in section 3.5.

\subsection{Experimental Configurations}

Two experimental configurations were used to characterize the sample materials in this work, a transmission measurement system and a pulse-echo measurement system. Sample materials included homogeneous solids, porous anodic alumina, and gelatinous homogeneous and anisotropic poly(vinyl alcohol) tissue mimicking phantoms.

\subsubsection{Transmission Measurement System}

A transmission measurement configuration was used to measure sample thickness, phase and group velocities, and attenuation. In this configuration separate transmitting and receiving transducers were used with the sample holder placed in between as shown in Figure 3.1. 
A burst waveform with user selectable parameters was generated on the system controlling PC using a custom graphical user interface (GUI) (section 3.4). This waveform was sent to an Agilent AFG3251 single channel arbitrary/function generator via an Ethernet network connection. The generated waveform was passed on to a transmitter that drove the transmitting transducer using either a custom made transmitter (section 3.3.2) or a commercial power amplifier (section 3.3.4). The transmitting and receiving transducers were $50 \mathrm{MHz}, 110 \mathrm{MHz}$, or $175 \mathrm{MHz}$ pairs (section 3.2). The SYNC signal from the function generator synchronised the oscilloscope capture for triggering. The transmitting transducer was positioned using a precision 3-axis linear actuator system (section 3.5). This positioning system also contained a rotational stage to incline the transmitting transducer at various angles. A custom-made low noise receiver circuit (section 3.3.3) amplified the signals received from the receiving transducer. These were captured by a Tektronix TDS3034 8-bit digital phosphor oscilloscope and were downloaded to the PC for signal processing.

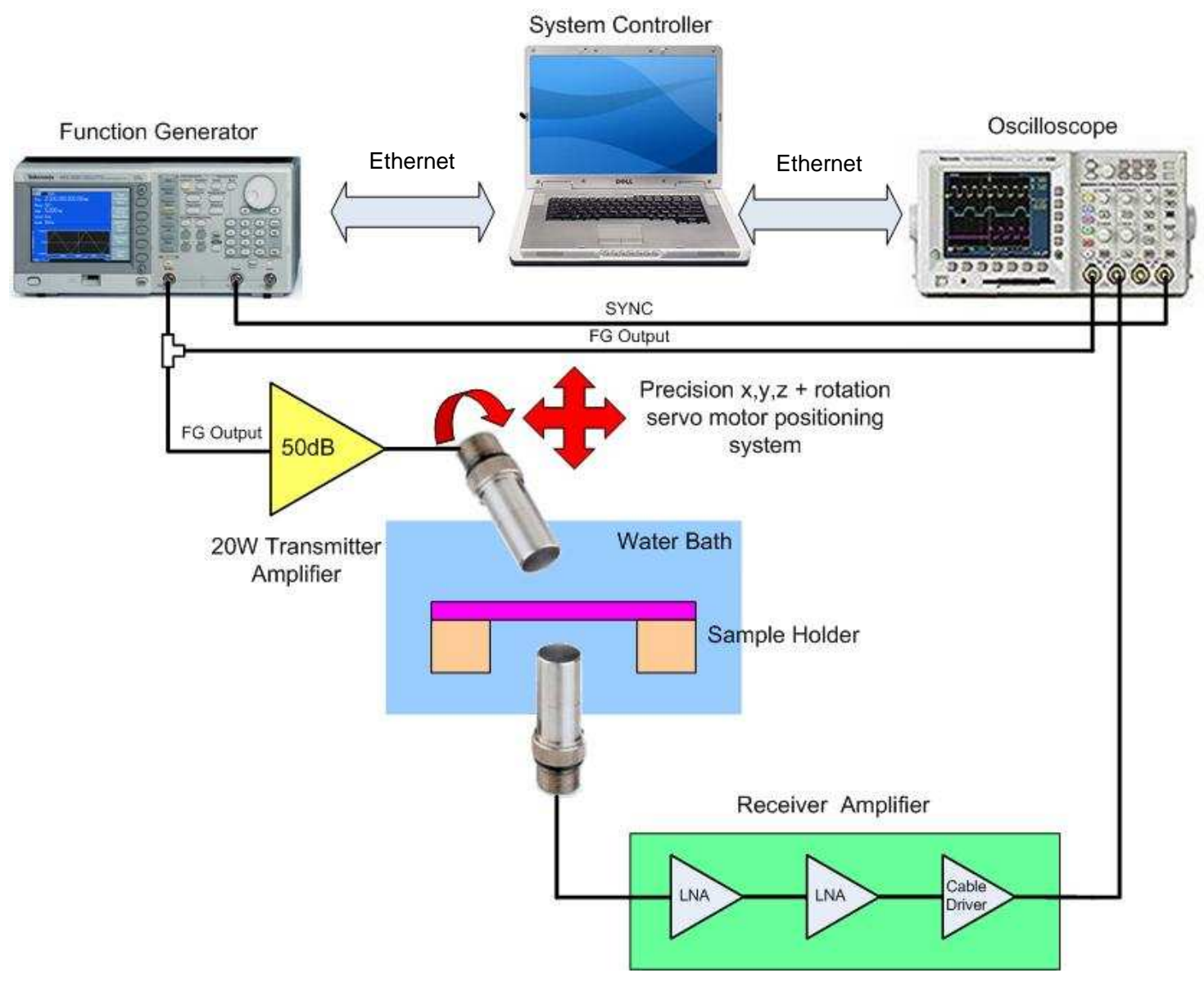

Figure 3.1 - Transmission measurement experimental configuration. 
The transducers and sample were immersed in a water bath during measurement. Figure 3.2 shows the water bath setup for a transmission measurement at normal incidence. The distances of the transducers from the sample were adjusted until the sample was inside the focal zones. The top transducer position was then adjusted in the horizontal axes until a maximum signal was received, corresponding to ideal alignment. Sample thickness, velocity, and attenuation were measured using ultrasound spectroscopy methods as detailed in the literature [78]-[81].

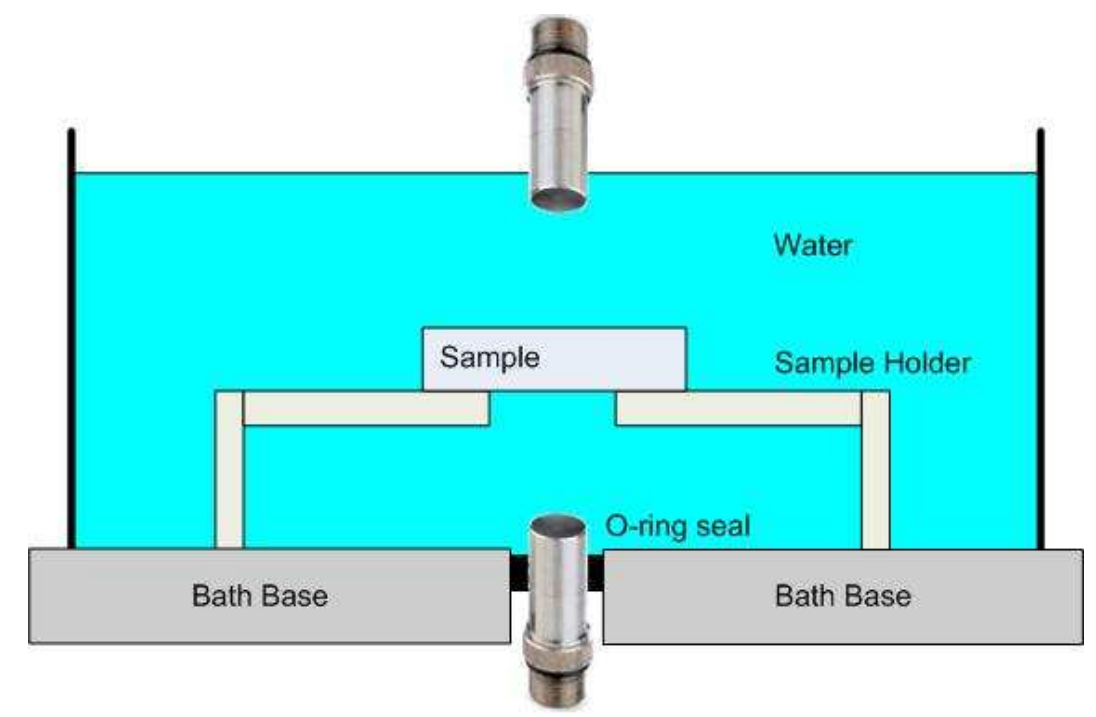

Figure 3.2 - Transmission measurement water bath setup.

\subsubsection{Sample Thickness}

For rigid samples a micrometer could be used to measure sample thickness. For soft samples the thickness was calculated by performing three time-of-flight ultrasonic measurements as shown in Figure 3.3.

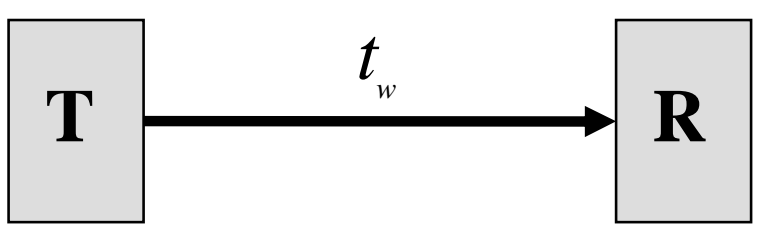

(a)

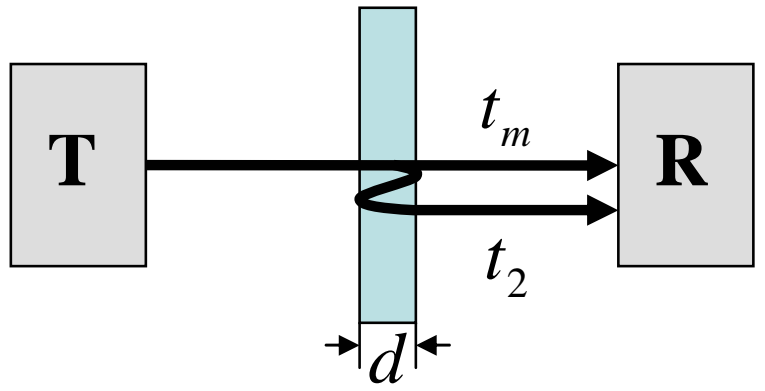

(b)

Figure 3.3 - Time of flight measurements (a) without sample, and (b) with sample in place. 
Transmission times $t_{\mathrm{m}}$ and $t_{\mathrm{w}}$ were the measured pulse transmission times from transmitter to receiver with and without the sample present respectively, and time $t_{2}$ was the measured transmission time with the sample in place after a single reverberation within the sample. These measurements were made by performing cross correlation using the known transmitted waveform and received signals to determine these times with precision (see section 3.4). The sample thickness $d$ was found from

$$
d=\left(\frac{v_{w}}{2}\right)\left[2\left(t_{w}-t_{m}\right)+\left(t_{2}-t_{m}\right)\right]
$$

where $v_{\mathrm{w}}$ is the speed of sound in water. The theoretical speed of sound in pure water at a given temperature $T$ was found using a fifth order polynomial [82], [83]. The temperature of the water bath was monitored constantly, and no velocity measurement was performed until the water temperature had settled to room temperature.

\subsubsection{Sample Velocity}

If the sample thickness $d$ is known, the phase velocity $v_{\mathrm{p}}(f)$ in a sample can be calculated using the phase spectrums captured with the sample in place $\phi_{\mathrm{m}}(f)$ and without the sample in place $\phi_{\mathrm{w}}(f)$ as shown in Figure 3.4.
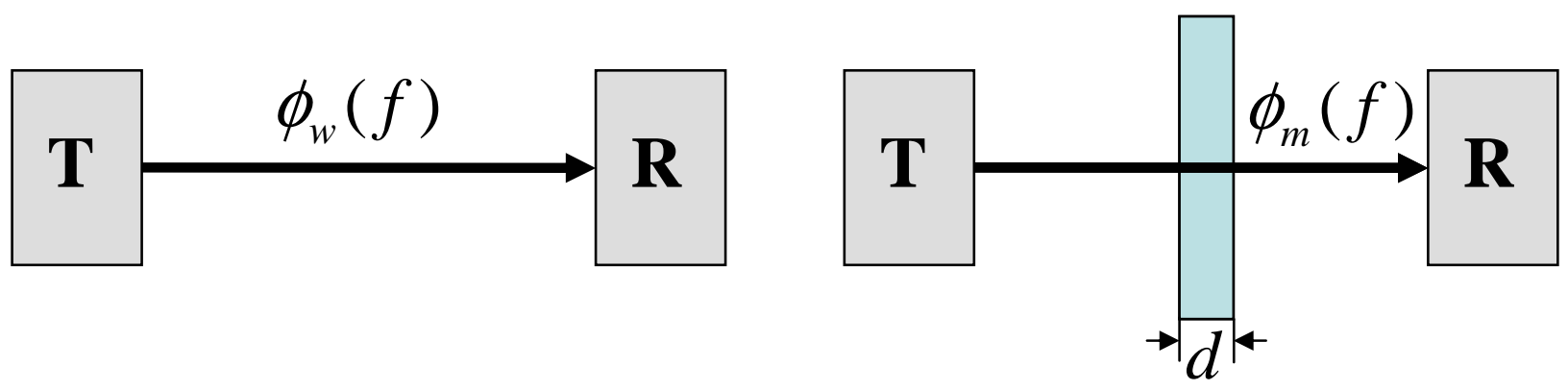

Figure 3.4 - Phase measurements to calculate sample velocity for known sample thickness $d$.

The phase spectrums were found by performing FFTs on the captured waveforms then finding the arguments of these since variations in phase in the frequency domain are equivalent to time shifts in the time domain. The phase velocity was found for all frequencies using equation (3.2). The unwrapped phase was used for the calculation which was implemented in software. 
The sample had to be sufficiently thick so that $\phi_{\mathrm{m}}$ could be separated/time gated from reverberant waves that occur in the sample.

$$
v_{p}(f)=\frac{v_{w}}{1+\frac{\left[\phi_{w}(f)-\phi_{m}(f)\right] v_{w}}{2 \pi f d}}
$$

The group velocity (wave envelope velocity) of a wave $v_{\mathrm{g}}$ was found using $t_{\mathrm{w}}$ and $t_{\mathrm{m}}$ in equation (3.3). This equation however is only applicable for non-dispersive media.

$$
v_{g}=\frac{v_{w}}{1+\frac{\left(t_{w}-t_{m}\right) v_{w}}{d}}
$$

\subsubsection{Sample Attenuation Coefficient}

The attenuation coefficient $\alpha(f)$ of a sample was found using the FFT magnitudes of two waveforms having passed through samples of identical composition but with differing thickness. This is shown in Figure 3.5.
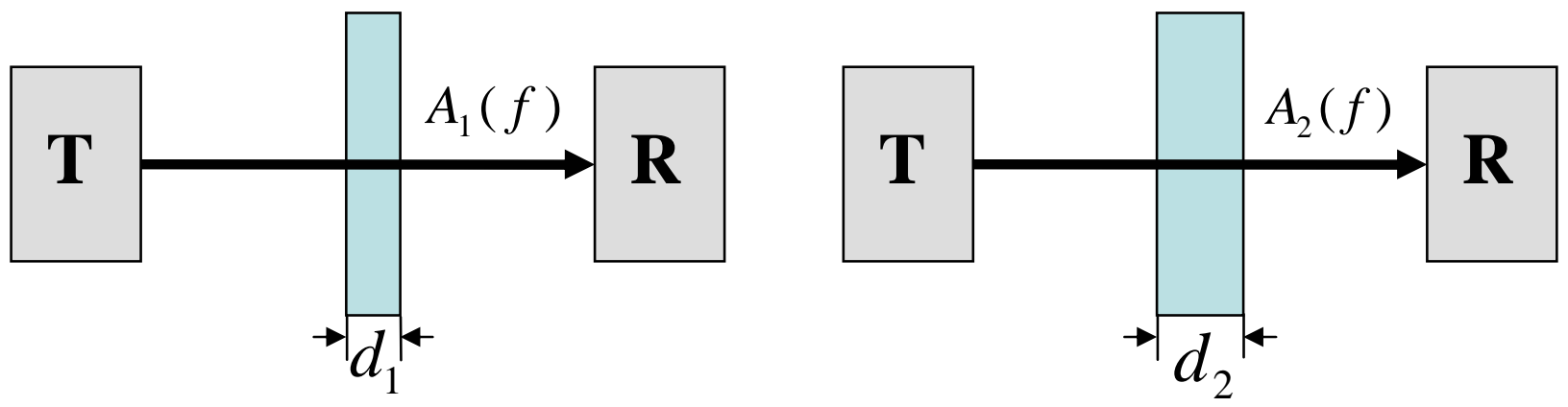

Figure 3.5 - Amplitude measurements to calculate sample attenuation coefficient.

The first measurement was made with a sample of thickness $d_{1}$ in place, the second with sample thickness $d_{2}>d_{1}$. The long focal lengths of the transducers relative to the sample thickness and the comparatively small attenuation in the water meant the difference in water path lengths was negligible. This measurement assumes identical sample structure so samples had to have been 
prepared using the same process. Using the measured FFT magnitude spectra $A_{1}(f)$ and $A_{2}(f)$ from Figure 3.5, the attenuation coefficient is then given by

$$
\alpha(f)=\frac{-20 \log \left(\frac{A_{2}(f)}{A_{1}(f)}\right)}{\left(d_{2}-d_{1}\right)}
$$

\subsubsection{Pulse Echo Measurement System}

The pulse echo configuration was used to measure the acoustic impedance of a sample. In this configuration, shown in Figure 3.6, a single transducer was used for both transmit and receive. The sample was placed on an aluminium base to provide a strong acoustic reflection from the bottom surface. The incident wave was generated using the same method as was described for the transmission measurement (section 3.1.1).

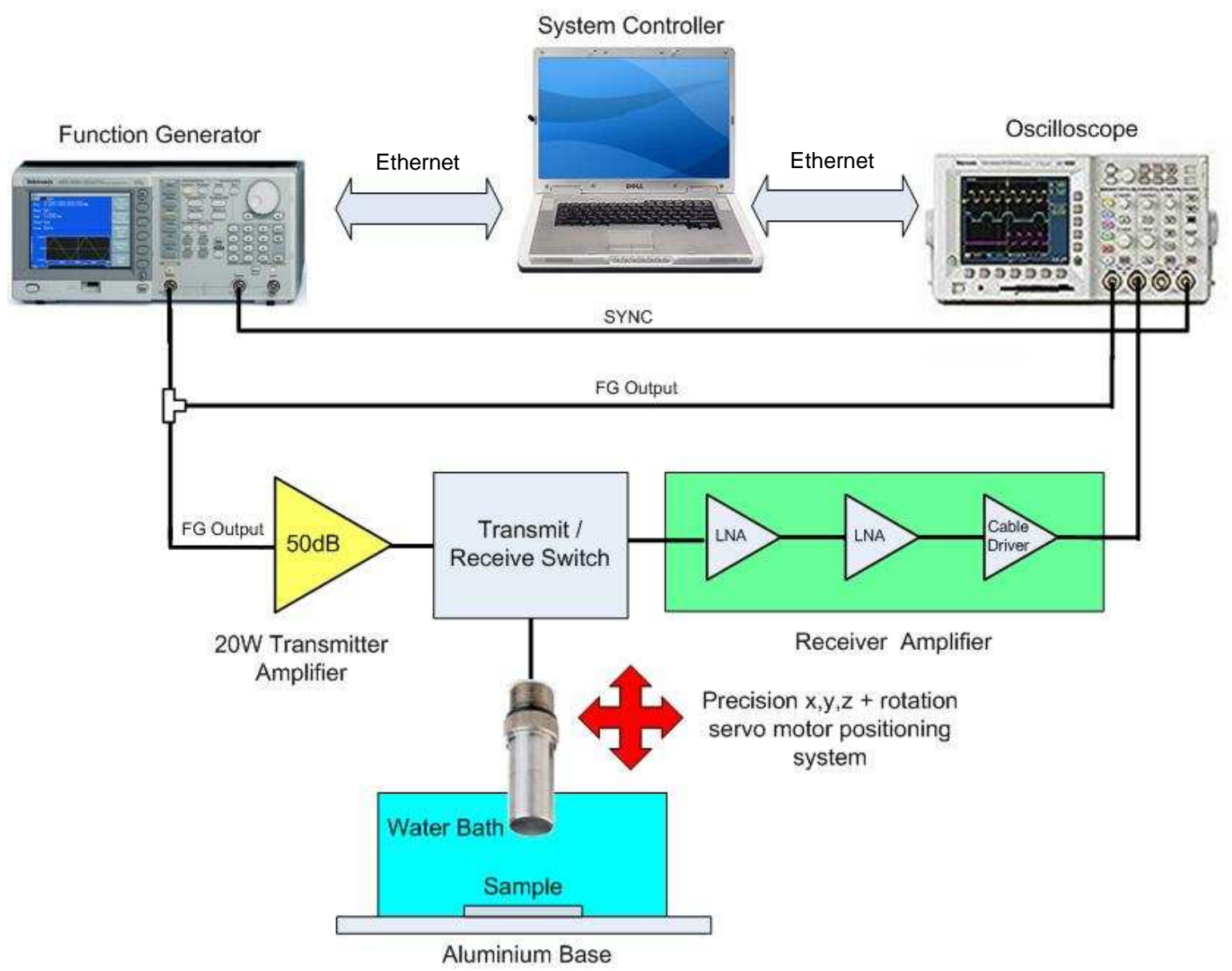

Figure 3.6 - Pulse echo measurement experimental configuration. 
The SYNC signal as in the transmission measurement setup synchronised the oscilloscope capture and in addition gated the transmit/receive switch (section 3.3.6). This switch isolated the transmitter or receiver circuits from the transducer depending on whether the system was transmitting or receiving. As with the transmission setup, a low noise receiver circuit amplified the signals received from the transducer before capture by the oscilloscope.

\subsubsection{Sample Acoustic Impedance}

The acoustic impedance of a sample was found using a similar method to that used in [84]. This method measures the amplitude reflected from an unknown sample and compares it to the amplitude reflected from a material of known acoustic impedance e.g. steel or aluminium. These measurements are shown in Figure 3.7.
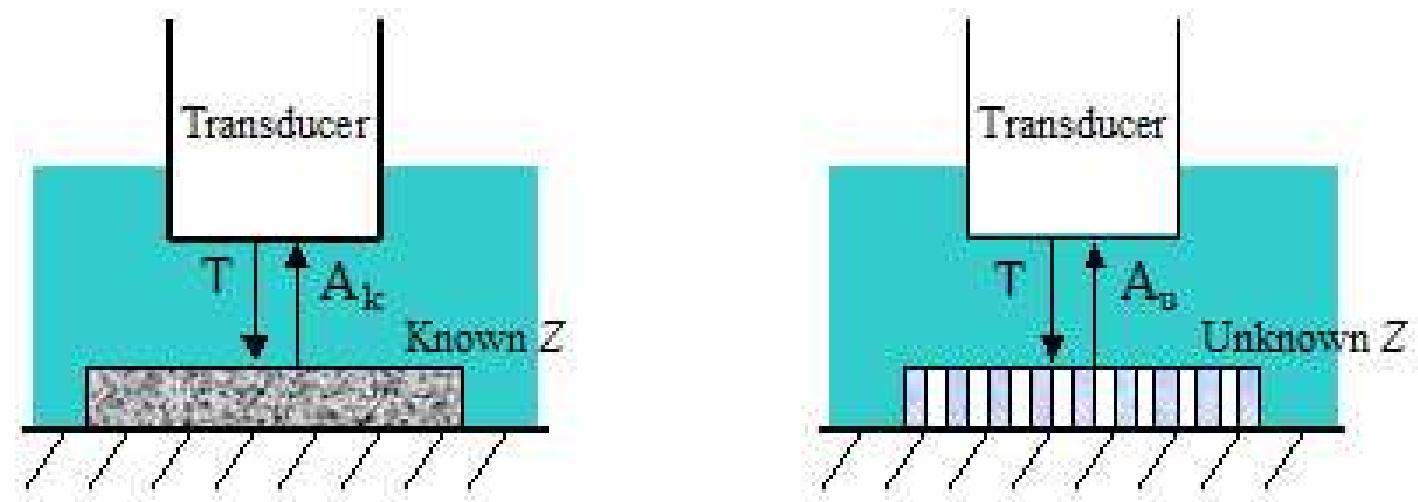

Figure 3.7 - Pulse echo measurements for unknown sample acoustic impedance.

For both measurements the same transducer was used, so the transmitted signal $T$ was the same for both measurements, incurring the same transducer transfer function on transmit and receive. The distance from transducer to sample was equal to the focal length of the transducer. This was adjusted until a maximum reflected signal was received confirming when the sample was in the transducer focal zone. The measured amplitudes as reflected from the known and unknown samples were given by

$$
\begin{aligned}
& A_{k}=T \alpha_{w}^{2} R_{k} \\
& A_{u}=T \alpha_{w}^{2} R_{u}
\end{aligned}
$$


where $\alpha_{\mathrm{w}}$ was the absolute attenuation coefficient of water, and $R_{\mathrm{k}}$ and $R_{\mathrm{u}}$ the pressure reflection coefficients between the water/known and water/unknown sample interfaces respectively given by equation (2.13). The water attenuation coefficient was squared as attenuation occurs in both directions. Taking the ratio of the measured amplitudes $A_{\mathrm{k}}$ and $A_{\mathrm{u}}$, this is simply equal to the ratio of the reflection coefficients $R_{\mathrm{k}}$ and $R_{\mathrm{u}}$.

$$
\frac{A_{k}}{A_{u}}=\frac{R_{k}}{R_{u}}
$$

Rewriting $R_{\mathrm{u}}$ in terms of the unknown acoustic impedance $Z_{\mathrm{u}}$ and the acoustic impedance of water $Z_{\mathrm{w}}$, the impedance $Z_{\mathrm{u}}$ is given by

$$
Z_{u}=Z_{w} \frac{A_{k}+A_{u} R_{k}}{A_{k}-A_{u} R_{k}}
$$

The water temperature was again constantly monitored providing accurate values for the acoustic impedance of the water and to provide an accurate result for the unknown sample impedance.

\subsection{High Frequency Ultrasonic Transducer Characteristics}

\subsubsection{Piezoelectric Transducer Construction}

An ultrasonic piezoelectric transducer uses the piezoelectric effect to convert an alternating potential difference applied across the crystal into a mechanical wave due to the repeated deformation of the structure. The same applies in reverse where an applied mechanical stress to the crystal causes the generation of a potential difference across the crystal. This will occur for a certain range of frequencies over which the transducer is designed to operate. A cross section of a typical contact piezoelectric ultrasonic transducer is shown in Figure 3.8. 


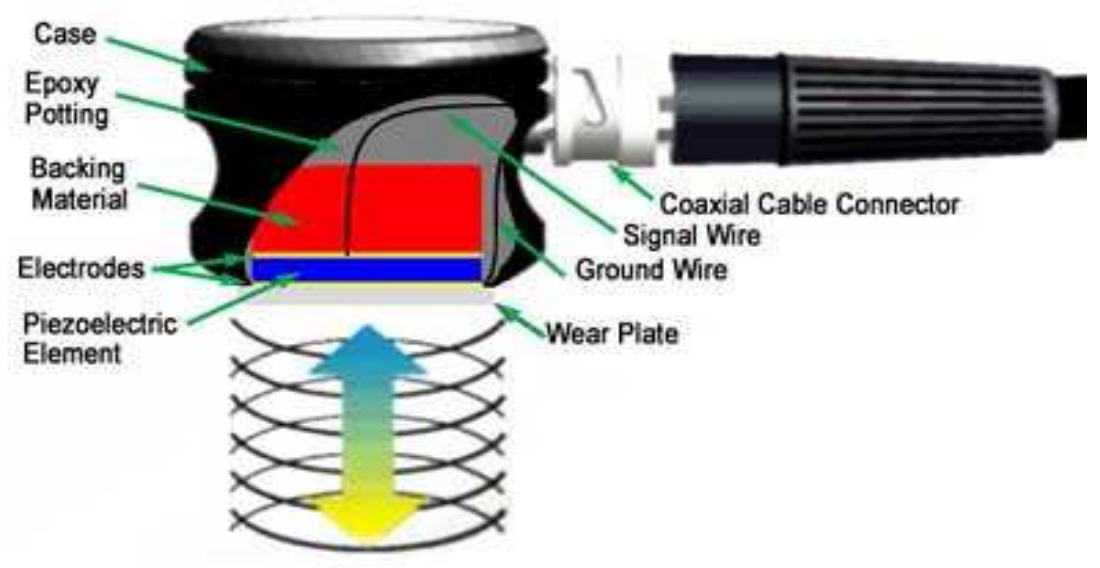

Figure 3.8 - Cross section of a contact piezoelectric transducer [76].

The fundamental components of a typical piezoelectric transducer are the following:

1) The backing material provides damping for the transducer. A highly damped transducer reduces ringing time thus producing sharp pulses, resulting in broad bandwidth signals, but with low energy. Broad bandwidth implies improved axial resolution - the ability to resolve targets vertically separated along the vertical or depth axis. For a lightly damped transducer the reverse applies (low bandwidth, higher energy pulses).

2) The piezoelectric element is the active layer that responds to the applied potential difference by vibrating, generating a pressure wave. This element is connected to the transducer signal wires via electrodes that connect across the element as shown. This construction is similar to a capacitor, giving rise to the typical $1 / f$ characteristic associated with piezoelectric devices.

3) The matching layer (not shown here) is used to acoustically match the target material to the transducer active element. This is inserted between the active element and the wear plate. This improves energy transmission as was discussed in section 2.3.3.

\subsubsection{Immersion Transducers}

Ultrasonic immersion transducers were used in this work, which are focussed, high frequency, wide bandwidth (broadband), piezoelectric devices. Immersion ensures uniform coupling of energy from the transducer into the sample of interest. These transducers contain an optically ground spherical silica glass lens that: 
- Acts as an acoustic delay line. This ensures the device stops transmitting before an echo is received back for a pulse echo measurement.

- Focuses the transducer beam, concentrating the ultrasound beam to a spot improving both lateral resolution and sensitivity to targets. Maximising transducer sensitivity was important since high frequency corresponds to high acoustic absorption (see section 2.5.2).

- Acoustically matched the transducer active element to water in order to ensure maximum transfer of energy from the transducer into the sample (see section 2.3.3).

\subsubsection{Transducer Frequencies}

Pairs of immersion transducers with centre frequencies of $50 \mathrm{MHz}, 110 \mathrm{MHz}$, and $175 \mathrm{MHz}$ were used in this work; model numbers V390, V3802, and V3965 from Olympus Panametrics. These were broad bandwidth devices that allowed pulse compression to be used for improved axial resolution. Figure 3.9, Figure 3.10, and Figure 3.11 show the typical frequency responses provided from Olympus for the three different types of transducers. Responses vary slightly between devices with same part numbers. The broadband nature of the devices can be observed where the $50 \mathrm{MHz}$ transducer has a $-6 \mathrm{~dB}$ bandwidth of $35 \mathrm{MHz}$, the $110 \mathrm{MHz}$ transducer a -6 $\mathrm{dB}$ bandwidth of $96 \mathrm{MHz}$, and the $175 \mathrm{MHz}$ transducer a $-6 \mathrm{~dB}$ bandwidth of $142 \mathrm{MHz}$.

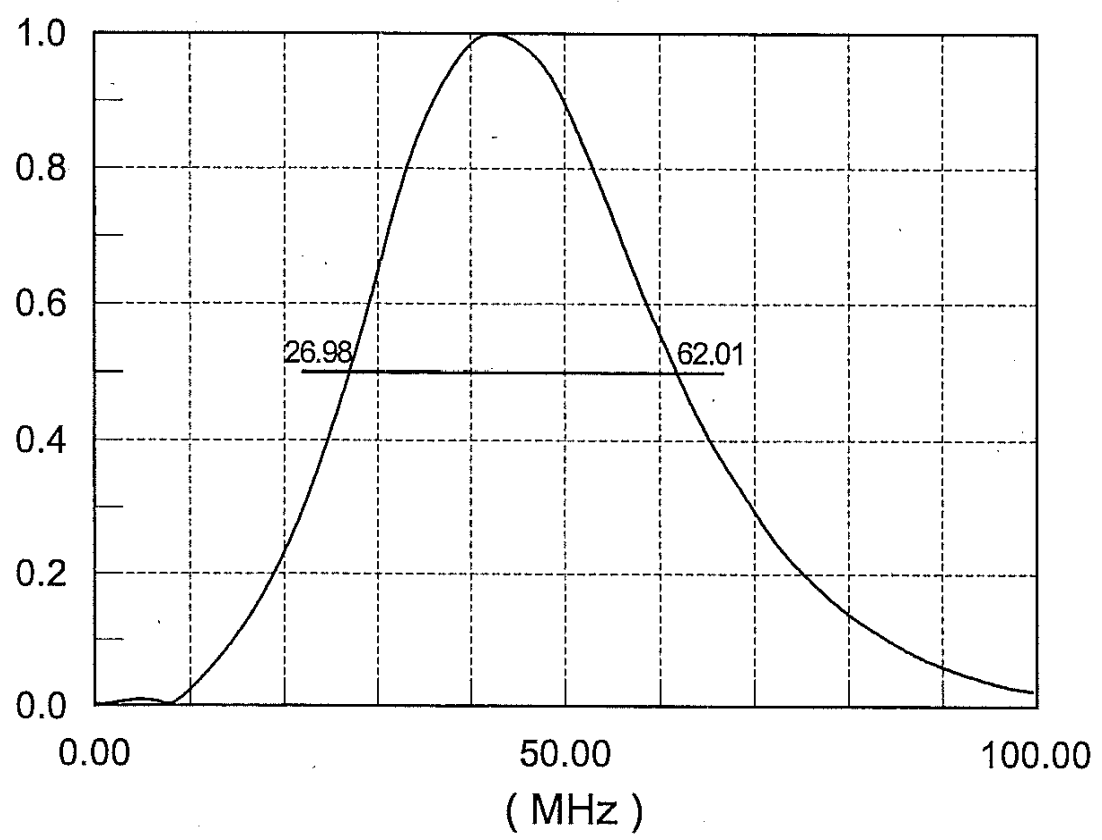

Figure 3.9 - Typical frequency response for the $\mathrm{V} 39050 \mathrm{MHz}$ transducers [85]. 


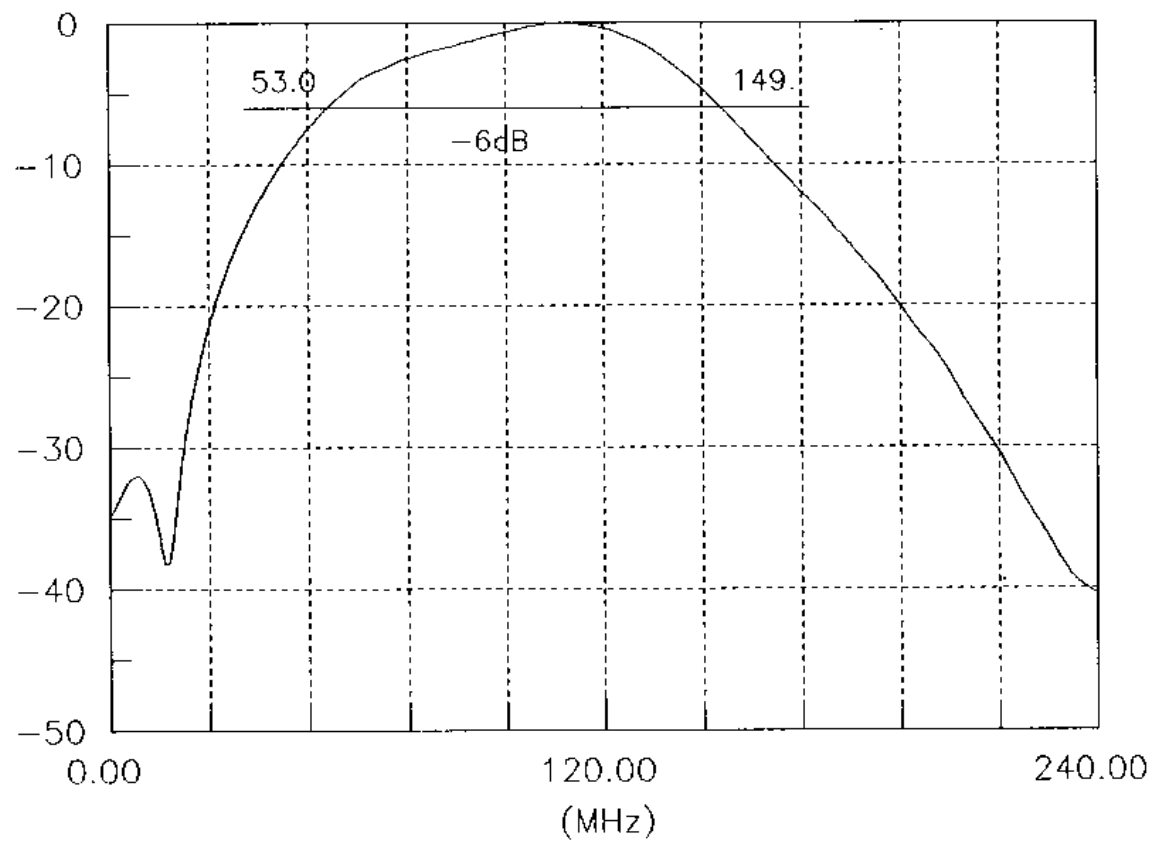

Figure 3.10 - Typical frequency response for the $\mathrm{V} 3802110 \mathrm{MHz}$ transducers [85].

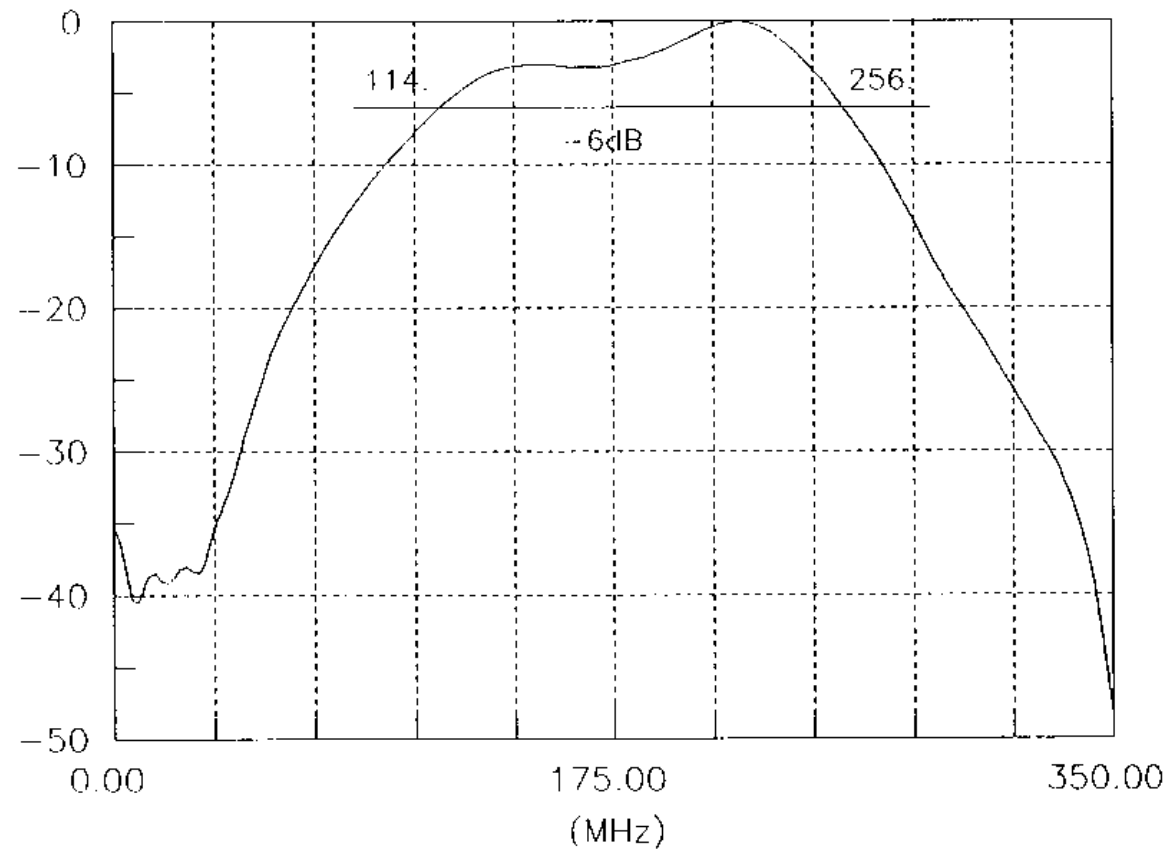

Figure 3.11 - Typical frequency response for the V3965 $175 \mathrm{MHz}$ transducers [85]. 


\subsubsection{Transducer Impedance}

The impedances of the transducers were measured using an Agilent 4291B $1 \mathrm{MHz}-1.8 \mathrm{GHz}$ impedance analyser. The impedance analyser was calibrated using the full procedure as described in [86] for accurate measurements. By measuring the transducer impedance characteristics, the transmitter output stage was designed to be electronically matched to the transducer impedance for improved power transfer.

\subsubsection{50 MHz Transducers}

The magnitude and phase characteristics of the two V390 $50 \mathrm{MHz}$ devices are shown in Figure 3.12 from 1-100 MHz. The characteristics of the two devices were very similar, however the measured characteristics strayed from those expected for a piezoelectric transducer. The physical construction of a typical piezoelectric transducer is similar to a capacitor (see section 3.2.1), displaying a $1 / f$ characteristic with small resistive deviations around the active region. Instead the measured plots revealed highly resistive devices measuring $50 \mathrm{ohm}$ at DC, indicating a resistor was placed across each input, which was not stated in the manufacturers datasheet.

The datasheet measurements were performed without the resistor present showing a highly capacitive transducer. The addition of the resistor caused significant changes to the transducer performance, improving matching of the transducers to the $50 \mathrm{ohm}$ cable and resulting in a more resistive device, making it easier to drive (a capacitive load means most of the driving energy is stored in an electric field). The measured magnitudes at $50 \mathrm{MHz}$ were approximately 33 ohms for both devices. Between 25 and $75 \mathrm{MHz}$ bumps in the plots can be observed corresponding to reverberations in the transducer delay lines, identifying the active frequencies and broadband characteristics.

\subsubsection{110 MHz Transducers}

The magnitudes and phases for the two V3208 $110 \mathrm{MHz}$ transducers are shown in Figure 3.13 from 1-250 MHz. Figure 3.13 confirms both of these transducers also possessed resistors across their inputs with magnitudes of $53 \mathrm{ohms}$ at DC. At $110 \mathrm{MHz}$, the magnitude of both transducers was $45 \mathrm{ohms}$, very well matched to the $50 \mathrm{ohm}$ electronics of the system. 


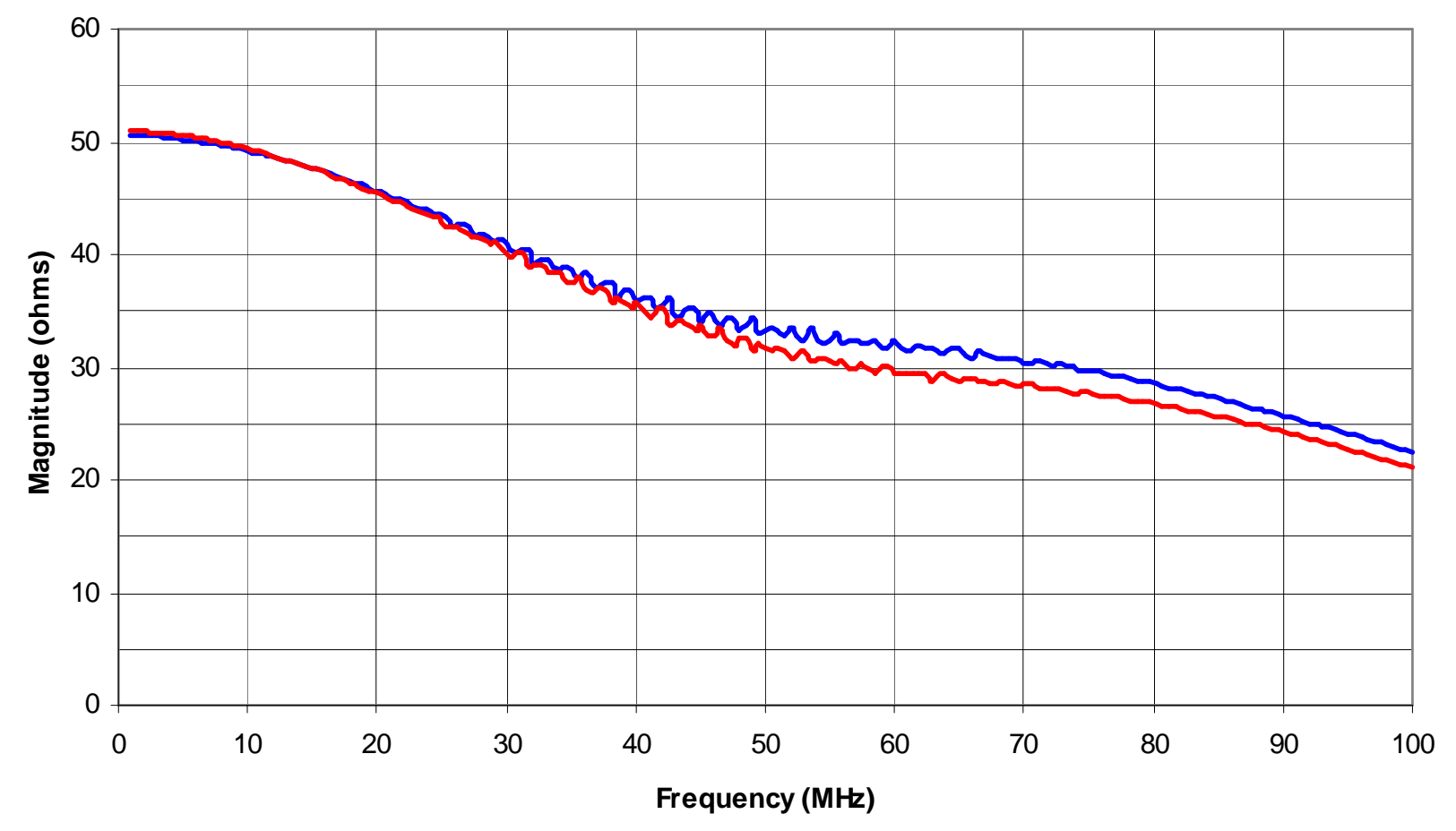

(a)

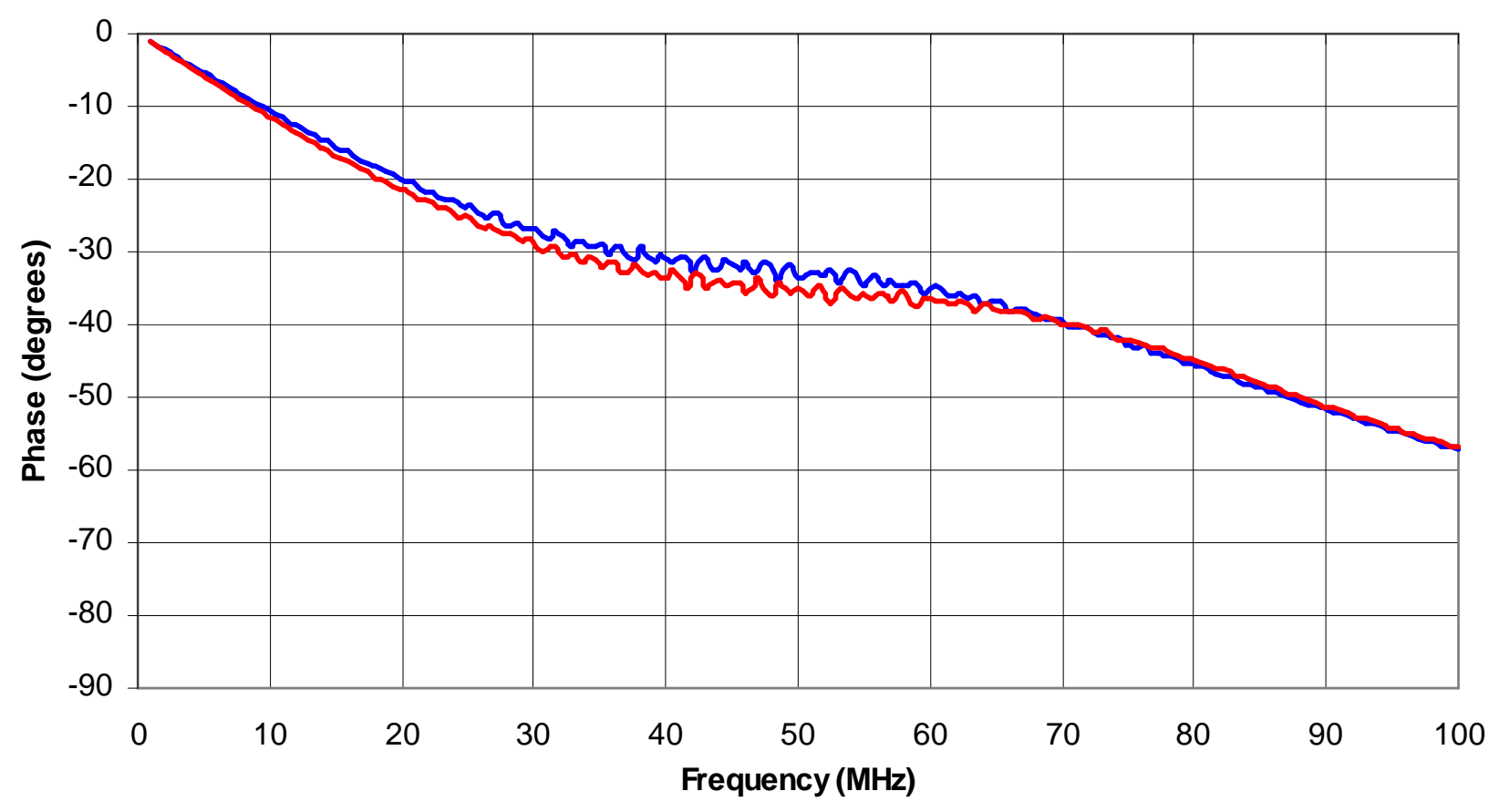

(b)

Figure 3.12 - Magnitude (a) and phase (b) characteristics of the two $50 \mathrm{MHz}$ transducers. 


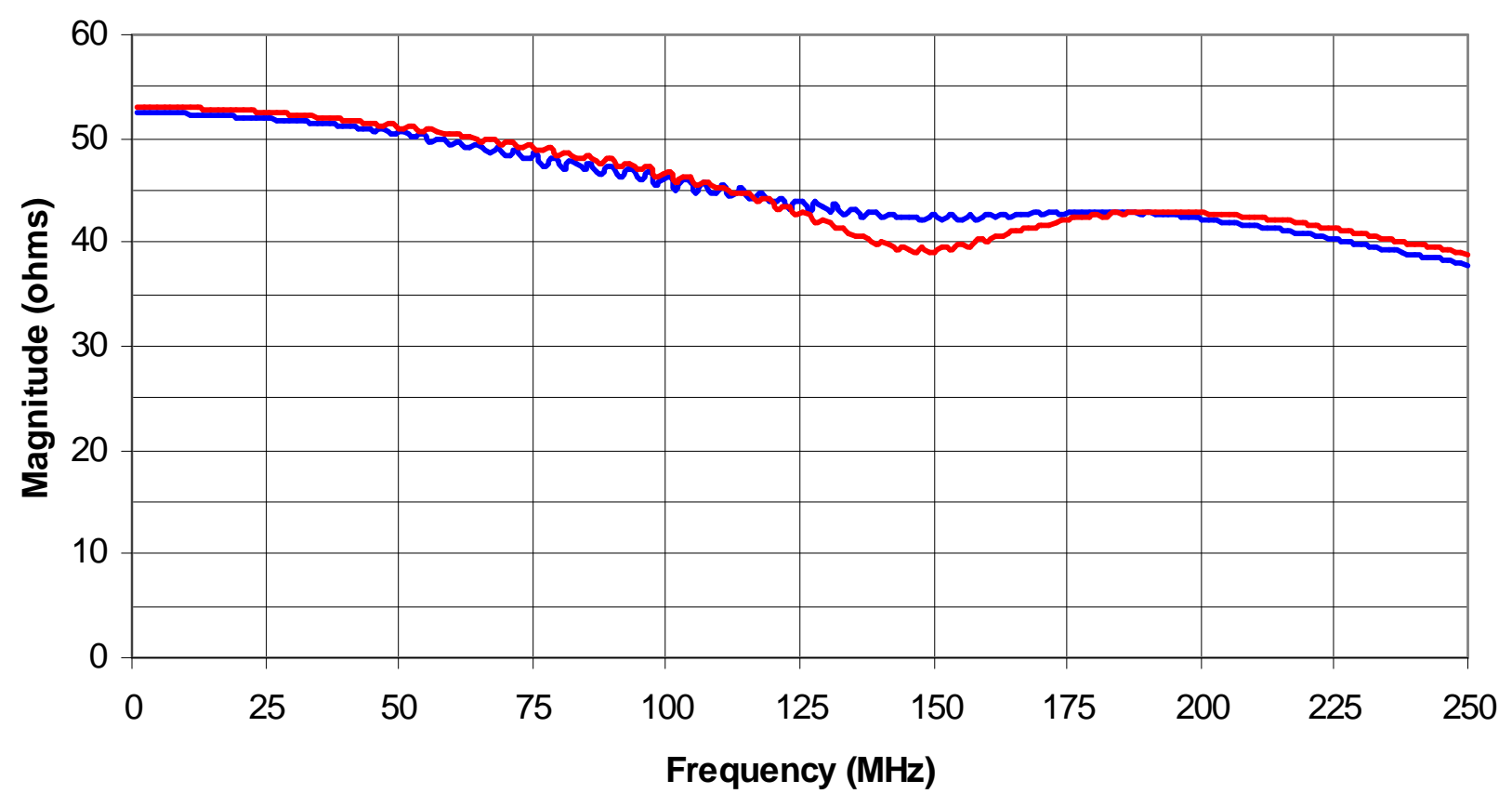

(a)

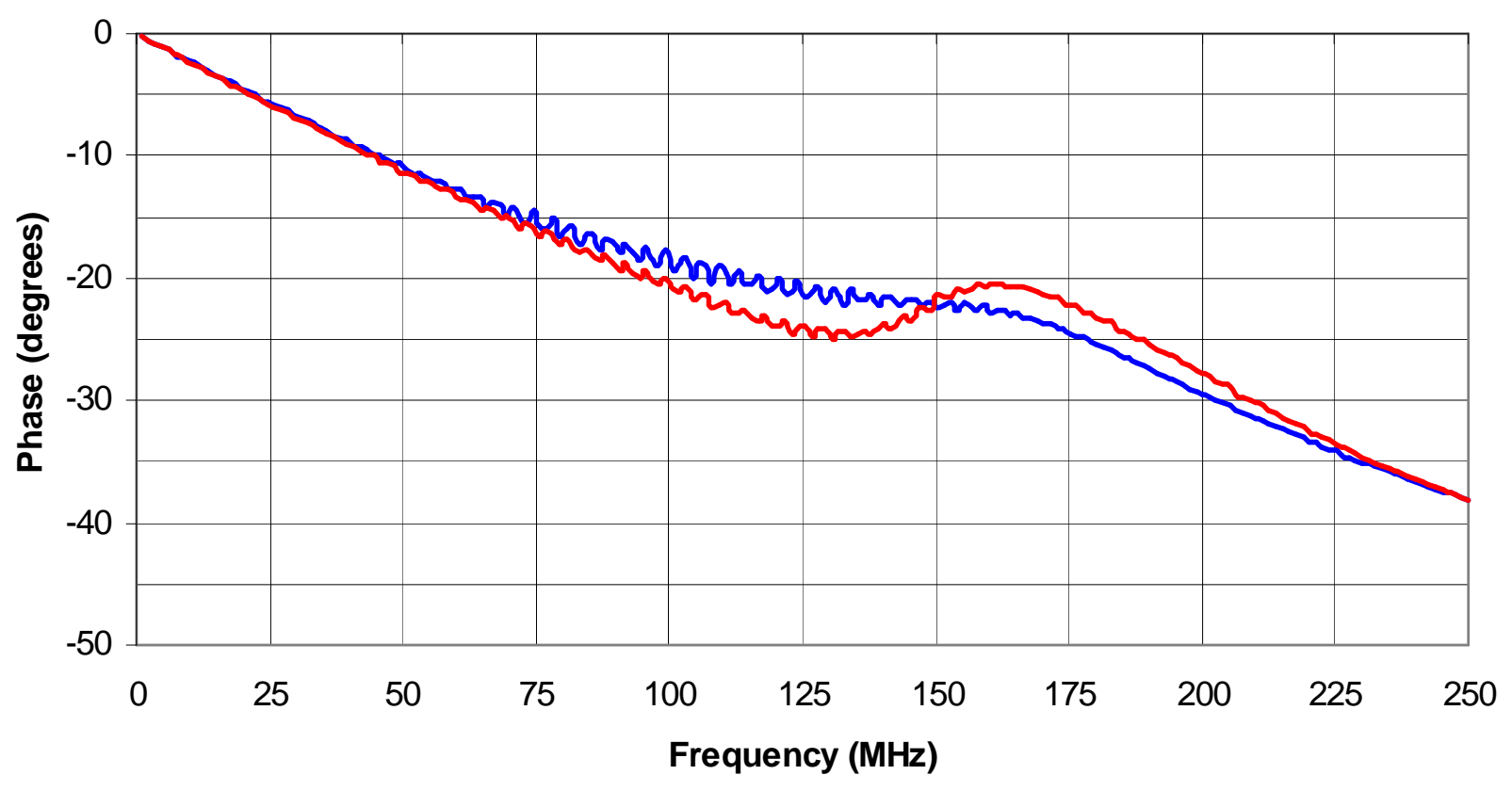

(b)

Figure 3.13 - Magnitude (a) and phase (b) characteristics of the two $110 \mathrm{MHz}$ transducers. 


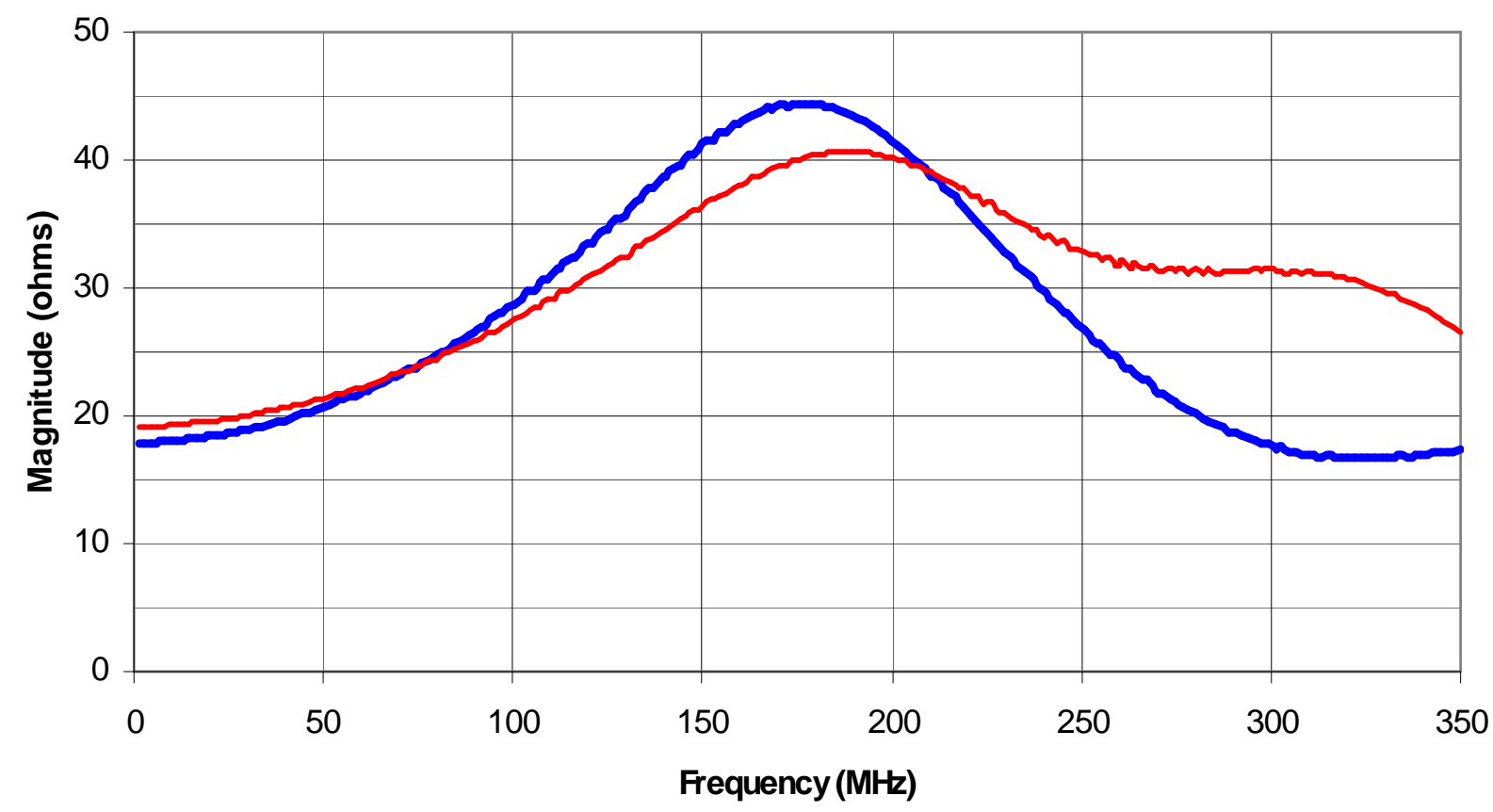

(a)

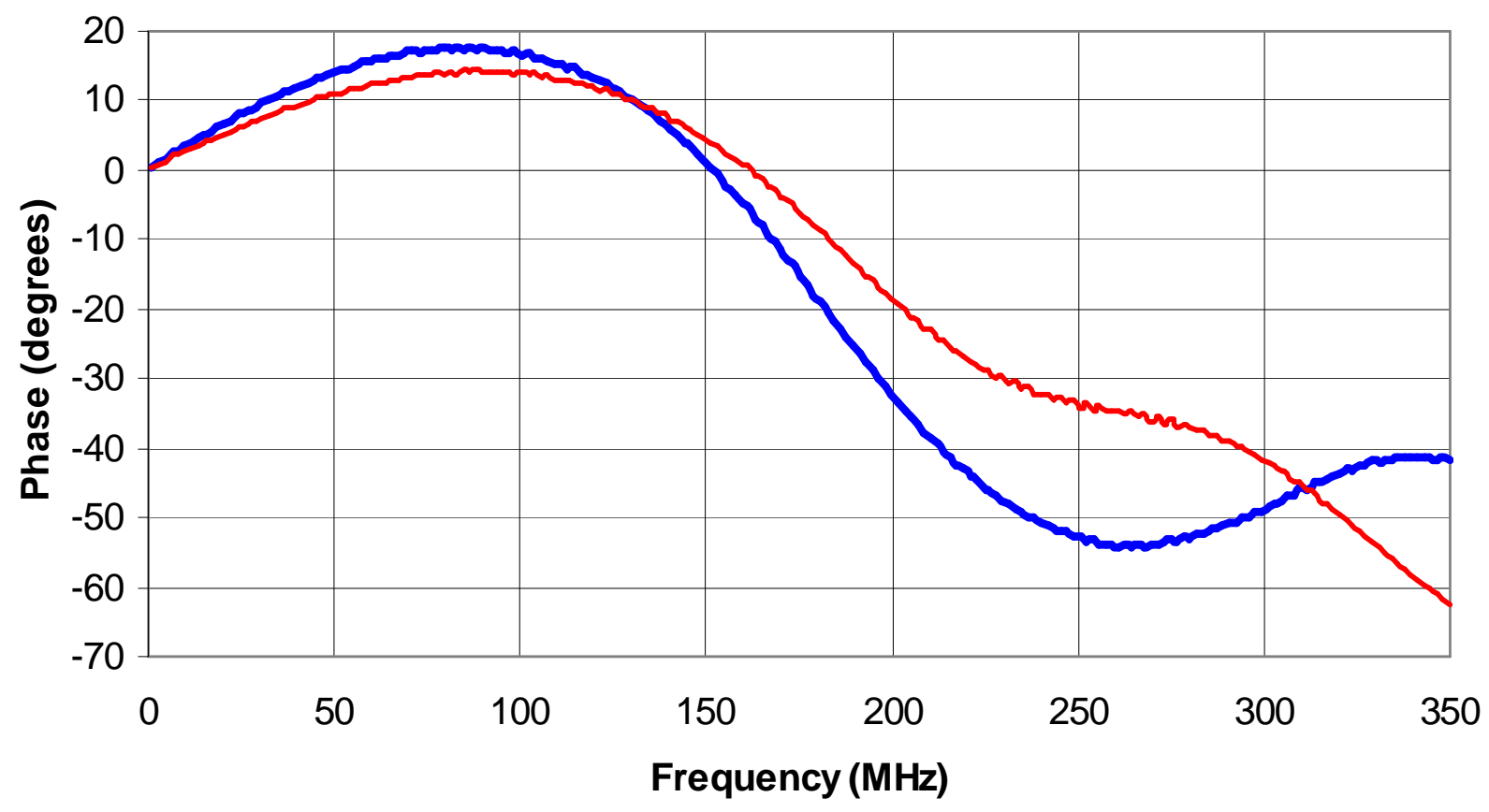

(b)

Figure 3.14 - Magnitude (a) and phase (b) characteristics of the two $175 \mathrm{MHz}$ transducers. 
The phase characteristics of both devices were also very good with phase angles of $-19^{\circ}$ and $-22^{\circ}$, even easier to drive than the $50 \mathrm{MHz}$ transducers. Observing the reverberations in the traces, the active bandwidth of both these transducers was between 50-160 MHz.

\subsubsection{175 MHz Transducers}

The magnitudes and phases for the $175 \mathrm{MHz}$ V3965 transducers are shown in Figure 3.14 for 1-350 MHz. Observing the magnitudes, the first device in blue follows a bell shaped curve whereas the second device in red deviates from this shape at higher frequencies. At DC the magnitudes were 18 and $19 \mathrm{ohms}$, increasing until $175 \mathrm{MHz}$ with magnitudes at this frequency of 45 and $40 \mathrm{ohms}$. These were reverberant out to $315 \mathrm{MHz}$. The phases increase from $0^{\circ}$ at DC and are inductive until $150 \mathrm{MHz}$ where they became capacitive. The transducers therefore contained an additional inductive component for improved transducer performance, and as a result the phases at $175 \mathrm{MHz}$ are just $-16^{\circ}$ and $-6^{\circ}$. By adding resistance and inductance these become well matched to the $50 \mathrm{ohm}$ electronics, and resistive over a wide range making them the easiest transducers to drive.

This compensation was important, as at $175 \mathrm{MHz}$ the absorption in water is exceptionally high. In addition, the very high transducer frequencies require very thin active elements (element thickness is equal to half the operating wavelength) resulting in limited driving power. The thinner active element causes a larger transducer capacitance, which when combined with the high frequencies results in low transducer impedance, causing large capacitive currents in the device. The thin elements cannot tolerate these high currents with out causing permanent damage, requiring reduced signal power. Panametrics recommend a driving voltage of no more than $300 \mathrm{~V}$ for a $10 \mathrm{MHz}$ device, and no more than $150 \mathrm{~V}$ for a $20 \mathrm{MHz}$ device. This halving of the signal voltage continues for every doubling of the transducer centre frequency from the quoted values, resulting in very low driving voltages for the devices used.

\subsubsection{Transducer Beam Properties}

\subsubsection{Focal Lengths and Element Diameters}

The transducer spherical silica glass lens focuses the ultrasound beam. Focussing concentrates the ultrasound energy to a narrow region of uniform pressure some distance from the device. Figure 3.15 shows a focussed beam with the properties of beam diameter and focal zone $\left(\mathrm{F}_{\mathrm{Z}}\right)$ 
highlighted. These two properties are described below. The focal length is the distance to the centre of the focal zone. A long focal length causes significant amounts of attenuation due to absorption in water (see section 2.5.2).

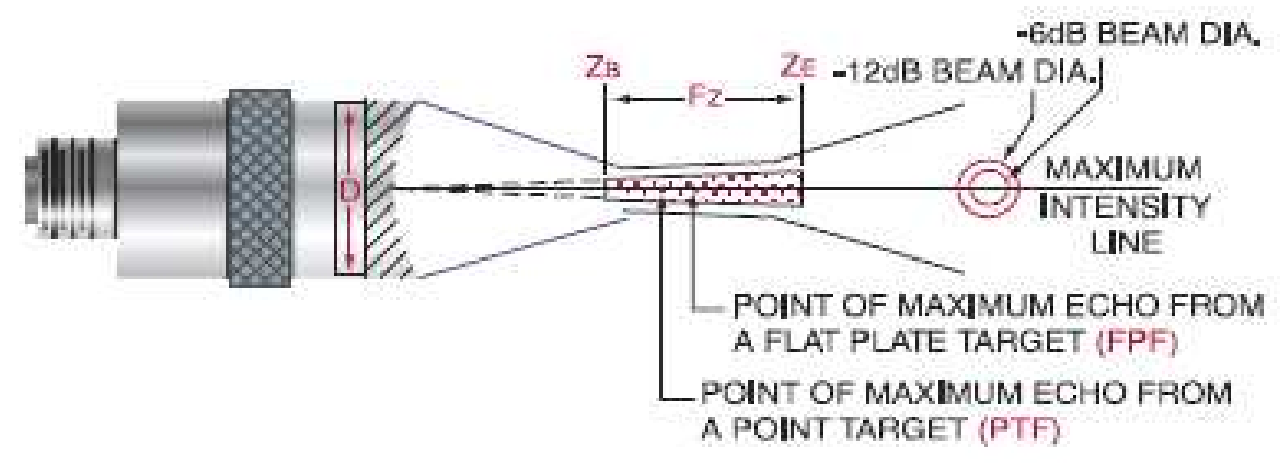

Figure 3.15 - Beam diameter, focal length, and focal zone properties of a transducer [77].

The element diameter and focal length of a transducer determines the beam diameter, near field length, and focal zone of the acoustic beam. Increasing the element diameter narrows the beam diameter and increases the focal zone. Focal lengths and element diameters for the transducers used are shown in Table 3.1, obtained from the manufacturers website [85].

Table 3.1 - Element diameters and focal lengths of immersion transducers used in this work.

\begin{tabular}{llll}
\hline Part Number & $\begin{array}{l}\text { Centre Frequency } \\
(\mathrm{MHz})\end{array}$ & $\begin{array}{l}\text { Focal Length } \\
(\mathrm{mm})\end{array}$ & $\begin{array}{l}\text { Element Diameter } \\
(\mathrm{mm})\end{array}$ \\
\hline V390 & 50 & 13 & 6 \\
V3802 & 110 & 5.8 & 1.8 \\
V3965 & 175 & 6 & 1.5 \\
\hline
\end{tabular}

\subsubsection{Beam Diameters}

The beam diameter is defined as the width of the beam in the transducer focal zone, and is usually measured as the $-6 \mathrm{~dB}$ diameter as shown in Figure 3.15. This is the distance between two horizontally separated points at the focal length where the amplitude of the beam falls $6 \mathrm{~dB}$ below the amplitude measured along the maximum intensity line. The beam diameter of a 
transducer contributes to the lateral resolution and sensitivity of a system. Lateral resolution is the ability of a system to resolve individual targets horizontally spaced apart. Sensitivity is the amount of energy reflected from a target. By concentrating the ultrasound beam this energy is increased. The $-6 \mathrm{~dB}$ beam diameter $(B D)$ for a focussed device is given by (3.9) from [77].

$$
B D(-6 d B)=1.02 \frac{F v}{f D}
$$

where $F$ is the transducer focal length, $v$ is the material sound velocity, $f$ is the frequency, and $D$ is the element diameter. Assuming a pure water path (given the samples are thin and placed in the focal zone), the material velocity can be assumed to be approximately $1480 \mathrm{~ms}^{-1}$ at room temperature.

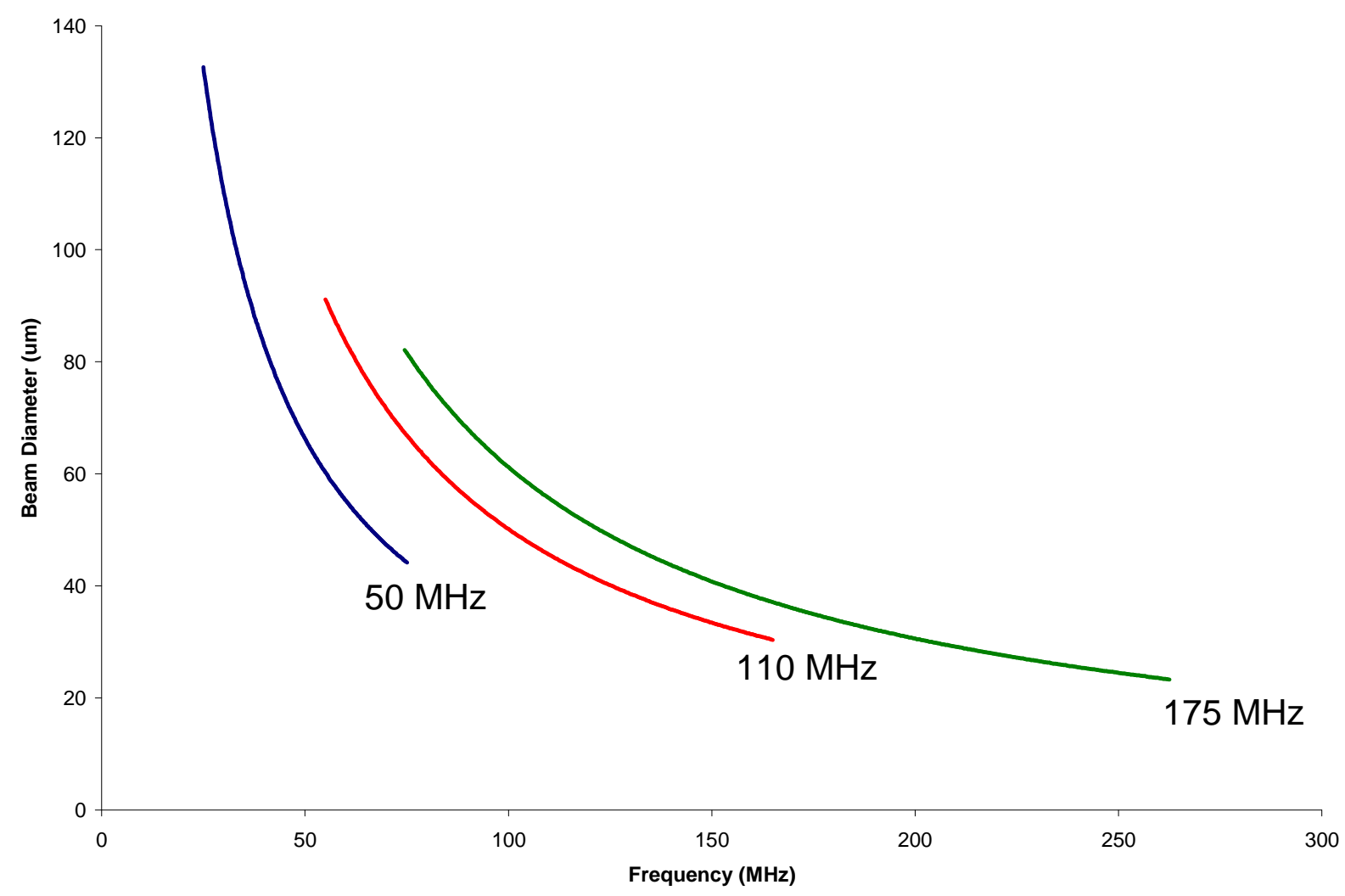

Figure 3.16 - Transducer beam diameter versus frequency for $50 \mathrm{MHz}, 110 \mathrm{MHz}$, and $175 \mathrm{MHz}$ immersion transducers in water. 
Using (3.9) and the focal lengths/element diameters of the transducers, the beam diameter for the three different transducers are plotted in Figure 3.16 over their respective operating bandwidths. For the $50 \mathrm{MHz}$ transducers, diameters from 40-130 $\mu \mathrm{m}$ are observed over the operating range of $25-75 \mathrm{MHz}$. For the $110 \mathrm{MHz}$ transducers, diameters from 30-100 $\mu \mathrm{m}$ are observed over the operating range of $50-160 \mathrm{MHz}$. The $175 \mathrm{MHz}$ transducers show similar diameters to the $110 \mathrm{MHz}$ devices.

\subsubsection{Focal Zones}

The focal zone is the region where the transducer beam applies uniform sound pressure. This is generally chosen to match the thickness of the material to be tested. A reduction in the focal zone increases energy concentration, improving sensitivity, but reducing the radiated area.

The start and end points of a focal zone as shown in Figure 3.15 are defined where the on-axis pulse echo signal amplitude falls to $-6 \mathrm{~dB}$ of the signal amplitude at the focal point. Focal zone is dependent upon the near field $N$ of a transducer and the normalised focal length $S_{\mathrm{F}}$. The near field is the region directly in front of the transducer beam where the ultrasonic amplitude goes through a series of maxima and minima ending at the last maximum. Due to this variation it is normally undesirable to place a target within the near field, instead it is placed at the final maximum. Equations (3.10) and (3.11) show the equations for the near field and normalised focal lengths of a transducer respectively [77].

$$
\begin{aligned}
& N=\frac{D^{2} f}{4 v} \\
& S_{F}=\frac{F}{N}
\end{aligned}
$$

Once the near field and normalised focal lengths have been determined at the appropriate frequencies, the focal zone can be calculated using (3.12) as obtained from [77].

$$
F_{Z}=N S_{F}^{2}\left(\frac{2}{1+0.5 S_{F}}\right)
$$




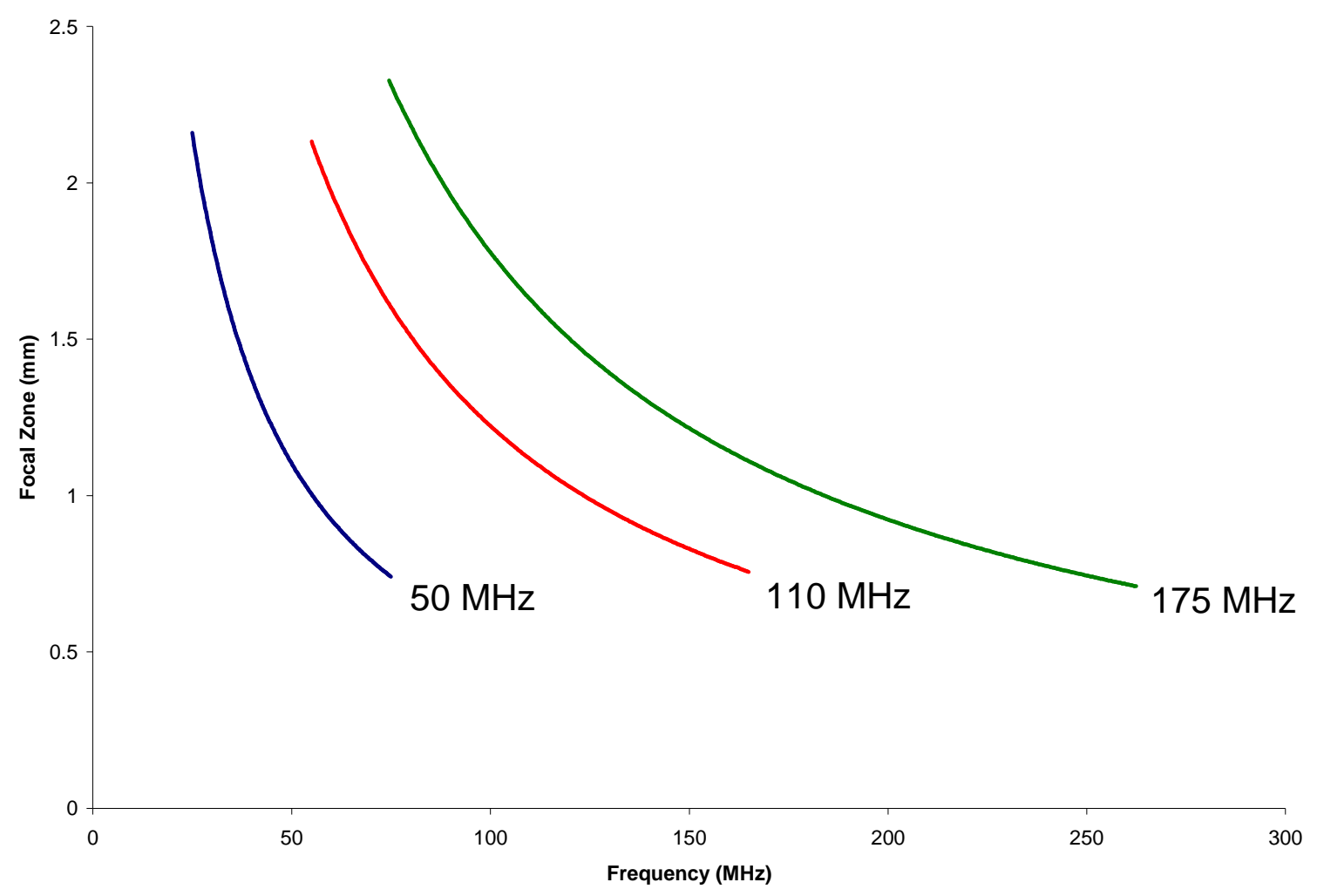

Figure 3.17 - Focal zone length with frequency for the $50 \mathrm{MHz}, 110 \mathrm{MHz}$, and $175 \mathrm{MHz}$ immersion transducers in water.

Using the expression for the focal zone given by (3.12), the frequency dependence of the transducer focal zones are plotted in Figure 3.17. From this it can be observed the focal zones of all three transducers are approximately the same, between 0.75-2 $\mathrm{mm}$ across their respective operating bandwidths. Like the beam diameters, these focal zones of 0.75-2 $\mathrm{mm}$ are an excellent trade off between sensitivity and ease of use.

\subsection{Development of High Frequency Electronics}

As mentioned previously, the high frequencies used in this work cause significant ultrasonic signal attenuation and limit the transducer driving power. This results in received signals with small amplitudes, comparable to the background noise. To account for the above factors, specialised custom electronics were designed in this work. Transmitter and receiver boards were initially designed and constructed to accommodate the $50 \mathrm{MHz}$ device bandwidths of approximately 20-80 MHz. Later an additional wideband receiver and transmit-receive switch 
were made, and a power amplifier purchased for use with the $110 \mathrm{MHz}$ and $175 \mathrm{MHz}$ transducers.

\subsubsection{Board Layout Considerations}

The high frequencies and large bandwidths of the transducer electronics required care in PCB layouts to ensure good circuit performance. Feedback resistors were placed close to the inverting terminals of op-amps, and the ground plane copper was removed directly underneath all inverting inputs; both of these methods were used to minimise stray capacitance. A ground plane was used for all circuits to prevent large area signal return paths to reduce inductance and prevent interference from external fields; minimal cuts were made in the ground plane to ensure this. Analogue amplifying circuitry was separated from the DC and digital control circuitry so that noise generated by fast switching devices did not contaminate the small signals being amplified. Significant bypassing of all supply rails was implemented. All amplifiers in the circuits that were bandwidth critical were current feedback devices with very fast slew rates and large gain bandwidths. Small valued resistors were used to minimize Johnson noise of the circuits, and also preserve the bandwidths of the current feedback amplifiers that are determined by the value of the feedback resistors. All resistors and capacitors were low inductance type and as small as possible to minimize physical board size.

\subsection{2 $100 \mathrm{MHz}$ Transmitter}

The transmitter circuit drove the transmitting transducer with the required waveform, delivering higher power than the function generator was capable of providing. The transmitter schematic is shown in Figure 3.18 (for a full circuit diagram of the designed circuit with component values, power supplies etc., see appendix A1). The power delivered to the transducer was limited to approximately $1.2 \mathrm{~W}$, with a theoretical gain of approximately $13 \mathrm{~dB}$, well within the power dissipation limits of the transducer. The transmitter was designed to operate with a bandwidth of $100 \mathrm{MHz}$ to cover the $50 \mathrm{MHz}$ transducer bandwidth. The circuit output drives the transducer via a 2:1 output transformer to better match the $30 \mathrm{ohm}$ impedance of the transducer (see section 3.2.4.1) to that of the $50 \mathrm{ohm}$ transmitter circuit. The transmitter circuit consists of four amplifiers arranged in a bridge circuit configuration. The amplifiers used were the THS3092 from Texas Instruments as they are dual high speed, broadband, current feedback operational amplifiers. 


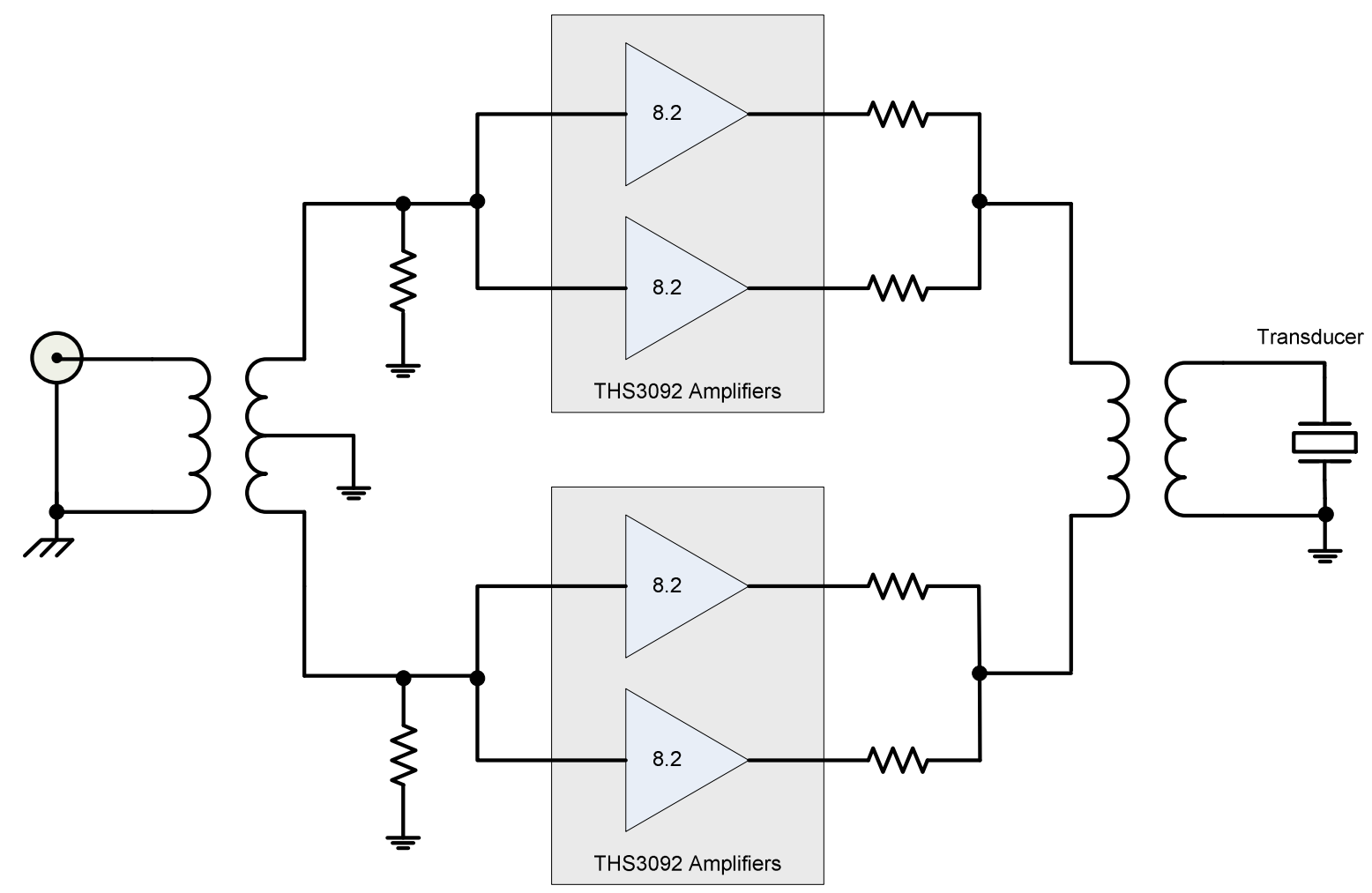

Figure 3.18 - Transmitter schematic.

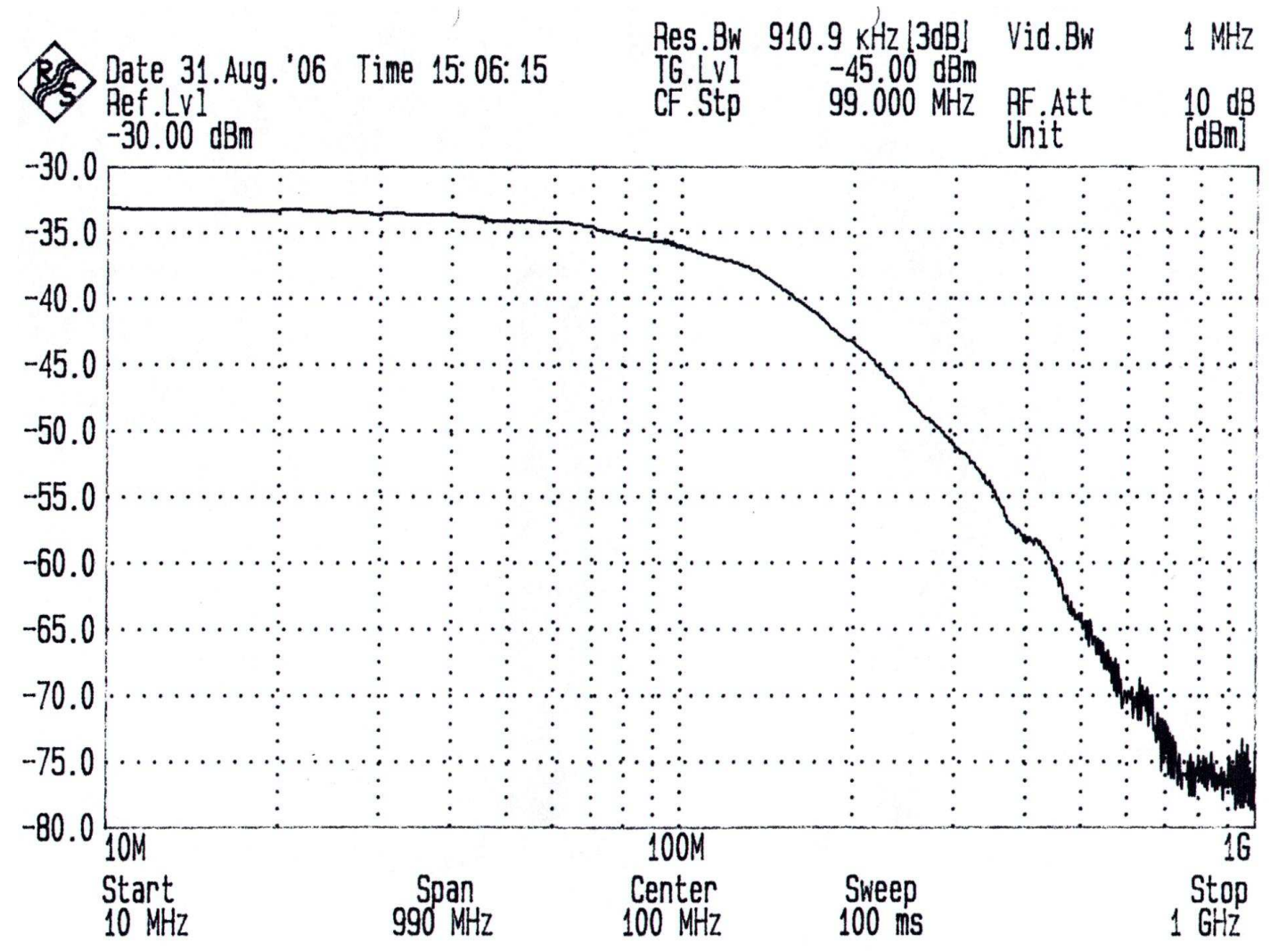

Figure 3.19 - Transmitter gain versus frequency. 
The circuit takes a burst of amplitude $2.5 \mathrm{~V}$ and amplifies this by a gain of approximately 4 to just over $10 \mathrm{~V}$. The input transformer provides a balanced input and isolation from the function generator earth. The input is matched to the excitation impedance via matching resistors. The input transformer creates the input signal and its inverse on the secondary windings, both of which are amplified independently using separate amplifier paths. Paralleling the op-amps on the output increases the current drive of the circuit capable of delivering a peak output current of $1 \mathrm{~A}$. The amplified signals are then combined through the output transformer to drive the transmitting transducer. Figure 3.19 shows the gain bandwidth characteristics of the circuit. With a source power of $-45 \mathrm{dBm}$, a power gain of $12 \mathrm{~dB}$ is observed and a $-3 \mathrm{~dB}$ bandwidth of $100 \mathrm{MHz}$ achieved. This accommodates the broad bandwidth of the $50 \mathrm{MHz}$ transducers.

\subsection{3 $100 \mathrm{MHz}$ Receiver}

The receiver circuit formed the interface between the receiving transducer and the oscilloscope and served to amplify the small signals before capture by the oscilloscope. Significant water absorption and sample scattering combined with low transmit transducer power meant that receiver noise performance was critical to discriminate signals from background noise. To accommodate different signal strengths due to variable sample scattering properties, the receiver gain needed to be variable over a large range and possess a flat gain response over the transducer bandwidth. Finally, signals received later in time incur greater attenuation than those received earlier, having travelled a greater distance. To compensate time gain compensation was added as a feature in the event it was required. This receiver was designed to accommodate the $50 \mathrm{MHz}$ transducers only.

\subsubsection{Circuit Operation}

The schematic of the receiver is shown in Figure 3.20 (for a full circuit diagram of the designed circuit with component values, power supplies etc., see appendix A2). The input impedance of the amplifier was $50 \mathrm{ohm}$. Two AD8337 variable gain amplifiers (VGAs) from Analog Devices were connected in series to form the front end of the circuit. These VGAs possessed very low current and voltage noise characteristics $(4.8 \mathrm{pA} / \mathrm{rt} \mathrm{Hz}$ and $2.2 \mathrm{nV} / \mathrm{rt} \mathrm{Hz})$, wide bandwidth, fast slew rates, large variable gains, and accommodate a linear-in-dB gain control method. This gain control was useful as by ramping the voltage applied to the gain control pin, linear-in-dB time gain compensation could be achieved. 


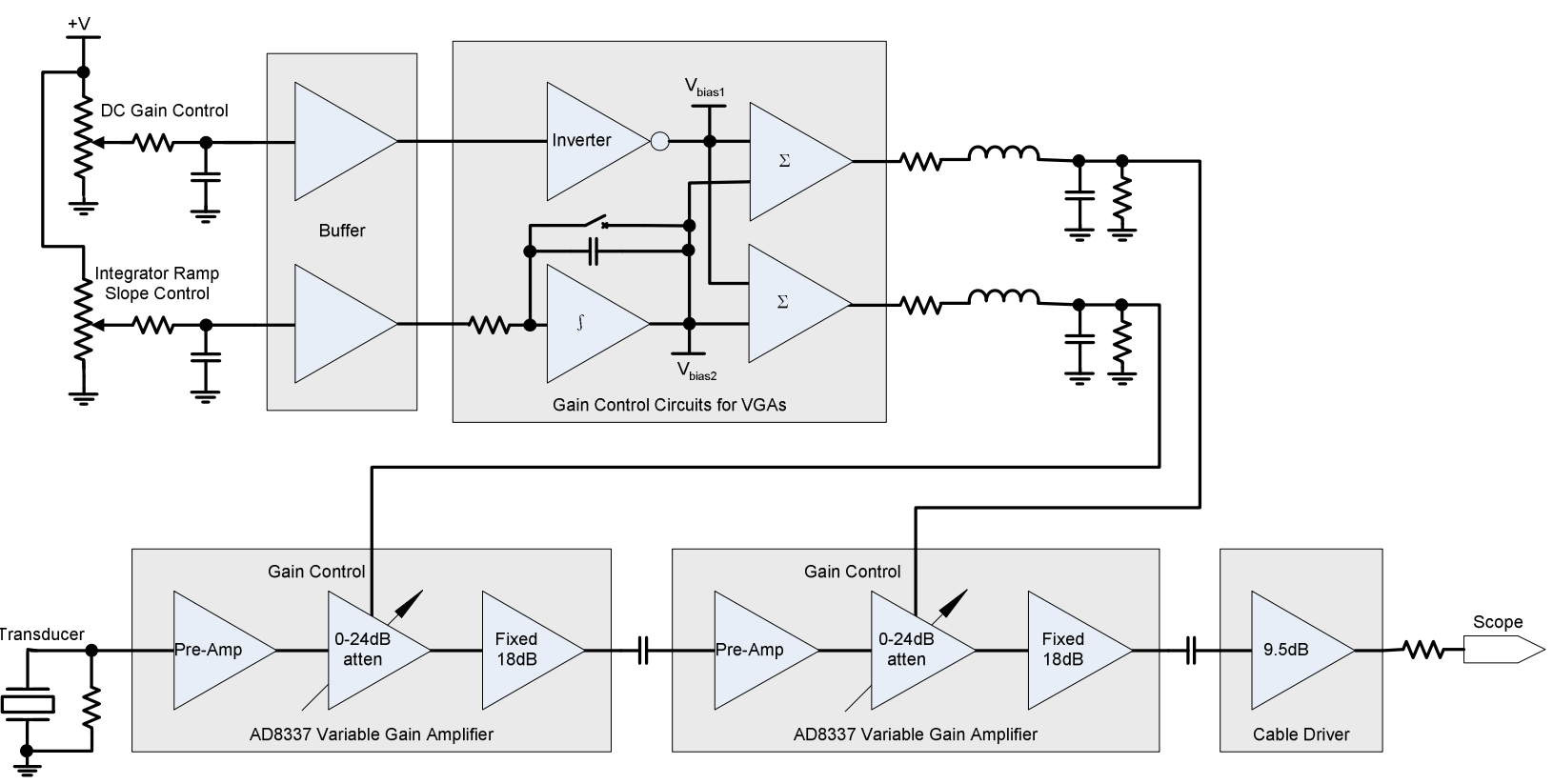

Figure $3.20-100 \mathrm{MHz}$ receiver schematic.

This compensates for the attenuation caused by propagation within a material, applying greater compensation to signals arriving later in time having travelled a greater distance. The time gain compensation method is described in more detail in the next section. A user adjustable DC input to an op-amp integrator [87], with an analogue switch for integrator reset generates the ramp for this function. The op-amp used was a OPA2690 from Texas Instruments and was selected for its fast rise and settling time characteristics in order to achieve the best ramp performance. The integrator is shown in Figure 3.20 as an amplifier block with an integral symbol with the discharge switch in the op-amp feedback path. The integrator slope control potentiometer determines the slope of the integrator ramp - the degree of attenuation compensation. The function generator SYNC signal activates the integrator reset, discharging the capacitor, and resetting the time gain compensation to zero.

The VGAs consisted of a configurable pre-amp, typically set for a gain of 3 , followed by a $24 \mathrm{~dB}$ attenuator, and a fixed $18 \mathrm{~dB}$ amplifier. The output amplifier that drives the transmission cable to the oscilloscope was configured for a gain of 3. This results in a variable gain from 17-65 dB. The summing blocks seen in Figure 3.20 provided the gain control interface to the two VGAs. These summing blocks controlled the time gain compensation and DC gain requirement taking inputs from both potentiometers. These are configured such that once the 
first VGA reaches its maximum gain, the second VGA gain begins to increase. Using this method both amplifiers can be dedicated solely to DC gain, time-gain compensation, or any combination of the two. The summing blocks were implemented using op-amps (OPA2690 ICs) configured as summing junctions. The control voltages from the summing junctions were low pass filtered to ensure stable gain control before being sent to the VGA. AC coupling was used along the amplifier chain to prevent DC offsets.

\subsubsection{Time Gain Compensation Method}

To account for attenuation of the ultrasound with depth electronically, an expression for the VGA gain in $\mathrm{dBmm}^{-1}$ as a function of the user adjustable input voltage must be defined. The variation in VGA gain with gain pin voltage was obtained from the AD8337 datasheet given by

$$
\operatorname{Gain}_{V G A}(d B)=19.7 V_{\text {Gain }}+12.65
$$

where $\operatorname{Gain}_{\mathrm{VGA}}(\mathrm{dB})$ is the VGA gain in $\mathrm{dB}$ and $V_{\mathrm{Gain}}$ is the voltage applied to the VGA gain pin. As described in the previous section, an op-amp integrator generated a ramp that was applied to the VGA gain pin to achieve linear-in-dB gain control. The output of an op-amp integrator is given by equation (3.14) [87]

$$
V_{\text {out }}=-\frac{1}{R C} \int_{0}^{T} V_{\text {in }} d t
$$

where $V_{\text {in }}$ is the user adjustable input voltage to the op-amp integrator, $R$ is the integrating resistor, $C$ the integrating capacitor, and $T$ is the duration of the ramp. For a DC input voltage the output is then given by $(3.15)$

$$
V_{\text {out }}=-\frac{T}{R C} V_{\text {in }}
$$

Assuming no DC gain, the output from the integrator ramp is equal to the VGA gain pin voltage. Substituting $V_{\text {out }}$ for $V_{\text {Gain }}$ in equation (3.13) and inverting the integrator output (eliminating the negative sign) the expression for VGA gain becomes 


$$
\operatorname{Gain}_{V G A}(d B)=19.7 \frac{T}{R C} V_{i n}+12.65
$$

Since attenuation is expressed in $\mathrm{dBmm}^{-1}$, equation (3.16) is divided through by $x$ representing the distance travelled through a material. Substituting $x=v t$ where $t$ is the time of flight, and $v$ is the bulk velocity in the sample (here a velocity for water of $1500 \mathrm{~ms}^{-1}$ has been assumed), the final expression for VGA gain in $\mathrm{dBmm}^{-1}$ is given by (3.17)

$$
\operatorname{Gain}_{V G A}\left(\mathrm{dBmm}^{-1}\right)=\frac{13.1 \times 10^{-6}}{R C} V_{\text {in }}+8.4 \times 10^{-6}
$$

By fixing $R$ and $C$ at appropriate values, the degree of time compensation is varied by the user adjustable integrator DC input.

\subsubsection{Circuit Performance}

The receiver circuit frequency response from minimum to maximum gain is shown in Figure 3.21 for five different gains. The top trace is maximum gain and the bottom trace is minimum gain. The source power of the spectrum analyser was $-30 \mathrm{dBm}$ with $20 \mathrm{~dB}$ of external attenuation to limit the receiver output signals at maximum gain to less than the supply rails. Each of the traces displays excellent frequency response, satisfying the transducer requirements with a steep roll off at higher frequencies. The lowest trace is observed to have a magnitude close to $-32 \mathrm{dBm}$. This agrees with that expected as $-50 \mathrm{dBm}$ plus minimum gain $(17 \mathrm{~dB}$ ).equals $-33 \mathrm{dBm}$. Using the same method for maximum gain of $65 \mathrm{~dB}$ results in 15 $\mathrm{dBm}$, also in agreement with Figure 3.21.

Figure 3.22 plots the noise of the receiver and the spectrum analyser used to perform the measurement. The lower trace was the analyser noise alone, while the higher trace was the measured noise from the output of the receiver circuit when configured for maximum gain. For this trace the analyser was measuring solely the output noise of the receiver at maximum gain plus the intrinsic analyser noise. 


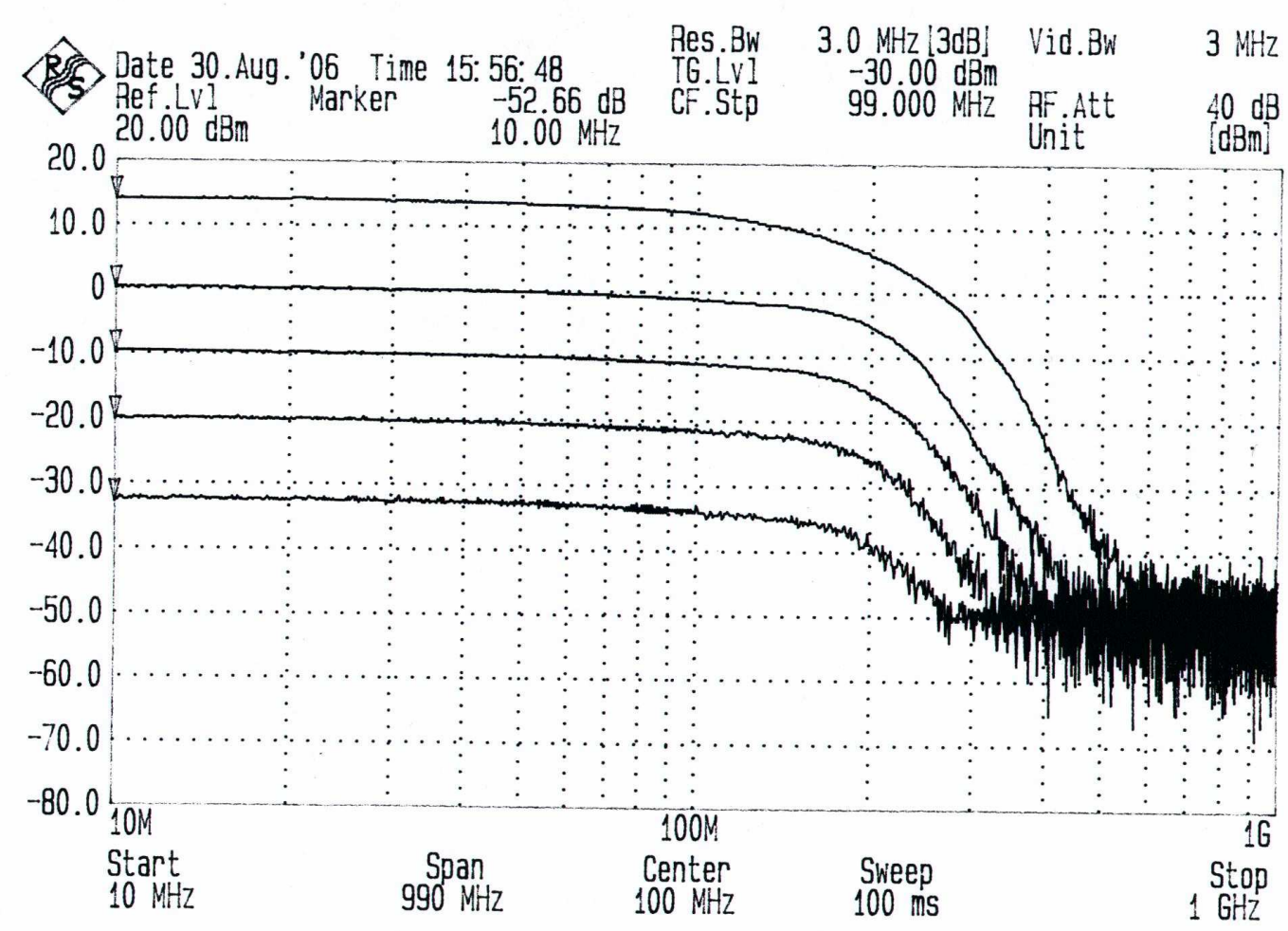

Figure 3.21 - Receiver gain versus frequency for five different gains from minimum gain of $17 \mathrm{~dB}$ (lowest trace) to maximum gain of $65 \mathrm{~dB}$ (highest trace).

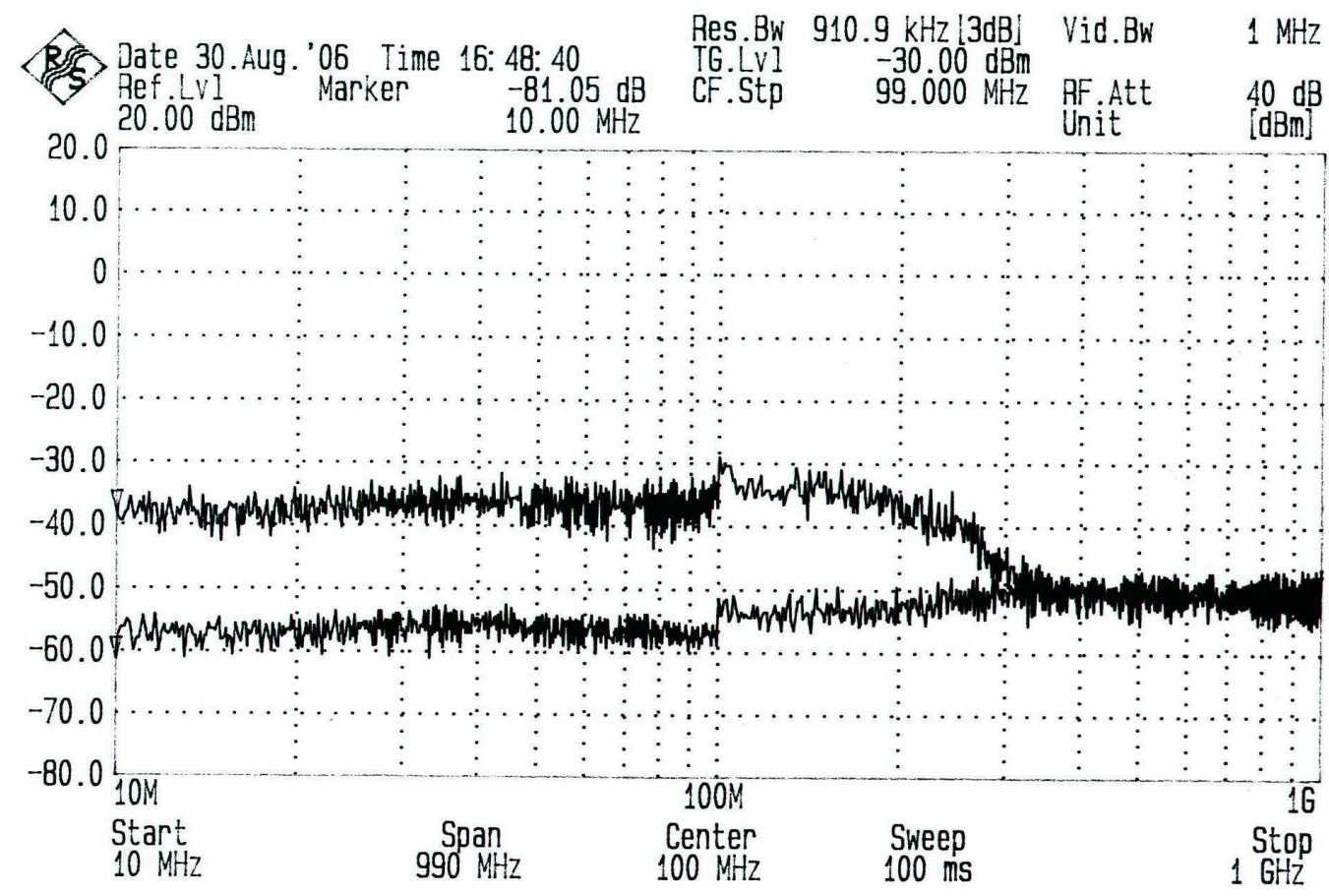

Figure 3.22 - Receiver output noise (top) and spectrum analyser noise (bottom) traces. 
Without maximum receiver gain the receiver noise could not be distinguished from that generated by the analyser. The sudden step in noise on both signal traces above $100 \mathrm{MHz}$ was a property of the analyser as it changed scales internally. The receiver output noise was calculated by subtracting the spectrum analyser noise from the top trace. This value was then divided by the square root of the analyser resolution bandwidth (here $910.9 \mathrm{kHz}$ ) expressing the noise in the standard form of $\mathrm{nV} / \sqrt{\mathrm{Hz}}$ (nanovolts per root hertz). Finally, dividing through by the maximum gain of the receiver of $65 \mathrm{~dB}$ equates to an input referred noise value of approximately $2 \mathrm{nV} / \sqrt{\mathrm{Hz}}$, equal to the AD8337 input referred voltage noise from the amplifier datasheet. This indicated the circuit noise was dominated by the noise of the first VGA.

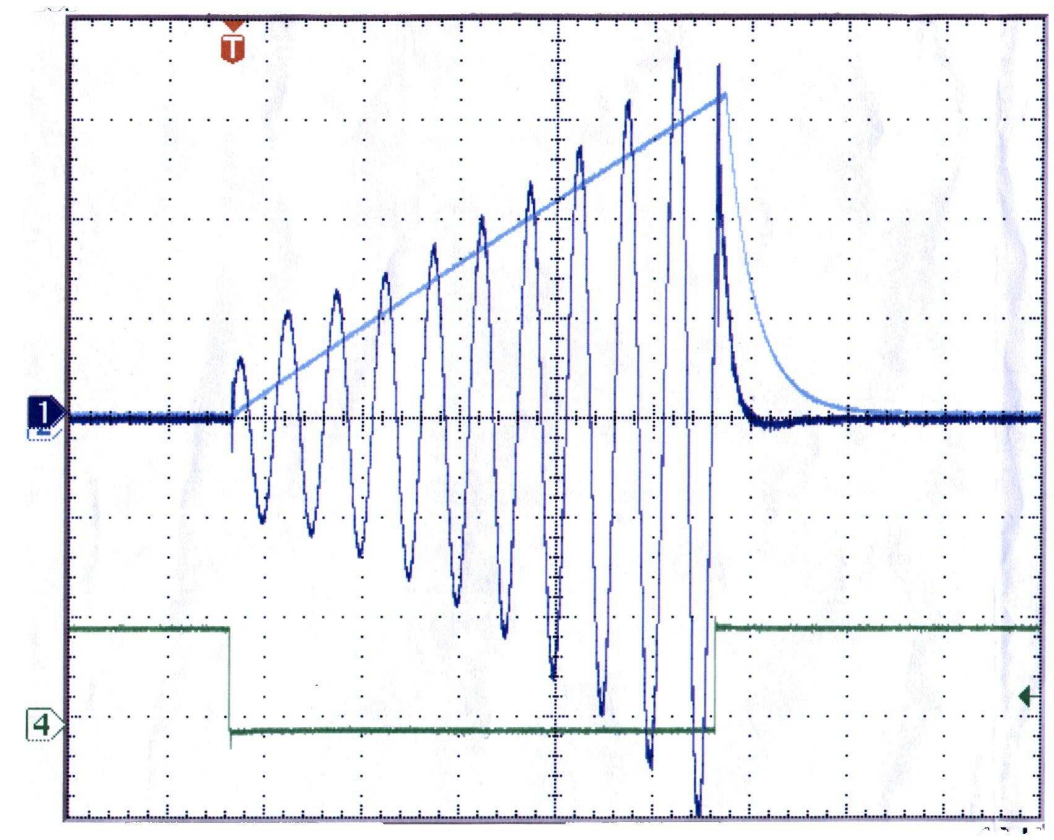

Figure 3.23 - A time gain compensated waveform shows an exponential rise, with the square wave SYNC signal resetting the integrator ramp to zero, and thus stopping the time gain compensation.

A time gain compensated waveform is displayed in Figure 3.23 with associated control signals. This shows an exponentially growing waveform to counter the sample attenuation. The integrator ramp implementing the VGA gain control is shown along with the function generator SYNC signal setting the start and finish of the time gain compensation period. The SYNC signal goes low once the waveform is generated. This charges the integrating capacitor, activating the integrator, generating the VGA gain control ramp. Once the waveform ends the SYNC signal goes high, activating the integrator capacitor discharge switch, deactivating and resetting the ramp, stopping the time gain compensation until the start of the next waveform. 


\subsubsection{MHz Minicircuits 20W Power Amplifier}

During the project it was decided to also use $100 \mathrm{MHz}$ and $175 \mathrm{MHz}$ transducers in addition to the initial $50 \mathrm{MHz}$ transducers. To design another transmitter with a bandwidth to accommodate $200 \mathrm{MHz}$ waveforms and their higher frequency components would take a considerable amount of design so a commercial power amplifier was purchased. The unit purchased was a 20 W ZHL-20W-13 from Minicircuits, possessing a bandwidth of $10-1000 \mathrm{MHz}$ and a gain of $50 \mathrm{~dB}$. It was matched for $50 \mathrm{ohm}$ input and output impedances, and could withstand a full impedance mismatch (short or open) across all phases at the output, assuming the maximum output power was never exceeded.

\subsection{5 $500 \mathrm{MHz}$ Receiver}

To accommodate the use of higher frequency transducers, an additional receiver circuit was designed to accommodate the frequency range of all three transducers covering 20-500 MHz. Very low noise and a large gain were the design considerations. This circuit design did not include time gain compensation as it was found this feature was not required often enough when using the $100 \mathrm{MHz}$ receiver. Variable gain was also not included as the use of the oscilloscope gain was deemed sufficient. The receiver schematic is shown in Figure 3.24 (for a full circuit diagram of the designed circuit with component values, power supplies etc., see appendix A3).

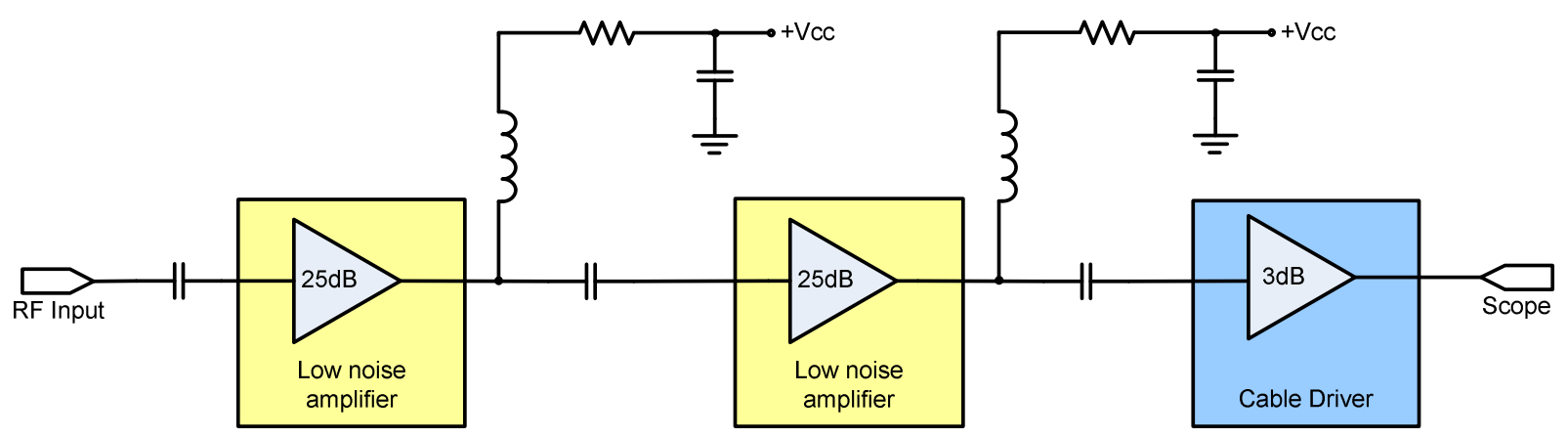

Figure 3.24 - $500 \mathrm{MHz}$ receiver schematic. 


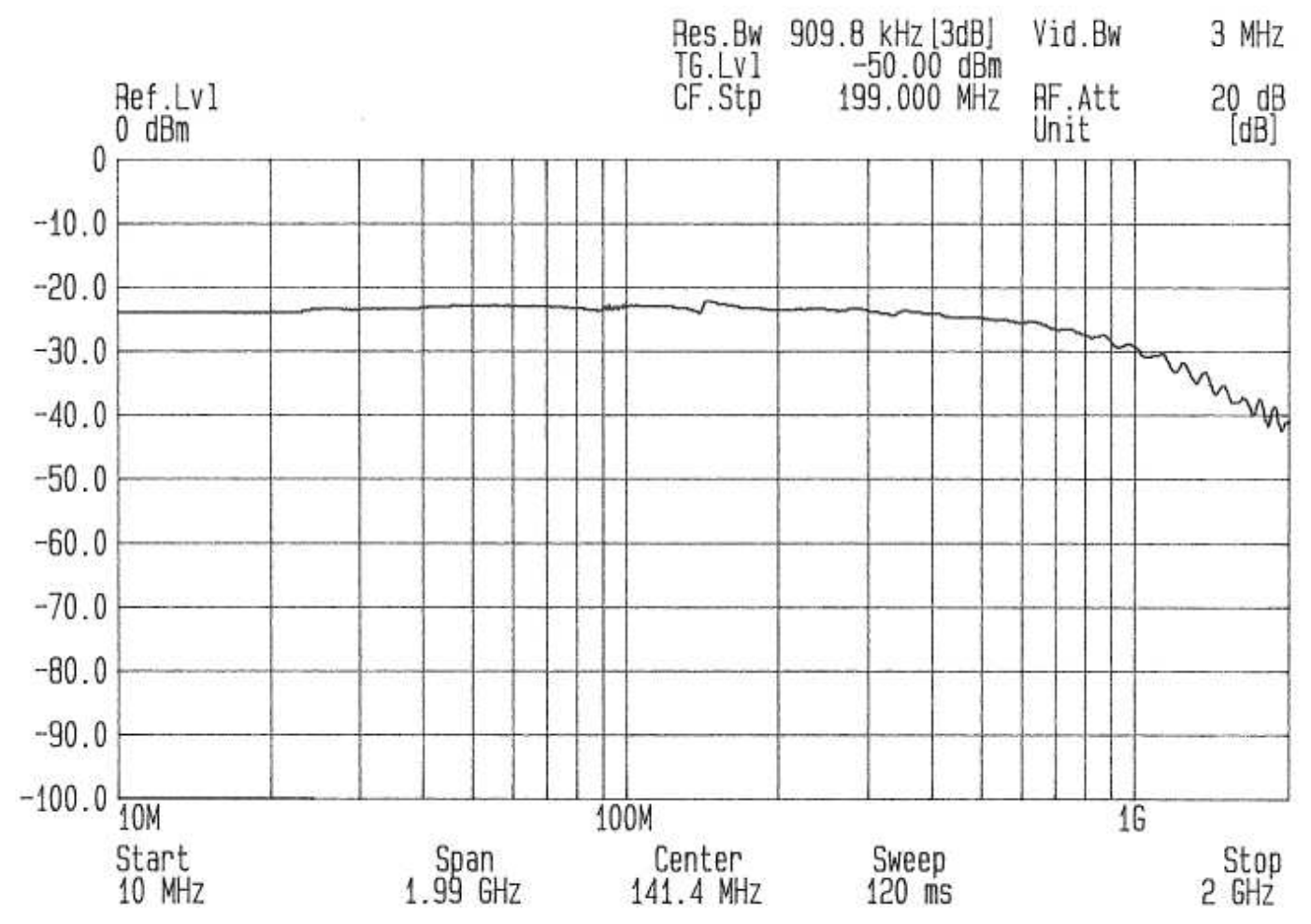

Figure 3.25 - Receiver gain versus frequency.

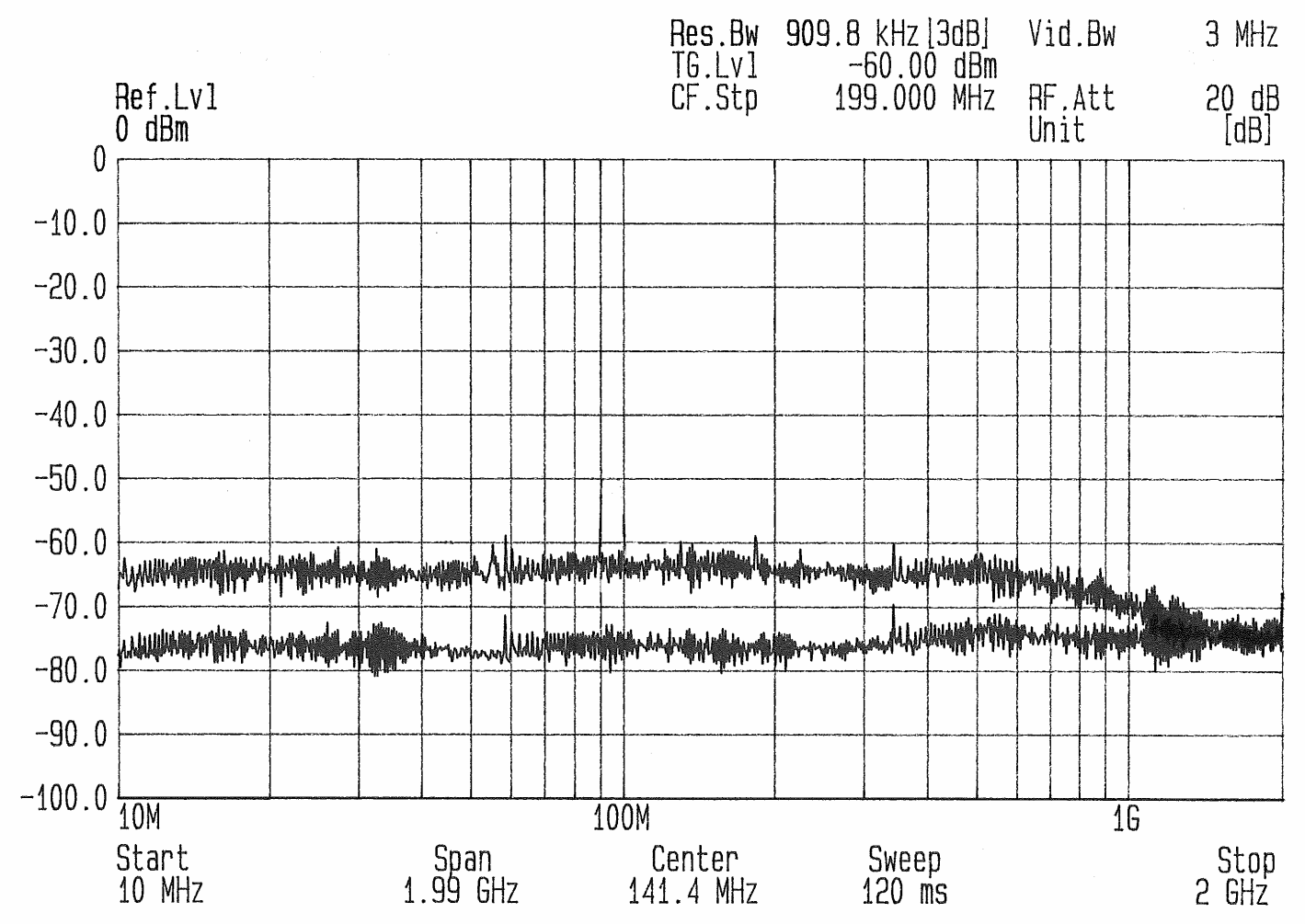

Figure 3.26 - Receiver circuit and spectrum analyser noise traces. 
Two low noise amplifiers connected in series perform the amplifying of the circuit. The low noise amplifiers are Gali-74+ amplifiers from Minicircuits that possess a bandwidth extending from DC to $1 \mathrm{GHz}$ with a fixed gain of $25.1 \mathrm{~dB}$ and a maximum power output of $19.2 \mathrm{dBm}$. They were matched to $50 \mathrm{ohm}$ input and output impedances and were unconditionally stable. This is an important property since transducer impedance varies with frequency and almost never matches the $50 \mathrm{ohm}$ receiver; they possessed an excellent noise figure of just $2.1 \mathrm{~dB}$. The low noise amplifiers received DC power and transmit AC output through the same terminal and required the use of an appropriate inductor to separate the two. The resistor in the DC biasing arm of the circuit provided the bias current with the value determined by the DC supply voltage. AC coupling is used between all stages to eliminate DC offsets. An optional $6 \mathrm{~dB}$ cable driver can be used to drive the oscilloscope, reducing the load on the amplifiers.

The gain versus frequency characteristic of this receiver is shown in Figure 3.25 and the noise characteristic in Figure 3.26. It shows an overall gain of $47 \mathrm{~dB}$ into a $50 \mathrm{ohm} \mathrm{load,} \mathrm{with} \mathrm{a}$ useable $3 \mathrm{~dB}$ bandwidth exceeding $500 \mathrm{MHz}$. Based on these traces, and following the same noise calculation method from section 3.3.3, the noise performance was calculated to be just $1 \mathrm{nV} / \sqrt{\mathrm{Hz}}$; almost equivalent to the Johnson noise of a $50 \mathrm{ohm}$ resistor.

\subsubsection{MHz Transmit-Receive Switch}

A transmit-receive switch was required when performing a pulse-echo measurement, where a single transducer operates in both transmit and receive mode. As shown in section 3.2, the transducers cover a frequency range of $20-320 \mathrm{MHz}$. Like the $500 \mathrm{MHz}$ receiver circuit, this board was designed for a frequency range of $20-500 \mathrm{MHz}$ to accommodate $200 \mathrm{MHz}$ pulses and their higher frequency components. During the transmit cycle, the transmit-receive switch drives the transducers with a waveform from the power amplifier while simultaneously isolating the receiver during the pulse duration. As the transmitter can generate output waveforms greater than $+20 \mathrm{~V}$, which are well in excess of the receiver circuit inputs, the circuit had to provide enough isolation across all frequencies to prevent receiver damage during transmission. During the receive cycle, the receiver circuit must be sensitive to the ultrasound signals reflected back from the sample whilst isolating the significant output noise from the power amplifier. The schematic of the transmit-receive circuit used is shown in Figure 3.27 (for a full circuit diagram of the designed circuit with component values, power supplies etc., see appendix A4). 


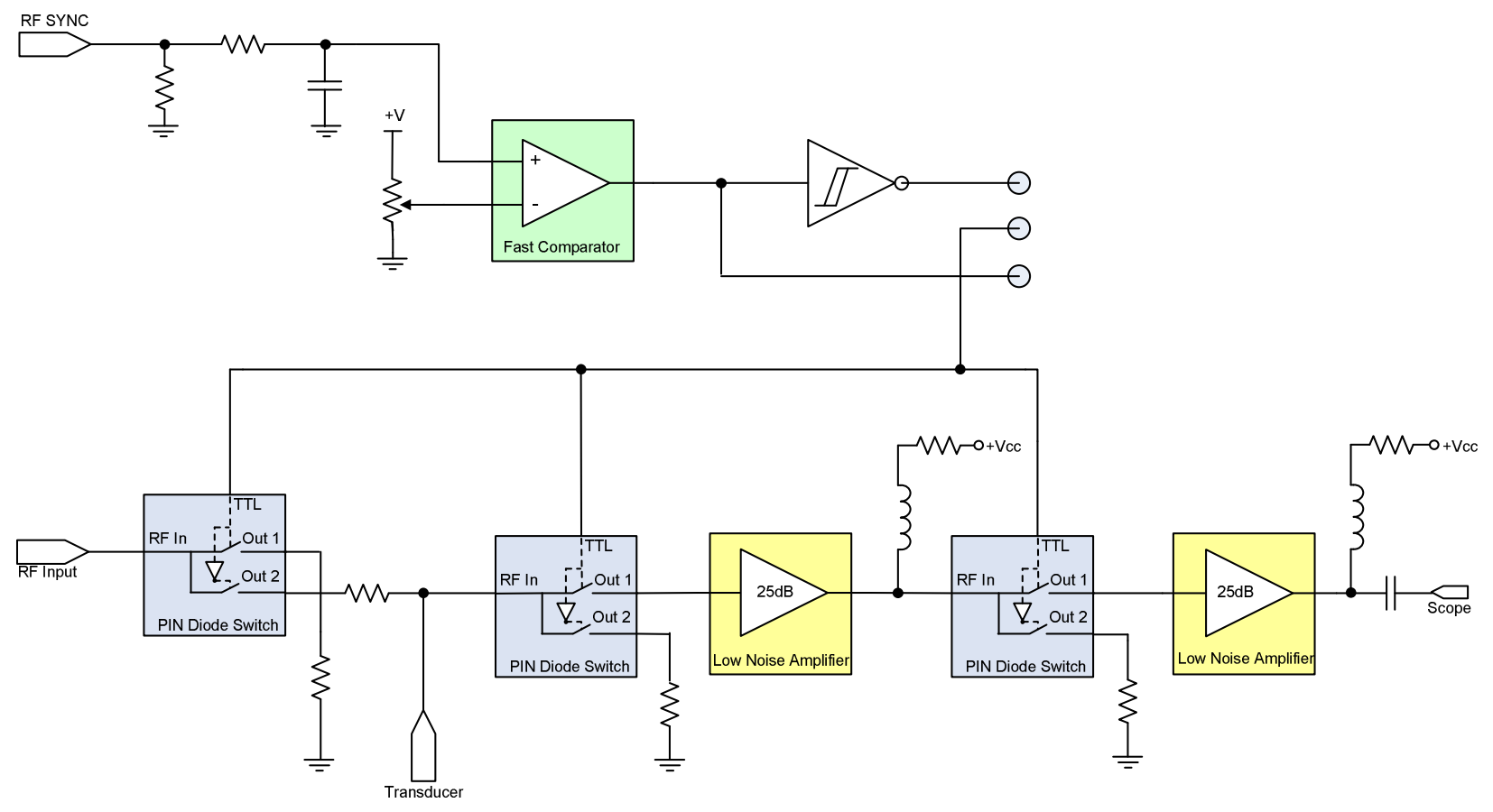

Figure 3.27 - Simplified transmit-receive switch schematic.

The circuit elements used to perform the required switching (shown in purple) are TOSW-230+ single pole double throw PIN diode switches from Minicircuits. PIN diodes were used due to their wide bandwidth and high isolation at very high frequencies. The TOSW-230+ possesses a bandwidth of 10-3000 MHz, low insertion loss, and typical isolation of $40 \mathrm{~dB}$. The PIN diodes are configured as change over switches so when the OUT1 output is on OUT2 is off and viceversa. The input and outputs are matched for $50 \mathrm{ohm}$. The switches are toggled via TTL logic signals with TTL drivers integrated into the TOSW-230+ reducing external circuitry.

Observing Figure 3.27, there are three TOSW-230+ packages in the circuit. The far left switch is used to isolate the power amplifier from the transducer while the other two are used to isolate the receiver circuit. Two switches are used in series to increase the isolation on the receive side due to the large outputs from the power amplifier during transmit. It should be noted the maximum input power into these devices is typically $+30 \mathrm{dBm}$. Even though the driving signals from the power amp greatly exceeded this value, the average power dissipation was kept well below this since the signal duty cycle was no greater than $0.5 \%$.

To avoid the use of an external receiver board, two Gali-74+ low noise amplifiers (LNAs), identical to those used in the $500 \mathrm{MHz}$ receiver circuit (section 3.3.5), were included on board. 
The LNAs were supplied with $+8 \mathrm{~V}$ whilst the rest of the circuit $+5 \mathrm{~V}$. DC blocking capacitors are not required between a TOSW-230+ and Gali-74+ as the PIN diode packages were found to contain internal $1800 \mathrm{pF}$ input and output coupling capacitors.

The function generator SYNC signal controls the PIN diodes and this signal is first passed through an ADCMP601 high-speed comparator, providing a sharp square wave output. The comparator is configured for $20 \mathrm{mV}$ hysteresis and user adjustable reference voltage. The comparator with the adjustable reference voltage ensures an output swing between 0 and $+5 \mathrm{~V}$, independent of the input swing. This signal is then fed directly to a header and an inverted copy to another. A jumper is used to select whether the original or inverted signal is sent to the PIN diodes TTL switch drives. This is so both active high and active low function generator SYNC outputs can be accommodated and is also useful for test purposes.

Figure 3.27 shows for the leftmost PIN diode, the output to the transducer is connected to RF 2. The other two PIN diode outputs are connected to RF 1. This will ensure when the SYNC signal allows the power amplifier to drive the transducer, the receiver PIN diodes are off, isolating the receiver circuit, and vice versa. PIN diode RF outputs not used in the signal path are grounded via $50 \mathrm{ohm}$ resistors ensuring the RF path is always correctly terminated.

\subsubsection{Circuit Performance}

The gain of the receiver circuit versus frequency with the switch in receive mode is shown in Figure 3.28. For an input of $-60 \mathrm{dBm}$, an output power of approximately $-18 \mathrm{dBm}$ is observed at $100 \mathrm{MHz}$, equalling a gain of $42 \mathrm{~dB}$ at $100 \mathrm{MHz}$. Whilst the gain is observed to be several $\mathrm{dB}$ larger for some lower frequencies, $40-42 \mathrm{~dB}$ is the typical gain of the circuit. The gain is relatively flat out to $400 \mathrm{MHz}$ with a $-3 \mathrm{~dB}$ bandwidth of $500 \mathrm{MHz}$.

Theoretically, for the received signal path a $-6 \mathrm{~dB}$ loss is incurred from the $50 \mathrm{ohm}$ voltage divider between the output of the first switch and the input of the first LNA. The signal then undergoes a gain of $25.1 \mathrm{~dB}$ through the LNA followed by another $-6 \mathrm{~dB}$ loss between the second PIN diode and second LNA, followed by a further $25.1 \mathrm{~dB}$ of gain before being received by the $50 \mathrm{ohm}$ scope. This resulted in a theoretical gain of $38.2 \mathrm{~dB}$ with an output of $-21.9 \mathrm{dBm}$ for a $-60 \mathrm{dBm}$ input, in good agreement with that measured experimentally. The extra gain from the circuit is due to slightly increased amplifier bias current. 


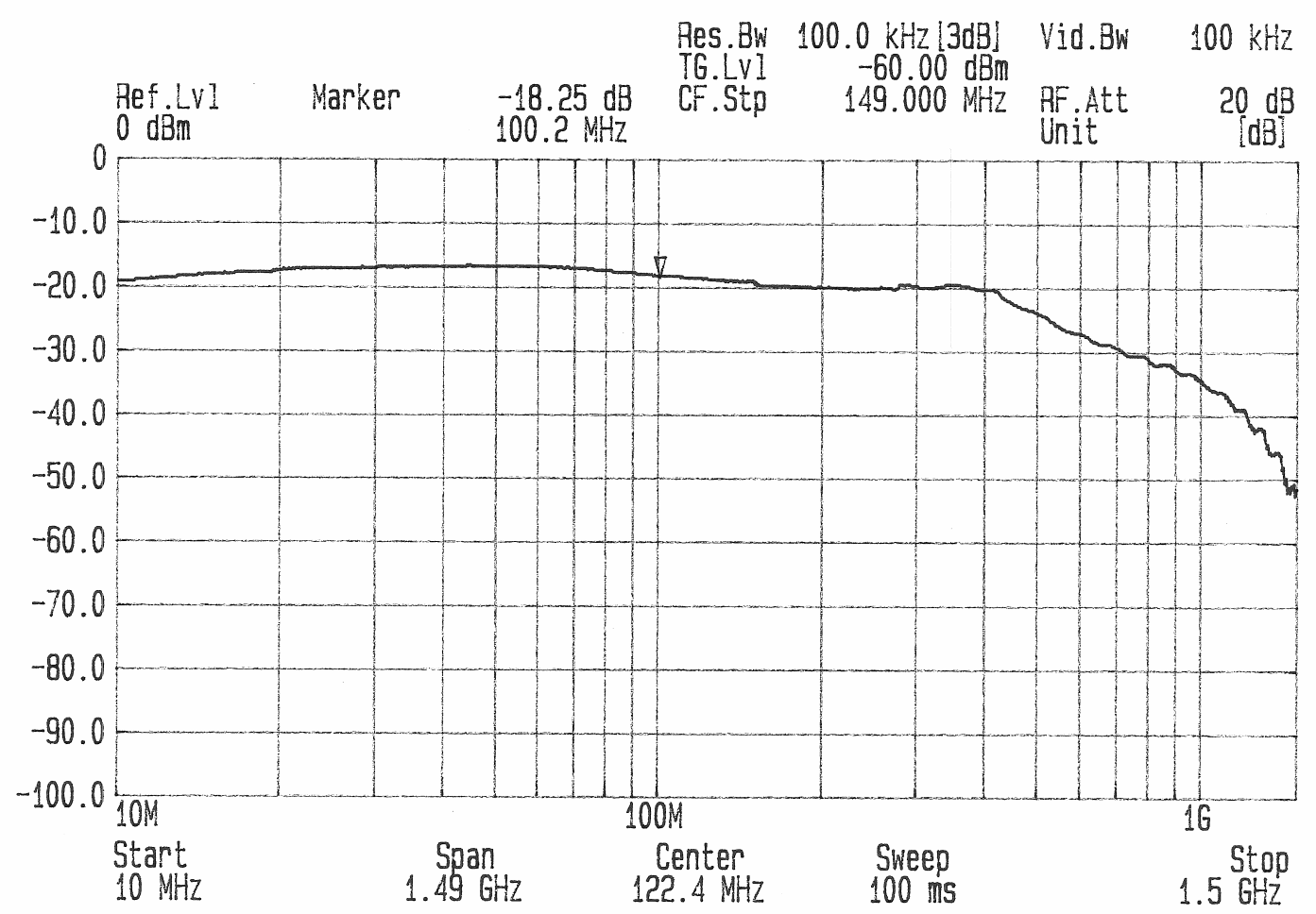

Figure 3.28 - Transmit-receive switch circuit receiver gain with frequency.

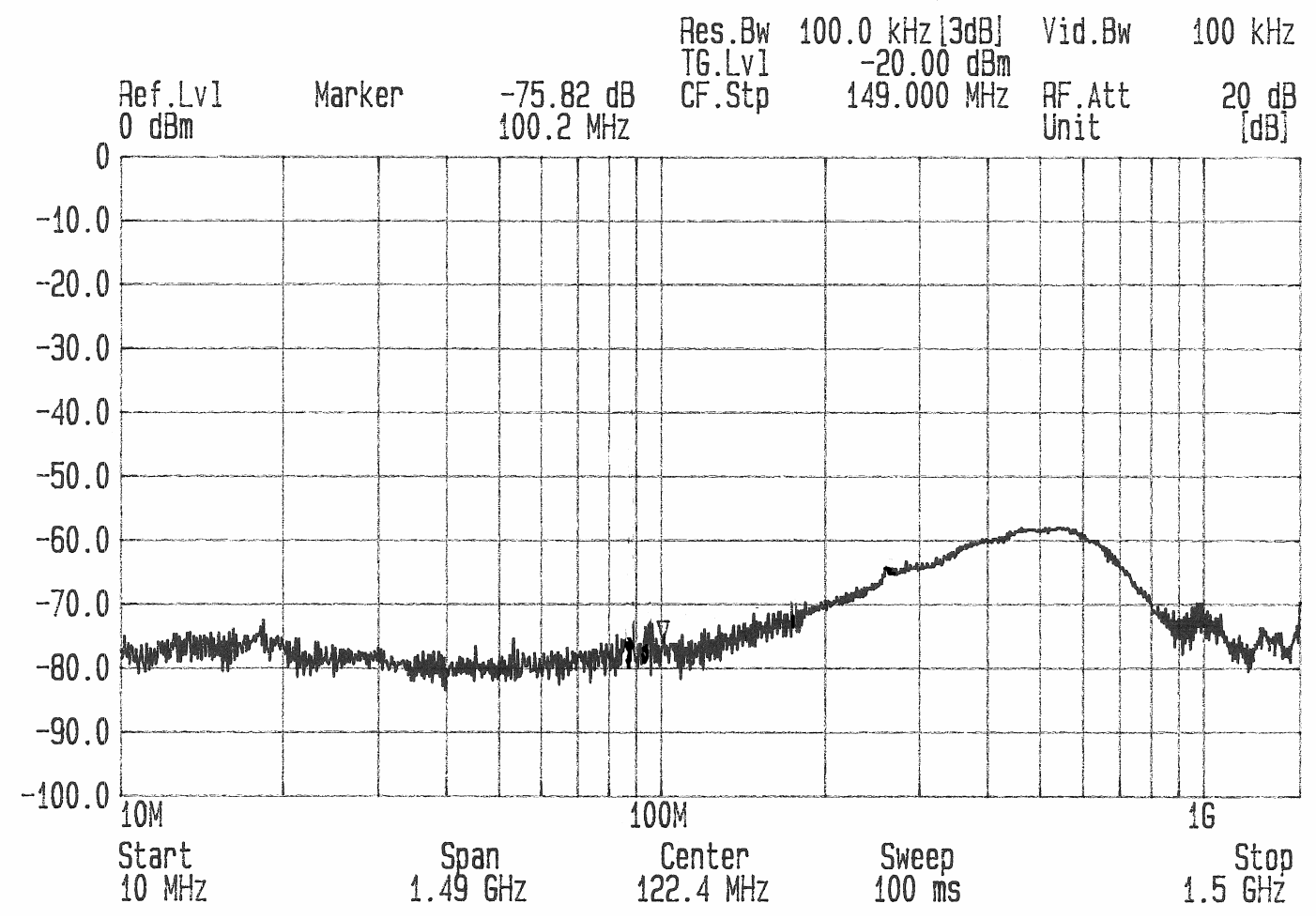

Figure 3.29 - Transmit-receive switch receiver off characteristic with frequency. 
Figure 3.29 shows the receiver OFF characteristic when the switch is in transmit mode. As stated above, in this mode the receiver is isolated from the power amplifier to prevent damage during transmit. An input power of $-20 \mathrm{dBm}$ was inserted into the receiver and as can be expected, significant attenuation occurs across the spectrum at the frequencies of interest. For $10-100 \mathrm{MHz}$ at least $60 \mathrm{~dB}$ of attenuation occurs. $-80 \mathrm{dBm}$ was the noise floor of the analyser for the measurement. The attenuation is lowest at $500 \mathrm{MHz}$ where it reduces to approximately $38 \mathrm{~dB}$ caused by the PIN diode and stray board capacitances. Beyond $600-700 \mathrm{MHz}$ the roll off of the LNAs and coupling capacitors cause the increase in attenuation. The receiver noise characteristic is identical to that of the $500 \mathrm{MHz}$ receiver board in section 3.3.5.

The switching time of the circuit was $2.5 \mu \mathrm{s}$. There was coupling of the TTL SYNC signal to the transducer receive path during switching between states. This had no influence on the transducer signal path since any received signals were delayed by at least $12 \mu$ s due to the delay line of the transducers and the length of time for the ultrasound to travel the focal distance of the transducer in water. The main source of jitter in the system was due to the SYNC signal from the function generator since the entire system was synchronised on this signal. The jitter of this signal however was approximately 230 ps thus having very little effect.

\subsection{System Software}

The software controlling the system was a custom in-house designed graphical user interface (GUI) used to coordinate the generation, capture, and processing of the ultrasound signals used to characterise the sample materials.

\subsubsection{The GUI}

The system GUI was written in the programming language Delphi. This communicated with the function generator and oscilloscope using an Ethernet interface, allowing fast data transfer to and from these. A screen shot of the GUI plotting captured data from the scope is shown in Figure 3.30. This plot shows data captured from both channel $1(\mathrm{CH} 1)$ and channel $2(\mathrm{CH} 2)$ of the scope. The data from each channel was plotted in the same colour as is displayed on the scope. The yellow trace is the source waveform generated by the function generator, in this case a thirty percent windowed 20-80 MHz chirp. 


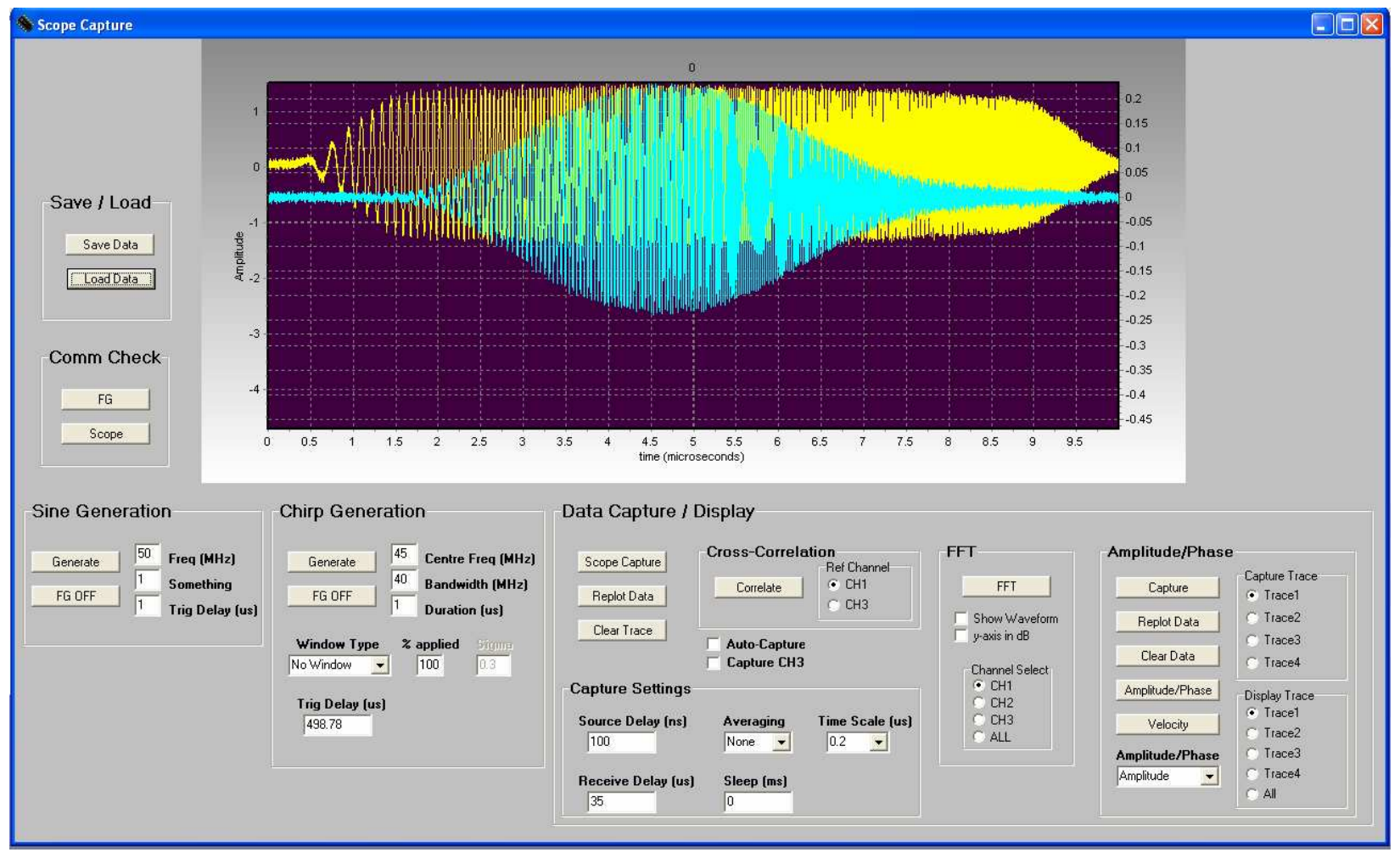

Figure 3.30 - System GUI displaying data captured from $\mathrm{CH} 1$ and $\mathrm{CH} 2$ of the oscilloscope.

The blue trace shows the pulse from the receiving transducer having passed through a water only path and then amplified by the receiver circuit. As observed, the GUI is separated into four main sections: Save/Recall, Chirp Generation, Sine Generation, and Data Capture/Display.

\subsubsection{Fast Fourier Transforms}

Fast Fourier Transform (FFT) magnitudes or phases can be viewed for any of the channels. Figure 3.31 shows the magnitudes of the FFTs of the waveforms shown in Figure 3.30. The FFT and IFFT (inverse FFT) algorithms were obtained from numerical recipes in Pascal [25]. The bell shape of the received signal is significantly different to that of the transmitted with a lower centre frequency. The bell shape is caused by the frequency response of the transducers. Example frequency responses of the $50 \mathrm{MHz}$ transducers provided by Olympus (section 3.2.3) exhibit this shaped response. The shifting of the centre frequency is caused by the absorption of water (see section 2.5.2) causing the higher frequency components to be greater attenuated. Also the centre frequencies of the transducers are not $50 \mathrm{MHz}$, but instead approximately 40-44 MHz. Waveforms can be captured from $\mathrm{CH} 2$ at different times and stored in four memory slots. 


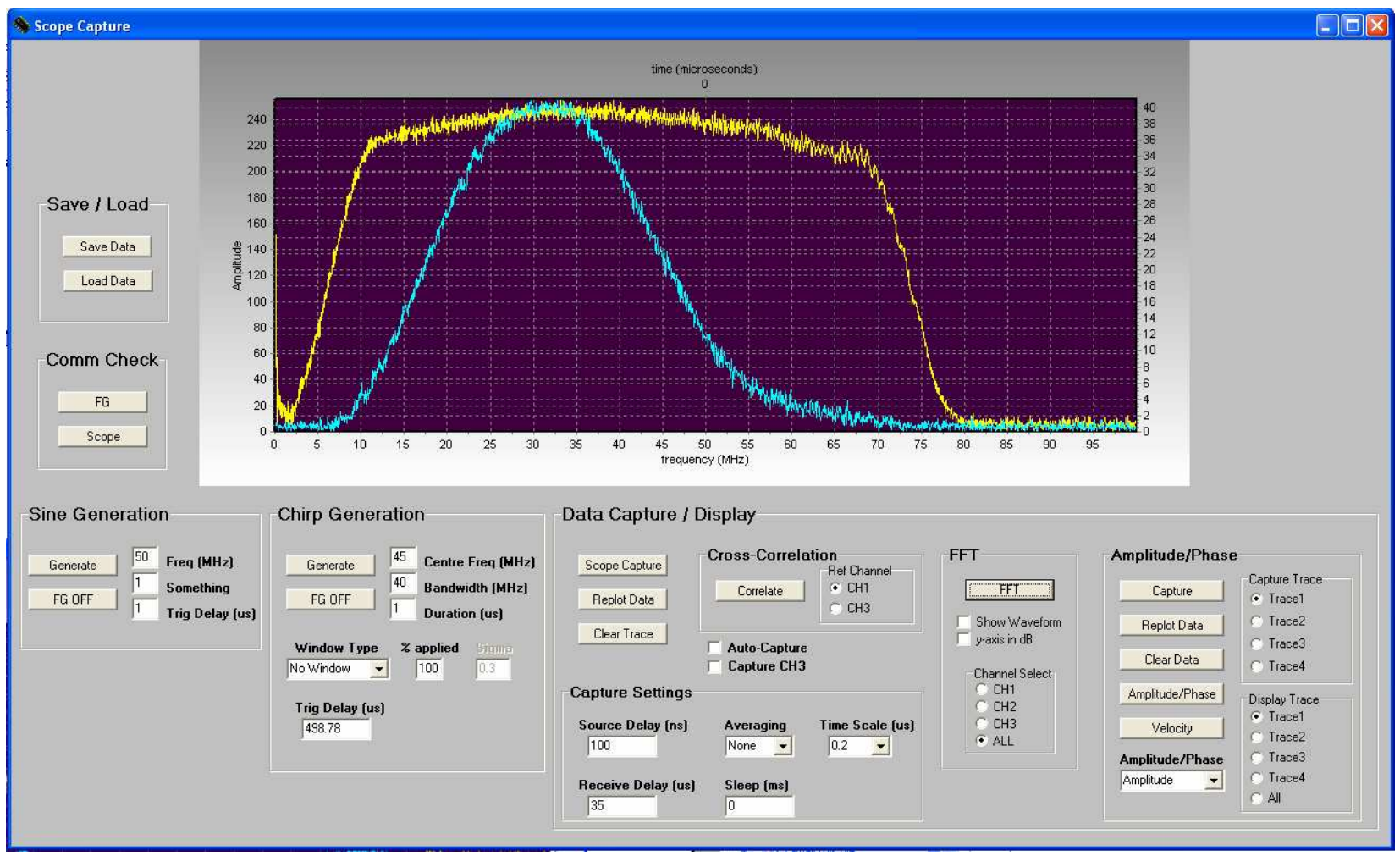

Figure 3.31 - Magnitudes of FFTs performed on $\mathrm{CH} 1$ and $\mathrm{CH} 2$ data.

This allows FFT magnitude and phase information of four waveforms to be compared and used to calculate phase velocity, attenuation coefficient, and acoustic impedance variations with frequency. Either the amplitude/phase/both of an individual wave can be displayed, or all four waveforms at once.

\subsubsection{Cross Correlation}

Cross correlation allowed time differences between the transmitted and receive pulses to be found to within tens of nanoseconds accuracy. Figure 3.32 shows the cross correlation result of the transmitted and received waveforms in Figure 3.30. The blue trace shows the received trace, and the green the cross correlation result. Cross correlation or matched filtering [88] is the optimal technique for detecting a known waveform in random white noise. The green cross correlation peak in Figure 3.32 is very large as the transmitted $50 \mathrm{MHz}$ wave is only passing through $26 \mathrm{~mm}$ of water before being received. It therefore suffers a small amount of attenuation due to absorption in the water, resulting in a large signal to noise ratio, corresponding to a large correlation peak. By identifying the time when the cross correlation peak is maximum by manually zooming in on the plot, and knowing the scope delay time as 


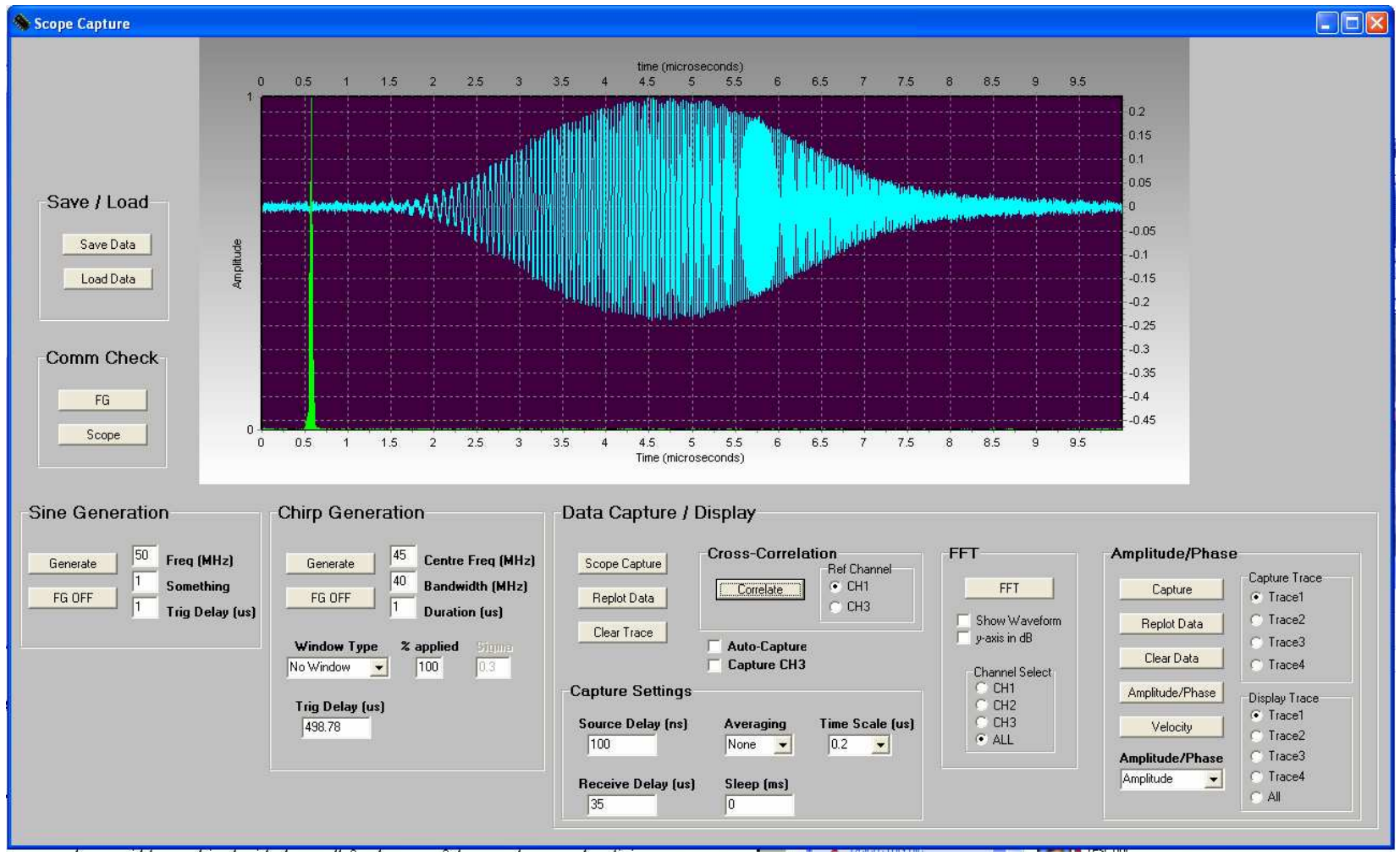

Figure 3.32 - Cross Correlation of received $\mathrm{CH} 1$ and $\mathrm{CH} 2$ data.

selected by the user on the GUI time differences between the transmitted and received signals can be found. The time resolution of the cross correlation was 1 ns. The cross correlation algorithm was implemented by reversing the transmitted signal in time, and then performing the convolution of the source and received waveforms in the frequency domain, as described in [89].

\subsection{3-axis Servo Motor Positioning System}

The narrow transducer beam widths combined with small focal zones made manual transducer alignment for a transmission measurement difficult and time consuming, especially for nonnormal angles of incidence. The Thorlabs LNR50VK3/M three-axis $50 \mathrm{~mm}$ TravelMax stage along with the three channel BDC103 DC motor drive was used with a resolution step size of $500 \mathrm{~nm}$. A NR360S rotary stage with BSC101 stepper motor drive was obtained that can be attached to the 3-axis stage. This allowed the angle of incidence of one of the transducers to be varied with precision. The two motor drives interface to the controlling PC via USB. The user interface used to operate the motors is the free APT user suite supplied by Thorlabs. 


\section{Chapter 4}

\section{Porous Anodic Alumina (PAA) Matching Layers - Fabrication and Structure}

To investigate the waveguiding influence of anisotropic microstructure on high frequency ultrasound, highly collinear porous anodic alumina (PAA) samples were fabricated. This allowed for experimental verification of the finite element modelling (FEM) performed for high frequency wave propagation in collinear microstructured materials. Once the wave propagation in an ideal PAA type structure is understood in the FEM and confirmed experimentally, understanding of the wave propagation in anisotropic materials with less regularity and collinearity can then be considered e.g. fibrous tissues. In this chapter the anodisation process for the fabrication of the PAA samples is described. For further background on the formation of porous anodic alumina on aluminium see [90]-[93].

Unexpected microstructure can be encountered during the non-destructive testing of seemingly homogeneous materials. Solid aluminium is an example that readily oxidises at its interfaces to produce aluminium oxide or alumina. This alumina layer is very thin (of the order of nanometers) but consists of a highly aligned regular porous structure. Taking advantage of this property, porous collinear alumina membranes with ten to hundreds of nanometer-sized pores and pore wall dimensions can be fabricated by anodising high purity aluminium using a controlled and repeatable anodisation process. 
Such a highly regular structure constrains the propagation of high frequency ultrasound, resulting in consistent guided wave propagation. By modifying the fabrication process parameters, the structural properties of the PAA samples (e.g. pore spacing, sample architecture) can be modified. These modifications can lead to variations in the acoustic properties, allowing the tailoring of these samples for specific applications.

\subsection{PAA Sample Preparation}

As described in section 2.4, nanoporous anodic alumina samples have been a topic of interest for many years due to their unique and ideal nanostructure. Typical applications include templates for growing nanostructured materials, and for use as adhesion layers. The anodisation of high purity aluminium to produce nanoporous alumina structures is well understood and was the technique used in this work.

To make these PAA samples, ultra high purity aluminium (99.99\%) was used as the substrate material. This was cleaned chemically using ethyl acetate, ultrasonic cleaned for 10 minutes, washed with distilled water, and then left to air dry. Anodisation replicates a mirror image of the substrate surface, so the aluminium was pressed between two steel plates before use to ensure the anodising process took place on a flat aluminium substrate. Figure 4.1 shows the experimental setup used to anodise the PAA samples. The prepared aluminium sample was placed on a copper plate and then clamped between a cooled steel plate and a clamp-on cell. An o-ring separated the clamp on cell from the aluminium to seal off any leaks (the size of the oring determining the size of the resultant sample). An electrolyte was then poured into the clamp-on cell and stirred using a magnetic stirrer. Either sulphuric, oxalic, or phosphoric acid with $0.3 \mathrm{molL}^{-1}$ concentration was used as the electrolyte. It was found that the electrolyte determines the pore dimensions of the samples. A DC current was passed through the electrolyte by setting the anodising voltage required for the particular electrolyte used. The positive terminal was connected to the copper plate and the negative terminal connected to a platinum electrode that was immersed in the electrolyte as shown Figure 4.1. With the voltage applied, the system was left for the period of time required for the anodising process to take place, depending on the desired sample thickness. Anodisation time with sample thickness is an exponential relationship. Samples of $100 \mu \mathrm{m}$ thickness took approximately 20 hours, whilst samples of $1 \mathrm{~mm}$ thickness would take at least a couple of weeks to prepare. Typical sample 
thicknesses used were between 60-500 $\mu \mathrm{m}$. The anodisation current was monitored during the process as an indication of when the desired thickness was obtained. The current decreased exponentially with anodisation depth with the values of anodising current dependant upon the electrolyte used e.g. for sulphuric acid at $2.5 \mathrm{~mA}$ the thickness of the PAA samples is $150 \mu \mathrm{m}$, for oxalic acid the current is $5 \mathrm{~mA}$ for that same thickness. Once the desired thickness was reached the clamp on cell was then removed and the samples placed into iodine-methanol solution to remove any excess metallic aluminium. All samples produced were robust and could be handled comfortably using standard tweezers.

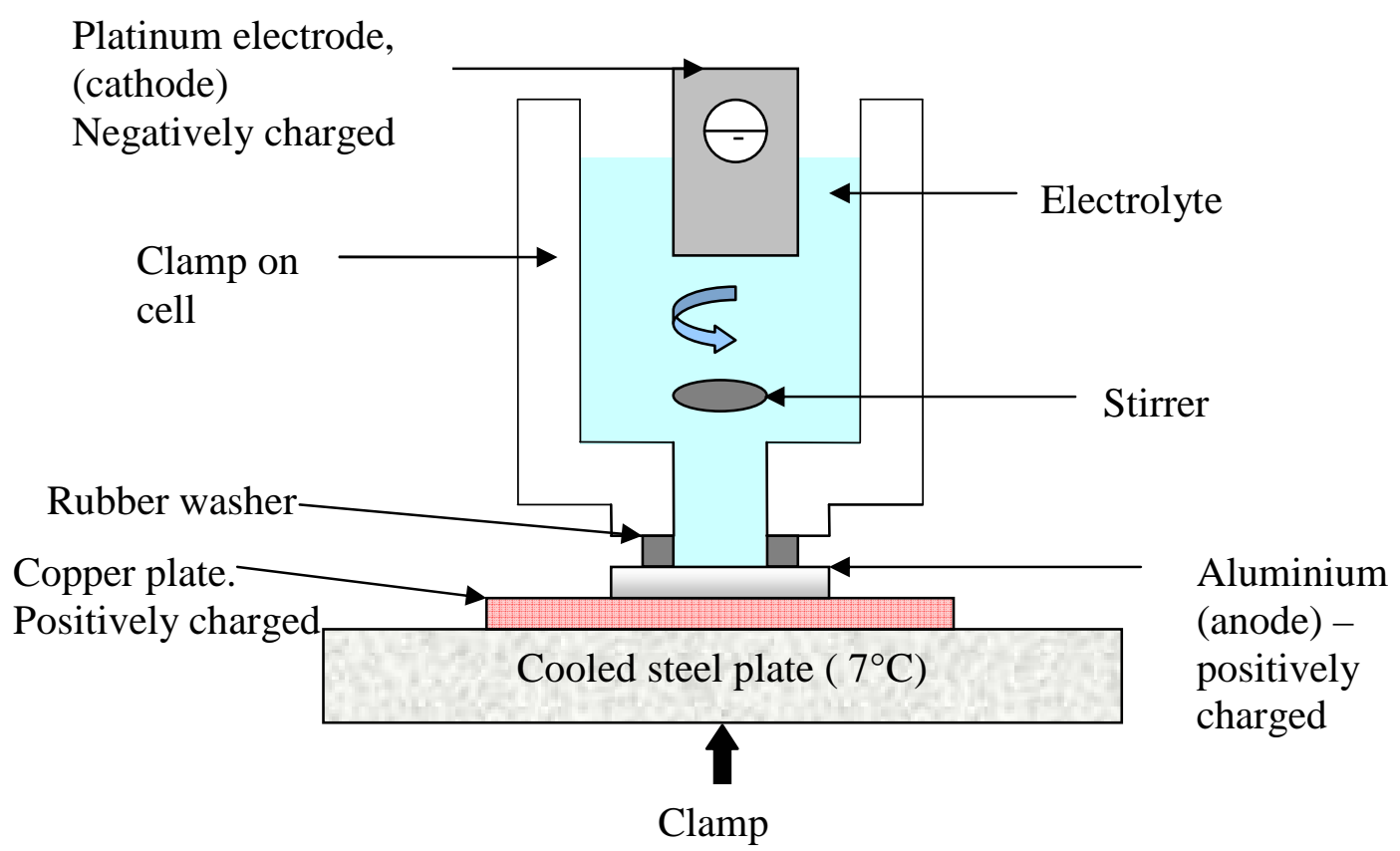

Figure 4.1 - PAA sample preparation setup.

The method used here followed the main steps of most standard PAA anodisation procedures, with the major exception being the samples fabricated for this work did not undergo heat treatment, the final step usually performed when producing PAA structures. Heat treatment as described in the literature from section 2.4 causes the transformation from amorphous to crystalline alumina. Crystalline alumina phases possess larger elastic constants than the amorphous phase. In this work the amorphous phase was desired and was confirmed for our samples using X-Ray diffraction and NMR spectroscopy [63]. No crystalline structure was observed until heat treatment temperatures of $800{ }^{\circ} \mathrm{C}$ or above. 
Figure 4.2 shows the variation in interpore spacing as a function of the anodising voltage and the electrolyte used. As can be observed there is a finite voltage range each electrolyte can support for the anodising process to occur. The sulphuric acid electrolyte produces samples with the smallest interpore spacing using the smallest anodising voltages. Oxalic acid is very close to this with a slight increase in anodising voltage and similar spread in interpore spacing. A very large increase in anodising voltage is then required when using phosphoric acid producing significantly larger inter pore spacing. An incorrect voltage applied to an electrolyte causes poorly formed anodic layers on the aluminium substrates. The general rule found was the pore spacing is approximately proportional to 2.5 times the applied voltage. However there is some statistical spread, giving rise to the range of different pore spacings possible for a given anodising voltage as observed in Figure 4.2. These results were obtained from in-house measurements of many samples prepared using the three different electrolytes, more detail on this relationship can be found in [94] and [95]. The pore size appears closely related to the pore spacing, however it is also influenced by the concentration of the electrolyte and the temperature at which the process takes places. Both of these were kept constant in this work.

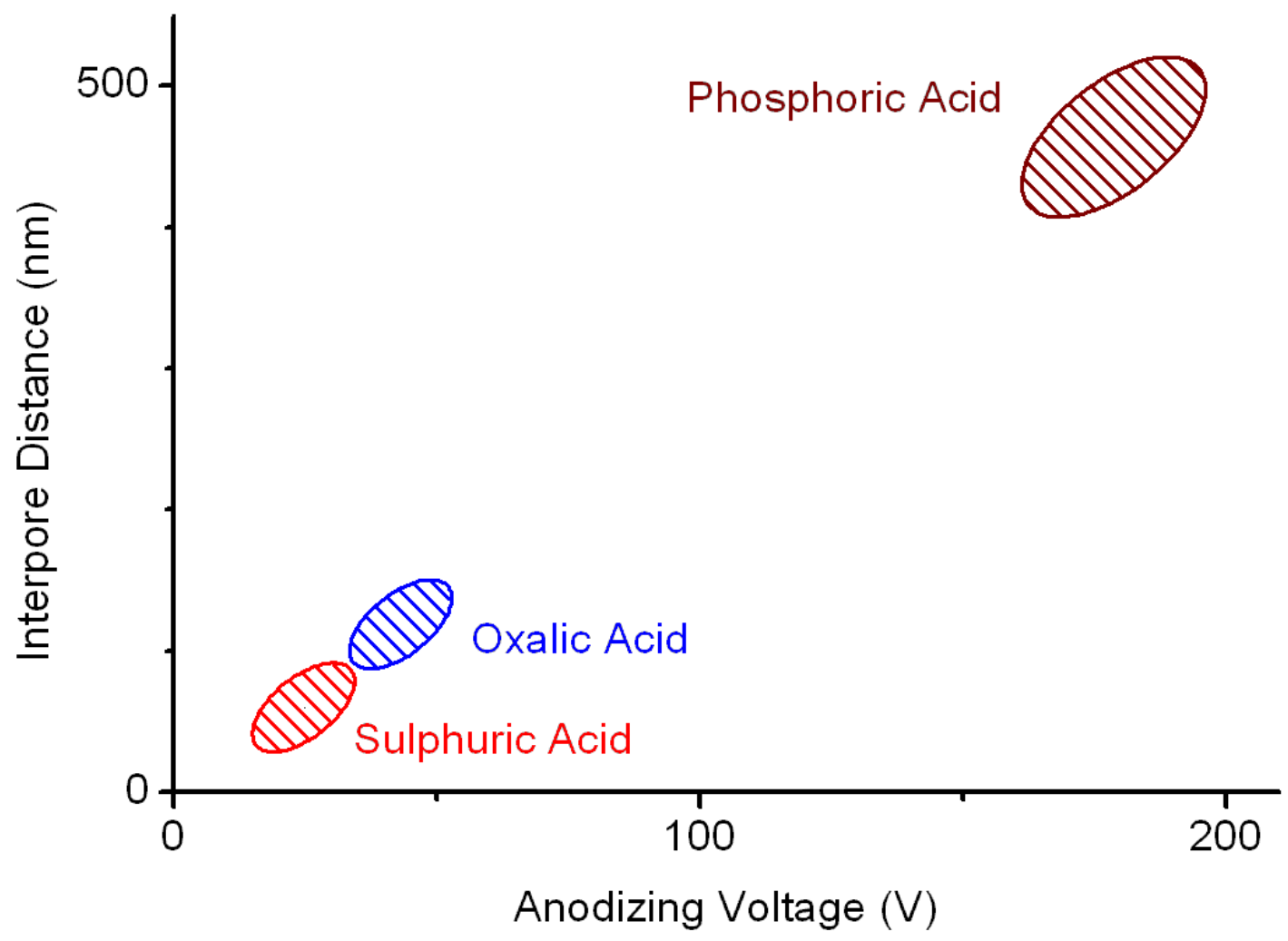

Figure 4.2 - PAA interpore distance variations with anodising voltage for the sulphuric, oxalic, and phosphoric electrolytes. 


\subsection{PAA Sample Structure}

Example horizontal and vertical cross-sections of all three PAA sample types and a less regular commercial PAA sample are now presented. It should be noted that all four sample types were hydrophilic, absorbing water into the pores upon immersion. Figure 4.3 shows the horizontal cross section of a sample prepared using sulphuric acid as an electrolyte where the highly regular structure is observed. Samples prepared using a sulphuric acid electrolyte are called type 1 samples. All the pores are circular and possess the same diameter of approximately 30 $\mathrm{nm}$. The pores are regularly spaced from pore centre to pore centre approximately $60 \mathrm{~nm}$ apart. Each pore is arranged in a hexagonally close packed (HCP) arrangement relative to the surrounding pores.

The porosities of these type 1 samples were measured in the range $23-28 \%$ by performing greyscale threshold detection image processing in Matlab. This was performed on several SEM images for the same sample type with the results averaged. The SEM images were read into Matlab with a user defined brightness threshold set to distinguish whether a pixel of the image was black or white (pore or wall). These tests were carried out on every pixel converting each to either black or white based on the test. Using the new recreated image the porosity was determined by calculating the total number of black pixels divided by the total number of (black plus white) pixels. Figure 4.4 shows the longitudinal cross section where a sample has been fractured by physically snapping it in half. This accounts for the broken pores that are visible. What can be observed is how highly collinear the samples are where pores run directly from top to bottom with no irregularities in between. The inset picture shows a zoomed in version of the pores where the collinearity of the sample is better observed. The longitudinal cross sections for the other two types of fabricated samples are visually the same with the same level of collinearity, the only difference being the dimensions of the pores are different so they are not shown. 


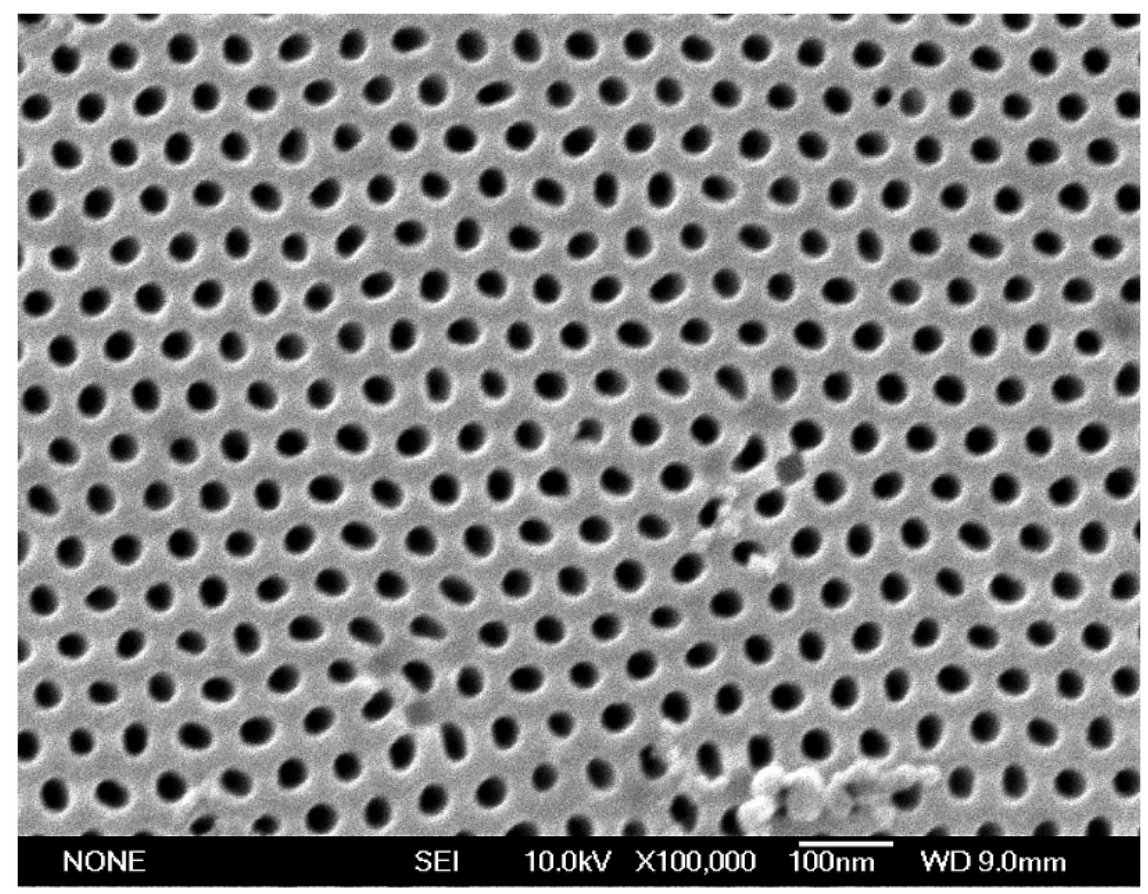

Figure 4.3 - Horizontal cross section of a sulphuric acid prepared porous alumina sample (type 1 sample) showing $30 \mathrm{~nm}$ sized pores with $60 \mathrm{~nm}$ spacing.

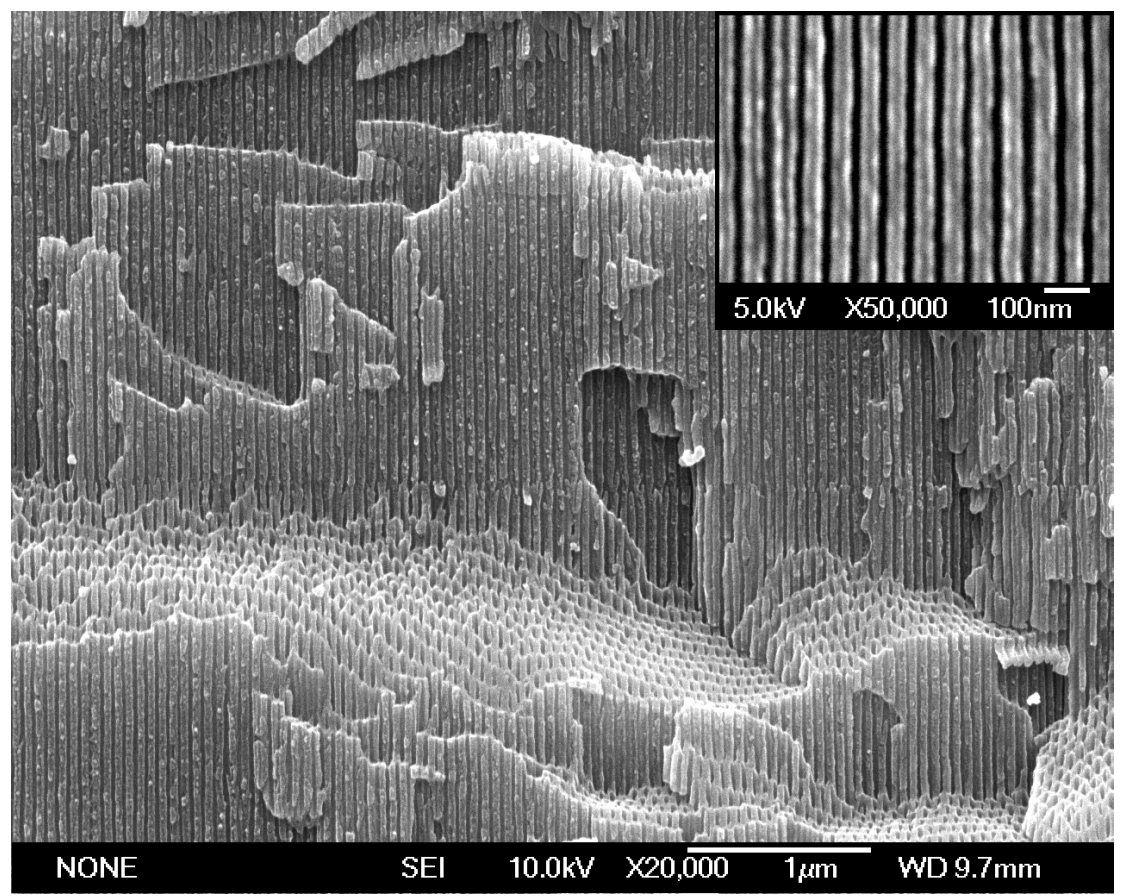

Figure 4.4 - Longitudinal cross section of a sulphuric acid prepared porous alumina sample (type 1 sample) showing the highly collinear structure of these samples. 
The samples prepared using oxalic acid as an electrolyte are called type 2 samples. These possessed slightly larger pore dimensions and spacing than the type 1 samples but the main difference is they exhibited a different architecture. Figure 4.5 shows a horizontal cross section of a type 2 sample. The pores here are not circular as in the type 1 sample, but instead appear more hexagonal in structure. The regularity is still similar for these samples where the solid separating the pores is of uniform thickness. This solid looks like many plates of uniform thickness joined together, quite different when compared with Figure 4.3. Since this is the case a different model was used to simulate these samples. The pore diameters are larger at approximately $40 \mathrm{~nm}$, and these are spaced approximately $80 \mathrm{~nm}$ apart. These samples are also much more porous than the type 1 samples with measured porosities in the range $40-44 \%$.

Finally the samples prepared using phosphoric acid as an electrolyte possessed the largest sample pore/spacing dimensions out of the three samples. These are called type 3 samples. Figure 4.6 shows a horizontal cross section of one of these samples. The pores in this cross section are of no particular shape or regularity. On average, at the widest points these pores are approximately $400 \mathrm{~nm}$ wide and spaced approximately $500 \mathrm{~nm}$ apart centre to centre. The solid separating the pores is of consistent thickness throughout the sample showing a likeness in architecture to the type 2 samples. These walls are approximately $200 \mathrm{~nm}$ thick, with the solid separating the pores like many uniform thickness plates. These samples are the most porous with measured porosities in the range $46-50 \%$.

Porous alumina membranes can be purchased commercially. Figure 4.7 and Figure 4.8 show horizontal and longitudinal cross sections of a commercial sample obtained from Whatman [106]. As can be seen, these samples display significantly less regularity in pore size, pore spacing, and collinearity, in contrast to those fabricated in-house. They possess an average pore size of approximately $200 \mathrm{~nm}$, but the solid separating the pores is not of consistent thickness throughout the sample. The longitudinal cross section reveals the samples are not highly collinear where pores do no extend from the top of the sample to the bottom. This can be observed in Figure 4.7 as well where the tops of some pores do not penetrate the surface of the sample. These samples required much more care when handling to avoid fracturing. 


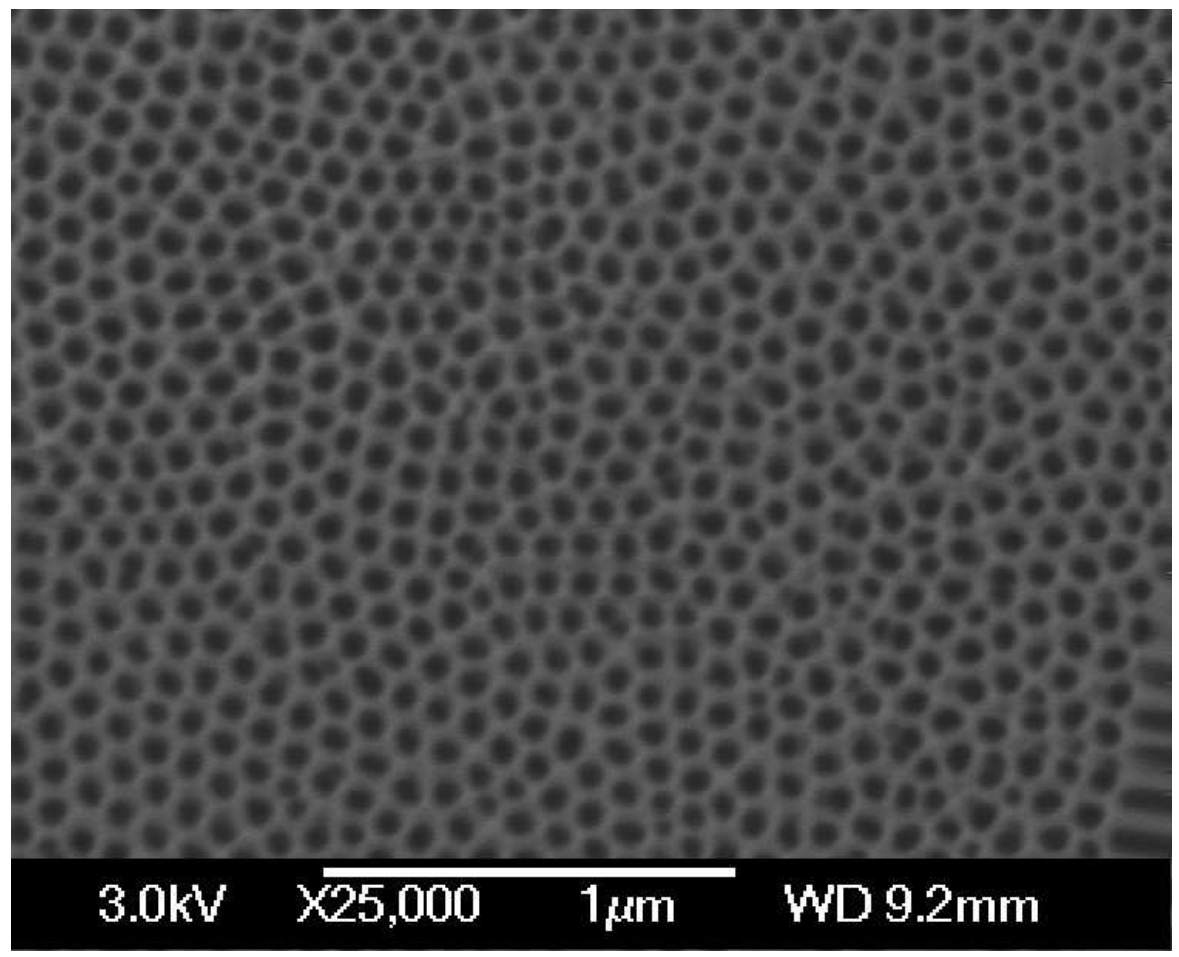

Figure 4.5 - Horizontal cross section of an oxalic acid prepared porous alumina sample (type 2 sample) showing $40 \mathrm{~nm}$ sized pores with $80 \mathrm{~nm}$ spacing.

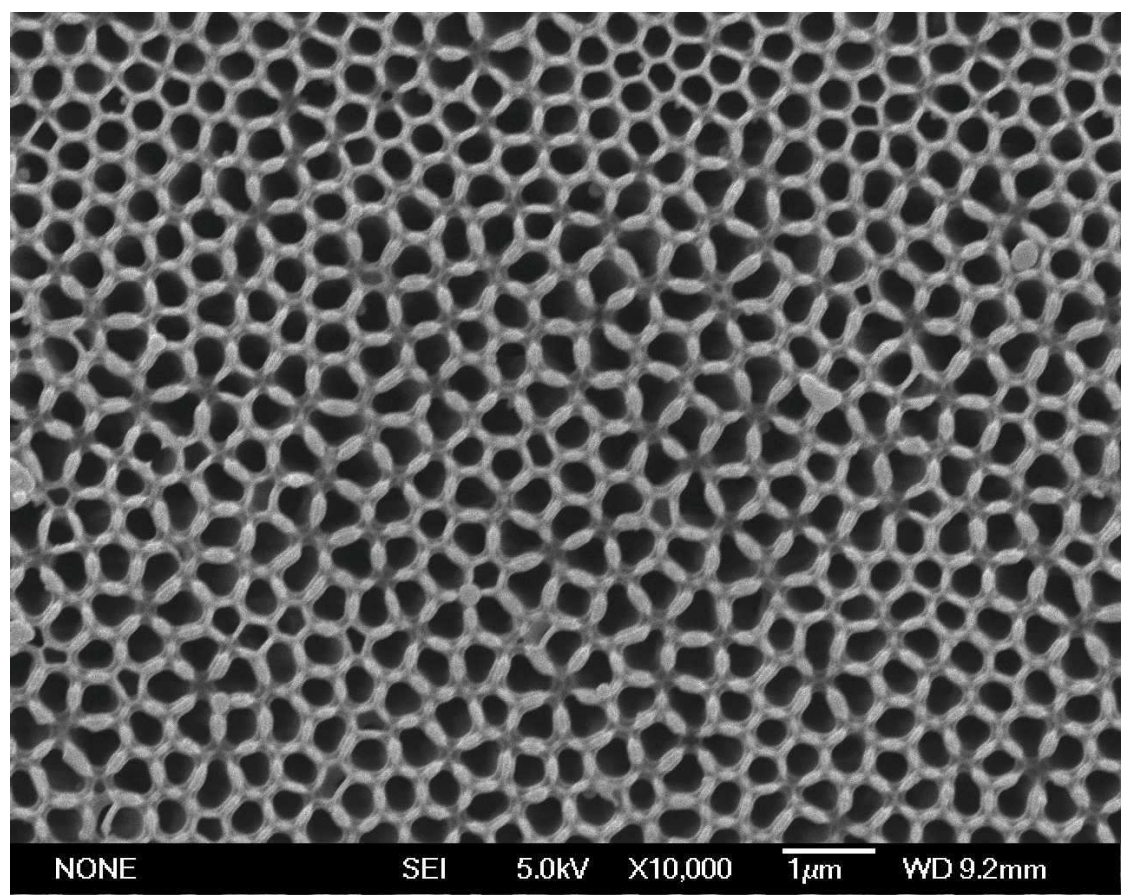

Figure 4.6 - Horizontal cross section of a phosphoric acid prepared porous alumina sample (type 3 sample) showing on average $400 \mathrm{~nm}$-sized pores with $500 \mathrm{~nm}$ spacing. 


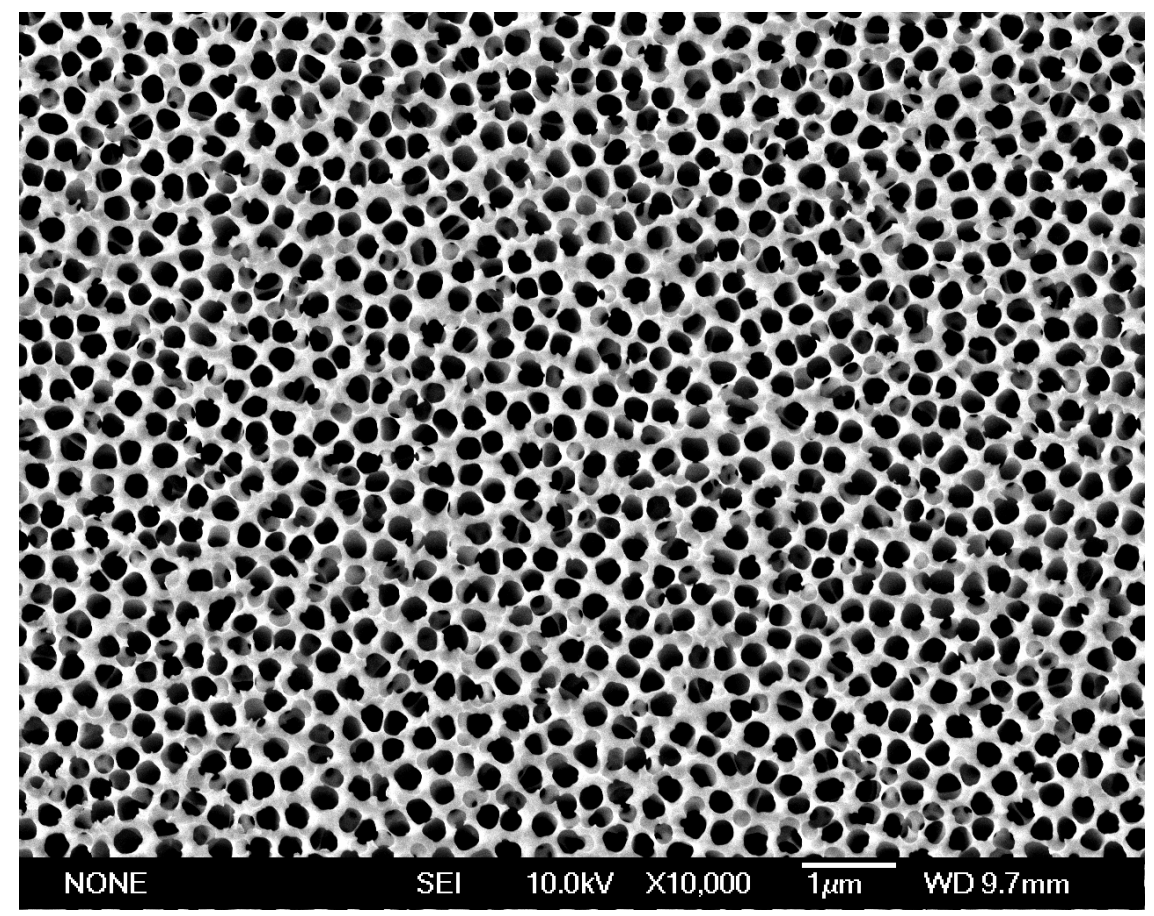

Figure 4.7 - Horizontal cross section of commercial porous alumina sample.

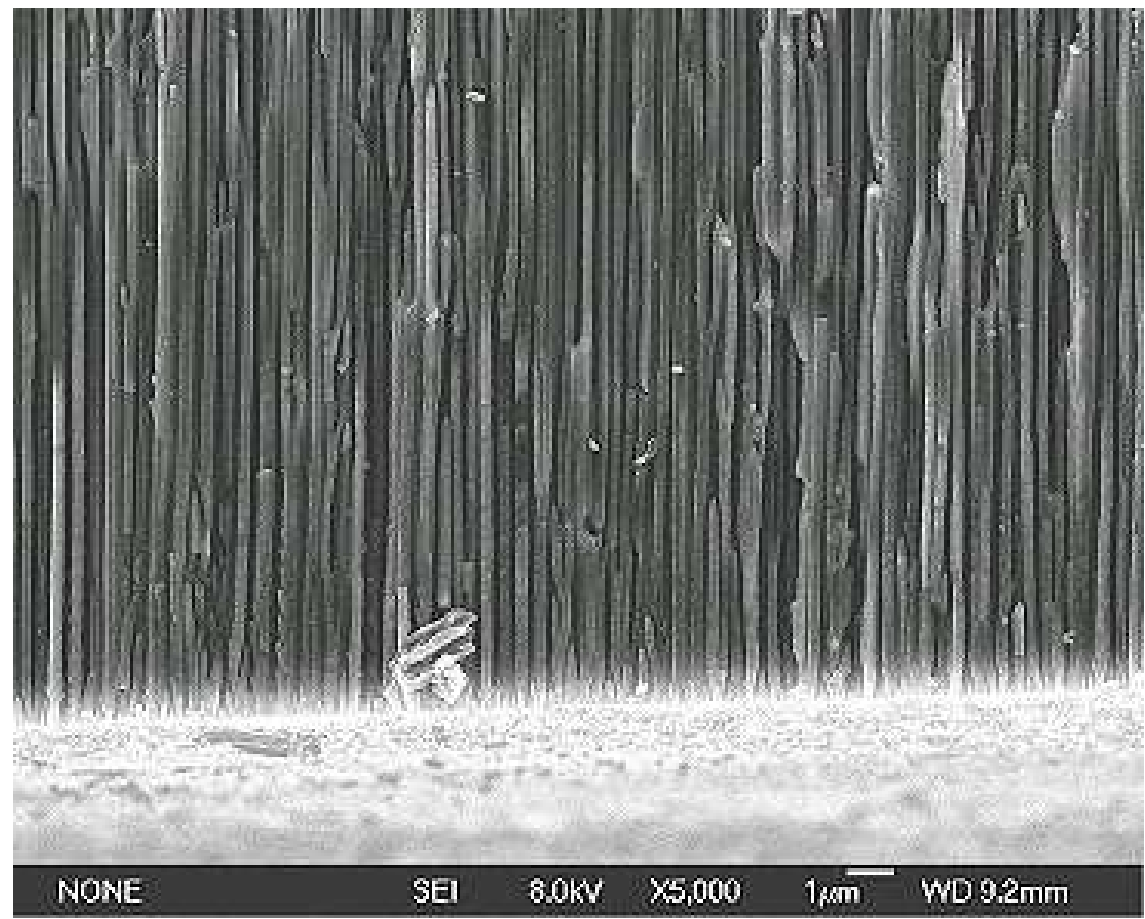

Figure 4.8 - Longitudinal cross section of commercial porous alumina sample. 


\section{Chapter 5}

\section{Finite Element Modelling of Collinear Porous Solids}

With the continual increase in computing technology leading to more powerful desktop and laptop PCs, the need for large parallel computer clusters for computationally expensive simulations is becoming less of a requirement. Also, with the development of dedicated and subject specific finite element modelling packages (FEM), desktop simulations with reduced FEM computation times are a reality. FEM simulations divide a physical problem into a grid of discrete elements (typically rectangular or triangular), each with user described material properties. Each element responds to a user-defined stimulus e.g. stress in the case of a mechanical simulation, to produce physical outputs from each element e.g. strain, according to the set properties and structure of the medium. Material properties in the case of mechanical systems are defined by their elastic constants e.g. Young's and shear modulus, or by their P and $\mathrm{S}$ wave speeds, and density. By increasing the number of elements the accuracy of the simulation is improved. For example, when generating a cylindrical structure using a rectangular grid, the more elements used the more realistic the cylinder. This results in an increase in computation time, especially for 3D simulations.

PZFlex [107] written by Weidlinger Associates, is an FEM package dedicated to piezoelectric and ultrasonic wave propagation applications. With the wide range of commands and tools 
dedicated to ultrasonic applications, a deeper fundamental understanding of a problem can be obtained in a time efficient manner before experimental work commences. In this work FEM was performed using PZFlex to investigate the wave guiding properties of highly regular structured solids on high frequency ultrasound waves. Sample dimensions, architectures, and wave frequencies were varied, and the impact of these changes observed. This same detailed understanding was not possible experimentally, where the variation in structural properties was limited to the three PAA samples fabricated. Modelling of ultrasonic wave propagation was performed in three phases

I. Preliminary simulations were performed for homogeneous media to confirm the expected bulk wave propagation in common liquids and solids.

II. 2D models were then performed on anisotropic media to provide an understanding of ideal guided wave propagation in highly structured solids.

III. 3D modelling was then performed to investigate wave propagation in more realistic representations of the fabricated PAA samples.

\subsection{Simulated Material Properties}

As stated in section 4.1, the PAA samples fabricated for this work were confirmed to be in the amorphous alumina phase. The exact bulk acoustic properties of amorphous alumina are not well published, however from [60], [62], and [108]-[110] it can be established the acoustic moduli of amorphous alumina are approximately 3.0-3.5 times less than those of crystalline alumina. The density of amorphous alumina was determined from [58], [62], and [109]. These properties result in longitudinal velocity, shear velocity, and density ranges slightly larger but similar to aluminium. Since uncertainty exists in the exact values of the amorphous alumina properties, the alumina was modelled as solid aluminium with acoustic properties obtained from [33]. This allowed a logical progression from confirming previous work from the literature on free boundary and fluid loaded aluminium plates towards 3D representations of the PAA structures using the same material properties. Even though using the acoustic properties of aluminium for amorphous alumina meant measurements (such as velocity) calculated from the simulations were not exactly equal to those in amorphous alumina, they still provided a good indication of the variations in values expected, and the waveguiding phenomena observed were still valid. 
Table 5.1 - Bulk acoustic properties of crystalline alumina, amorphous alumina, aluminium, and water.

\begin{tabular}{|c|c|c|c|c|}
\hline Material & $\begin{array}{l}\text { Density } \\
\left(\mathrm{kgm}^{-3}\right)\end{array}$ & $\begin{array}{l}\text { Longitudinal } \\
\text { Velocity }\left(\mathrm{ms}^{-1}\right)\end{array}$ & $\begin{array}{l}\text { Shear Velocity } \\
\left(\mathrm{ms}^{-1}\right)\end{array}$ & $\begin{array}{l}\text { Acoustic } \\
\text { Impedance } \\
\text { (MRayl) }\end{array}$ \\
\hline$\alpha$-Crystalline Alumina & 3860 & 10052 & 6350 & 40.6 \\
\hline Amorphous Alumina & $2700-3400$ & $6520-7150$ & $3330-3920$ & $17.9-24.3$ \\
\hline Aluminium & 2700 & 6300 & 3100 & 17.0 \\
\hline Water & 1000 & $\begin{array}{l}1480 \\
\text { (at room temp.) }\end{array}$ & 0 & 1.48 \\
\hline
\end{tabular}

Therefore only aluminium and water were the materials used in the simulations with the density, velocity, and acoustic impedance values of these shown in Table 5.1. Also shown are the ranges of velocities calculated for bulk amorphous and crystalline alumina. As can be observed the mechanical properties of crystalline alumina are significantly larger than amorphous alumina due to the improved order of the crystalline structure.

\subsection{Single Free Boundary Aluminium Plate}

The first simulation performed was a simple $2 \mathrm{D}$ model used to analyse the group velocity dependence upon incident wave frequency and plate thickness for end on excitation of a single free boundary aluminium plate. The model used is shown in Figure 5.1.

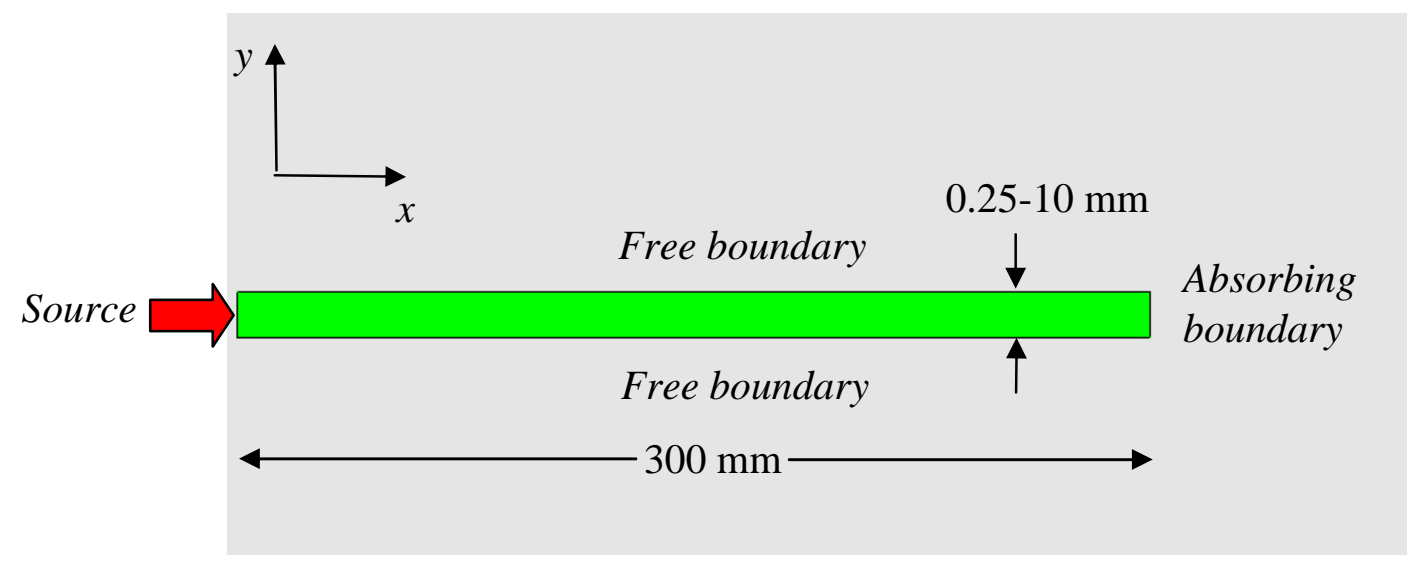

Figure 5.1 - Finite element model of a single free boundary aluminium plate with boundary conditions shown (dimensions not to scale). 
This is the simplest model confirming the original theoretical work performed by Lamb and others [19], [21], [22], [24]. Model boundary conditions are shown for all plate boundaries. The boundary at the right end is fully absorbing preventing any reflections. The source is applied to the entire left boundary, resulting in plane wave excitation. The source pulse used for all simulations was a Blackman pulse. The Blackman pulse is the functional form of the second derivative of the Blackman-Harris window [111]. It is a temporally compact pulse whose frequency content is a Gaussian distribution centred at the selected wave frequency. Figure 5.2 shows the Blackman pulse with a centre frequency of $100 \mathrm{kHz}$ used for this simulation in both the time and frequency domain. The frequency spectrum shows a bandwidth just over $200 \mathrm{kHz}$.
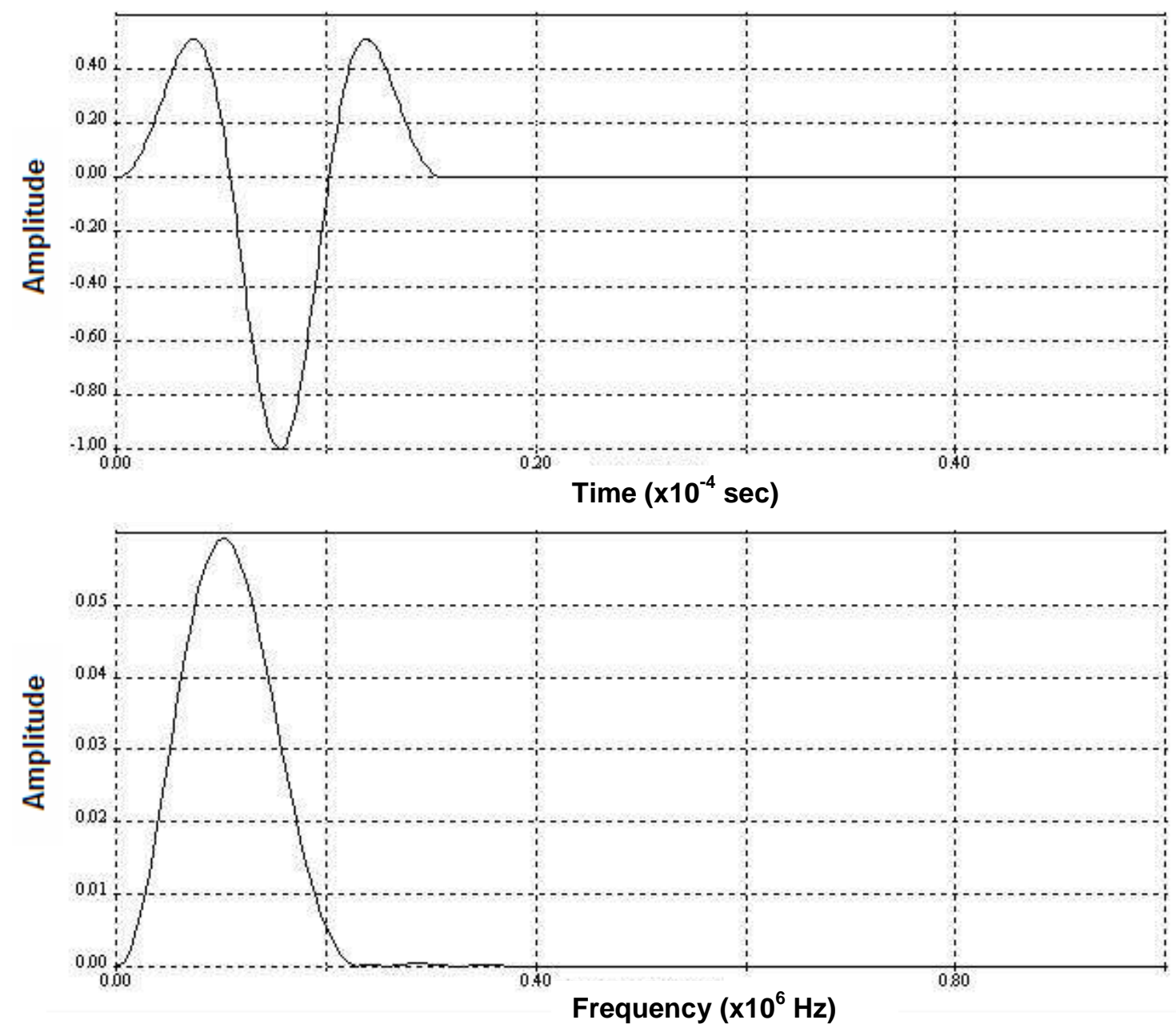

Figure 5.2 - Blackman pulse in time and frequency domains.

Referring to the symmetric mode group velocities shown in Figure 2.5 of section 2.1.3, the Lamb wave velocity in a free plate varies significantly with the frequency-thickness $(f d)$ product. The frequency thickness product for this simulation was varied from 
0.025 to $1 \mathrm{MHz} . \mathrm{mm}$, and the group velocity was measured over this range. This represents the range over which the $f d$ product varies with the transducer frequencies and PAA wall thickness dimensions used in the experimental work. This was achieved in the model by fixing the source frequency at $100 \mathrm{kHz}$, then varying the plate thickness from 0.25 to $10 \mathrm{~mm}$. The FEM element size used was $62.5 \mu \mathrm{m}$ to ensure enough spatial resolution for the smallest plate thickness of $0.5 \mathrm{~mm}$. The plate length was $300 \mathrm{~mm}$ where the longer the plate the more accurate the velocity measurement at the expense of greater computation time. $300 \mathrm{~mm}$ equates to 4800 elements long, which is more than sufficient to obtain a fair measurement. The velocity was determined by measuring the time difference between pulse peaks at two different ends of the plate. Using this time difference and the known plate length, the plate group velocity $v_{\text {plate }}$ is given by

$$
v_{\text {plate }}=\frac{L}{t_{\text {end }}-t_{\text {beg }}}
$$

where $t_{\text {beg }}$ is the time at which the power flux peak occurs at the beginning of the plate, $t_{\text {end }}$ the time at which the power flux peak occurs at the end of the plate, and $L$ is the length of the plate.

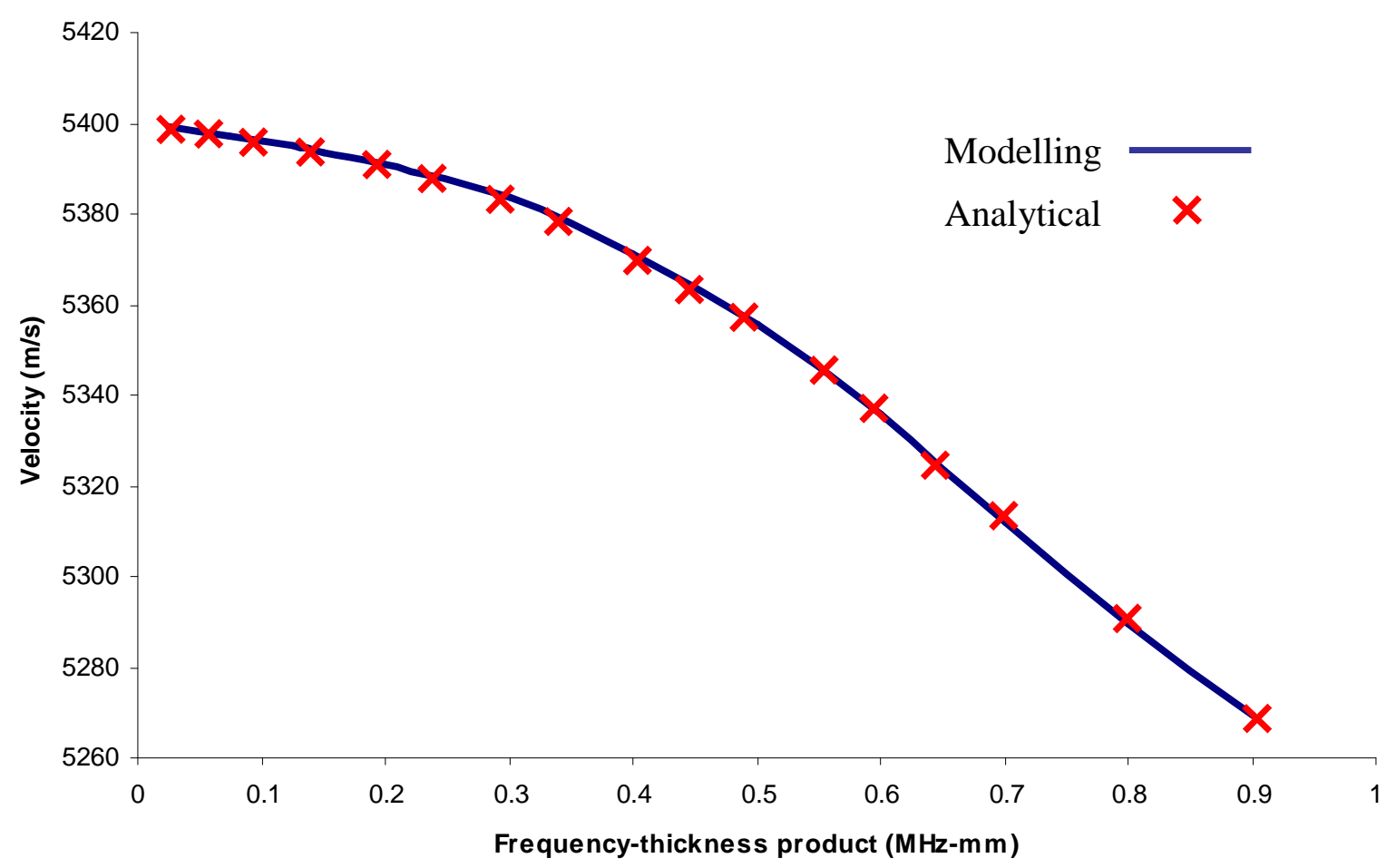

Figure 5.3 - Group velocity in a free boundary aluminium plate as a function of the frequencythickness product for end on excitation. 
Figure 5.3 shows the measured variations in group velocity with $f d$ product obtained from the simulations plotted with select points obtained using the analytical equations from section 2.1.3. The analytical and modelling overlay in excellent agreement with the expected result that the $f d$ product has a significant influence on the plate wave group velocity. For near zero $f d$ products, the velocity of the wave is given by equation (2.5). Using this equation results in a velocity of $5400 \mathrm{~ms}^{-1}$, agreeing with that observed in Figure 5.3. The fact the source is excited at normal incidence eliminates the possibility of the fundamental anti-symmetric mode, since the wave can only consist of longitudinal displacements. Since the $f d$ product is so low, the existence of higher order modes is not possible. However, due to the broadband nature of the Blackman pulse, for larger plate thicknesses, dispersion of the wave was evident. This is observed in Figure 5.4 showing images of the pulse pressure in the plate at (a) the beginning of all plates, and upon reaching the end of the plate for thicknesses of (b) $1 \mathrm{~mm}$, (c) $5 \mathrm{~mm}$, and (d) $9 \mathrm{~mm}$.

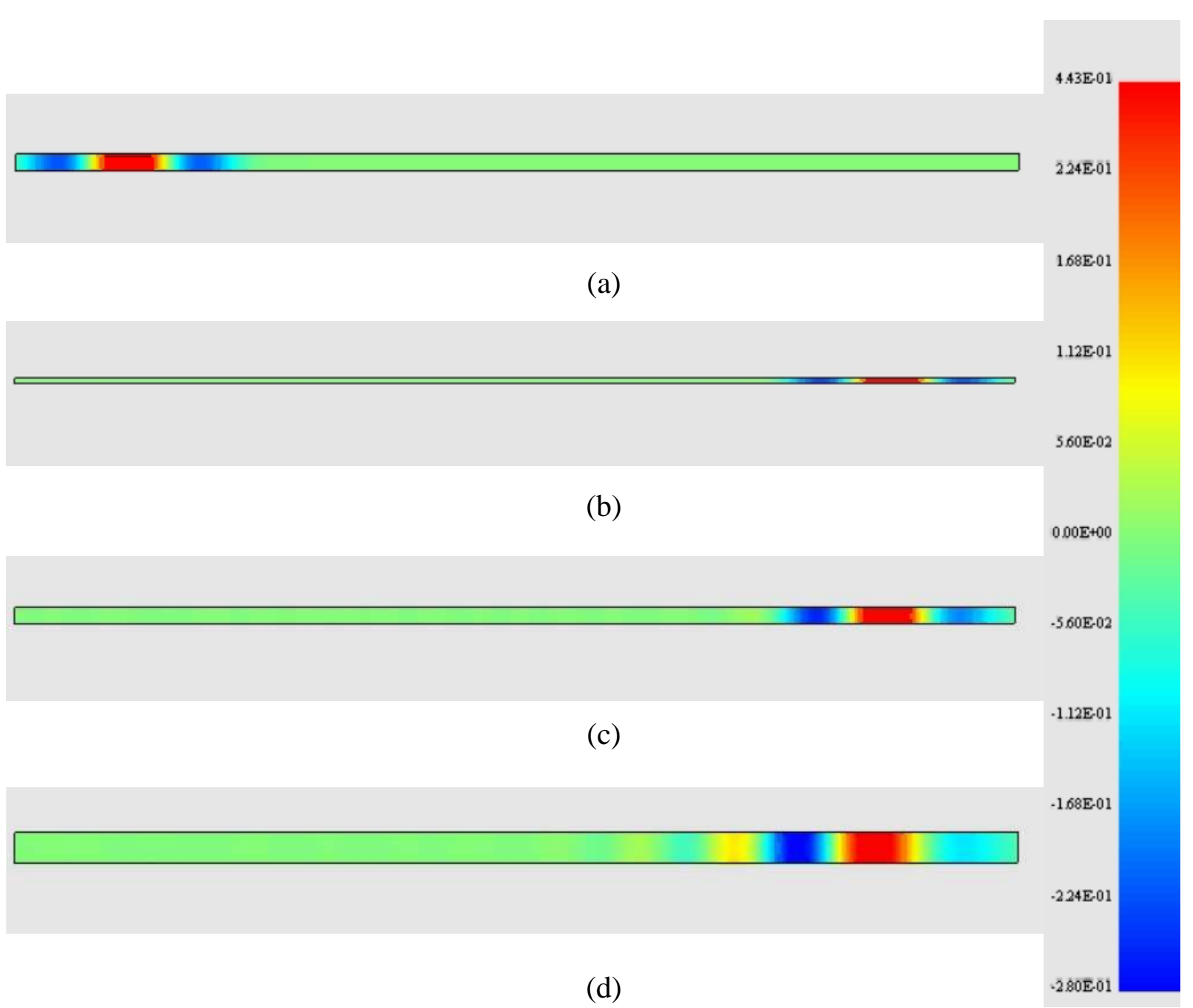

Figure 5.4 - Pulse pressure in the free boundary aluminium plate at (a) the beginning of all plates, and at the end of (b) $1 \mathrm{~mm}$, (c) $5 \mathrm{~mm}$, and (d) $9 \mathrm{~mm}$ thick plates. 
In these plots the pulse pressures are represented by a colour as indicated in the colour scale on the right. The same scale was used for each plate where navy blue is the maximum compression applied by the pulse and red the maximum expansion. Figure 5.4 (a) shows the source pulse at the beginning of an arbitrary thickness plate. The thickness is irrelevant here since the source pulse is the same at the beginning of all plates. Here the two navy blue components of the pulse correspond to the two positive peaks of the Blackman pulse in Figure 5.2 and red the large negative peak. Observing the pressure at the ends of the three different plates (b)-(d), the energy in the waveform becomes significantly more dispersed as the width of the plate is increased, where for the $9 \mathrm{~mm}$ plate significant energy can be seen to trail behind the original main pulse.
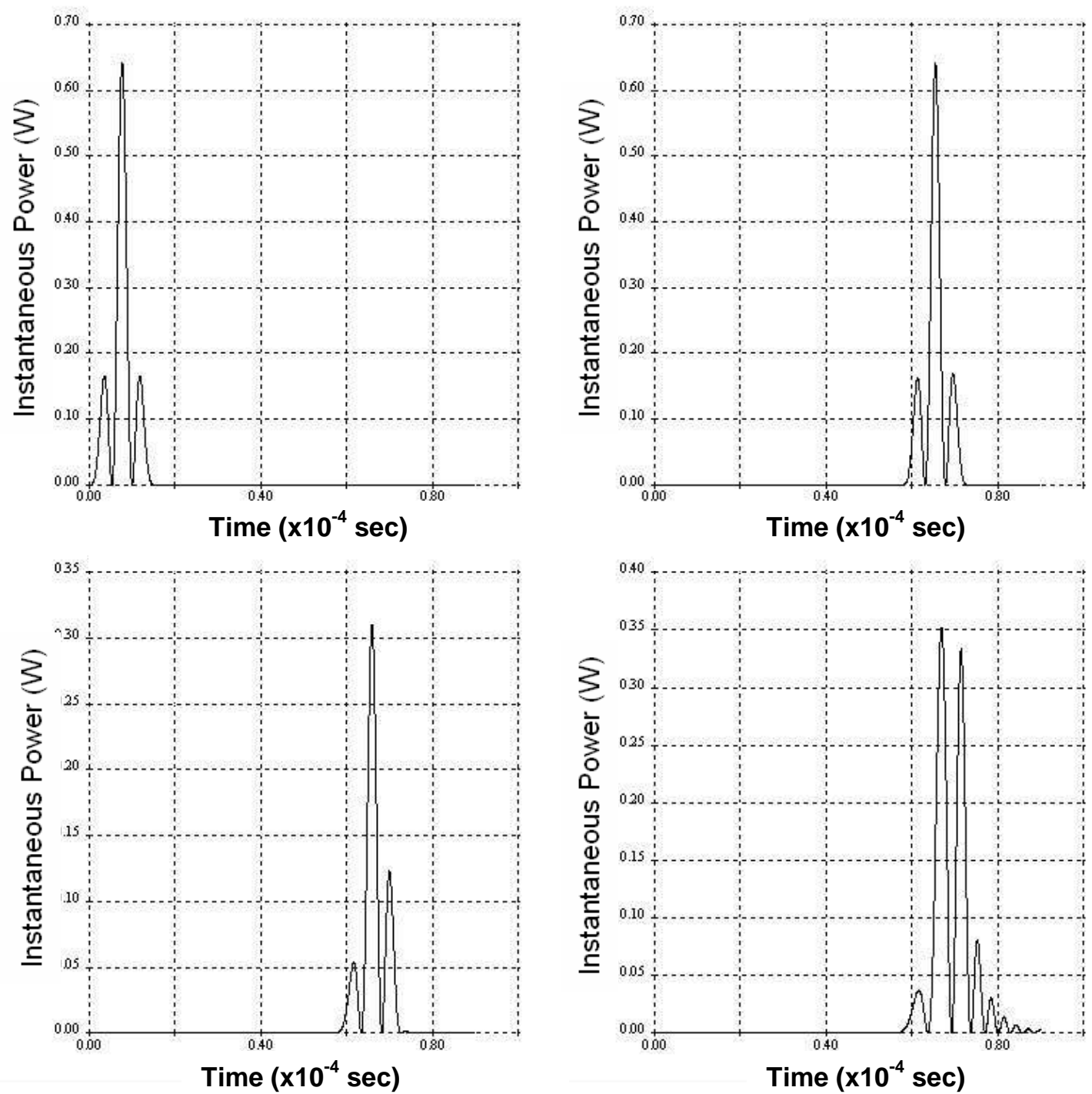

Figure 5.5 - Power through a vertical line of plate nodes at the beginning (top left) of all plates, and upon reaching the end of a $1 \mathrm{~mm}$ (top right), $5 \mathrm{~mm}$ (bottom left), and $9 \mathrm{~mm}$ (bottom right) thick plates. 
Figure 5.5 shows an alternative way to observe this dispersion by plotting the power through a vertical line of nodes across the thickness of the plate. These were plotted (as before) for the beginning of all plates, and the ends of $1 \mathrm{~mm}, 5 \mathrm{~mm}$, and $9 \mathrm{~mm}$ thick plates. The top left plot shows the Blackman pulse source that is applied to the beginning of all plates. The top right plot shows the pulse upon reaching the end of a $1 \mathrm{~mm}$ thick plate. Here it can be seen this is almost identical to the source. The next plot in the bottom left shows the result at the end of a 5 $\mathrm{mm}$ thick plate. The dispersion effects of the guided wave are visible here as it can be seen some energy has been transferred to the trailing lobe, creating an asymmetry in the pulse. This can be observed in Figure 5.4 (c) where the leading lobe is now a lighter blue. The plate is now at sufficient thickness such that some of the higher frequency components of the pulse are travelling at a slower velocity than the lower frequency components, but still propagate as a fundamental symmetric mode. This can be understood by analysing the frequency content of the Blackman pulse shown in Figure 5.2. For a pulse with a centre frequency of $100 \mathrm{kHz}$, the bandwidth of the Blackman pulse extends from 0-200 kHz. This results in a $f d$ product range of 0 to 1 , which by Figure 5.3 corresponds to a group velocity range of approximately $5260 \mathrm{~ms}^{-1}$ to $5400 \mathrm{~ms}^{-1}$. Observing the final plot in the bottom right for the $9 \mathrm{~mm}$ thick plate (an $f d$ product range of 0 to 1.8), the dispersion is more exaggerated, where enough energy travelling at a slower velocity has been transferred to create a second major peak. More energy is now travelling at slower velocities as more minor peaks are now visible. This can be clearly observed in Figure 5.4 (d) where significant energy is trailing the original pulse and the leading lobe contains significantly less energy than before.

\subsection{Single Water Loaded Aluminium Plate}

The free boundary aluminium plate simulations confirmed the dependence of the wave group velocity upon both the incident wave frequency and plate thickness. This was extended by modelling the aluminium plate as immersed in water by loading the aluminium plate with water on both sides. The model used for this simulation is shown in Figure 5.6. Absorbing boundaries were applied to the three boundaries shown to prevent the reflection of any waves incident upon them back into the model. Absorbing boundaries are purely absorbing for normally incident waves but poor absorbers at near grazing incidence (absorbing grazing boundaries can be used for such incidence). However given the angles of incidence of waves 


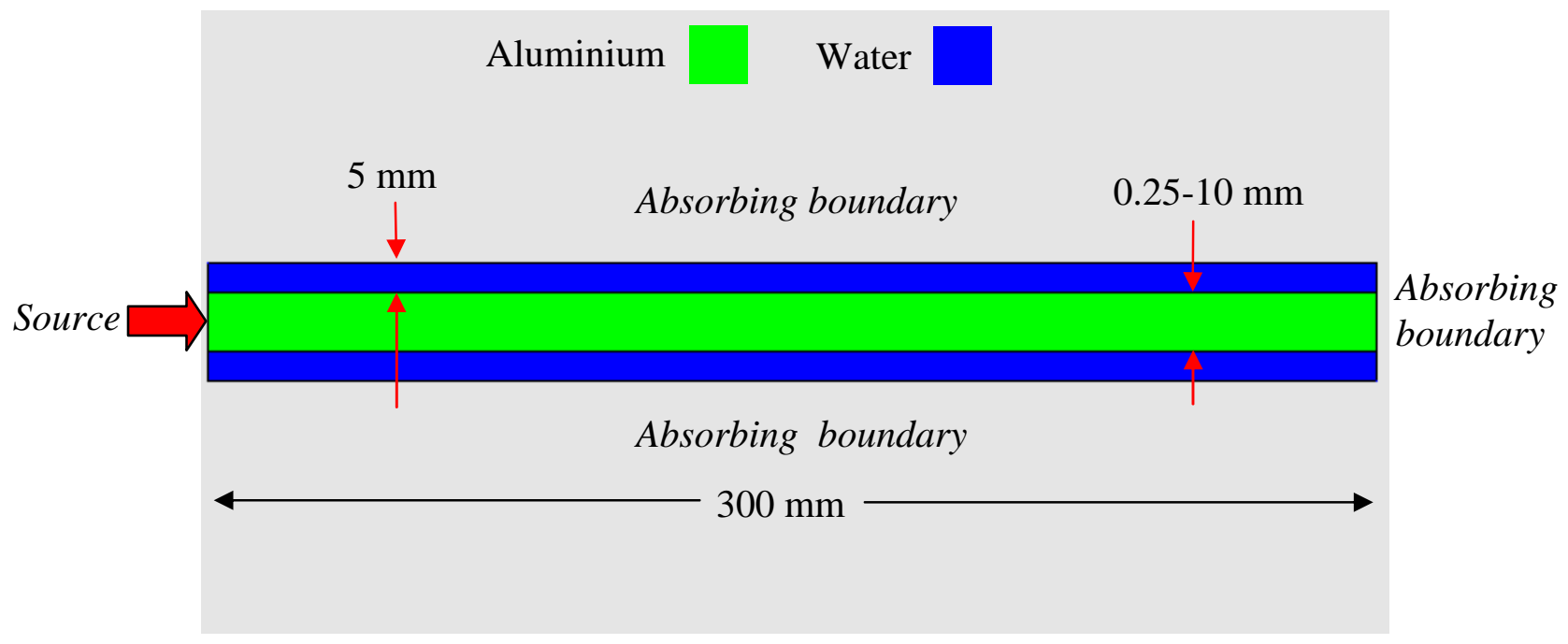

Figure 5.6 - Finite element model of a fluid loaded aluminium plate with boundary conditions shown (dimensions not to scale).

generated in this model were not near grazing incidence absorbing boundaries were appropriate. This reduces the size of the model and computation time. Here the water widths were made 5 $\mathrm{mm}$ so any waves propagating in the water could also be easily observed. A $100 \mathrm{kHz}$ Blackman pulse was applied only to the elements of the aluminium plate on the left boundary as shown above. The length of the model was $300 \mathrm{~mm}$ and the element size used was $62.5 \mu \mathrm{m}$.

Figure 5.7 (a)-(c) shows three images of the pressure in a $5 \mathrm{~mm}$ thick plate, each taken $47 \mu \mathrm{s}$ into the simulation using different pressure scales to reveal several interesting points. Figure 5.7 (a) shows a single waveform propagating in the aluminium plate with a pressure scale just large enough to prevent amplitude saturation of the pulse in the plate. On this scale it appears almost all the energy is confined solely to the aluminium plate.

In Figure 5.7 (b), the pressure scale amplitude has been reduced by a factor of six so that the plate wave amplitude has saturated the scale. Now it can be seen energy does leak from the plate into the surrounding water, since a wake (called a bow wave) can be observed to propagate along with the aluminium plate wave but in the water. The total energy leaked into the water is very small since the pressure scale has been reduced significantly yet the amplitude in the water still appears small. The total amount of energy leaked into the water is dependent upon how far along the plate the wave has propagated. In the observed simulation shot, the total energy leaked into the water is just $0.14 \%$ of the plate wave energy. 


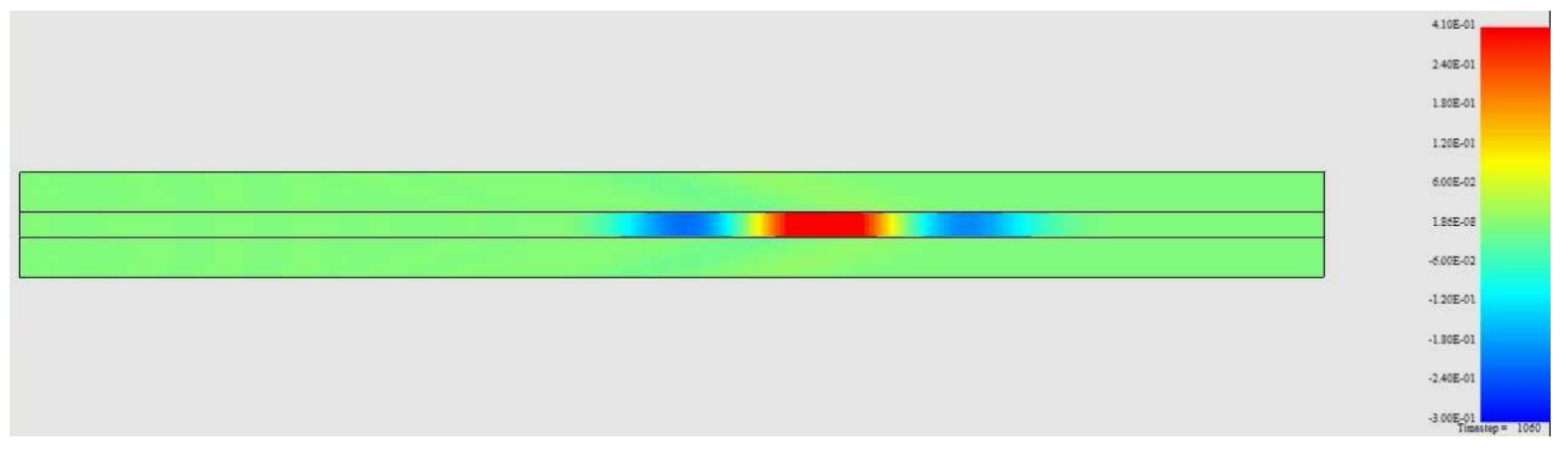

(a)

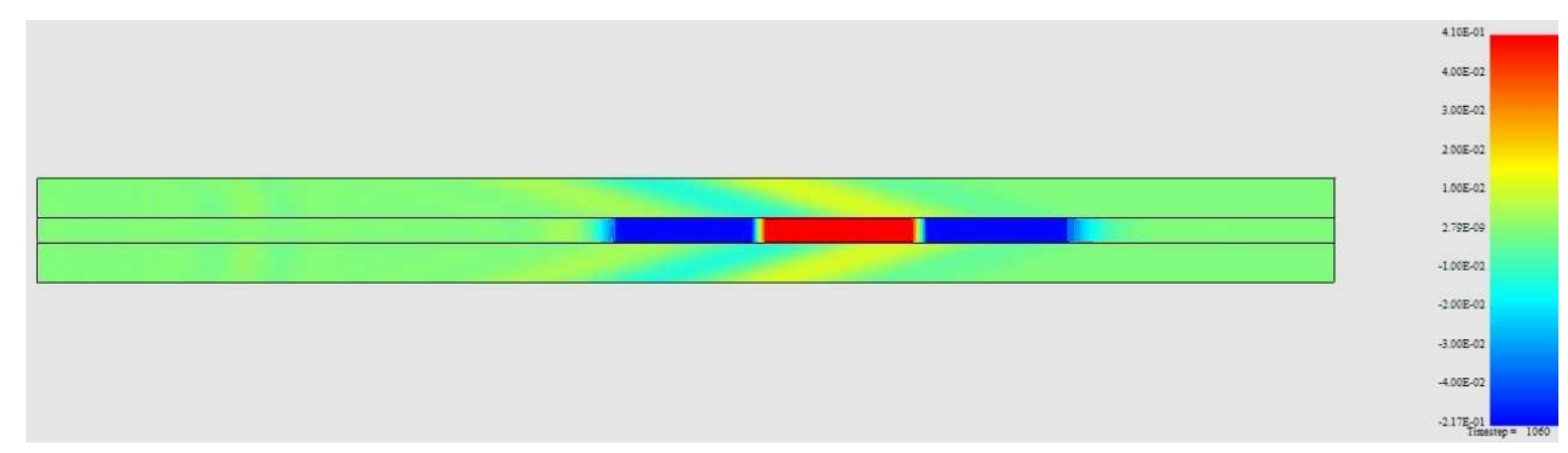

(b)

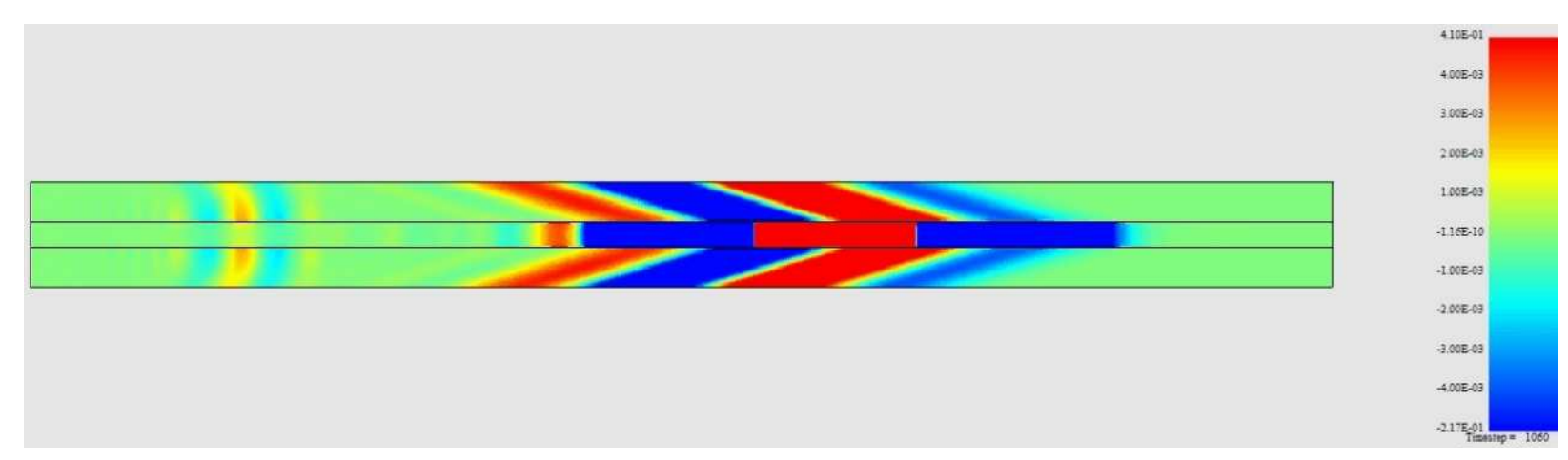

(c)

Figure 5.7 - Water loaded aluminium plate snapshots $47 \mu$ s into the simulation for three different amplitude scales.

By reducing the pressure scale by a further factor of ten Figure 5.7 (c) reveals a saturated plate wave and bow wave, but also identifies an additional slow wave. This wave propagates much slower than the plate wave, appearing to travel at the water velocity and propagating primarily in the water, but with a small amount of energy present in the plate. This wave is the slow mode mentioned in the classic paper by Osbourne and Hart [26]. However, the energy in this 
wave is insignificant when compared with the primary wave. For the distance travelled above, the energy of the slow wave was too small to quantify, at least several orders of magnitude less than the water bow wave. The terms fast wave and slow wave are now used for the wave propagating in the plate and water respectively.

Variation in the fast and slow wave velocities with aluminium plate thickness were investigated using the same method as in the previous section. The plate thickness was varied from 0.25 to $10 \mathrm{~mm}$ and the velocity of both waves measured. The resulting group velocity versus $f d$ product curves for the fast and slow waves are shown in Figure 5.8 and Figure 5.9. The values plotted in Figure 5.8 are in good agreement with those plotted for the free boundary aluminium plate in Figure 5.3. Comparing velocity values at several $f d$ products reveals very little variation between the two curves. The velocity in the limit as the $f d$ product tends to zero is the $\mathrm{S}_{0}$ Lamb mode velocity of $5400 \mathrm{~ms}^{-1}$. Therefore the water loading is not significant enough to have an affect on the fast wave velocity.

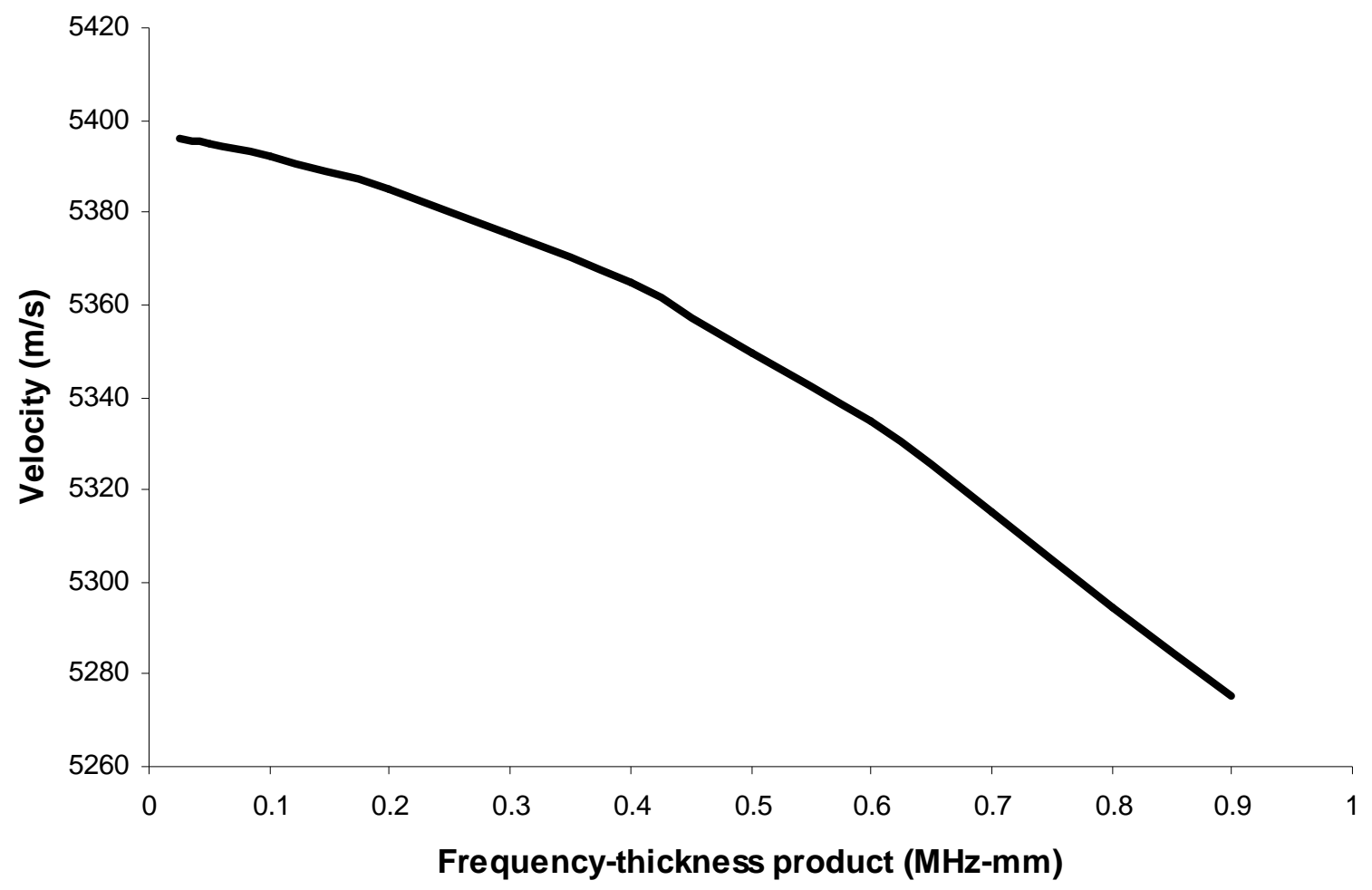

Figure 5.8 - Fast wave velocity in a water loaded aluminium plate with frequency-thickness product. 


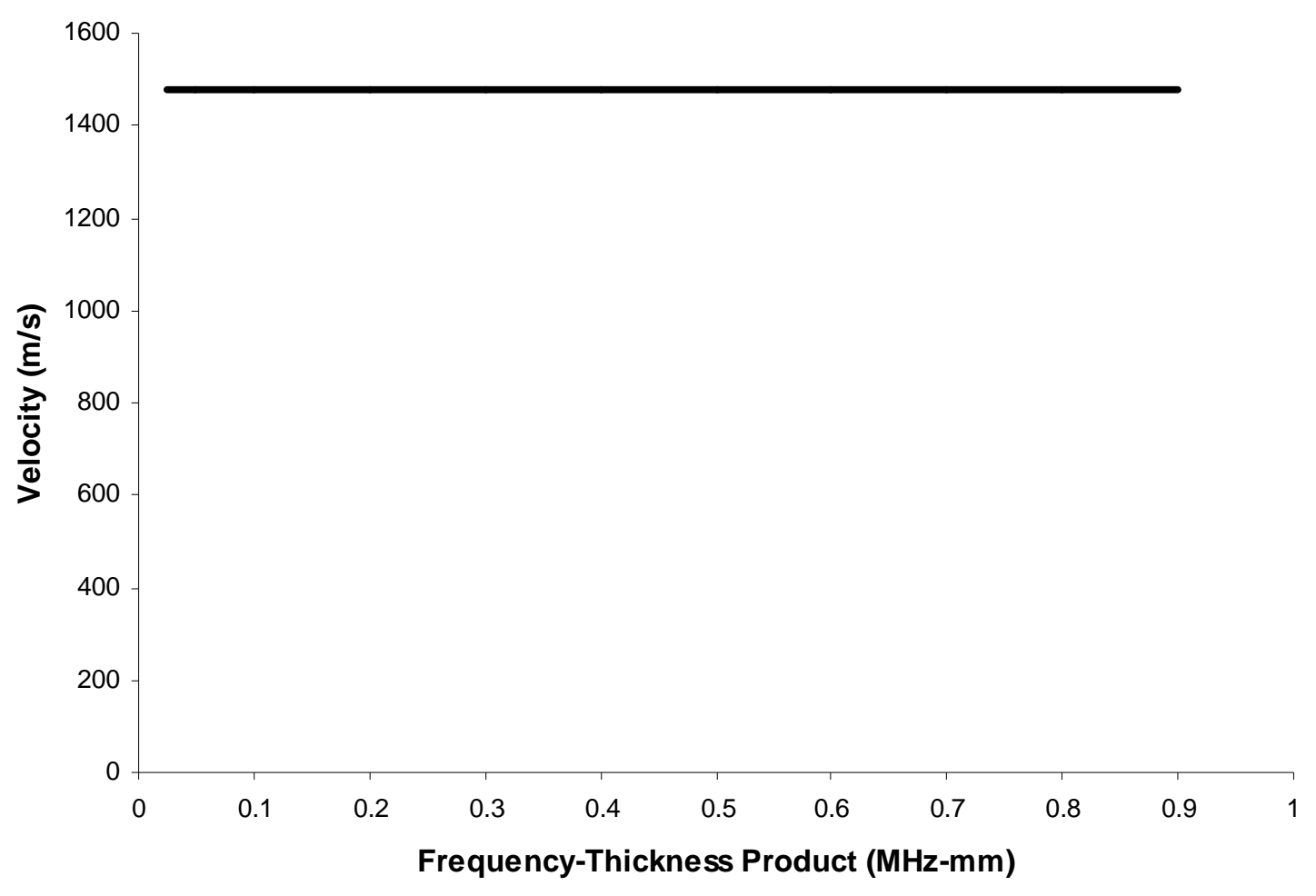

Figure 5.9 - Slow wave velocity in a water loaded aluminium plate with frequency-thickness product.

Figure 5.9 shows the slow wave velocity versus the $f d$ product. This shows a dispersionless velocity characteristic with the wave travelling at a constant velocity of $1480 \mathrm{~ms}^{-1}$ for the plotted $f d$ products.

\subsection{Water Filled Porous Aluminium}

This section extends the previous simulations and studies by modelling multiple aluminium plates (or pillars) separated by water filled pores, creating an ideal porous microstructured material in two dimensions. This layer was then connected to solid aluminium to demonstrate the difference in wave propagation between microstructured and homogeneous media. This simulation was performed for both the excitation of a single aluminium plate and plane wave excitation. The model used and associated boundary conditions for the point source model are shown in Figure 5.10. 


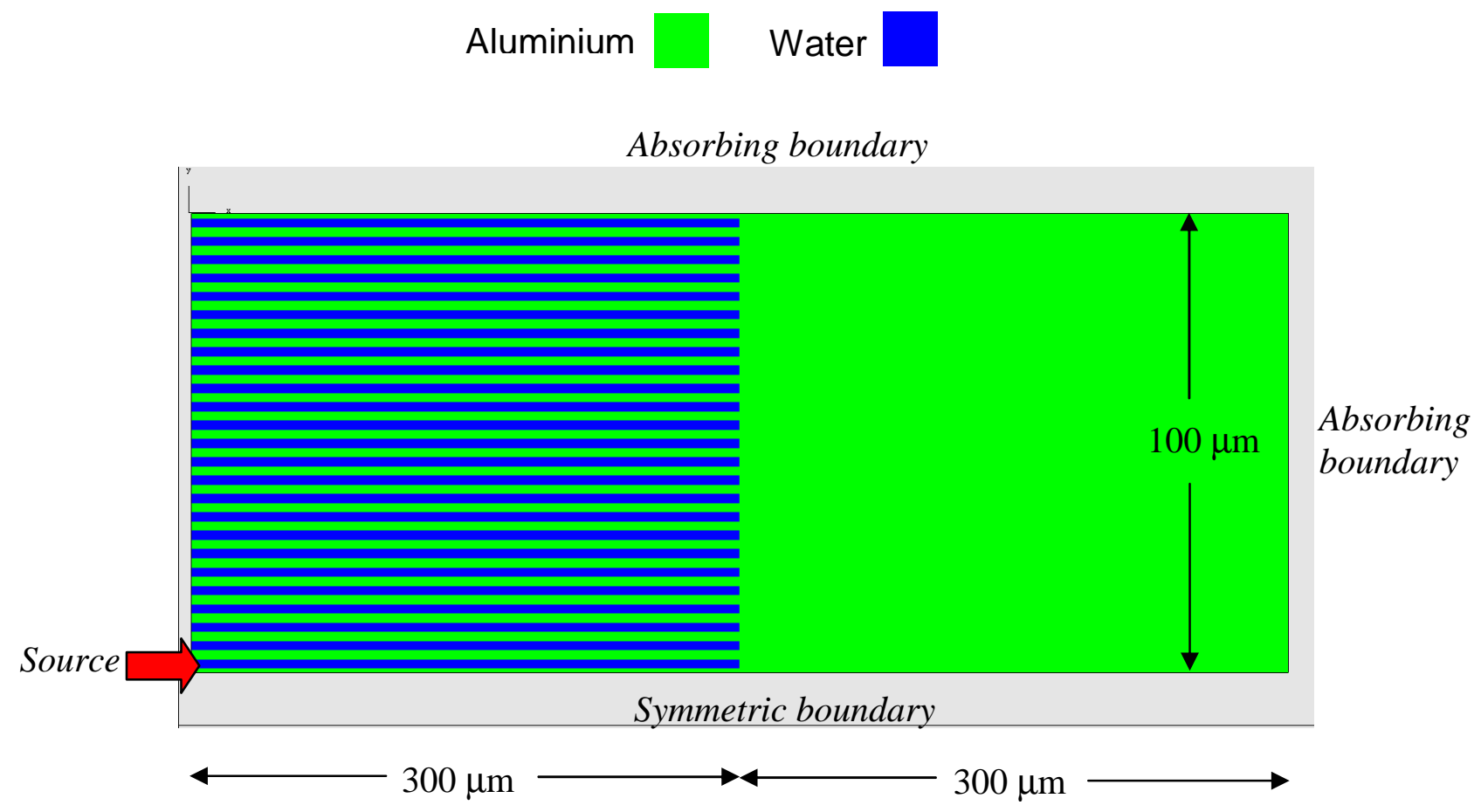

Figure 5.10 - 2D model of a water loaded porous aluminium layer connected to solid aluminium with boundary conditions as shown. The source was excited on the bottom most pillar.

The porous layer consisted of 25 aluminium pillars separated by water filled pores. Both the porous layer and solid aluminium were $300 \mu \mathrm{m}$ in length. The aluminium pillar and water pore widths were $2 \mu \mathrm{m}$. The source was a $50 \mathrm{MHz}$ Blackman pulse, initially incident on the bottom most aluminium pillar as shown in Figure 5.10. A frequency of $50 \mathrm{MHz}$ was used as this is the lowest operating frequency of the transducers used in the experimental work. This pillar is half the size due to the symmetrical boundary condition applied to that boundary. These parameters result in a frequency-thickness product of $0.2 \mathrm{MHz} . \mathrm{mm}$. The model mesh size used was $250 \mu \mathrm{m}, 1 / 60$ th of a wavelength in the water. Figure 5.11 (a)-(c) shows three snapshots of the plate $x$-displacement taken at $0.35 \mu \mathrm{s}, 0.7 \mu \mathrm{s}$, and $1.05 \mu \mathrm{s}$ after the beginning of the simulation.

After $35 \mathrm{~ns}$, the incident wave is travelling in the first aluminium pillar as a single spatially compact wavefront guided along the pillar with a velocity of $5400 \mathrm{~ms}^{-1}$, rather than as the individual $\mathrm{P}$ and $\mathrm{S}$ wavefronts expected for a point source in a homogeneous solid. This fast wave velocity showed similar $f d$ product dependence as observed in the previous section. The close proximity of adjacent pillars facilitates energy transfer through the water pores into these pillars, resulting in the observed wake. 


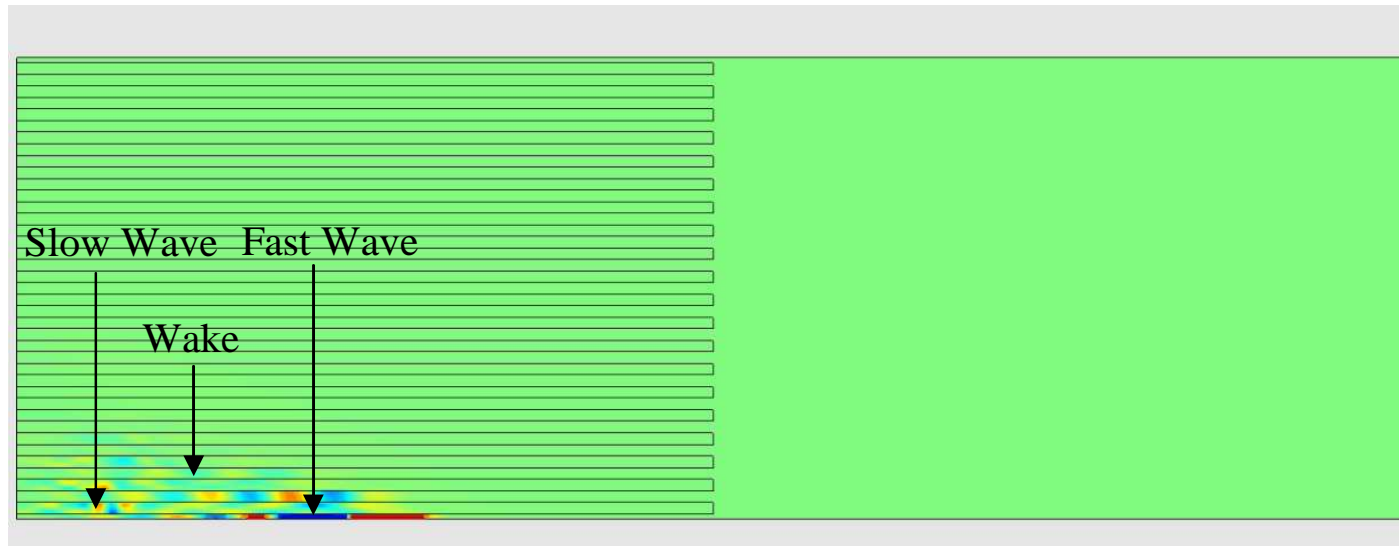

257E-1

800218

(a) $35 \mathrm{~ns}$.

$4.00 \mathrm{E}-18$

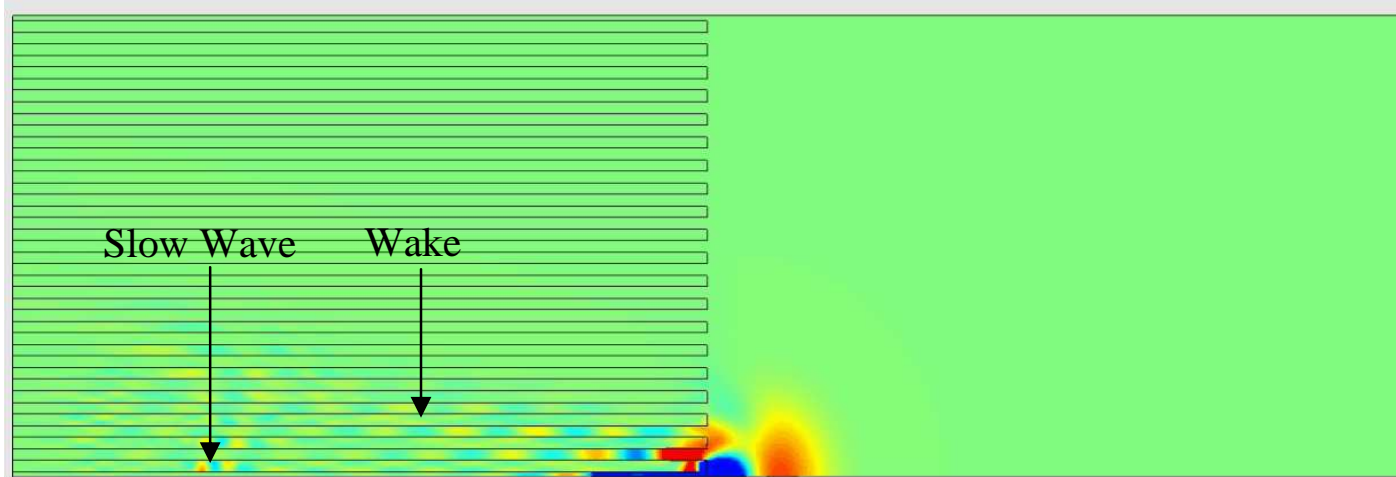

$2000_{-18}$

$6272-25$

200218

$4.00 \mathrm{E}-18$

(b) $70 \mathrm{~ns}$.

6 กUE 18

Interface Wave

Generated Slow

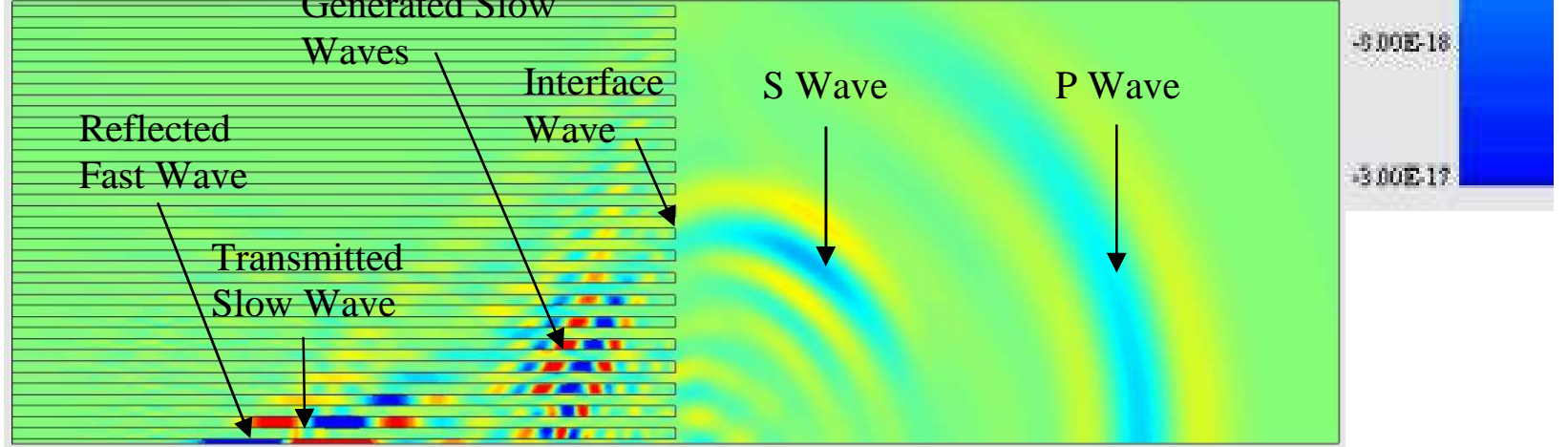

(c) $105 \mathrm{~ns}$.

Figure 5.11 - Simulation of water loaded porous aluminium connected to solid aluminium (a) $35 \mathrm{~ns}$, (b) $70 \mathrm{~ns}$, and (c) $105 \mathrm{~ns}$ after the beginning of the simulation. 
A slow wave can also be observed in the first water channel travelling at the velocity of water of $1480 \mathrm{~ms}^{-1}$. This slow wave velocity was again found to be constant with both frequency and aluminium plate thickness. The amplitude of this wave in this simulation is again much smaller than the fast wave. The $x$-displacement scale is intentionally saturated in order for the slow wave displacement to be visible on this scale. The small energy in the slow wave is due to the fast wave consisting predominantly of longitudinal displacements and little affected by the presence of the water, resulting in very little energy transfer into the water. For a point source the width of the pores influences the amount of energy transferred into adjacent pillars, with more energy transferred for thinner pore widths.

After $70 \mathrm{~ns}$, the wavefront reaches the porous/solid aluminium interface and energy starts to transfer into the solid. Already it can be seen the compact wavefront is now beginning to spread (since the first aluminium pillar acts like a circular source of energy) into the solid aluminium. In this snapshot the slow wave can be observed more clearly, and the wake is spread out over a wider number of pores.

At $105 \mathrm{~ns}$, the model becomes a little more complicated with multiple waves but is easily understood. Upon reaching the material interface and propagating into the solid, the compact fast wave ceases to exist as no longer guided by the porous aluminium pillars the $\mathrm{P}$ and $\mathrm{S}$ components are free to travel at their respective velocities. The individual $\mathrm{P}$ and $\mathrm{S}$ waves spread spherically outwards from the material interface at their respective velocities. The $S$ wave velocity is $3100 \mathrm{~ms}^{-1}$, close to half the $\mathrm{P}$ wave velocity of $6300 \mathrm{~ms}^{-1}$. Figure 5.11 (c) also shows that a reflected fast wave and an interface wave are created. The fast wave propagates to the left at the same velocity as before with an observed wake. This reflected wave is of significance, as this suggests an impedance mismatch between the porous and solid aluminium exists at the interface. Since the acoustic properties of the solid fractions of the porous aluminium layer and the bulk aluminium layer are modelled as the same, the impedance mismatch at the interface is caused by the properties of the propagating waves; this impedance mismatch is further explored in section 5.6. The marked interface wave is coupled to both the $\mathrm{P}$ and $\mathrm{S}$ waves propagating in the solid, and the fast wave in the porous aluminium, with a clear stream of energy connecting them. The influence of the interface wave on the $\mathrm{S}$ wave is the wavefront is not perfectly spherical. The interface wave propagates upwards along the interface and in the process creates new slow waves that propagate to the left in the porous layer. 


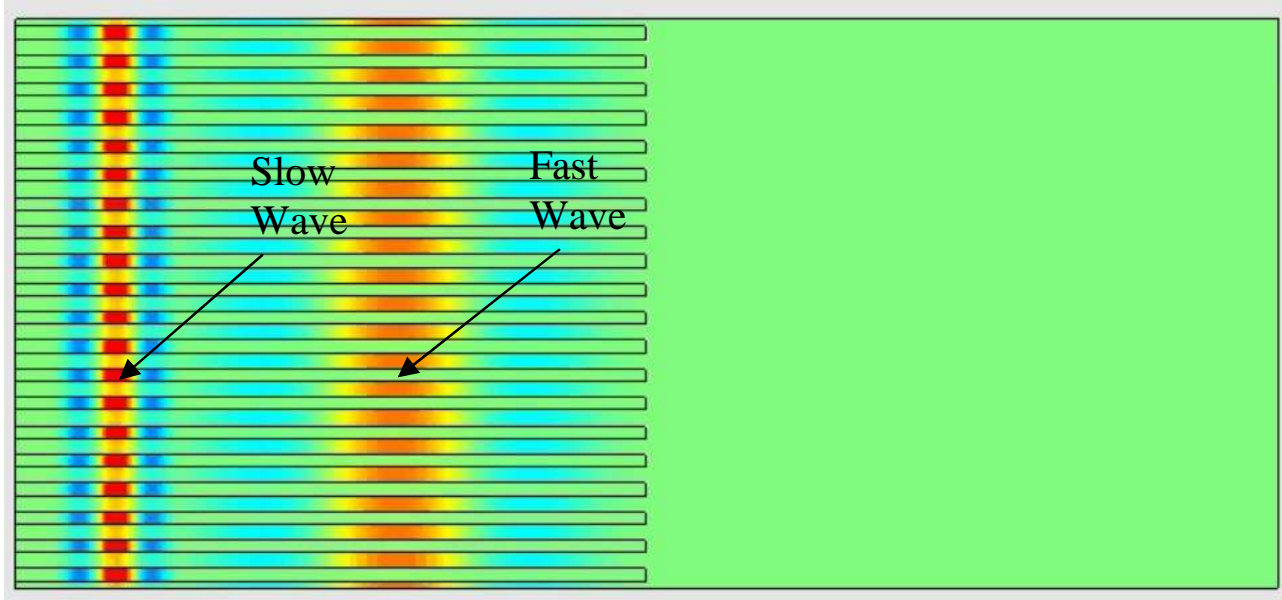

$983 \mathrm{E}-0$

(a) 39 ns.

4 S0E 01
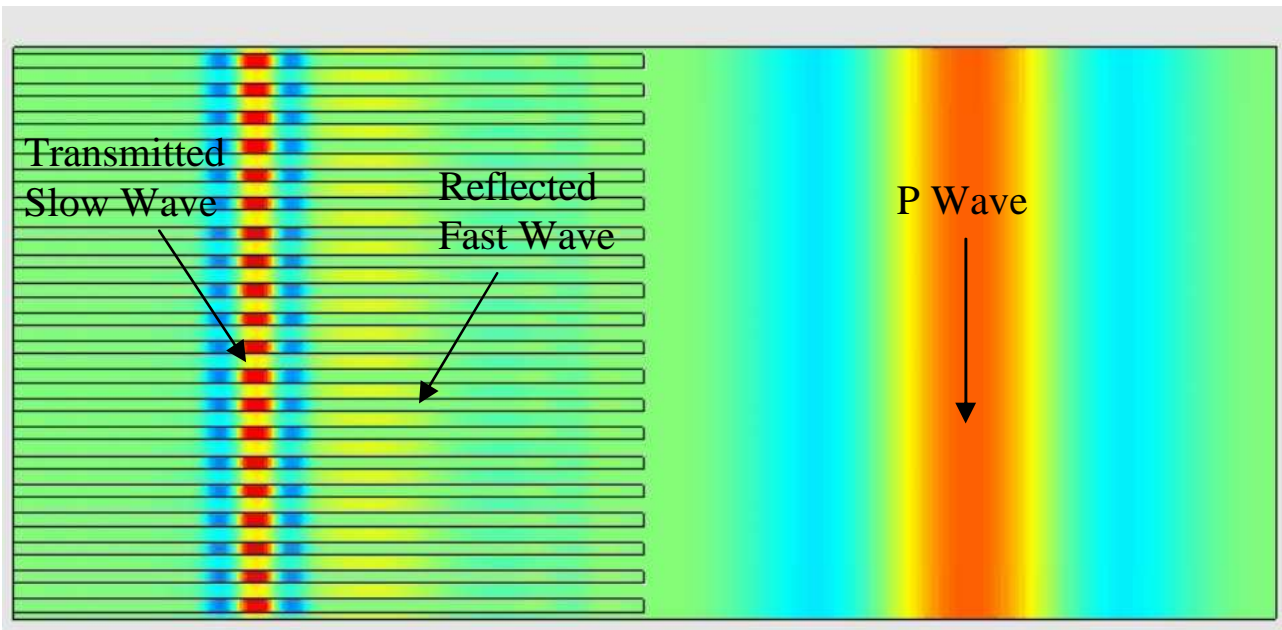

300E-01

$150 \mathrm{E}-01$

$0.00 \mathrm{E}+00$

$150 \mathrm{E} 01$

$300 \mathrm{E}-01$

(b) $71 \mathrm{~ns}$.

$-450 \mathrm{E} 01$

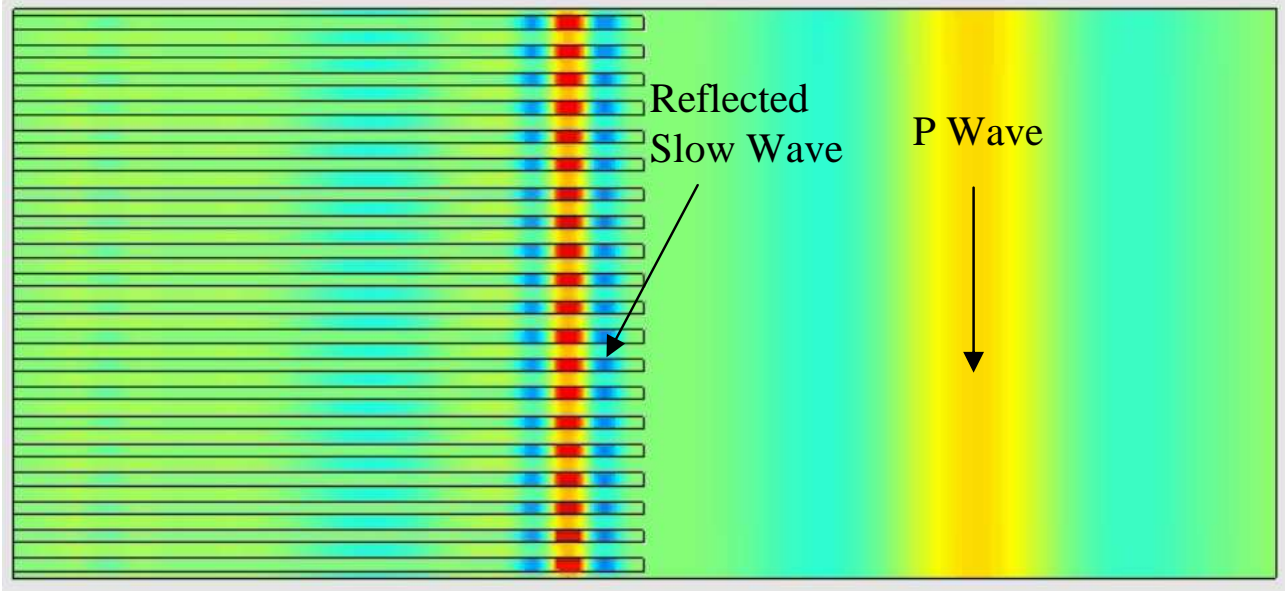

$-6.00 \mathrm{E}-01$

-7 S0E-01

Timestep $=957$

Time $=0.7177 \mathrm{E} 07$

(c) $178 \mathrm{~ns}$

Figure 5.12 - Plane wave excitation of water loaded porous aluminium connected to solid aluminium (a) $39 \mathrm{~ns}$, (b) $71 \mathrm{~ns}$, and (c) $178 \mathrm{~ns}$ after excitation. 
These new slow waves are coupled to the $\mathrm{P}$ wave in the solid as a stream of energy can be seen to connect them. These new slow waves contain significantly more energy than those originally propagating to the right since these have been created by a reflection at the interface, and not created by the small amount of energy leaked between aluminium pillars. The original slow waves propagating to the right can still be observed having almost reached halfway along the porous layer.

For plane wave incidence the result is considerably simpler. The model used for plane wave excitation required only minor changes from the single pillar excitation model. The lengths of both layers were reduced to $200 \mu \mathrm{m}$ and the number of aluminium pillars reduced to 20 . The entire left boundary was then excited and the top boundary modified from absorbing to symmetrical to simulate an infinite plane wave. The bottom and right boundaries were kept the same. Figure 5.12 (a)-(c) shows the plane wave simulation results 39 ns, 71 ns, and 178 ns after excitation. After $39 \mathrm{~ns}$, the slow wave and fast wave are clearly observed propagating at $1480 \mathrm{~ms}^{-1}$ and $5400 \mathrm{~ms}^{-1}$ respectively. In comparison to the single aluminium pillar excitation just discussed, with plane wave excitation significantly more energy propagates in the water filled pores since the entire porous layer face is now excited. In the snapshots, the slow wave appears to contain the same amount of energy as the fast wave. However, not only is the scale saturated in the snapshots, but also the slow wave energy is concentrated over a smaller wavelength. Comparison of the FEM energy integrals of the fast and slow waves reveals the fast wave energy is many times greater than the slow wave. This is explored further below.

After $71 \mathrm{~ns}$ the fast wave has propagated into the solid aluminium where it travels at the $\mathrm{P}$ wave speed in aluminium. This is obvious since there is a noticeable increase in the wavelength. A reflected fast wave of small amplitude can be seen to propagate to the left of the interface generated by the impedance mismatch between the porous and solid aluminium.

Finally, after 178 ns the slow wave has reached the interface, been reflected and now travelling to the left, and transmitted a small amount of energy into the solid aluminium creating a $\mathrm{P}$ wave. This energy transmitted by the slow wave is significantly less than the energy transmitted by the fast wave since the impedance mismatch between the water and the solid aluminium is much greater, resulting in a large reflection coefficient and significant energy reflected back into the layer. 
For plane wave excitation of the layer, the energy transfer is different since the source pulse is now incident on both the water pores and aluminium at the same time. The energy partition between the aluminium and water was analysed with variations in both frequency and aluminium pillar width. Figure 5.13 plots the simulated fast wave energy in the aluminium pillars relative to the slow wave energy in the water pores as an energy partition ratio against variations in wave frequency; a near continuous range of frequencies was used in the generation of this plot. For these measurements the pore and aluminium widths were both $2 \mu \mathrm{m}$, or expressed as a porosity, 0.5 or $50 \%$. Porosity in $2 \mathrm{D}$ is defined as the total pore area divided by the total sample area (use volumes in 3D). As can be observed the energy partition is fairly constant out to $200 \mathrm{MHz}$ showing approximately 11.9 times more energy propagates in the aluminium than in the water for these dimensions. For frequencies greater than $200 \mathrm{MHz}$ the energy ratio begins to slowly decrease with more energy starting to travel in the pores. This change however is only minimal, hence the scale used, decreasing by only 0.3 to 11.4 at $300 \mathrm{MHz}$, the limit of the usable transducer bandwidth of our system. Figure 5.14 examines the fast to slow wave energy ratio dependence upon the pore width relative to the aluminium pillar width, i.e. the layer porosity. This was performed for $50 \mathrm{MHz}$ and $150 \mathrm{MHz}$.

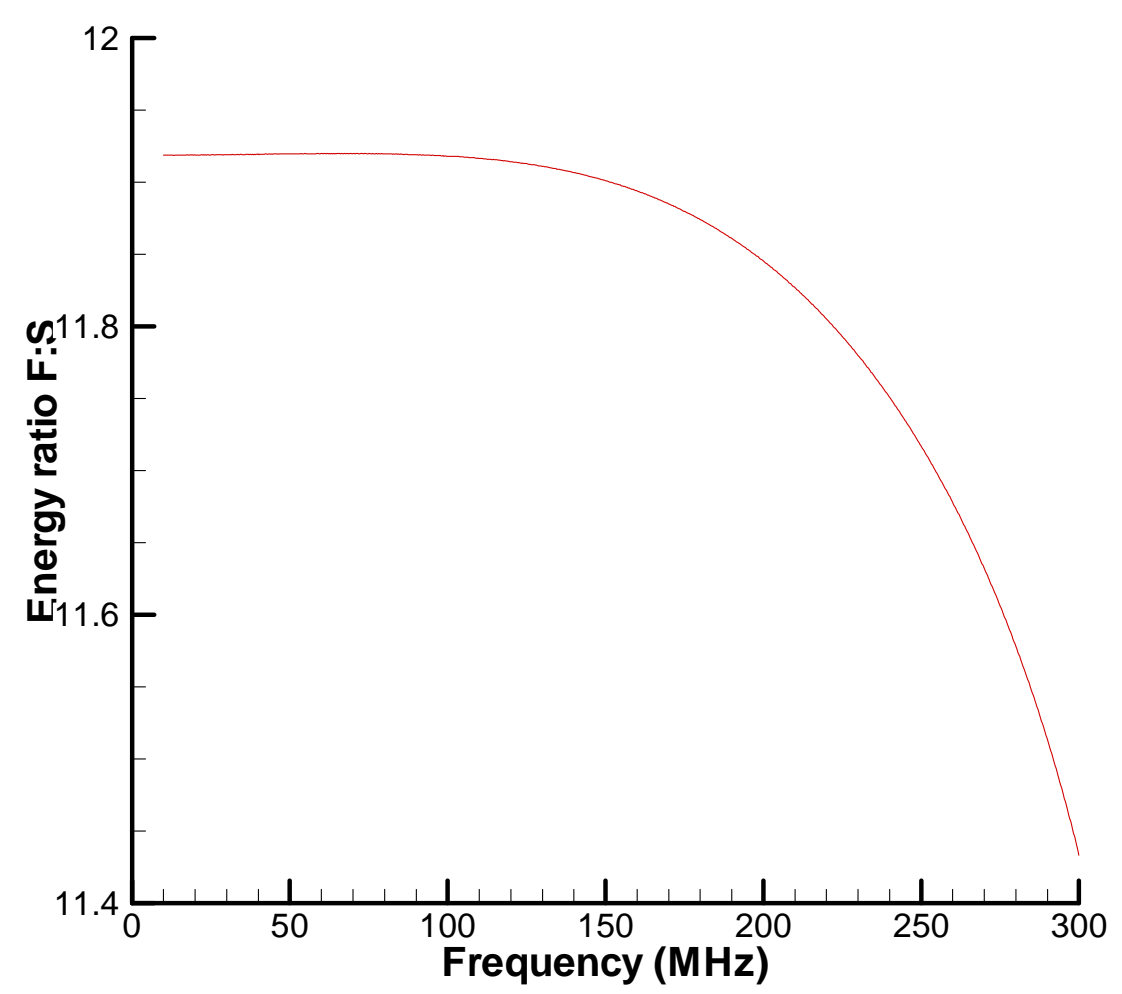

Figure 5.13 - Fast wave to slow wave energy ratio versus frequency for $2 \mu \mathrm{m}$ aluminium pillar and pore widths. 


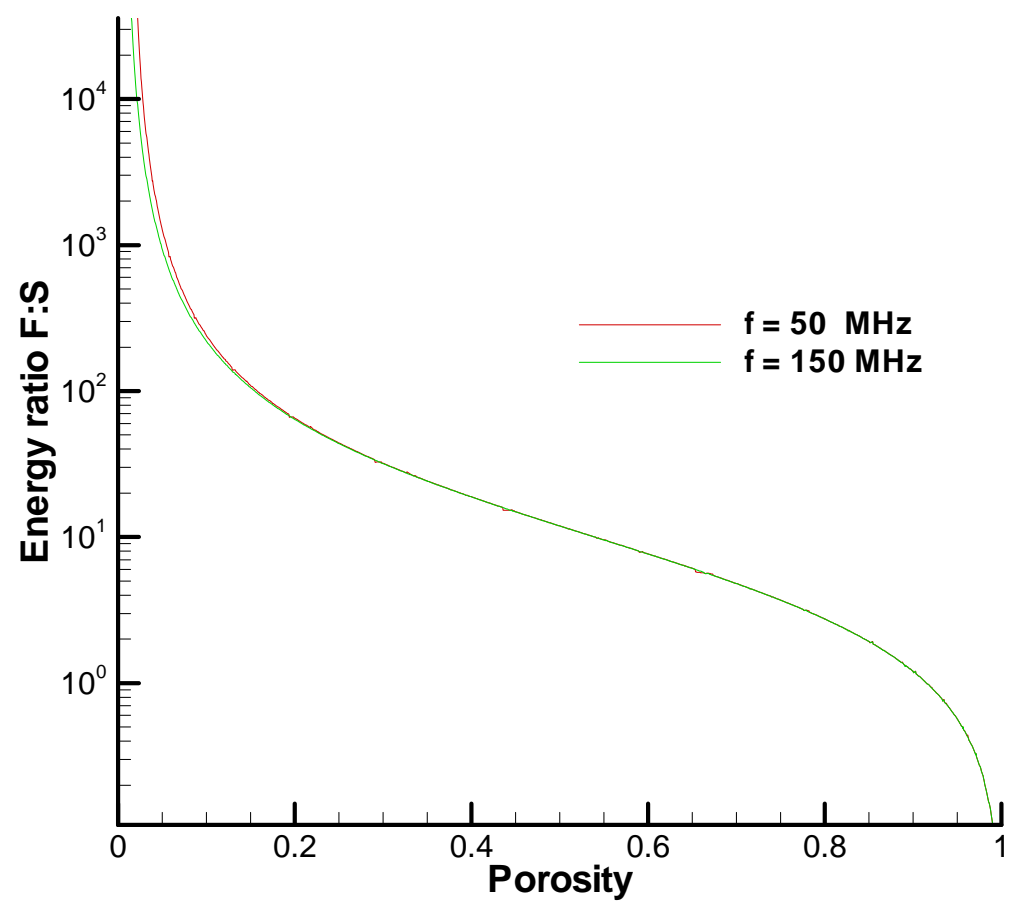

Figure 5.14 - Fast wave to slow wave energy ratio versus porosity at $50 \mathrm{MHz}$ and $150 \mathrm{MHz}$.

Here it can be seen for fixed frequencies with increasing porosity the amount of energy that propagates in the aluminium as a fast wave decreases, and thus the energy content propagating in the water as a slow wave increases. The energy content of the slow wave is however insignificant for low porosities and remains so until approximately 0.9 (or $90 \%$ porosity) where it becomes comparable to the fast wave energy. There is almost no difference between the $50 \mathrm{MHz}$ and $150 \mathrm{MHz}$ traces except for a slightly higher energy ratio at $50 \mathrm{MHz}$ for porosities less than $10 \%$. At the extreme of near $100 \%$ porosity (water medium) the ratio tends to zero since all the energy is now in the water and vice versa for near $0 \%$ porosity.

\subsection{Normalisation and Preservation of Waveforms in Porous Aluminium Layers}

In the previous simulation, for a waveform normally incident on a single aluminium pillar it was observed how a spatially compact wavefront propagates within the porous microstructured layer. This wave, due to the frequency thickness product of the microstructure and incident wave frequency travelled at the fundamental symmetric Lamb mode velocity of $5400 \mathrm{~ms}^{-1}$. This next simulation investigated the propagation behaviour within the microstructured porous layer for the occurrence of non-normal angles of incidence. 


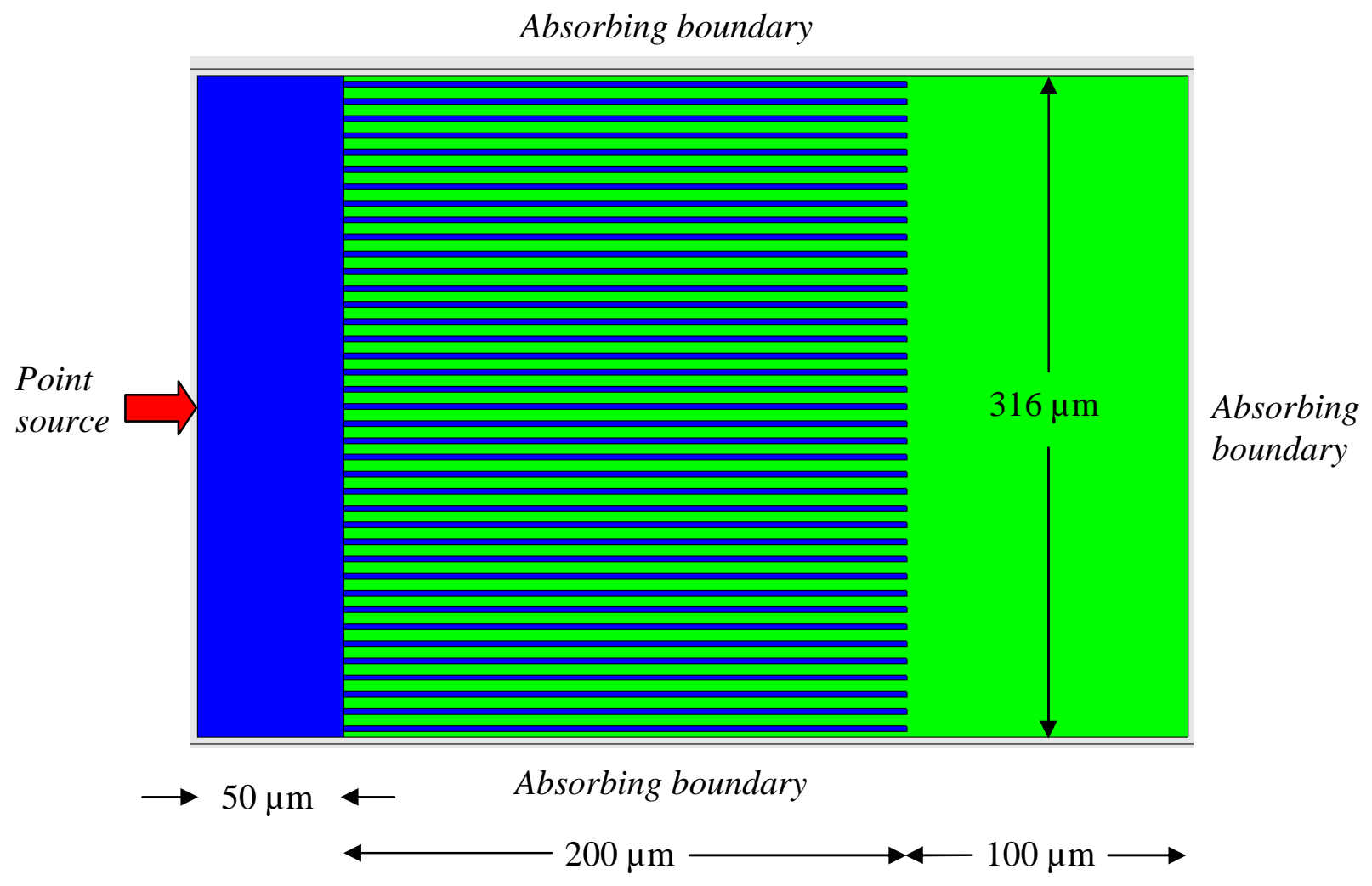

Figure 5.15 - 2D model of a water loaded porous aluminium layer connected to both water and solid aluminium with boundary conditions shown. A single element was excited as shown.

The model structure and boundary conditions are shown in Figure 5.15. The model consisted of a porous aluminium layer with water filled pores, connected to both water on the left and solid aluminium on the right. The porous layer was $200 \mu \mathrm{m}$ in length, the water layer $50 \mu \mathrm{m}$, and the solid aluminium $100 \mu \mathrm{m}$. The porous layer consisted of aluminium pillars of width $4 \mu \mathrm{m}$ separated by $4 \mu \mathrm{m}$ water filled pores. In this model there were 40 aluminium pillars. The boundary conditions were absorbing boundaries on all sides apart from the excitation boundary. The element size of the model was $250 \mathrm{~nm}$. The source used was a $50 \mathrm{MHz}$ Blackman pulse incident on a single element in the centre of the left most boundary. The purpose of using a point source here was to generate a spherical waveform that was incident on the porous layer at all angles of incidence, from zero to ear normal. Figure 5.16 (a)-(c) shows three snapshots taken at three different times during the simulation.

Figure 5.16 (a) is captured $35 \mathrm{~ns}$ after the start of the simulation. As can be observed the waveform generated from the single element excitation was a spherical waveform impinging upon the first interface at all angles of incidence. The wavelength in the water was $30 \mu \mathrm{m}$. 


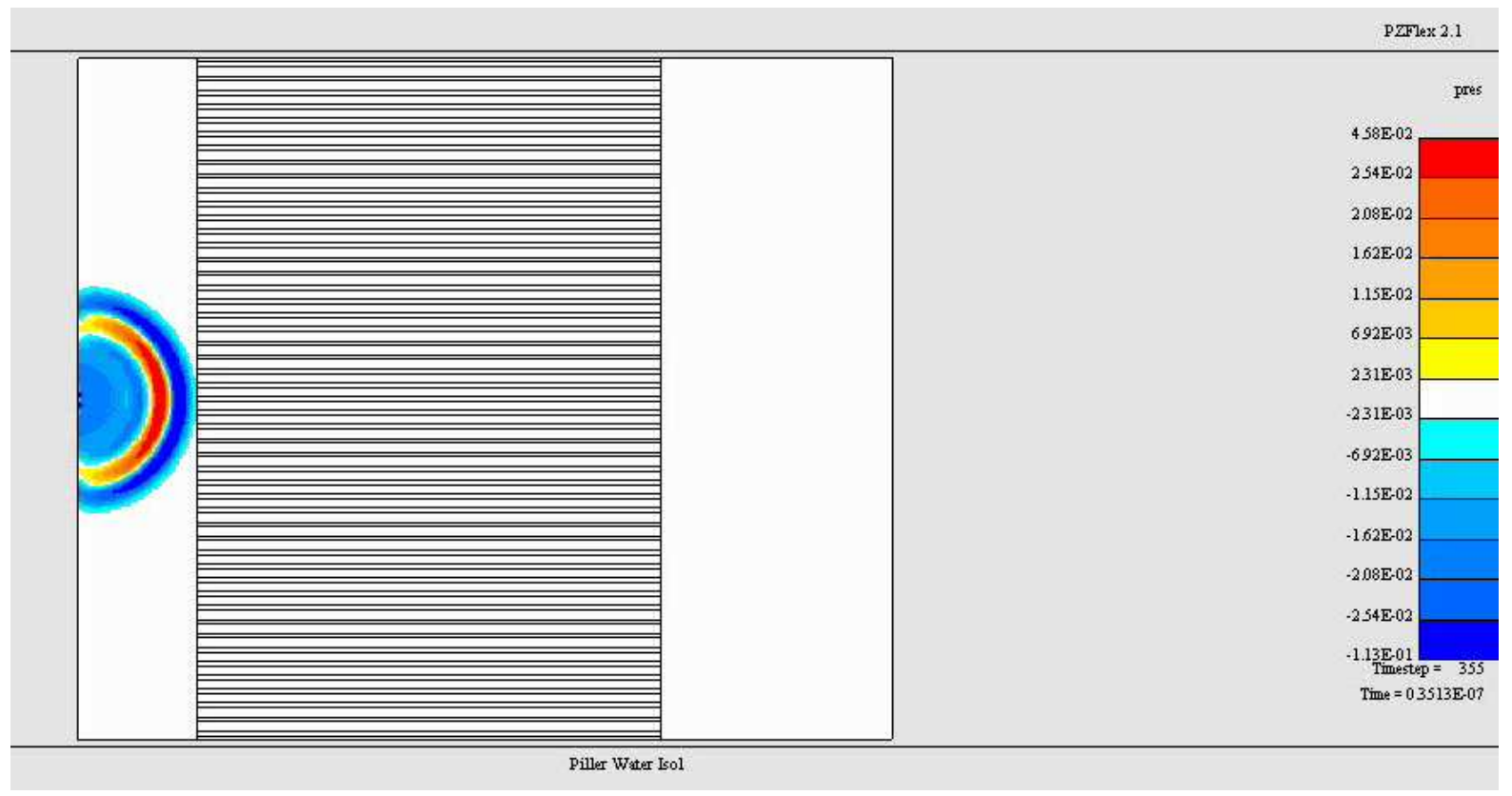

Figure 5.16 (a) - Simulation of spherical wave $35 \mathrm{~ns}$ after the beginning of the simulation.

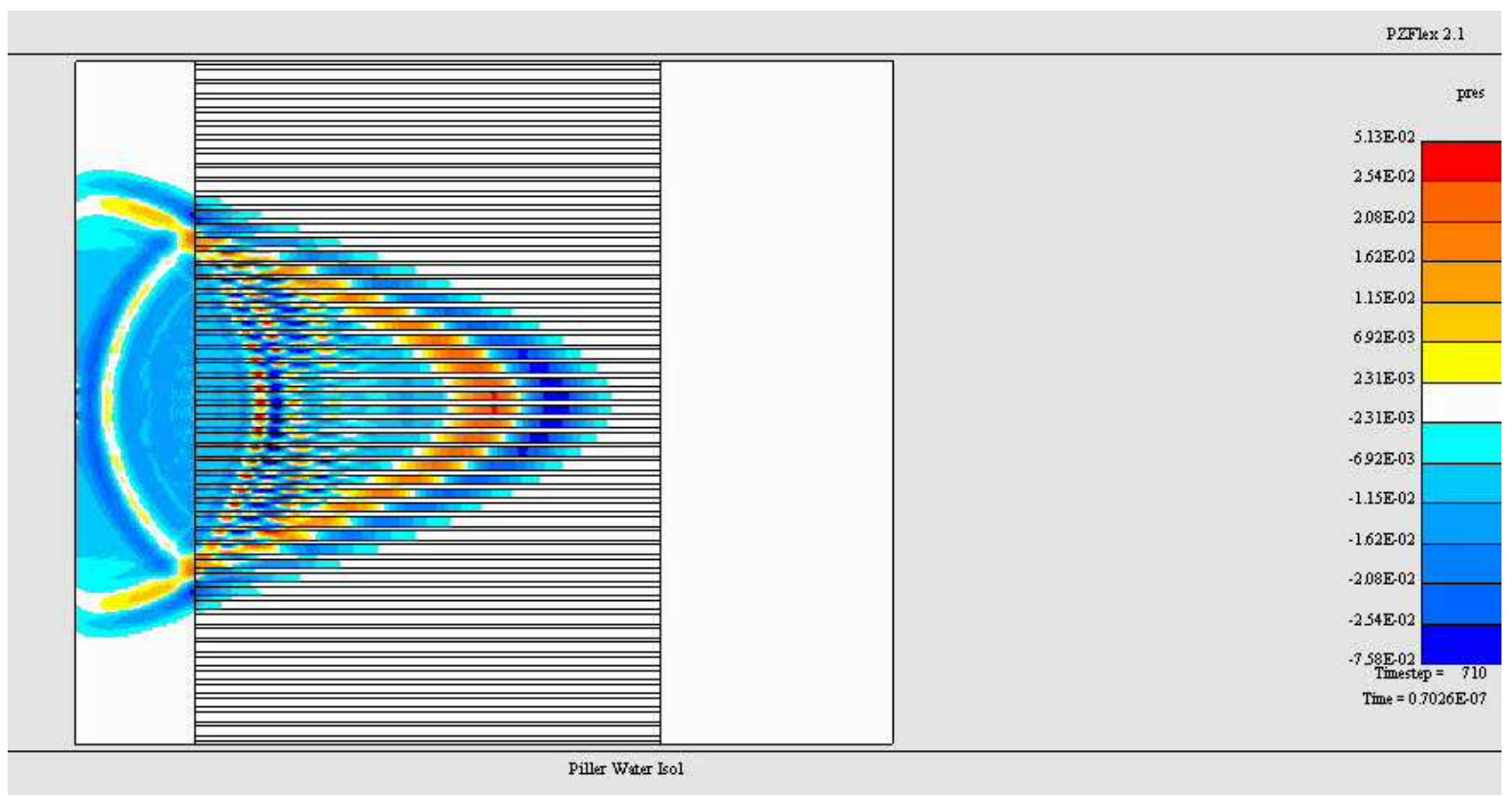

Figure 5.16 (b) - Simulation of spherical wave 70 ns after the beginning of the simulation. 


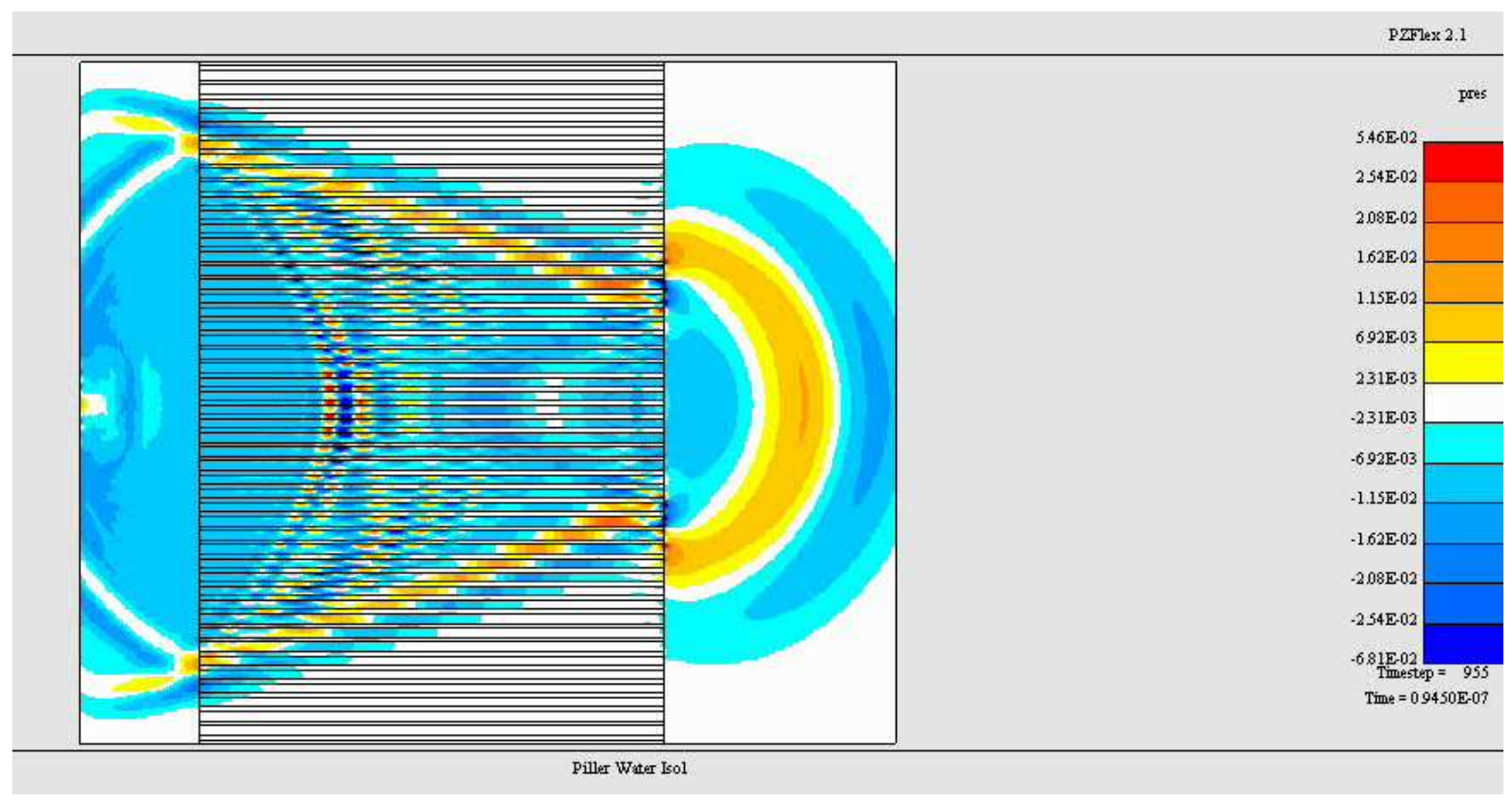

(c) after $90 \mathrm{sec}$

Figure 5.16 - Simulation of spherical wave (a) $35 \mathrm{~ns}$, (b) $70 \mathrm{~ns}$, and (c) $90 \mathrm{~ns}$ after the beginning of the simulation.

Upon reaching the water/porous layer interface, Figure 5.16 (b) shows a snapshot of the waveform $70 \mathrm{~ns}$ after the beginning, now propagating inside the porous layer. A reflected waveform can be observed to propagate back into the water, due to the impedance mismatch between the porous aluminium and water. Inside the porous layer, two waveforms are now observed - a fast wave and a slow wave. Measuring the velocity of the waves using the same technique as in previous simulations, the velocity of the fast wave is measured again to be $5400 \mathrm{~ms}^{-1}$ and the slow wave $1480 \mathrm{~ms}^{-1}$. An interesting point is the entire spherical wave shape is preserved upon incidence of the porous layer. For homogeneous aluminium, using equation (2.19) from section 2.3.2, critical angles of $14^{\circ}$ and $29^{\circ}$ exist for the $\mathrm{P}$ and $\mathrm{S}$ waves respectively. For angles of incidence greater than or equal to these, the respective waves travel along the interface and do not propagate into the medium.

In contrast to this, it can be seen from Figure 5.16 (b) that the porous layer allows transmission for all angles of incidence, normalising the incident waveform. At the porous layer interface Snell's law no longer applies since it is only applicable at homogenous media interfaces. Instead guided wave propagation occurs in the microstructure, eliminating the occurrence of first and second critical angles. Also, during transmission through this layer, the wave does not 
continue to spread spherically as occurs in homogeneous media, but rather maintains the same shape of the wave upon entry into the porous layer. This result has significant implications as the information in many imaging systems is obtained from the shape of a received waveform.

The final snapshot Figure 5.16 (c) is taken 90 ns after the beginning of the simulation. This shows the wave exiting the porous layer and entering the solid aluminium. A small almost unnoticeable waveform is reflected at the porous layer/solid aluminium interface, implying an impedance mismatch between the porous layer and solid aluminium. Once in the solid aluminium, no longer constrained by the porous layer microstructure the wave spreads spherically outwards, exiting the layer with the same waveshape of that upon entering the layer.

\subsection{Acoustic Impedance of Free Boundary Aluminium Plate}

In the previous two simulations, reflections at the porous aluminium/solid aluminium interface were observed. These implied the porous aluminium possessed an acoustic impedance different from that of the solid aluminium. In this simulation the variation in the percentage energy reflected (or energy reflection coefficient) at a single aluminium plate/solid aluminium interface as a function of plate thickness was investigated. Figure 5.17 shows the structure of the model used with boundary conditions. The model consisted of an aluminium plate $260 \mathrm{~mm}$ in length that widened into a solid aluminium block at the end of the plate.

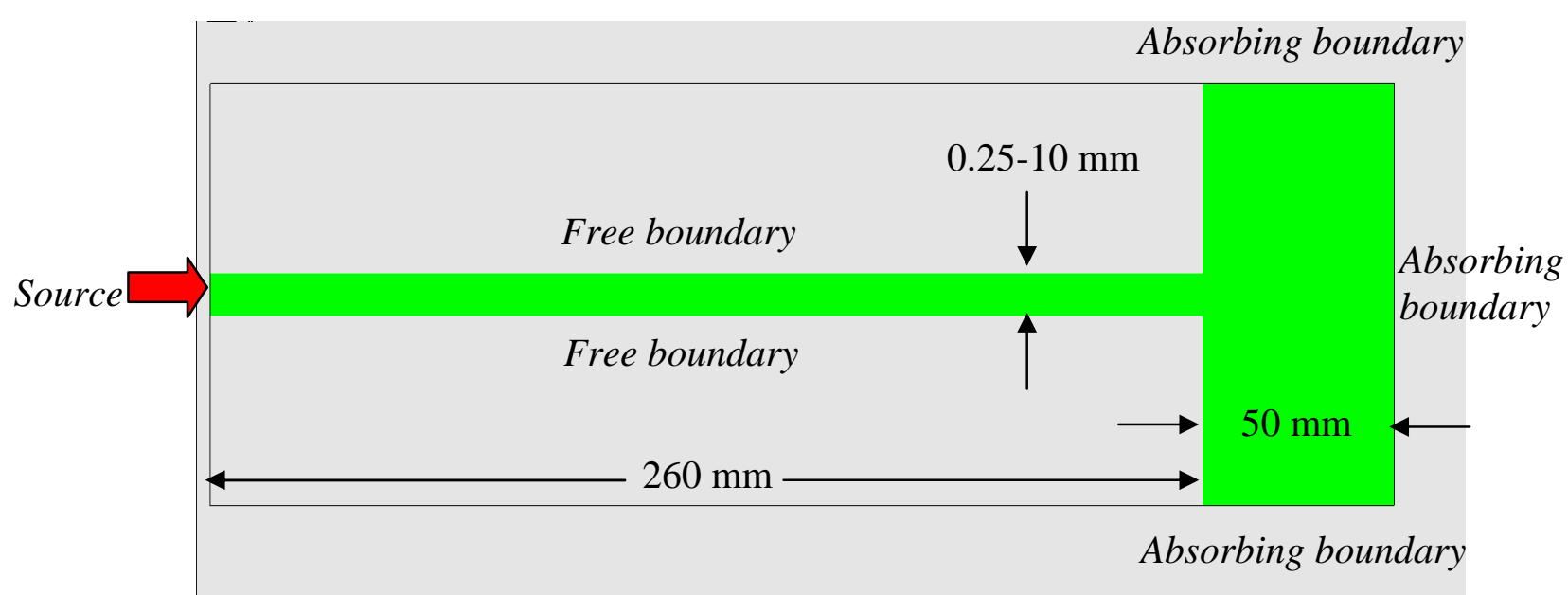

Figure 5.17 - 2D model of a single aluminium plate connected to solid aluminium with boundary conditions shown. 
The dimensions of the large solid block were not critical as it was only used to create the impedance mismatch between the plate and the aluminium solid. The only requirement was that it was wide enough so that bulk wave propagation occurred in the solid. The length of the solid was arbitrary since an absorbing boundary condition was applied to the right most boundary. The source was a $100 \mathrm{kHz}$ Blackman pulse incident on the entire left most boundary, creating a plane wave. This choice of frequency is arbitrary provided the plate dimensions are of the right thickness relative to a wavelength in order to observe reflectivity changes. The thickness of the plate was varied from $250 \mu \mathrm{m}$ to $10 \mathrm{~mm}$, resulting in a frequency-thickness product range of 0.025 to $1 \mathrm{MHz} . \mathrm{mm}$. An element size of $50 \mu \mathrm{m}$ was used to provide adequate spatial resolution for the smallest plate thickness. The simulation was run for $100 \mu \mathrm{s}$, providing enough time for a wave to travel to the end of the plate and a reflected wave to propagate halfway back. The energy content of this wave needs to be measured to determine the reflection coefficient at the interface.

Figure 5.18 (a)-(c) shows three energy integral and instantaneous power plots with time, calculated for a vertical line of nodes stretching the thickness of the plate, at several points along the plate length for a $1 \mathrm{~mm}$ thick plate. Figure 5.18 (a) shows the energy integral and instantaneous power plots at the beginning of the aluminium plate, (b) halfway along the aluminium plate, and (c) beginning of the widened aluminium. The energy plot shows the net energy that has propagated through the line of nodes with time. Any energy propagating through a line is recorded as positive if it is right going (travelling away from the source), and negative if it is left going (reflected back towards the source). This can be observed in Figure 5.18 (b). The lower trace shows the instantaneous power with time through the line of nodes defined halfway along the plate where both a positive transmitted wave and a negative reflected wave are observed. The energy integral above that reflects this where the energy reaches a maximum then drops as the reflected wave passes through the plane in the opposite direction. To calculate the energy reflection coefficient the total original energy and total reflected energy were required to be found. The original energy was found using the energy integral result of Figure 5.18 (a). The energy integral result of Figure 5.18 (b) was the original energy less the reflected energy. By taking the energy integral result of Figure 5.18 (b) and subtracting that from the energy integral of Figure 5.18 (a) the reflected energy could be calculated. Taking this result as a fraction of the original energy gives the energy reflection coefficient $R$. The resulting reflection coefficient variations with frequency-thickness product are shown in Figure 5.19. 


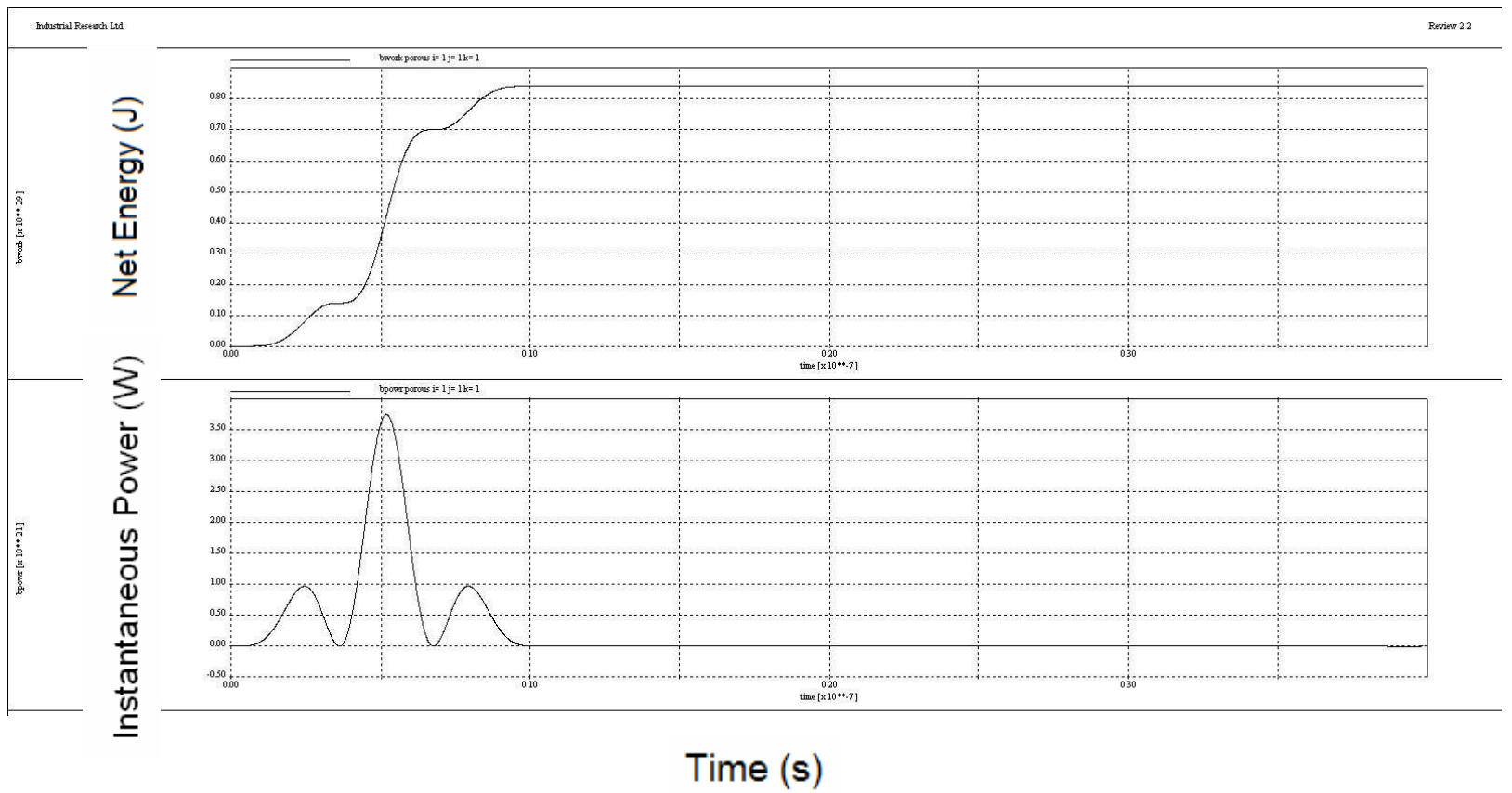

Figure 5.18 (a) - Energy integral and instantaneous power plots for a $100 \mathrm{kHz}$ wave in an aluminium plate of $1 \mathrm{~mm}$ thickness, recorded at the beginning of the aluminium plate.

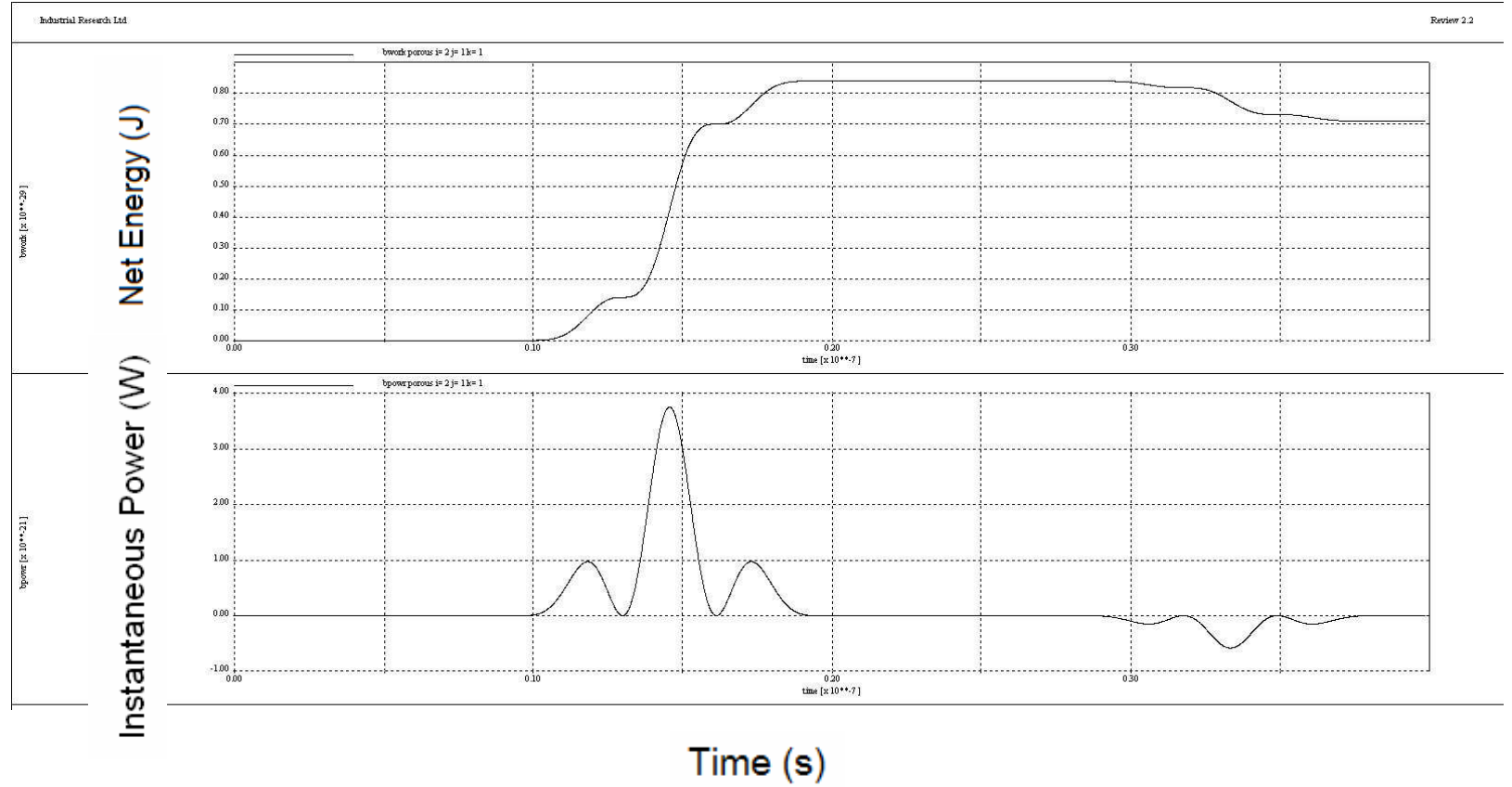

Figure 5.18 (b) - Energy integral and instantaneous power plots for a $100 \mathrm{kHz}$ wave in an aluminium plate of $1 \mathrm{~mm}$ thickness, recorded halfway along the aluminium plate. 


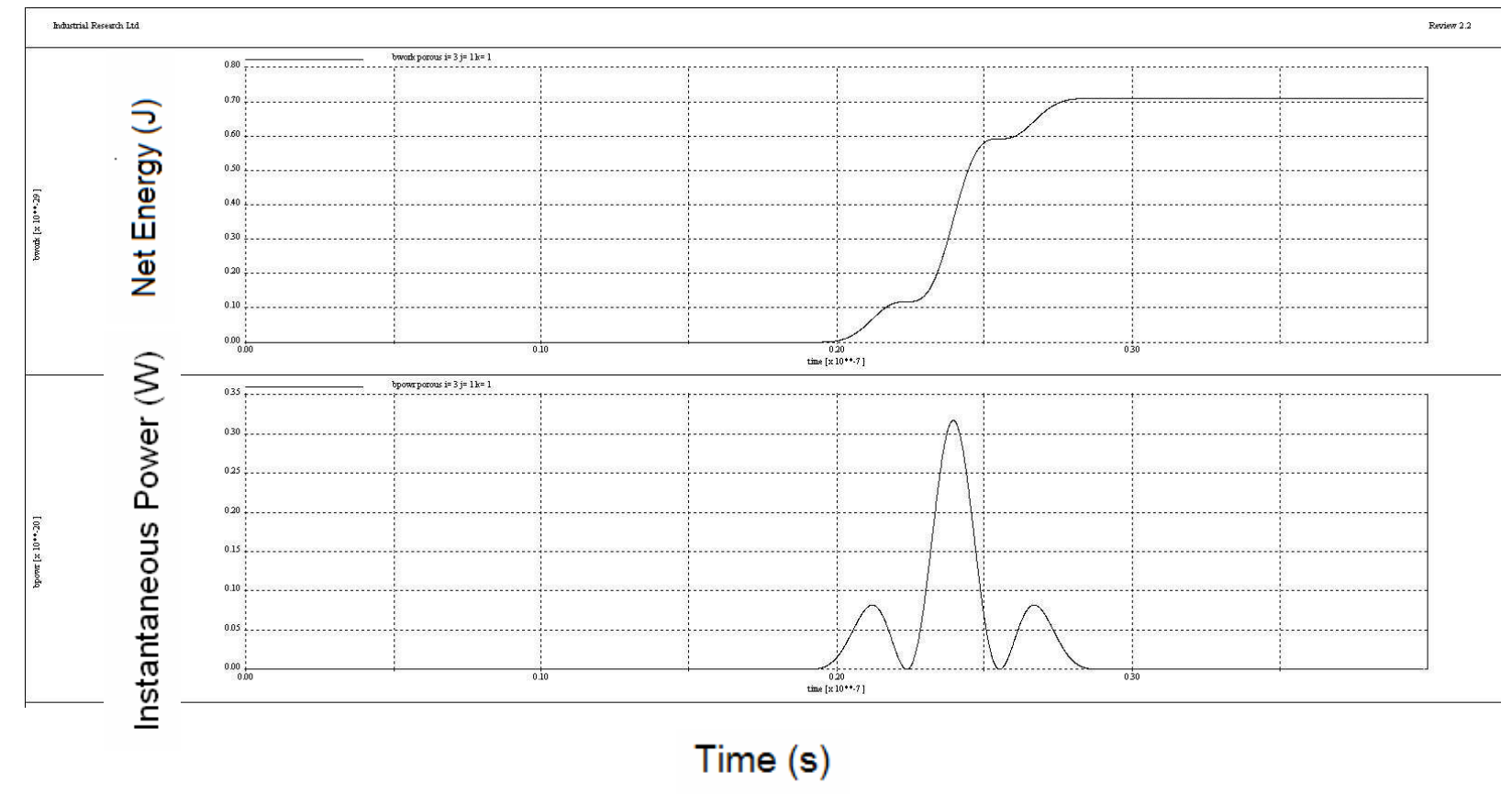

(c) Beginning of widened aluminium solid

Figure 5.18 - Energy integral and instantaneous power plots for a $100 \mathrm{kHz}$ wave in an aluminium plate of $1 \mathrm{~mm}$ thickness. Snapshots are recorded at (a) the beginning of the aluminium plate, (b) halfway along the aluminium plate, and (c) at the beginning of the widened aluminium solid.

Observing Figure 5.19 the reflection coefficient at the plate/solid interface is greatest when the frequency-thickness product is smallest. As the frequency-thickness product is increased the aluminium plate becomes wider, reducing the difference between the plate and the widened solid, reducing the reflectivity. With an $f d$ product of $1 \mathrm{MHz} . \mathrm{mm}$, the reflection coefficient is approximately just $5 \%$. Equations (2.13) and (2.16) can be combined to given an expression for the unknown plate acoustic impedance $Z_{1}$.

$$
Z_{1}=Z_{2}\left(\frac{1-\sqrt{R}}{1+\sqrt{R}}\right)
$$

where $R$ is the energy reflection coefficient. Using (5.2), the known solid aluminium acoustic impedance of $17 \mathrm{MRayl}$, and the calculated energy reflection coefficients, the variations in plate acoustic impedance is plotted against $f d$ product in Figure 5.20. 


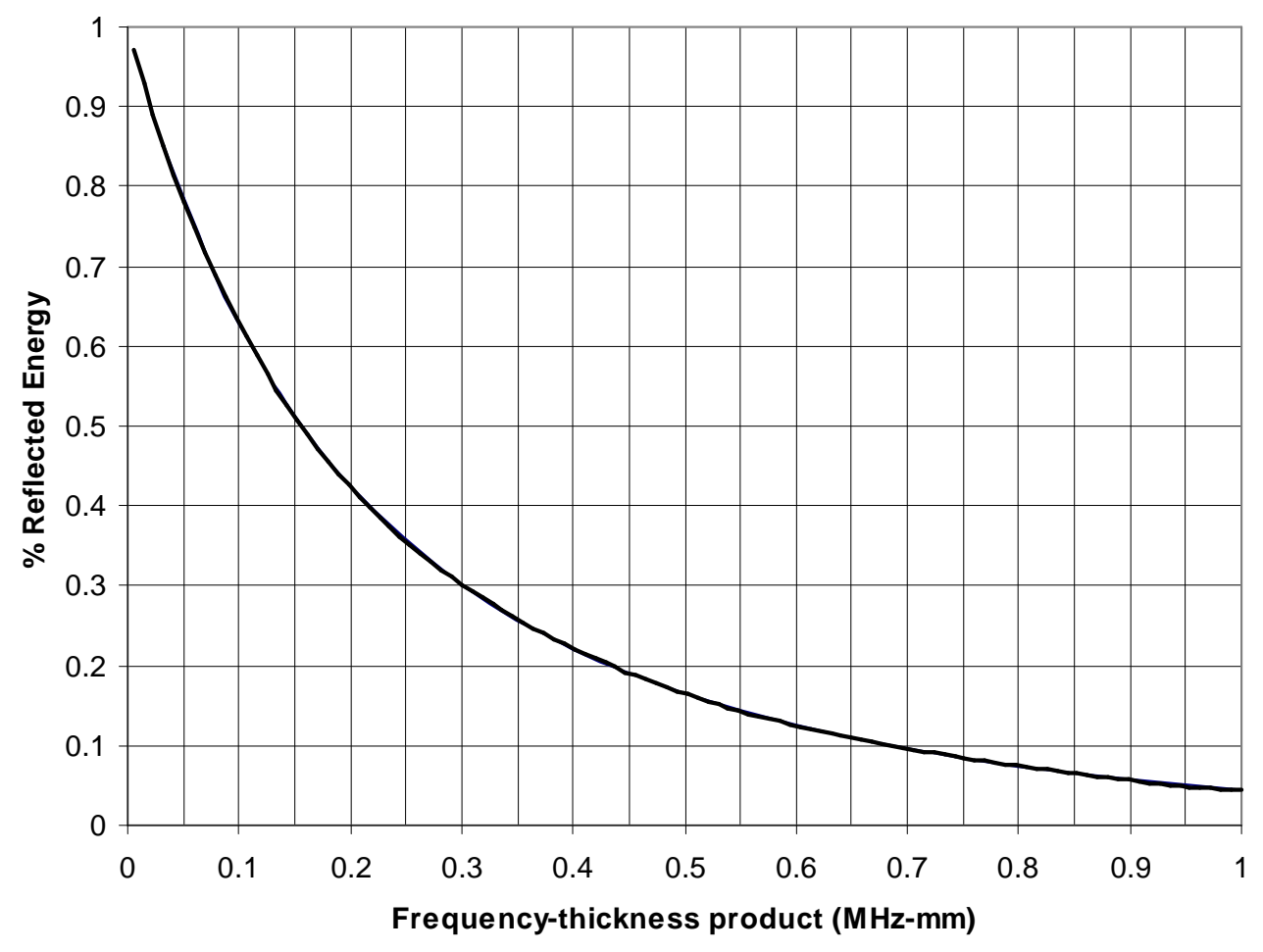

Figure 5.19 - Percentage energy reflected from aluminium plate/solid interface with $f d$ product.

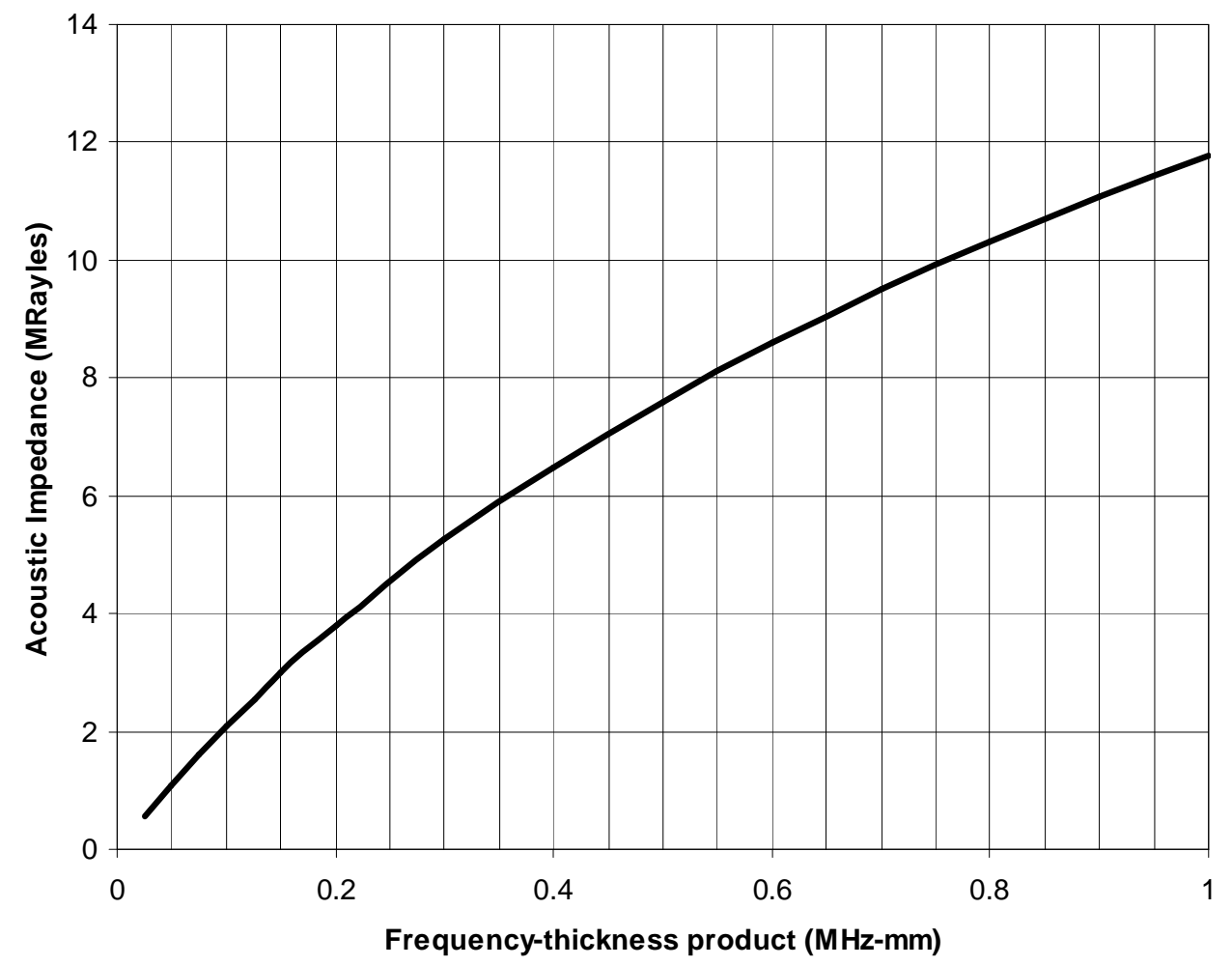

Figure 5.20 - Aluminium plate acoustic impedance versus $f d$ product. 


\subsection{Rectangular and Triangular Architecture Models of PAA Samples}

The 2D modelling performed revealed several points of significant interest

- The velocities in the aluminium plates of all of the simulations were dependant upon the incident wave frequency and aluminium plate/pillar thickness.

- Water loading of the porous aluminium pores caused small amounts of energy to leak to adjacent aluminium pillars, the extent of which was dependent upon the sample porosity.

- For plane wave excitation the porosity of the porous layer determined the energy partition between the water filled pores and aluminium plates.

- In addition to the $S_{0}$ like Lamb mode in the aluminium, a slow wave propagated in the water channel with the $\mathrm{P}$ wave velocity of water.

- Reducing the aluminium plate thickness resulted in an increase in the acoustic impedance mismatch between the solid and porous aluminium layers.

- A microstructured porous layer normalised incident waveforms, allowing reception for angles of incidence well beyond the critical angles that exist at an interface between two homogeneous media.

Whilst the above information was useful, simulations were performed on 3D porous microstructures more representative of the fabricated PAA samples. These 3D models provided a greater insight into what was expected in the experimental work. Models performed in 3D require more elements and calculations leading to increased computation times.

\subsubsection{Triangular and Rectangular Models}

The type 1 PAA samples show a typical hexagonal closely packed (HCP) structure. The type 2 and type 3 PAA samples are however quite different, not exhibiting an obvious HCP structure. Two different architectures were therefore modelled to investigate the influence of architecture on the observed wave guiding phenomena. These were called the rectangular model and triangular model. The rectangular model was used to reflect the main structural properties of the type 2 and type 3 samples since they display similar pore/pore wall architectures, and the triangular model was used to reflect the type $1 \mathrm{HCP}$ like structure. Whilst the respective models incorporate the structural characteristics of the PAA samples, they are not intended to be an exact simulation of the PAA samples. 


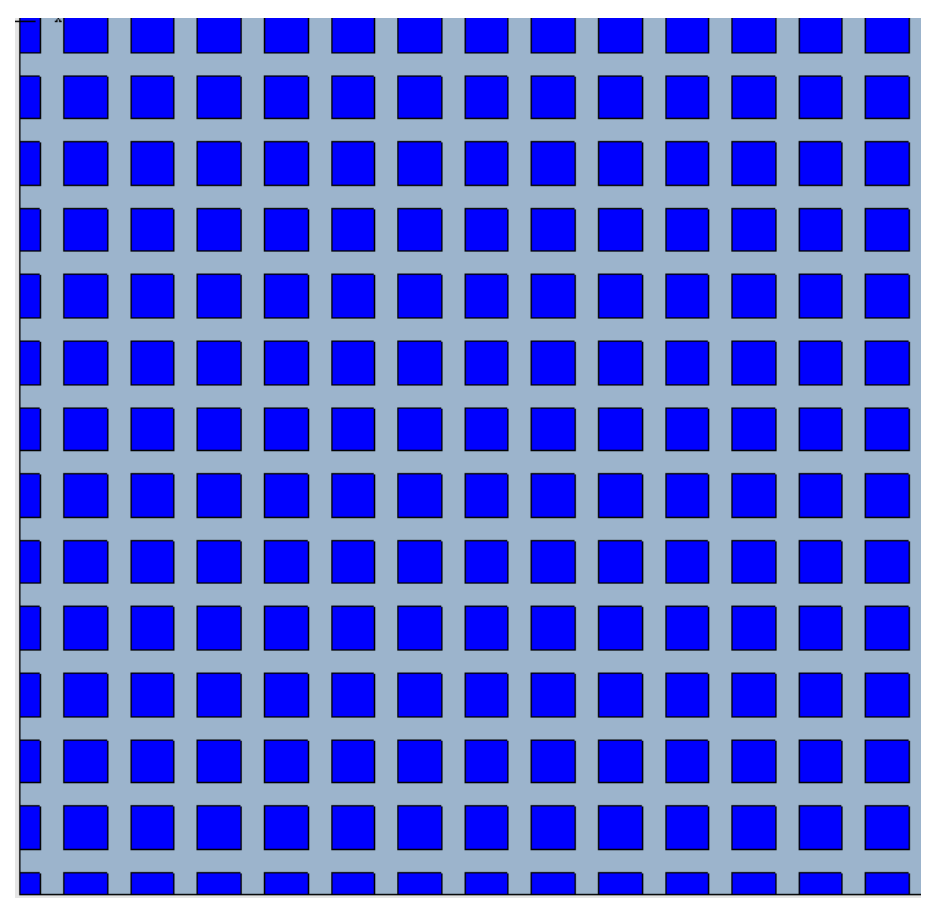

Figure 5.21 - Rectangular model horizontal cross section used to model the type 2 and type 3 PAA sample architectures.

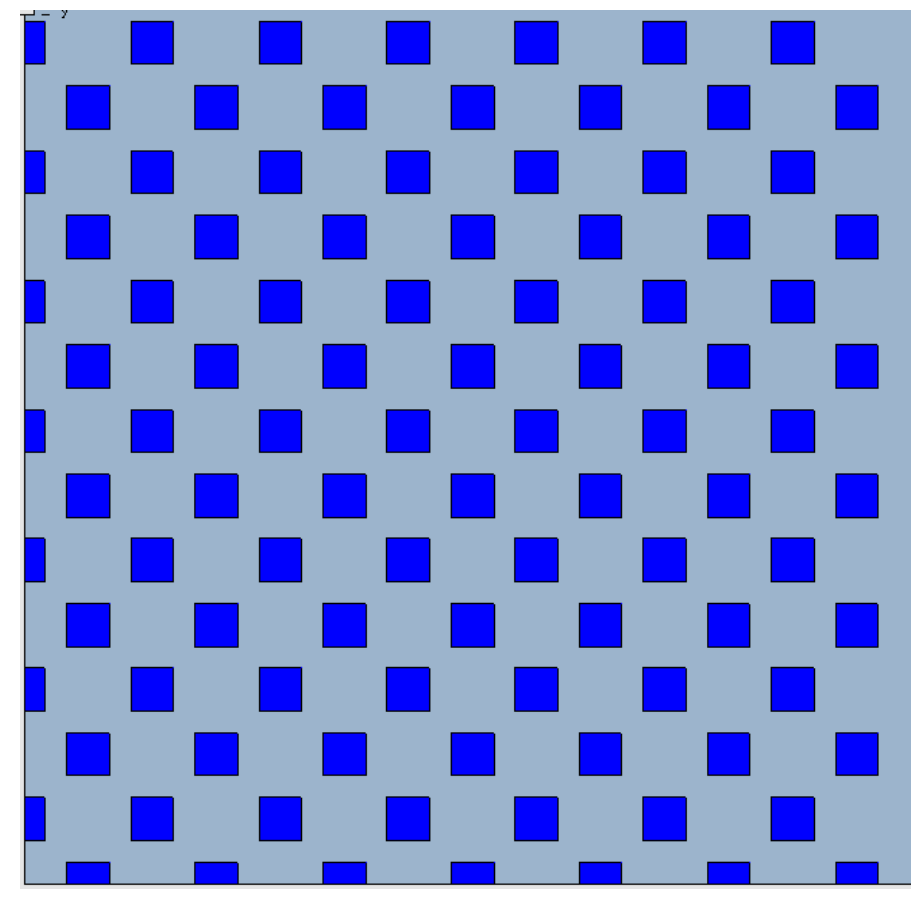

Figure 5.22 - Triangular model horizontal cross section used to model the type 1 PAA sample architecture. 
The wave phenomena observed however provided a good indication for what was expected when experimental measurements were performed on the PAA samples. Horizontal cross sections of the rectangular and triangular models are shown in Figure 5.21 and Figure 5.22 respectively. Blue represents the sample pores, and grey the aluminium pore wall structure. The rectangular model simulates the type 2 and type 3 samples with regularly spaced square pores in a grid separated from each other by aluminium pore walls of uniform thickness. From SEM images the pores of the type 2 and type 3 samples were not rectangular, as consistent in shape, or as well arranged as the grid in Figure 5.21. However the pore size and aluminium pore wall thicknesses were very consistent as simulated in this model.

2D modelling showed the pore size and solid thickness to be the important characteristics determining sample velocity and acoustic impedance. Pores were modelled as squares to reduce the number of elements and thus computation time. SEM images of the type 1 samples showed the pores were arranged in a face centred structure, significantly different from the type 2 and type 3 samples. To simulate this, pores were arranged in a triangular arrangement relative to the surrounding pores. Comparison of the two models shows the rectangular model aluminium content was simulated as many aluminium plates separating the pores, whereas in the triangular model the aluminium becomes more like bars as the aluminium content is reduced.

The cross sections of each model were followed through $100 \mu \mathrm{m}$ in length (the $z$-axis), creating highly collinear porous 3D structures. Pores are assumed void unless stated otherwise. The porous layers were attached to a solid aluminium layer creating an impedance mismatch in order to measure the porous sample reflectivity. Fixing the pore size at $500 \mathrm{~nm}$, simulations were performed for aluminium plate thicknesses in the range 50-4000 nm - a porosity range of $0-80 \%$ for the rectangular samples, and a porosity range of 0-45 \% for the triangular model. This upper porosity limit for the triangular model comes about since as the inter-pore separation is reduced, the pores will eventually come into contact preventing continuous coupling between the aluminium.

By applying symmetry, the two models were reduced to those shown in Figure 5.23 and Figure 5.24, reducing computation time significantly. These reduced models contain all of the structure of Figure 5.21 and Figure 5.22, however with a fraction of the computation time to run the model. The dimension $s$ is the pore separation distance, and $\mathrm{p}$ the rectangular pore width. 


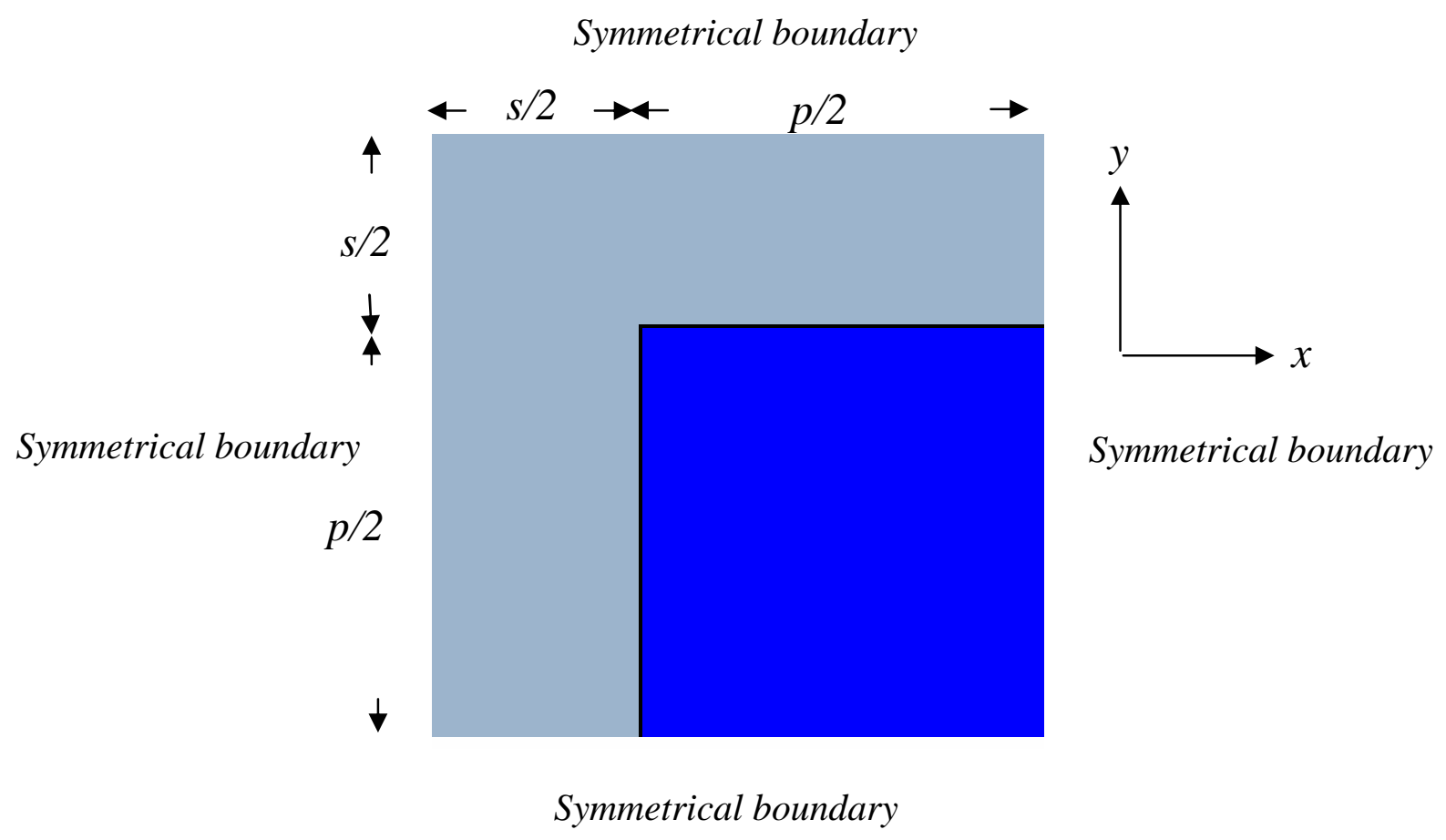

Figure 5.23 - Symmetrically reduced rectangular model with symmetrical boundaries.

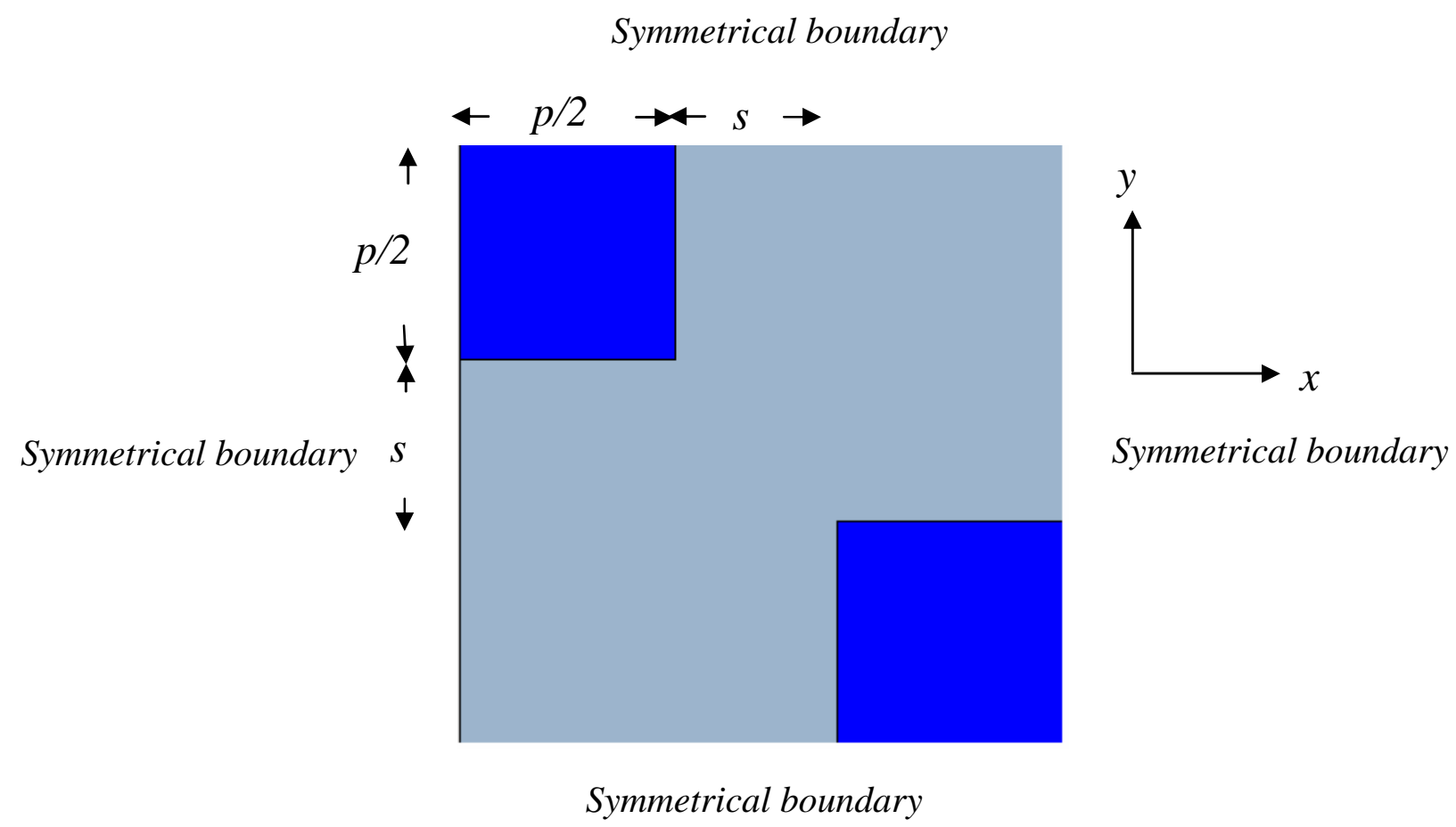

Figure 5.24 - Symmetrically reduced triangular model with symmetrical boundaries. 
These are shown as divided by two due to the symmetrical boundary conditions applied to the model. The porosity of a sample $\phi$ is defined as

$$
\phi=\frac{\text { pore_area }}{\text { total_area }}
$$

Using the parameters from the reduced models, the porosity of the rectangular and triangular models are given by equations (5.4) and.(5.5) respectively.

$$
\begin{aligned}
& \phi=\frac{p^{2}}{(p+s)^{2}} \\
& \phi=\frac{p^{2}}{2(p+s)^{2}}
\end{aligned}
$$

Energy reflection coefficients were measured using the same method as used in section 5.6. Using these measurements, and the known solid aluminium acoustic impedance, equation (5.2) was used to find the unknown porous layer impedances. Wave velocities were calculated using the same method as used in section 5.2. The excitations used were plane wave 50 and $150 \mathrm{MHz}$ Blackman pulses applied to all elements and excited along the $z$-axis. The finite element size used was $25 \mathrm{~nm}$ to provide adequate spatial resolution for the models with the smallest aluminium wall thickness. Simulation time was $40 \mathrm{~ns}$, providing enough time for a wave to reach the porous layer/solid interface and reflect back. Energy integrals were calculated by defining 2D planes in $x$ and $y$ located at desired points along the $z$-axis. Performing these measurements, the variation in the rectangular and triangular model velocity, reflectivity, and acoustic impedance with sample porosity and frequency were found.

\subsubsection{Velocity}

Figure 5.25 shows the variation in the rectangular and triangular geometry velocities with sample porosity at 50 and $150 \mathrm{MHz}$. Given the different sample architectures, sample velocity was plotted against porosity to allow direct comparison between samples of differing 
architectures. Observe that the $50 \mathrm{MHz}$ and $150 \mathrm{MHz}$ rectangular model velocity traces overlap, as do the $50 \mathrm{MHz}$ and $150 \mathrm{MHz}$ triangular model traces, showing the sample velocity is independent of the wave frequency. This is due to the small substructure dimensions of the material relative to the wavelength. The frequency was increased further to $200 \mathrm{MHz}$ for specific porosities and still no change in velocity was observed.

The velocity though is observed to show dependence on the sample architecture and sample porosity. In the limiting case of $0 \%$ porosity (solid aluminium), both the triangular and rectangular model velocities tend to the aluminium $\mathrm{P}$ wave speed of $6300 \mathrm{~ms}^{-1}$. For values of porosity up to $20 \%$ the velocities for both architectures are the same, decreasing from the $\mathrm{P}$ wave speed as porosity is increased. As porosity is increased further, at approximately $25 \%$ the dependence on architecture is first observed as the traces begin to separate. By $40 \%$ the difference is significant where the velocity for the triangular model is approximately $5270 \mathrm{~ms}^{-1}$ and for the rectangular model just under $5400 \mathrm{~ms}^{-1}$. This separation occurs since as the porosity is increased, the guided wave propagation in the two types of architectures is different. For the rectangular model, propagation remains as a plate wave regardless of porosity due to the structure of the aluminium. For the triangular model, as the porosity is reduced the architecture causes the formation of bar like zones with very little coupling between adjacent bars, and becomes dominated by the bar mode. In the limit as porosity is increased towards $100 \%$, the rectangular model trace asymptotically approaches the $S_{0}$ Lamb mode velocity of $5400 \mathrm{~ms}^{-1}$ given by equation (2.5). The triangular model asymptotically approaches the fundamental bar mode velocity of $5090 \mathrm{~ms}^{-1}$ given by equation (2.10) using a Young's modulus of $70 \mathrm{GPa}$ for aluminium, calculated from the bulk aluminium $\mathrm{P}$ and $\mathrm{S}$ wave speeds.

The above velocities were measured with the pores assumed void of any fluid. Since the PAA samples were immersed in water during the experimental work, the velocity measurements were repeated for the models at 50 and $150 \mathrm{MHz}$ with water filled pores to see if any changes in the velocities occurred. As with the $2 \mathrm{D}$ modelling, there were no significant differences between the water filled and void pore measurements. The water filled measurements were repeated for select porosities up to $200 \mathrm{MHz}$ where again no significant changes were observed. 


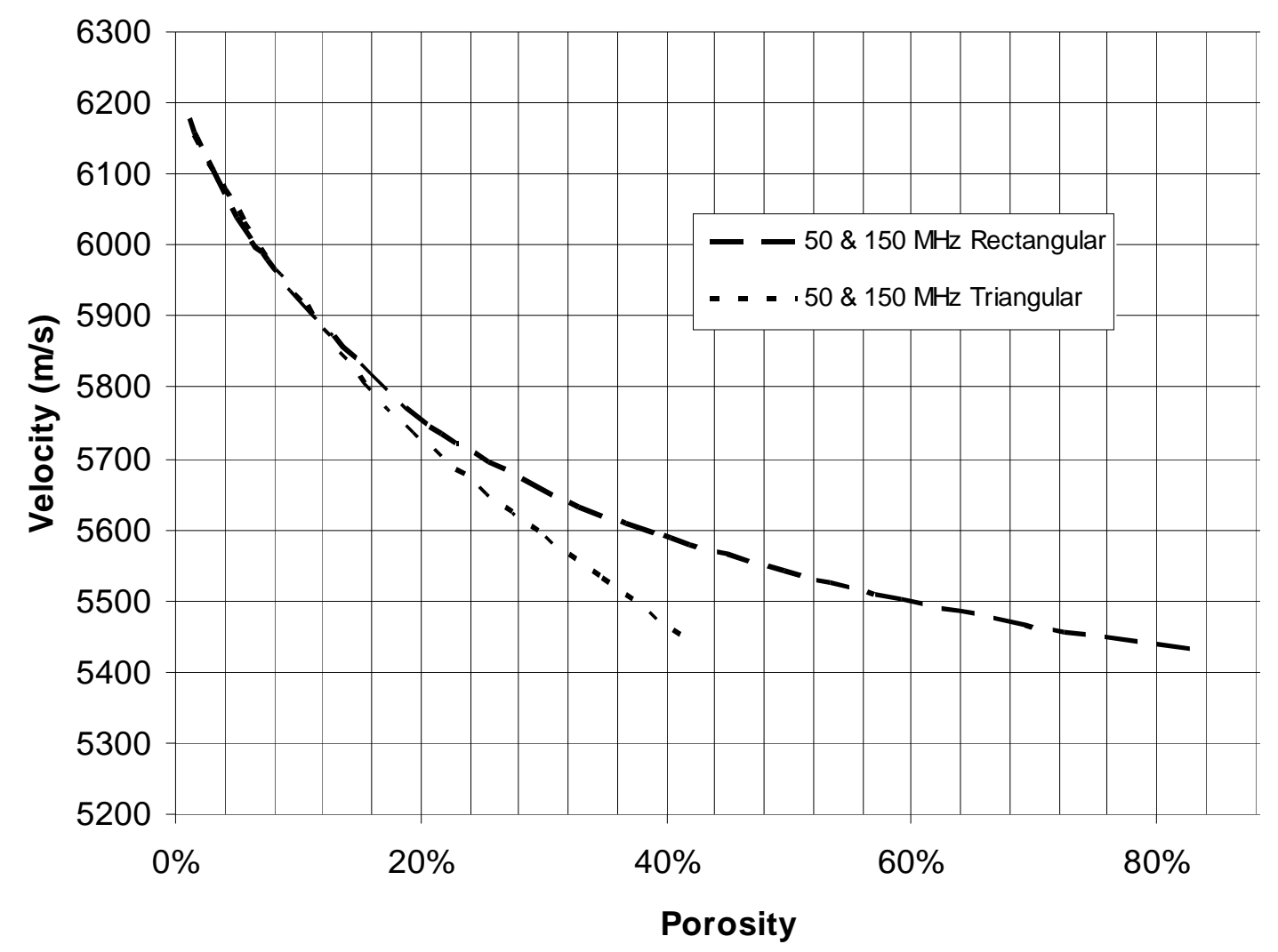

Figure 5.25 - Rectangular and triangular model velocities versus porosity for 50 and $150 \mathrm{MHz}$ waves.

\subsubsection{Reflectivity}

The variation in the energy reflection coefficient (percentage energy reflected) at the porous/solid aluminium interface with porosity, frequency, and architecture is shown in Figure 5.26. As can be observed there is no distinction between any of the four graphs, meaning the energy reflection coefficient is independent of both frequency and architecture, but still highly dependent upon porosity. In the limiting case of $0 \%$ porosity (solid aluminium) the reflected energy is $0 \%$ resulting in $100 \%$ transmission. For low porosities $10 \%$ or less, the percentage energy reflected is very low implying the propagating waves are not significantly guided for these porosities. This is due to the bulk material still being largely aluminium with a small total pore area. At the triangular model maximum porosity of $45 \%$, the percentage reflected energy is around $12 \%$ suggesting a significant impedance mismatch. By increasing the porosity to $60 \%$, the reflected energy almost doubles to approximately $23 \%$. 


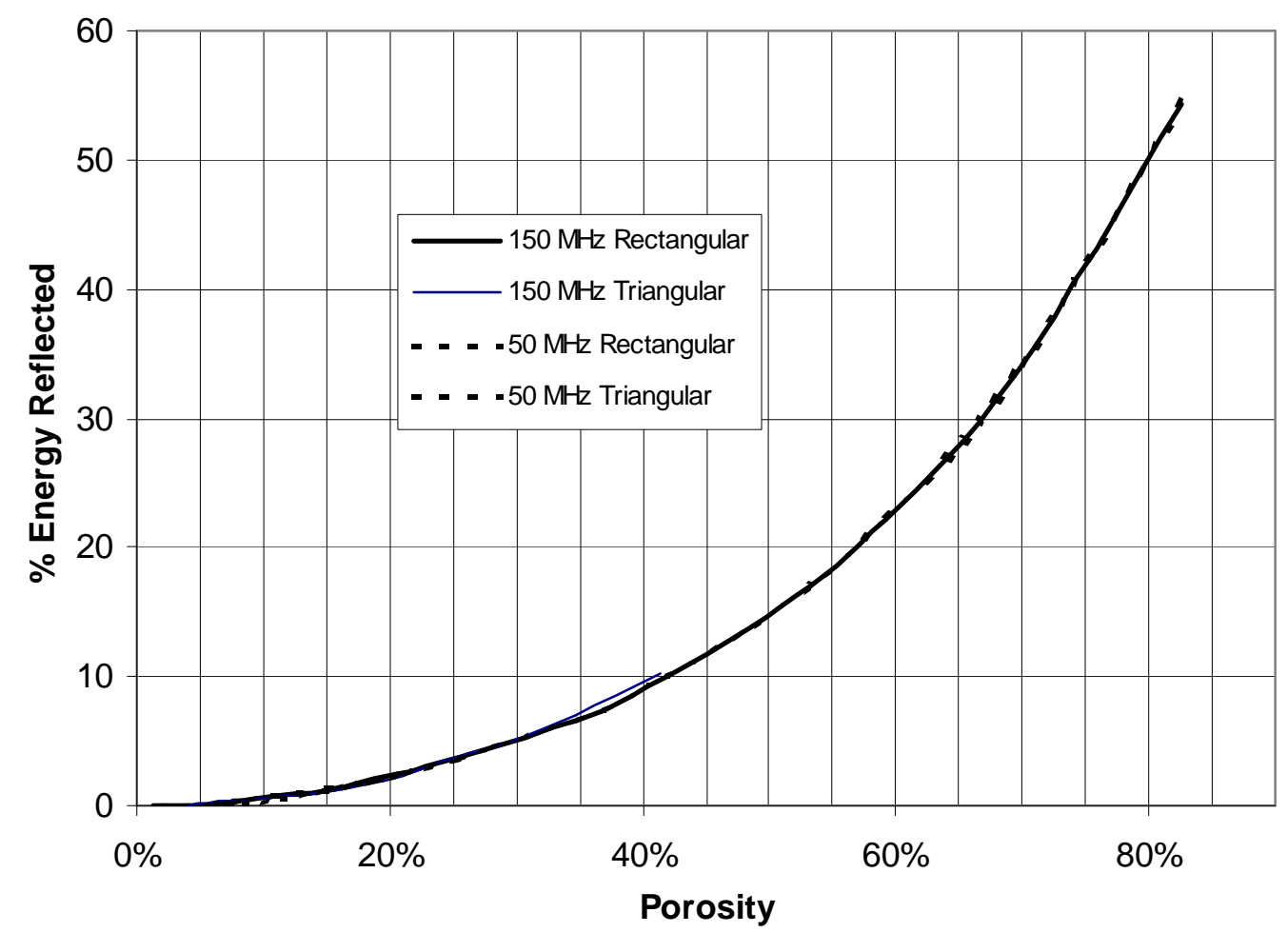

Figure 5.26 - Rectangular and triangular model energy reflection coefficients versus porosity at 50 and $150 \mathrm{MHz}$.

Extending beyond this to a very high porosity of $80 \%$, the energy reflection coefficient reaches $50 \%$, however physical samples with such porosity are not realistic. Figure 5.26 shows the variation in the reflectivity of the samples is not linear, but appears to vary with the square of the sample porosity. This is expected as by changing the sample porosity the impedance $\mathrm{Z}_{1}$ changes, which by the nature of equation (2.13) means the pressure reflection coefficient is varying non-linearly. To calculate the energy reflection coefficient equation (2.13) is squared as according to equation (2.16), resulting in the observed quadratic behaviour.

\subsubsection{Acoustic Impedance}

Using the energy reflection coefficients from the previous section, and the known solid aluminium acoustic impedance, the variations in the rectangular and triangular model acoustic impedances could be found. Since the reflection coefficients were found to be independent of both frequency and architecture, according to equation (5.2) so would the acoustic impedances. Therefore Figure 5.27 only plots the variations in the rectangular model acoustic impedance with porosity at $150 \mathrm{MHz}$. 


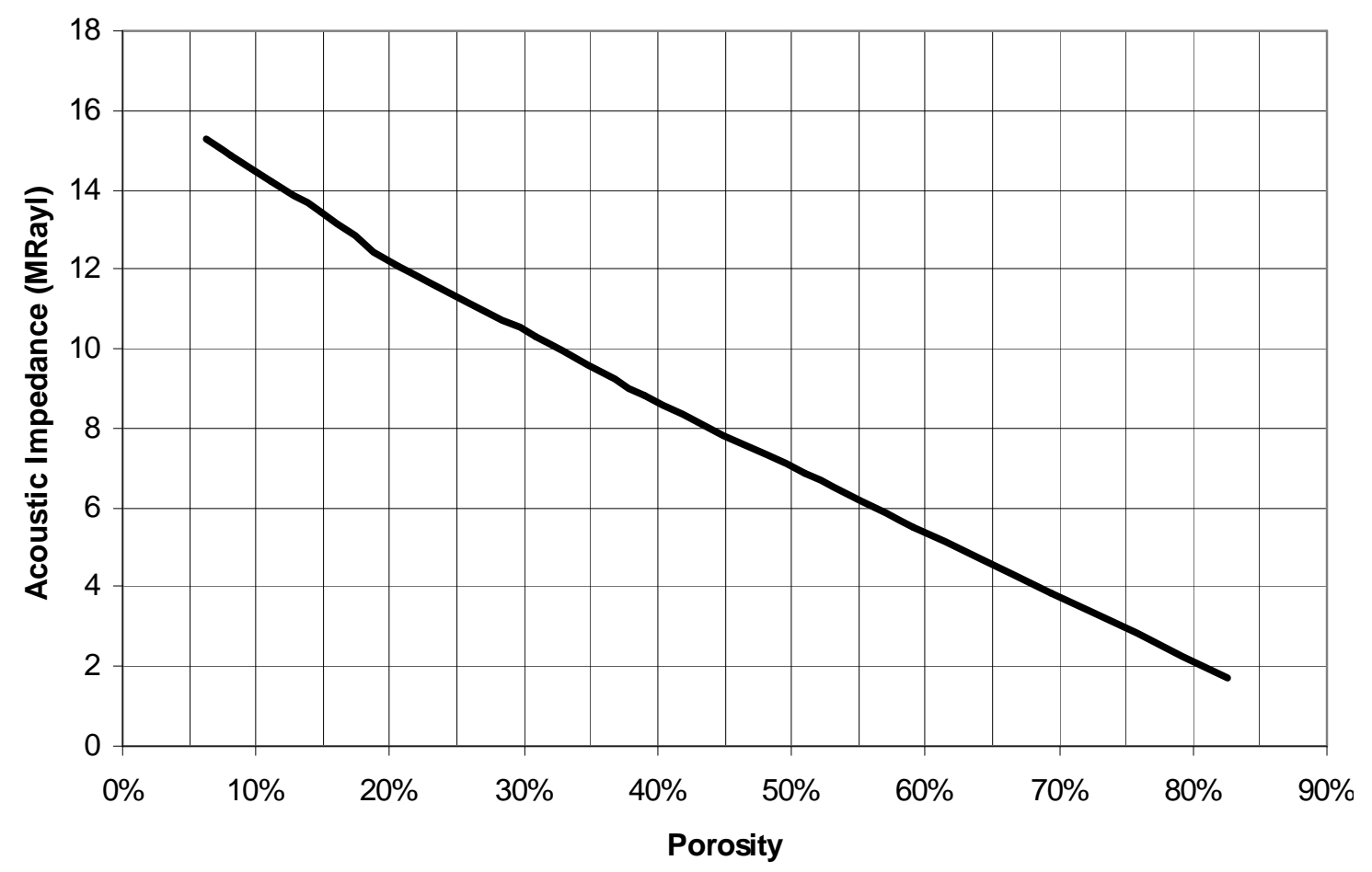

Figure 5.27 - Rectangular model acoustic impedance versus porosity at $150 \mathrm{MHz}$.

A highly linear relationship between sample acoustic impedance and porosity is observed, with a wide range of acoustic impedances that can be generated by simply varying the sample porosity. Increasing the porosity from 0 to just $10 \%$, the acoustic impedance of the layer is reduced from 17 MRayl (solid aluminium) to less than 15 MRayl. In the range of 40-50\% porosity, comparable to the porosity range found for the type 2 and type 3 samples, impedances in the range 7-9 MRayls can be realised. Finally, at $60 \%$ porosity the impedance is reduced almost to 5 MRayl. Given the type 3 sample porosities vary between 45-50\% porosity and are still very robust, the fabrication of a $60 \%$ porosity sample is very realistic. This would allow given the type 1 samples possess porosities of 20-25\% - an impedance range of 5-13 MRayl for these samples. This is an ideal impedance range for transducer matching purposes.

Acoustic impedance measurements were not repeated with water filled pores as it was found fluid loading had little influence on the measured velocity. As described in section 2.2 velocity and density determine the characteristic acoustic impedance of a medium. It was also shown in section 5.4 that the amount of energy propagating in the water relative to the aluminium is insignificant, specifically for the dimensions concerned with here. This means the consideration of just the fast wave properties by setting the pores to be void is a fair approximation to determine the wave-guiding properties of these porous samples. 


\subsection{Significance of the Modelling Results - PAA Transducer Matching Layers}

The results from the 2D and 3D FEM revealed the wave guiding properties of a collinear microstructured material display compared to a bulk solid. The combination of these properties would be ideal for a high frequency transducer matching material, showing significant advantages over the currently used materials. Matching layers, as described in section 2.3.3, are intermediate layers placed between two poorly acoustically matched media to increase the energy coupling between them.

In medical ultrasound, the high acoustic impedance (>30 MRayl) of commonly used piezoceramics for transducer active elements [96], [97], and the low acoustic impedance $(<1.6$ MRayl) of biological tissues [40], is a large acoustic impedance mismatch, resulting in poor energy transmission into the tissue samples. Calculating the energy transmission coefficient at the interface of these two materials using equations (2.16) and (2.18) results in a transmission coefficient of just 0.19 ; this acoustic mismatch can be reduced significantly by including one or more acoustic matching layers into the transducer. As reported in section 2.3.3, matching materials with acoustic impedances in the range of 3 to 14 MRayl are often required unfortunately there are no useful single phase solids that fall in this range.

To realise these impedances, the state of the art technique is to use an epoxy matrix loaded with a fine powder, where the fractional loading of the powder determines the overall impedance and attenuation of the layer. In [98], alumina powder with a $3 \mu \mathrm{m}$ particle size was mixed with epoxy for matching layers operating in the 20-70 MHz range. In [99] and [100] silver powder of a similar particle size was used. The problem with these loaded materials is the presence of the particles causes them to be intrinsically lossy as the sub-wavelength scatterers cause diffusive scattering of the ultrasound (see section 2.5.1.2). This scattering shows significant frequency dependence and hence loaded epoxies are often used to make lossy backing materials to dampen the active element of a transducer [98].

For very high frequencies e.g. $100 \mathrm{MHz}$, these layers must be made less than $8 \mu \mathrm{m}$ thick in order to satisfy the quarter wavelength requirement. This means even finer submicron sized loading powders must be used to ensure a uniform matching layer is obtained. This was 
demonstrated in [101] and [102] where high quality alumina powder with $30 \mathrm{~nm}$ average particle size was mixed with epoxy realising impedances in the range of 2.8 to 5.1 MRayl. This reduced the attenuation in the matching layer considerably at high frequencies compared to those made with $3 \mu \mathrm{m}$ particles [98]. Attenuation was $15 \mathrm{dBmm}^{-1}$ at $40 \mathrm{MHz}$ and significantly increased with the fourth power of frequency beyond this. This was recently improved in [103] where cerium oxide with $20 \mathrm{~nm}$ average particle size was used, increasing the upper limit of adjustable impedance to $7 \mathrm{MRayl}$, with a quoted value for attenuation in the layer of 500 $\mathrm{dBmm}^{-1}$

$100 \mathrm{MHz}$. These matching layers are very lossy at frequencies where signal strength is critical, and require the use of very fine loading powders.

The proposed solution to these lossy, scattering, matching layers, is to use porous nanostructured materials with a regular highly collinear structure to act as a waveguide to the incident ultrasound waves. The waveguiding properties mean there would be very little scattering in these layers, with the only attenuation due to the material absorption. Less regular porous filtration membranes have been shown in [104] and [105] to improve energy coupling significantly in air-coupled ultrasound applications over the frequency range of 0.3-3 MHz. The modelling performed on highly collinear microstructured materials demonstrated properties such as the normalisation of incident ultrasonic waves, a wide range of adjustable acoustic impedances, and no dispersion. The independence of the sample velocity on frequency is an important characteristic not found in current matching layers. The scattering characteristic of epoxy/powder layers causes the distortion of generated pulses, modifying the spectrum of the wave. 


\section{Chapter 6}

\section{Experimental Characterisation of PAA Samples}

With the FEM performed, verification of the waveguiding phenomena observed in the modelling was obtained by performing experimental measurements on the PAA samples. As mentioned in section 4.1, the PAA samples were not porous aluminium, but did possess similar acoustic properties. This meant the exact values of these properties will differ somewhat between experiment and modelling, but the dependence of these upon frequency, architecture, porosity etc. should be the same. Experimentally measured PAA velocity, acoustic impedance, and attenuation measurements are presented in this chapter. The velocity and acoustic impedance measurements are compared with the modelling results from the previous section, and the attenuation measurements compared with values for the state of the art matching layers found in the literature.

\subsection{Velocity}

The variations in the PAA sample velocity measurements with angle of incidence, sample structure, and frequency are presented in this section. These results are compared with those generated from the modelling reported in section 5.7.2. These measurements were made using the three different frequency transducers driven with $50 \mathrm{MHz}, 100 \mathrm{MHz}$, and $175 \mathrm{MHz}$ pulses 
respectively. These were single cycle sine pulses centred at the above transducer frequencies with a $20 \%$ Blackman window [112] applied to each. Windowing prevented sharp discontinuities in the time domain and resulted in a smooth frequency spectrum with significantly reduced lobes. The transmission measurement experimental configuration described in section 3.1.1 was used to perform the measurements.

Measurements were performed on the three different in-house fabricated PAA samples, a commercial PAA sample, and a high purity solid aluminium sample. The angle of incidence of the transmitting transducer was varied from $0^{\circ}$ to $40^{\circ}$ degrees and the group velocities measured. Measuring the velocity over these angles provided an indication of the normalising capabilities of each of the samples under test. Equation (3.3) was used to calculate the group velocities. Since the samples were rigid, the sample thicknesses could be measured using a Mitutoyo dial micrometer. The measuring needle restoring spring was removed from the micrometer to ensure minimal compressional force was applied to the sample during measurement. The thickness measurements were accurate to within $\pm 5 \%$.

\subsubsection{Solid Aluminium}

The measurements for a solid aluminium sample are shown in Figure 6.1 where the variations in the group velocity are plotted with transducer angle of incidence from $0^{\circ}$ to $40^{\circ}$ at $50 \mathrm{MHz}$. For normal incidence only the bulk aluminium $P$ wave with a velocity of $6300 \mathrm{~ms}^{-1}$ was observed as expected. As the angle was increased to small angles $\left(<10^{\circ}\right)$ beyond normal both the $\mathrm{P}$ wave and an $\mathrm{S}$ wave with a velocity of $3100 \mathrm{~ms}^{-1}$ were measured. After $14^{\circ}$ the $\mathrm{P}$ wave was no longer observed, only the $\mathrm{S}$ wave. This was the case until $28^{\circ}$ where for angles greater than this the $\mathrm{S}$ wave was also no longer observed. This is in agreement with the theory from section 2.3.2 where it was shown for a water/aluminium interface the theoretical $\mathrm{P}$ wave critical angle was found to be $14^{\circ}$, and the $\mathrm{S}$ wave critical angle $29^{\circ}$. For any angles of incidence greater than these the respective waves travel along the surface of the interface, thus going undetected in a transmission measurement. 


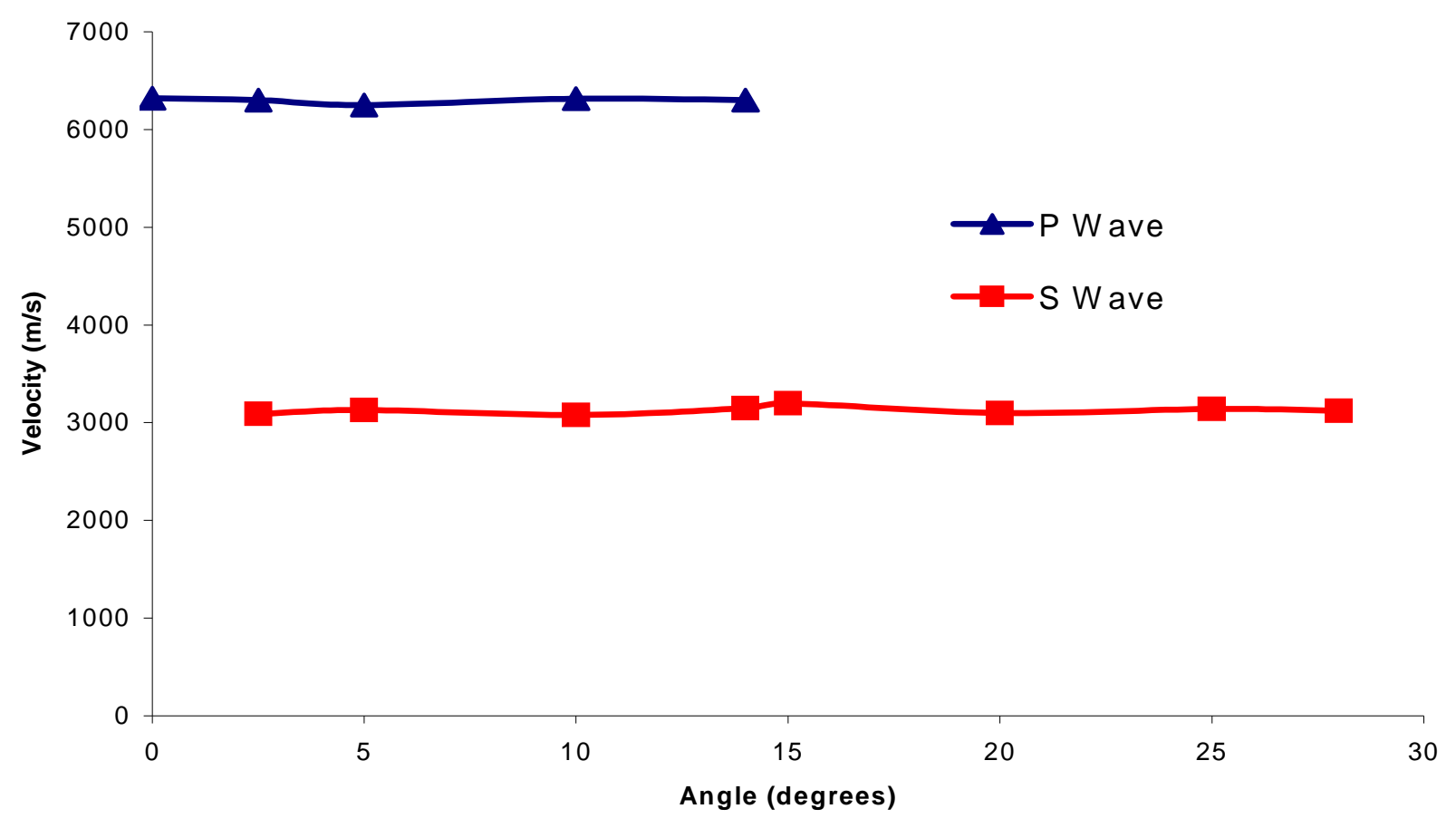

Figure 6.1 - Variations in group velocity with transducer angle of incidence at $50 \mathrm{MHz}$ for solid high purity aluminium sample.

The energy content of the measured $\mathrm{P}$ and $\mathrm{S}$ waves with angle of incidence were consistent with those plotted in Figure 2.12. This shows the expected fraction of energy transmitted as a $\mathrm{P}$ and an $\mathrm{S}$ wave and the expected fraction of the reflected energy with angle of incidence at a solid/aluminium interface. In the range of $10-14^{\circ}$ angle of incidence experimental measurements found significant energy in both the $\mathrm{P}$ and $\mathrm{S}$ wave solid aluminium components with the S wave energy approximately $25-40 \%$ of the $\mathrm{P}$ wave energy over this range.

\subsubsection{In-house Fabricated PAA Samples}

The variations in the group velocity for the in-house fabricated PAA samples with angle of incidence at $50 \mathrm{MHz}$ are shown in Figure 6.2. For all three samples only a single wavefront of constant velocity was received up to angles of $40^{\circ}$. The variations in the measured velocities for all three samples were in good agreement with the results produced from the modelling, which predicted a dependence of wave velocity on sample porosity.

For type 1 samples the porosity was measured to vary between $23-28 \%$. From the triangular model (closely resembling the type 1 sample architecture) simulation results from section 5.7, 
velocities were calculated in the range of $5600-5700 \mathrm{~ms}^{-1}$ over this porosity range. This is in good agreement with the experimentally measured velocities for the type 1 samples in Figure 6.2 that varied between 5816-5890 $\mathrm{ms}^{-1}$. For the type 2 and type 3 samples, porosities were measured to vary between $40-44 \%$ and $46-50 \%$ respectively. From the rectangular model simulation results, the velocity did not vary significantly over the range of 40-50\% porosity with simulated velocities in the range $5550-5600 \mathrm{~ms}^{-1}$. From Figure 6.2 the measured type 2 sample velocities varied between $5662-5730 \mathrm{~ms}^{-1}$, and for the type 3 samples velocity measurements were on average less than this, varying in the range $5620-5685 \mathrm{~ms}^{-1}$. Both of these ranges are in good agreement with the rectangular model results.

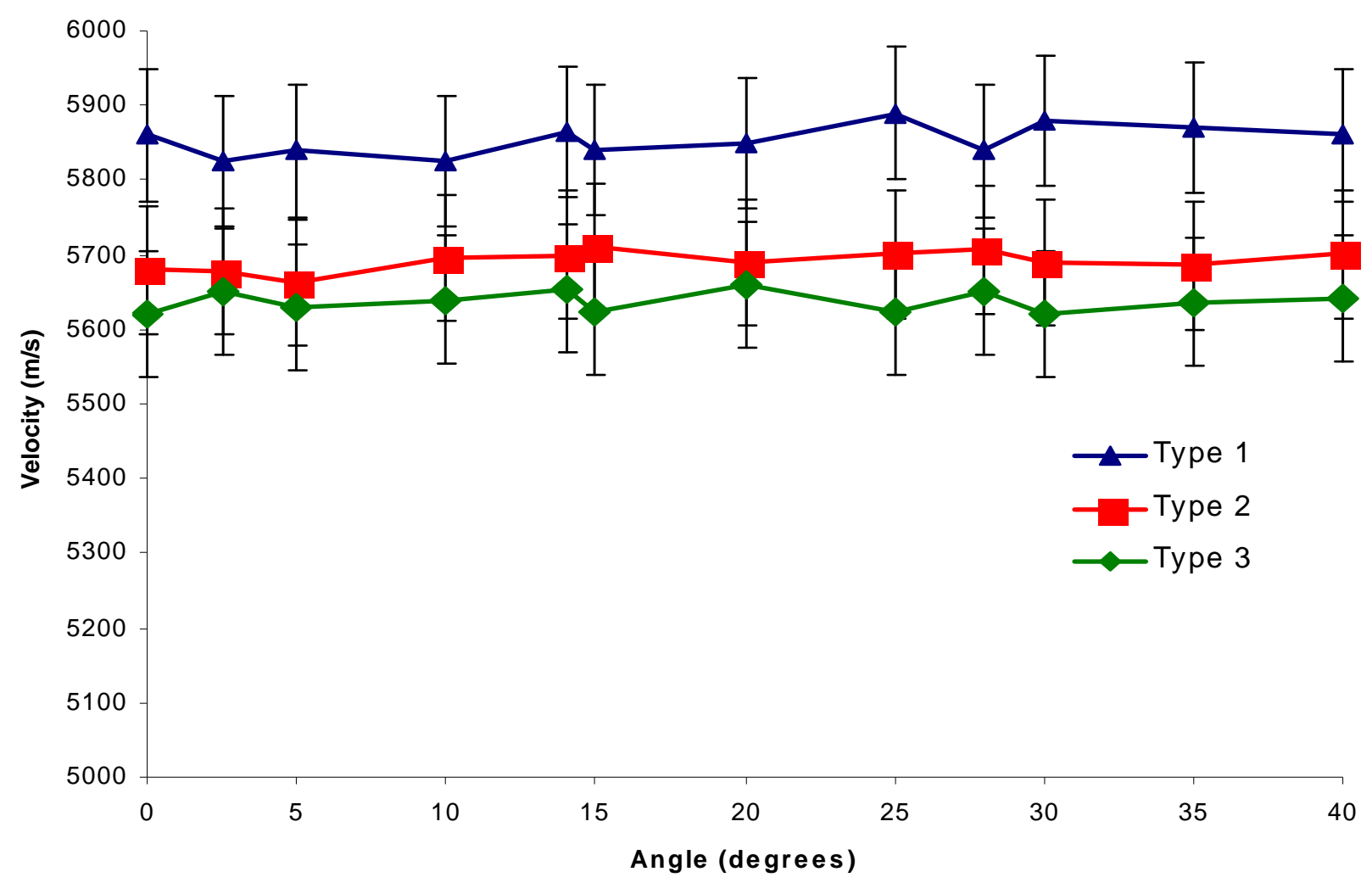

Figure 6.2 - Variations in sample group velocity with transducer angle of incidence at $50 \mathrm{MHz}$ for the three in-house fabricated PAA samples. 


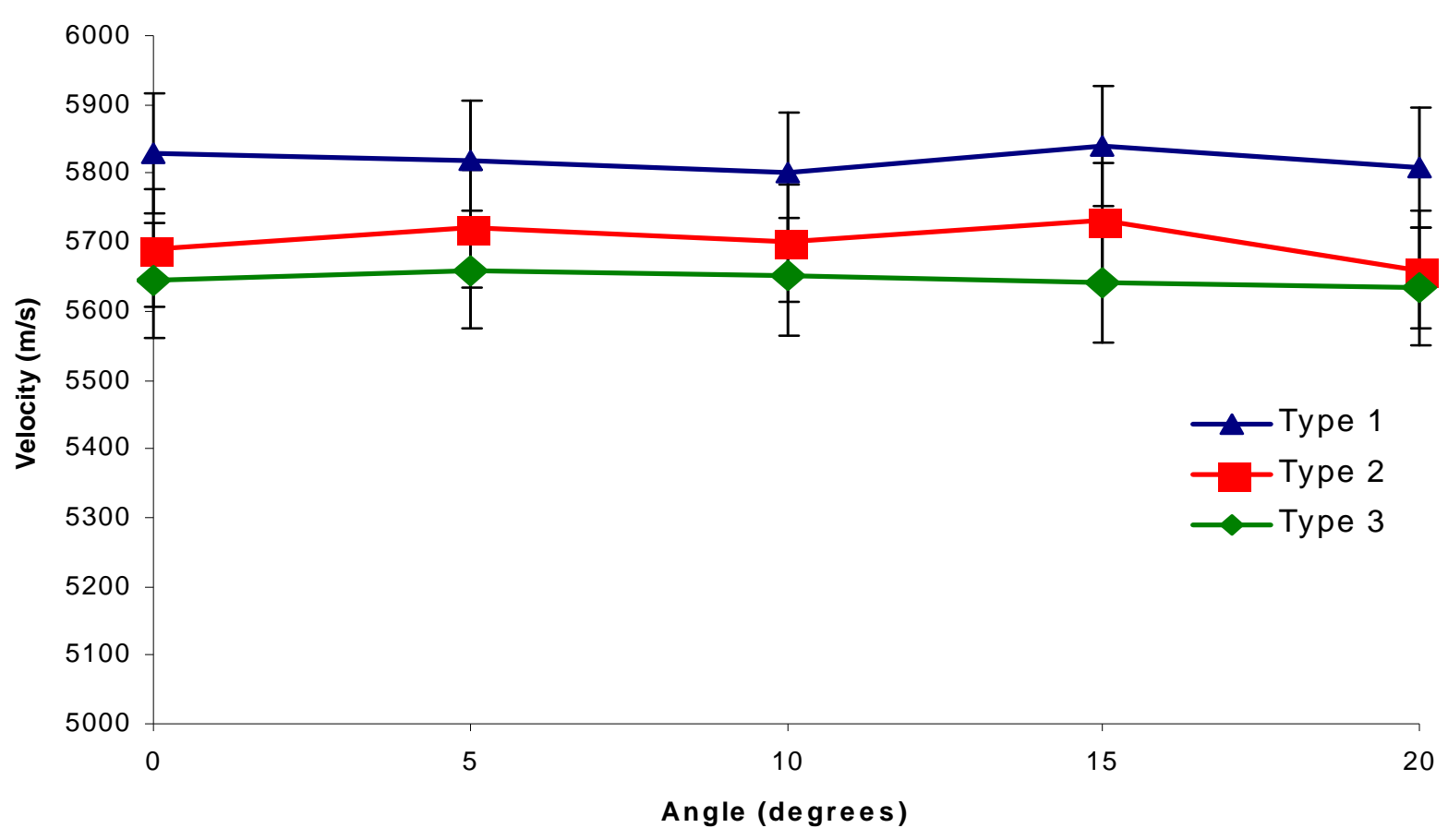

Figure 6.3 - Variations in sample group velocity with transducer angle of incidence at $100 \mathrm{MHz}$ for the three in-house fabricated PAA samples.

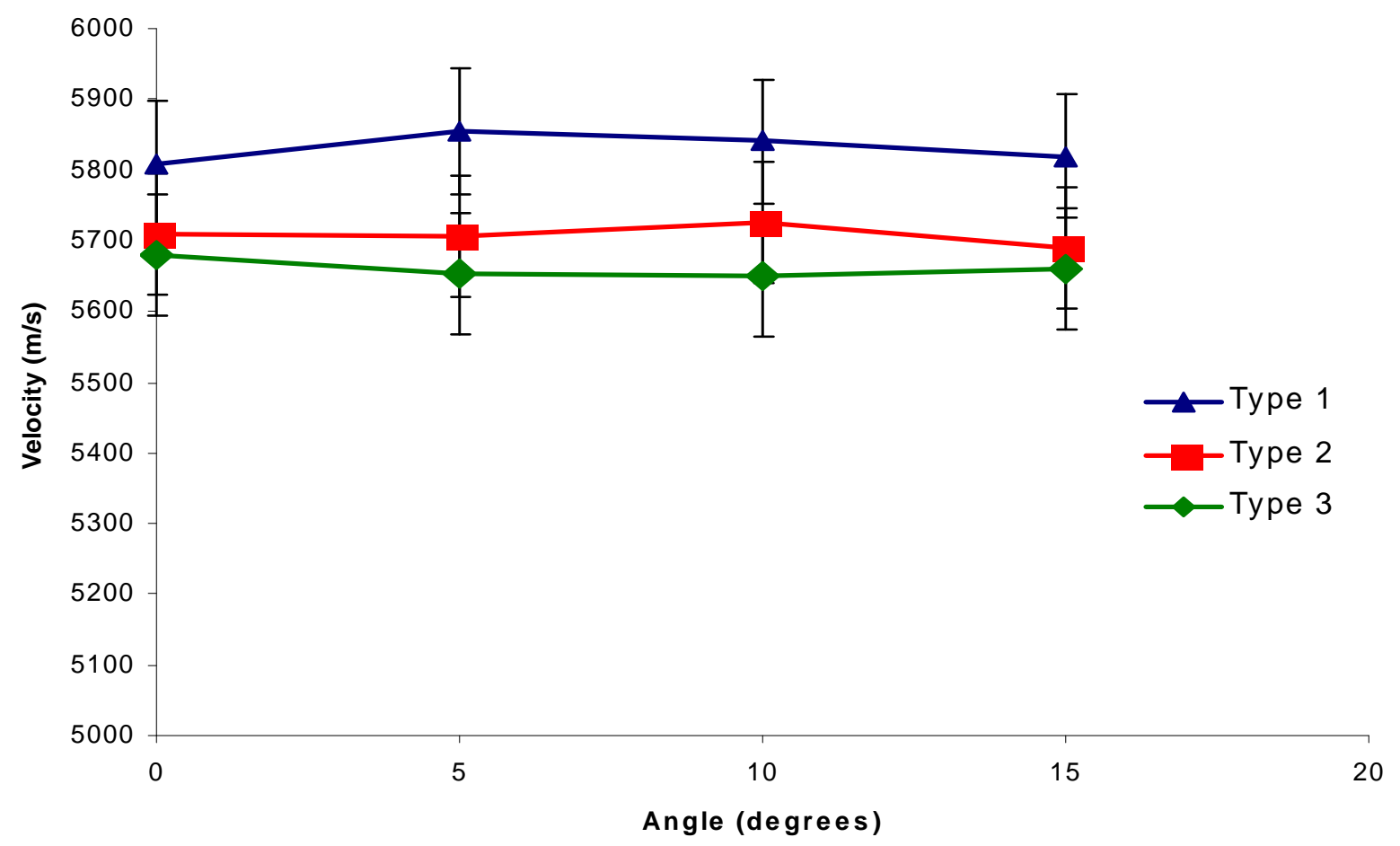

Figure 6.4 - Variations in sample group velocity with transducer angle of incidence at $175 \mathrm{MHz}$ for the three in-house fabricated PAA samples. 
The experimentally measured velocities were all larger than those obtained from the modelling, but within approximately $5 \%$. This is expected since the bulk acoustic properties of amorphous alumina are larger than those of solid aluminium. The velocity measurements were then repeated at $100 \mathrm{MHz}$ and $175 \mathrm{MHz}$, which are plotted in Figure 6.3 and Figure 6.4 respectively. At $100 \mathrm{MHz}$ signals were significantly attenuated and as a result measurements were made to an angle of incidence of only $20^{\circ}$. This was reduced further to $15^{\circ}$ at $175 \mathrm{MHz}$. Once again the velocity for the three samples can be observed to be constant for all angles of incidence. The main point to note is the increase in frequency has produced no significant variation in the velocities, with the same velocity difference observed between all three samples at the three different frequencies.

Figure 6.5, Figure 6.6, and Figure 6.7 plot the velocity variations with angle of incidence at all three frequencies for each of the type 1, type 2, and type 3 samples respectively. This allows direct comparison between velocity and frequency for the same sample types. From these it is confirmed the variation in frequency from $50-175 \mathrm{MHz}$ has no significant influence on the measured velocity for any of the samples.

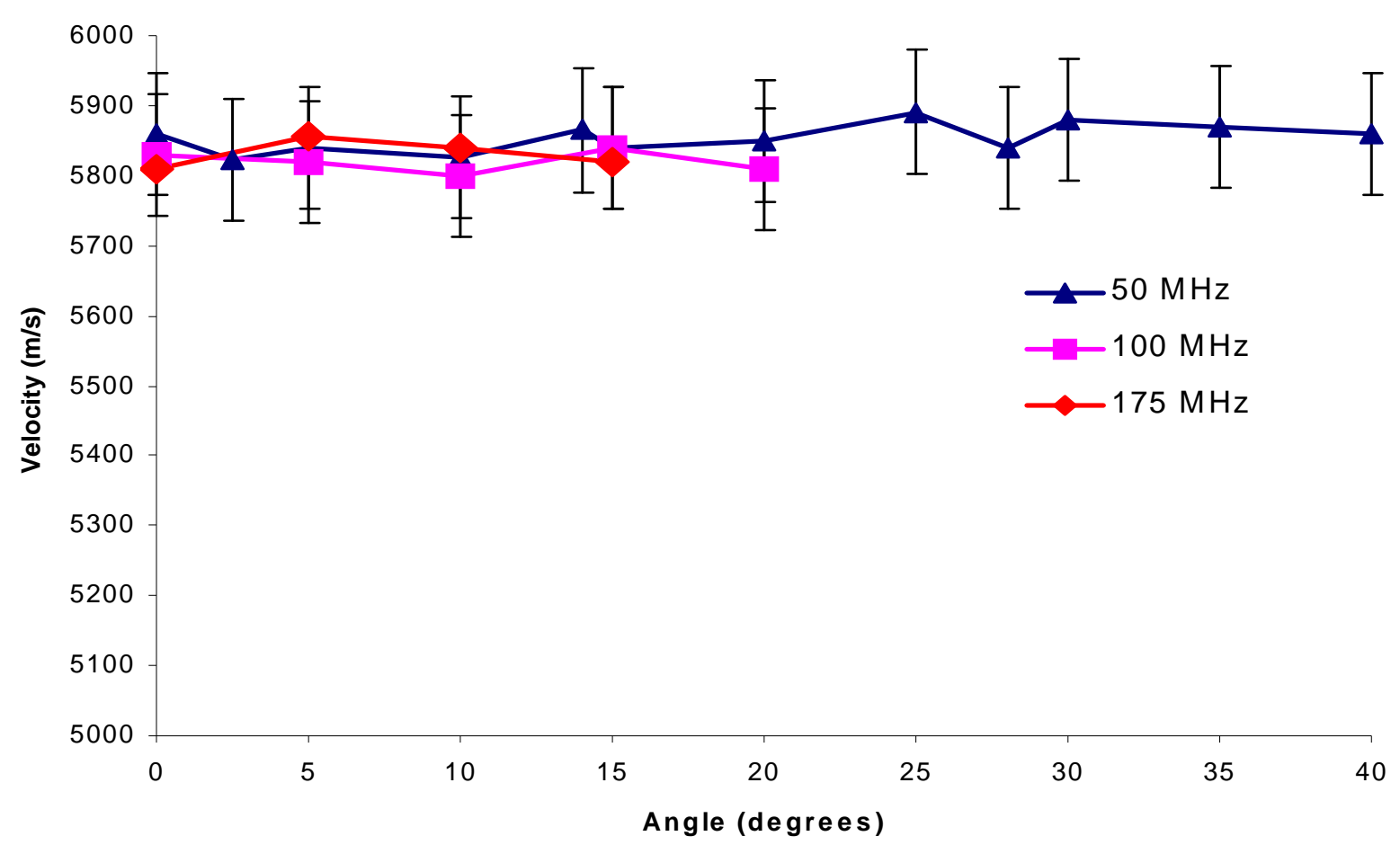

Figure 6.5 - Velocity versus angle of incidence at $50 \mathrm{MHz}, 100 \mathrm{MHz}$, and $175 \mathrm{MHz}$ for the type 1 samples. 


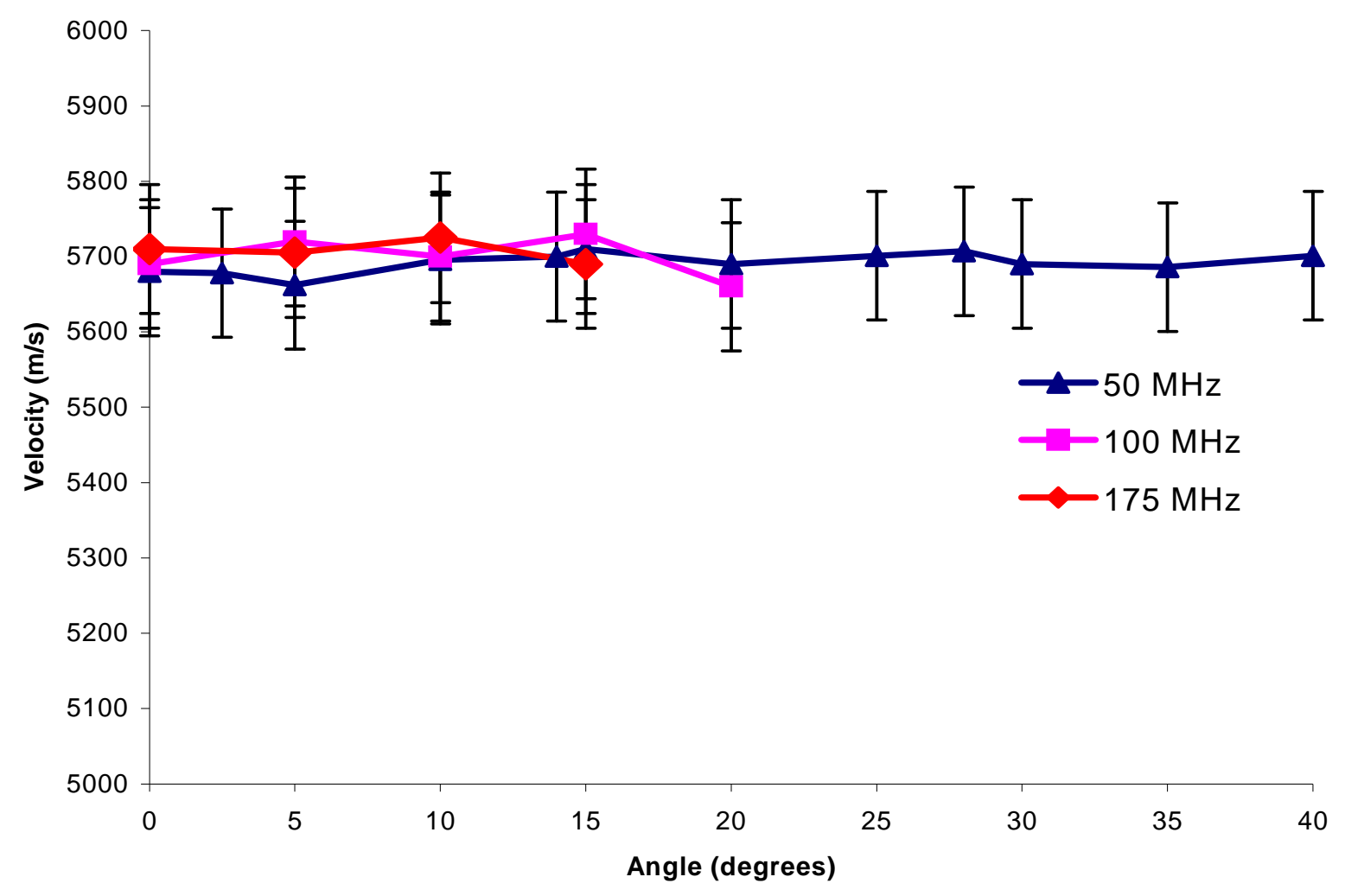

Figure 6.6 - Velocity versus angle of incidence at $50 \mathrm{MHz}, 100 \mathrm{MHz}$, and $175 \mathrm{MHz}$ for the type 2 samples.

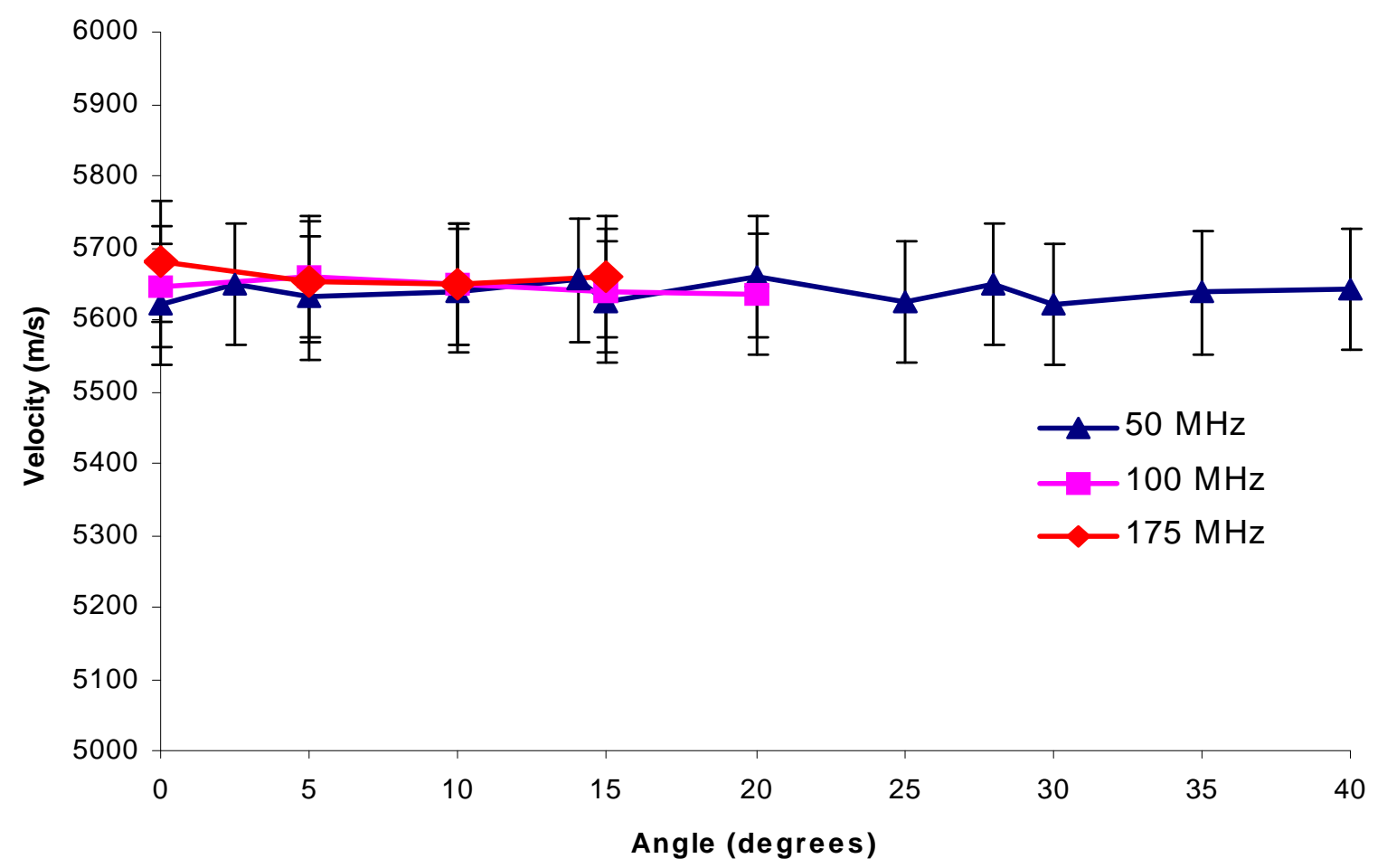

Figure 6.7 - Velocity versus angle of incidence at $50 \mathrm{MHz}, 100 \mathrm{MHz}$, and $175 \mathrm{MHz}$ for the type 3 samples. 
These measured velocity results at the three frequencies are therefore consistent with those observed in the modelling. This is because the experimentally measured velocities were found to be independent of the propagating frequency, and appeared to be independent of architecture, but dependent upon porosity. Velocity differences were consistently observed between the type 2 and type 3 samples for all three frequencies yet both types display similar architectures. Type 1 and type 2 samples contain very similar dimensions, but very different porosities, and hence show significantly different velocity measurements.

Ignoring sample type and architecture, the variations in the average measured velocities with average porosities are plotted for $50 \mathrm{MHz}, 100 \mathrm{MHz}$, and $175 \mathrm{MHz}$ in Figure 6.8. Here the $25 \%$ porosity measurements correspond to the type 1 sample velocity measurements at all three frequencies, and the next two porosities the type 2 and type 3 velocity measurements performed at all three frequencies. This is in good agreement with the velocity versus porosity curve from the modelling results in Figure 5.25.

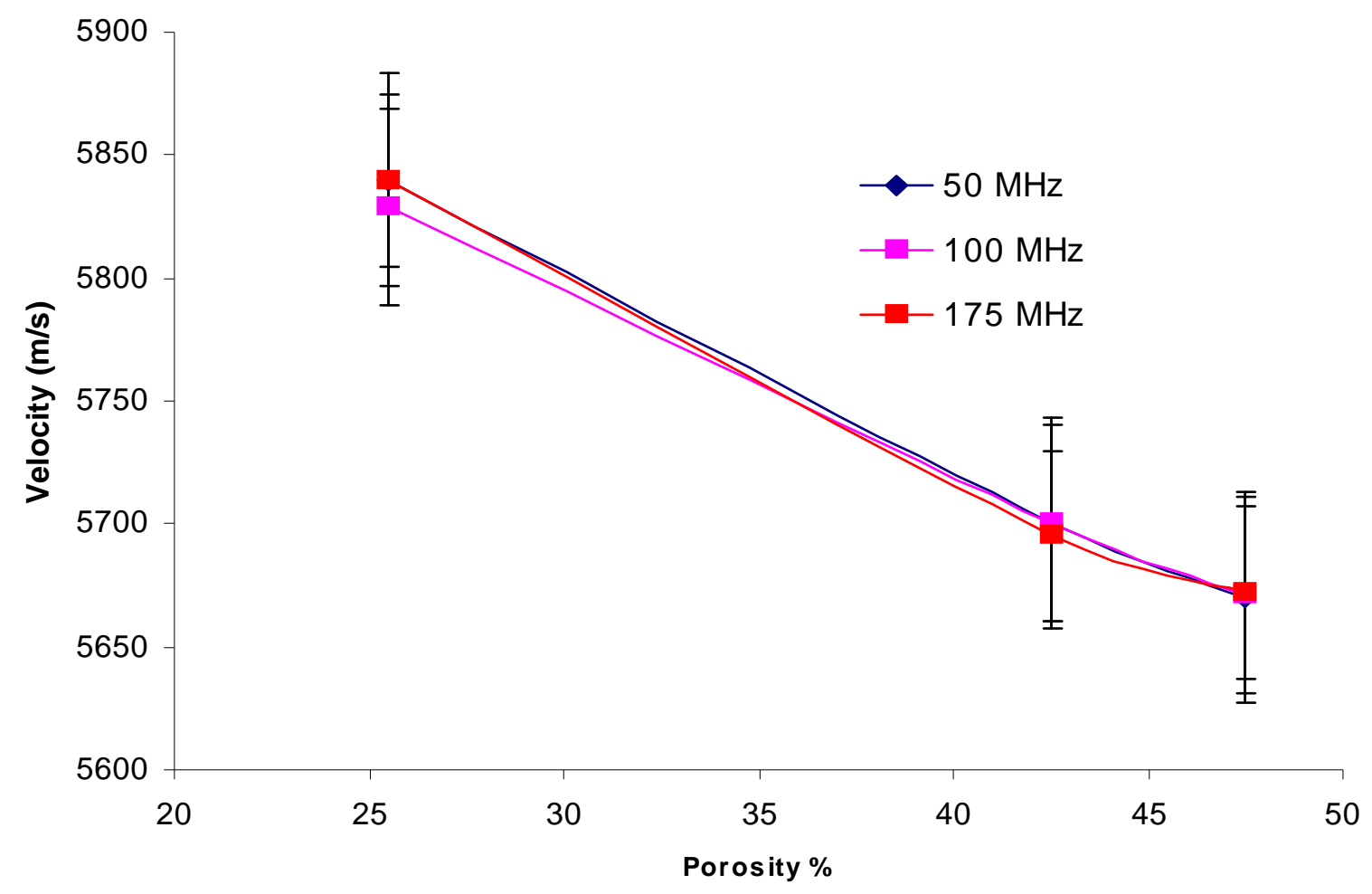

Figure 6.8 - Average sample velocity versus porosity at $50 \mathrm{MHz}, 100 \mathrm{MHz}$, and $175 \mathrm{MHz}$. 
For all PAA samples, the velocities were measured for angles up to $40^{\circ}$, well past the $\mathrm{P}$ and $\mathrm{S}$ critical angles that exist for the solid aluminium. The reason for this is the microstructure of the PAA samples impose a dimensional constraint relative to the incident wavelength, acting as waveguides and normalising the incident propagating waves. This is consistent with the modelling results presented in section 5.5. This allows the detection of signals over a wide range of angles of incidence. The significance of this property is signals incident beyond the critical angles potentially convey important information, but go undetected in systems that cannot normalise signals at such angles.

Analysis of the amplitudes of the waveforms measured from the PAA samples for all angles of incidence revealed only the fast guided wave and its reverberations. The slow waves observed in the modelling that propagate within the pores of the samples were not observed experimentally for any of the three sample types. This is consistent with the modelling results from section 5.4 where it was found the ratio of energy between the fast and slow waves was $30: 1$ at $20 \%$ porosity, $12: 1$ at $50 \%$, and was not until $90 \%$ where the energy partition reaches 1:1. Therefore due to the slow velocity and small signal amplitude the slow wave was hidden in the sample reverberation. To confirm this samples would have to be made many times thicker to ensure the slow wave was received before the fast wave reverberation in the plate.

\subsubsection{Commercial PAA Sample}

The commercial PAA samples velocity results are plotted in Figure 6.9 at $50 \mathrm{MHz}$. For the commercial samples, only a single wavefront was received, as was the case for the in-house fabricated samples. This is due to the pore wall dimensions of the sample being of the same order as the in house fabricated PAA samples, imposing a dimensional constraint. Velocities were also measured out to $40^{\circ}$, however the velocity was found to be highly variable in comparison to the in-house fabricated samples. The waveforms also appeared somewhat distorted and consistently lower in amplitude when compared with the in house samples. Given the poor collinearity of these samples (see Figure 4.7 and Figure 4.8) this variation in velocity and amplitude was expected since the velocity will depend on the wave path travelled and thus the irregular variation in sample porosity, and the misaligned pores creating sites for significant scattering. This was observed for measurements at normal incidence where the sample was moved around so that different parts of the sample were illuminated. 


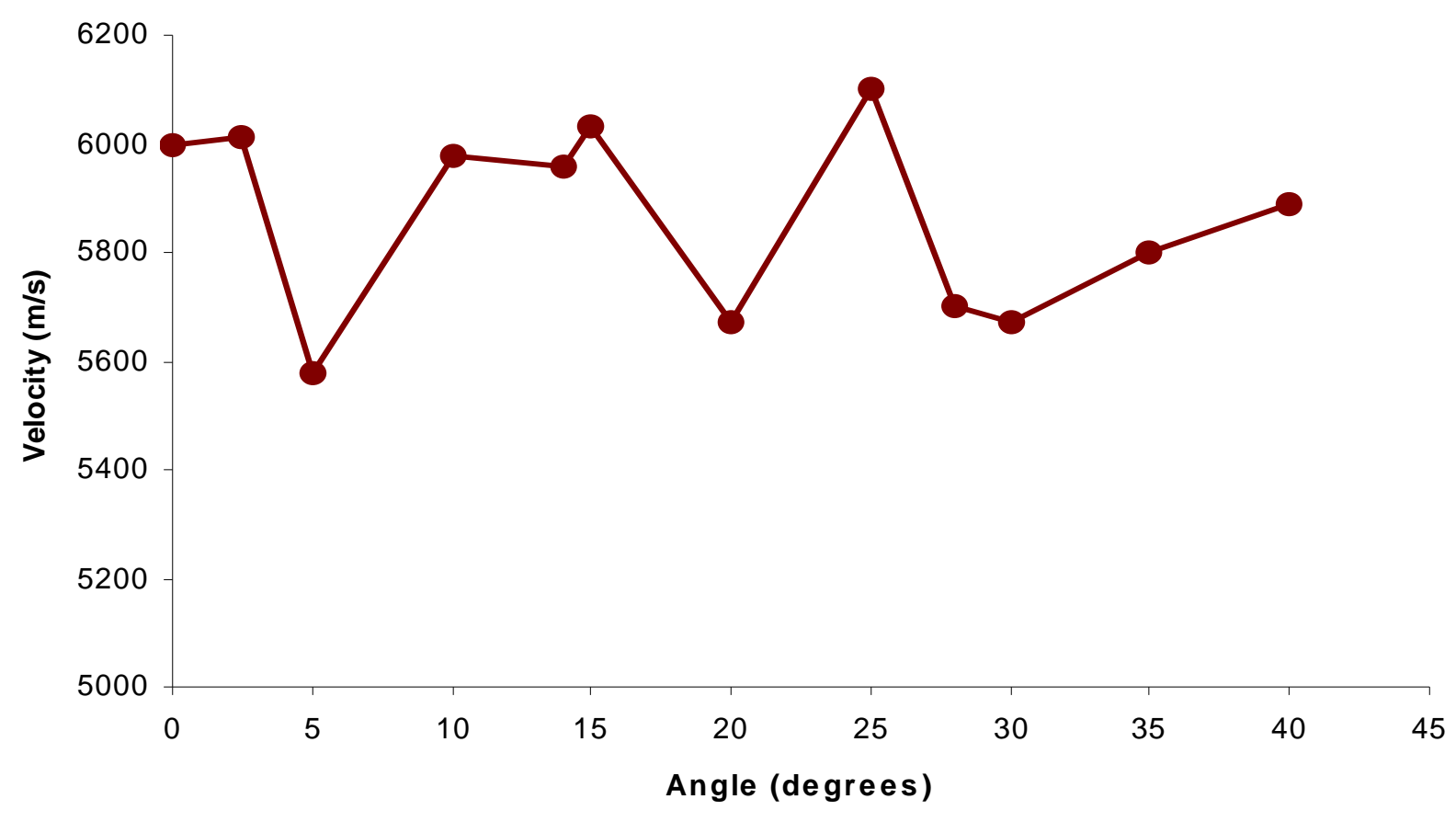

Figure 6.9 - Variations in group velocity with transducer angle of incidence at $50 \mathrm{MHz}$ for a commercial PAA sample.

Observing the oscilloscope whilst doing this, significant variations in time and amplitude of the received signals were observed. This is in contrast to that observed for the in-house fabricated PAA samples where very little temporal or amplitude variations were observed. Velocities for the commercial samples were measured in the range $5290-6195 \mathrm{~ms}^{-1}$.

\subsection{Acoustic Impedance}

The acoustic impedances of each of the in-house fabricated PAA samples were measured using the pulse-echo method described in section 3.1.2. This method involved measuring at normal incidence the amplitudes reflected from both the unknown PAA samples and a homogeneous sample of known acoustic impedance, in this case high purity aluminium. The thickness of the unknown and known samples did not need to be the same as the transducer height was adjusted until the received signal strength from a sample reached a maximum. At this height the sample was within the focal zone, located independent of the sample thickness.

Using the known acoustic impedances of the high purity aluminium and water, and the measured amplitudes from each sample, the unknown acoustic impedances of the alumina 
samples were calculated using equation (3.8). The resulting acoustic impedance measurements are summarised in Table 6.1, Table 6.2, and Table 6.3 corresponding to measurements made at $50 \mathrm{MHz}, 100 \mathrm{MHz}$, and $175 \mathrm{MHz}$ respectively. These are compared with those calculated from the modelling. The measured impedances of each type of sample were averaged over at least five samples and performed at the three different frequencies using the same waveforms as used for the velocity measurement. For all three samples the impedance measurements showed no frequency dependence. The uncertainty range in the measured impedances took into account the uncertainty in the known aluminium and water acoustic impedances.

Table 6.1 - Acoustic impedance measurements of PAA samples compared with those simulated at $50 \mathrm{MHz}$.

\begin{tabular}{llll}
\hline Sample & $\begin{array}{l}\text { Porosity } \\
(\%)\end{array}$ & $\begin{array}{l}\text { Modelled Impedance } \\
\text { (MRayl) }\end{array}$ & $\begin{array}{l}\text { Measured Impedance } \\
\text { (MRayl) }\end{array}$ \\
\hline Type 1 & $25.5 \pm 2.5$ & $11-12$ & $13.3 \pm 2.00$ \\
Type 2 & $42 \pm 2.0$ & $8-9$ & $10.2 \pm 1.56$ \\
Type 3 & $48 \pm 2.0$ & $7-8$ & $9.06 \pm 1.39$ \\
\hline
\end{tabular}

Table 6.2 - Acoustic impedance measurements of PAA samples compared with those simulated at $100 \mathrm{MHz}$.

\begin{tabular}{llll}
\hline Sample & $\begin{array}{l}\text { Porosity } \\
(\%)\end{array}$ & $\begin{array}{l}\text { Modelled Impedance } \\
\text { (MRayl) }\end{array}$ & $\begin{array}{l}\text { Measured Impedance } \\
\text { (MRayl) }\end{array}$ \\
\hline Type 1 & $25.5 \pm 2.5$ & $11-12$ & $12.9 \pm 1.97$ \\
Type 2 & $42 \pm 2.0$ & $8-9$ & $10.3 \pm 1.58$ \\
Type 3 & $48 \pm 2.0$ & $7-8$ & $9.22 \pm 1.41$ \\
\hline
\end{tabular}

Table 6.3 - Acoustic impedance measurements of PAA samples compared with those simulated at $175 \mathrm{MHz}$.

\begin{tabular}{llll}
\hline Sample & $\begin{array}{l}\text { Porosity } \\
(\%)\end{array}$ & $\begin{array}{l}\text { Modelled Impedance } \\
(\text { MRayl) }\end{array}$ & $\begin{array}{l}\text { Measured Impedance } \\
\text { (MRayl) }\end{array}$ \\
\hline Type 1 & $25.5 \pm 2.5$ & $11-12$ & $13.1 \pm 2.00$ \\
Type 2 & $42 \pm 2.0$ & $8-9$ & $10.2 \pm 1.56$ \\
Type 3 & $48 \pm 2.0$ & $7-8$ & $9.19 \pm 1.41$ \\
\hline
\end{tabular}




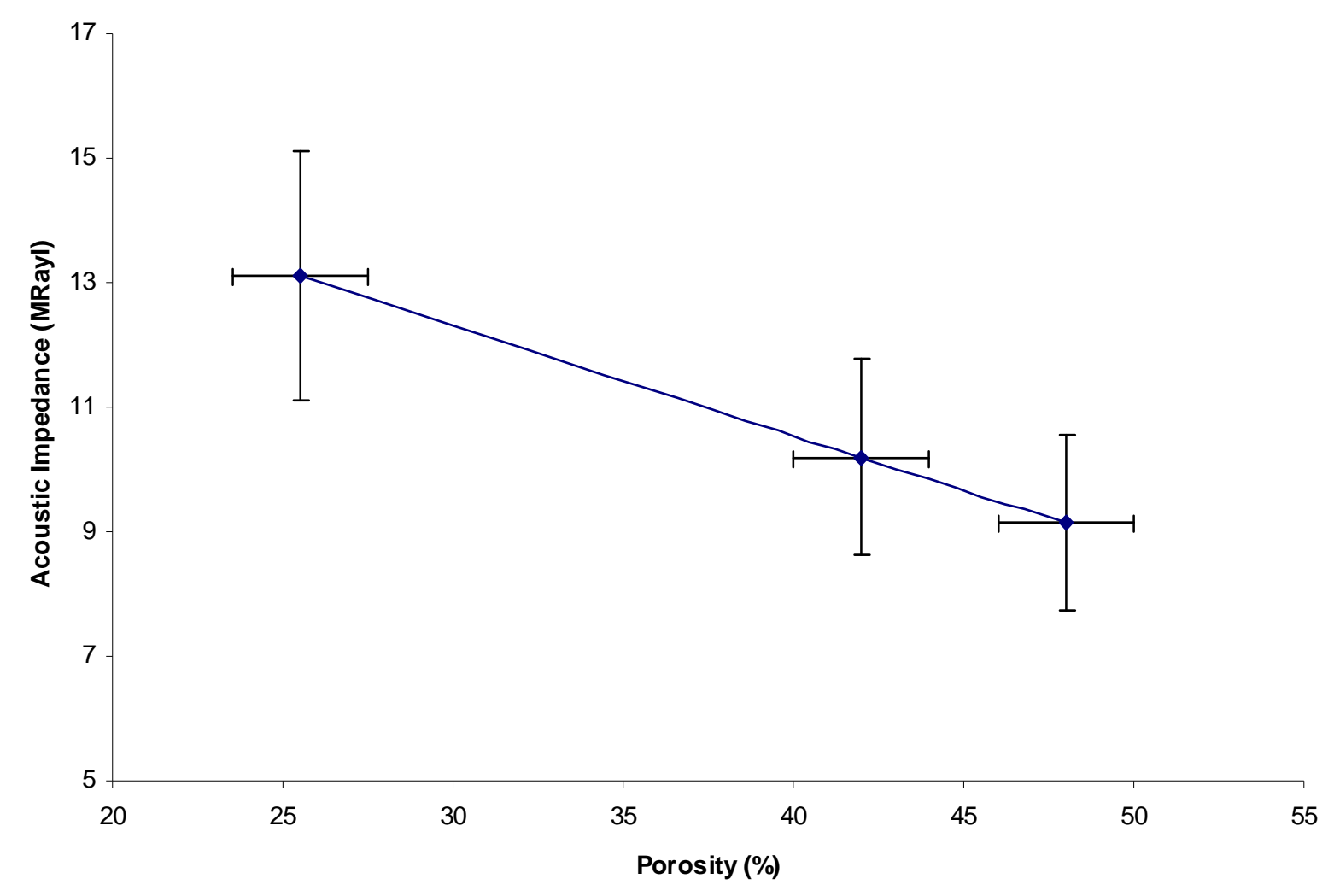

Figure 6.10 - Average acoustic impedance of PAA samples versus porosity.

The modelled impedances are those calculated in section 5.7.4 for the porosity ranges of the actual fabricated PAA samples. Comparing the modelled values with those measured experimentally they are in close agreement, with all of the measured values larger than those from the simulation. As with the velocity measurements, the acoustic impedance of alumina is larger than that of aluminium, and as a result the experimental measurements are also larger. The type 2 and type 3 measured impedances are very similar even though the samples possess very different dimensions. This is due to the porosities of these two samples being very similar, confirming the results from the modelling where it was found the acoustic impedance was independent of sample dimensions, architecture, and frequency, but dependent upon sample porosity. Figure 6.10 plots the average acoustic impedance of the PAA samples (impedance was found to be independent of frequency) as a function of the sample porosity. From this a linear relationship between acoustic impedance and porosity can be observed, which is in excellent agreement with that shown from the 3D modelling performed in section 5.7.4.

Assuming the linear relationship continues for higher porosities, since a measured experimental impedance of 9 MRayl could be realised for porosities of 46-50\% and the samples were still 
very robust, the porosity can be increased further to realise impedances of 7 MRayl or less. The PAA samples would then fulfil the very common range of impedances required for impedance matching layers of 7-12 MRayl.

It is recognised the acoustic impedances calculated from the modelling were found for a porous layer where the pores were void of any fluid. This is different to the experimental measurements where the pores were water filled since the samples were immersed during measurement. From the FEM modelling it was concluded the water loading of the samples should not cause a significant difference in impedance measurement, as the triangular and rectangular models showed no observable variation in velocity with water present. This is due to the majority of the energy propagating in the solid with very little energy transferred into the water.

\subsection{Attenuation}

The attenuation coefficients of the PAA samples were measured using the transmission method described in section 3.1.1.3. For each sample type two samples were fabricated, one approximately $100 \mu \mathrm{m}$ thick, the other at least close to $500 \mu \mathrm{m}$ thick in order to achieve a significant difference. Measuring the amplitudes after transmission through the two samples, the attenuation coefficient was calculated using equation (3.4). The same pulses and frequencies as described for the velocity and impedance measurements were used, with the measurements performed at normal incidence. The resulting attenuation measurements are summarised in Figure 6.11 expressed in $\mathrm{dBmm}^{-1}$. The measurements made were averaged over several samples. As with the velocity measurements, only a single waveform of significant amplitude was received corresponding to the fast wave travelling in the solid. This means all attenuation measurements refer to the fast wave travelling in the alumina.

Observing Figure 6.11, comparison between the three sample traces reveals significant differences in the attenuation coefficients. The samples with the smallest attenuation coefficients were the type 1 samples varying from $2.6 \mathrm{~dB} \cdot \mathrm{mm}^{-1}$ at $50 \mathrm{MHz}$ to just $8 \mathrm{~dB} \cdot \mathrm{mm}^{-1}$ at $175 \mathrm{MHz}$. The type 2 sample coefficients were slightly higher varying from $4.7 \mathrm{~dB} \cdot \mathrm{mm}^{-1}$ to $12 \mathrm{~dB} . \mathrm{mm}^{-1}$ over the same frequency range. The type 3 samples showed the highest attenuation coefficients varying from $11.6 \mathrm{~dB} \cdot \mathrm{mm}^{-1}$ to $22 \mathrm{~dB} \cdot \mathrm{mm}^{-1}$. 


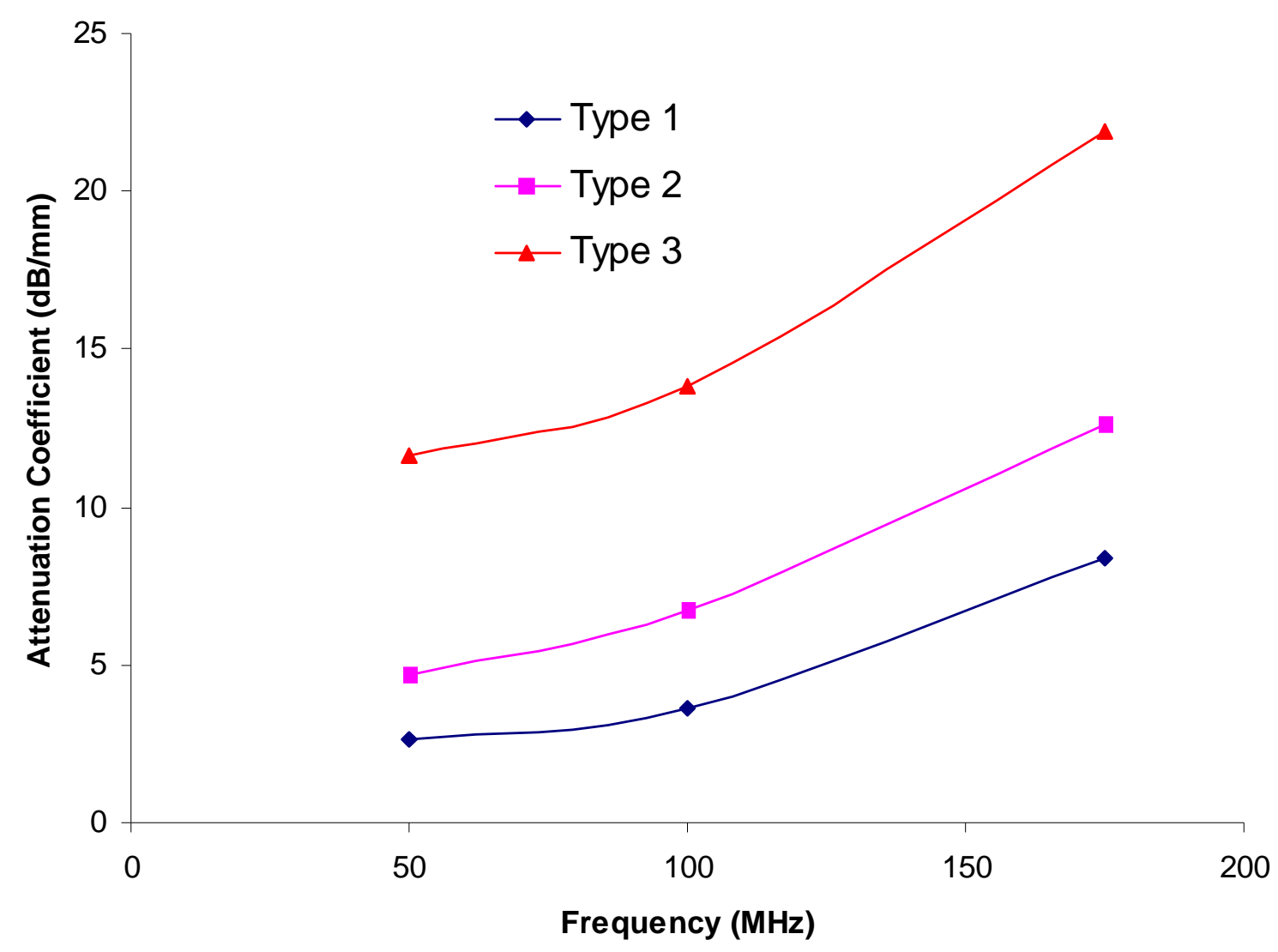

Figure 6.11 - Variations in PAA sample attenuation with frequency.

These variations can be explained by referring to the modelling in section 5.4. There, the energy partition between the aluminium pillars and the water pores of a porous aluminium layer was investigated for plane wave incidence. This was investigated by calculating the total energy propagating in the fast waves in the aluminium pillars relative to that propagating as slow waves in the water pores. Figure 5.14 revealed this ratio was influenced by the aluminium pillar to water pore width ratio. As the aluminium pillar widths were increased relative to the water pores (reducing porosity), the greater the amount of energy that propagated in the aluminium as a fast wave, thus reducing the total slow wave energy. For the type 3 samples possessing the largest porosities, the fast wave energy will be the smallest of the three samples, and the slow wave energy the largest. Since the slow wave is lost in the fast wave reverberation, and the attenuation measurement is based on the fast wave amplitude, this is consistent with Figure 6.11 where the attenuation is the highest in the type 3 samples. The difference in attenuation between the PAA samples is therefore a measure of modal energy distribution, not a difference in sample scattering or absorption. 
To understand the significance of the above attenuation coefficient measurements requires comparison with the currently used state of the art matching layers made from nanoparticle loaded epoxies as found in [101] and [103]. Reference [101] uses alumina colloidal/polymer nanocomposite films with particle sizes in the range of 10-40 nm. The lowest attenuation measurement achieved from this work for any of the samples was $15 \mathrm{dBmm}^{-1}$ at a frequency of $40 \mathrm{MHz}$, and appeared to increase beyond this with the fourth power of frequency. Comparison with our samples reveals even the type 3 samples show almost half as much attenuation at $50 \mathrm{MHz}$ varying with the square of frequency. Reference [103] used nanocomposite cerium oxide polymer matching layers with particle sizes of $20 \mathrm{~nm}$, reporting an attenuation

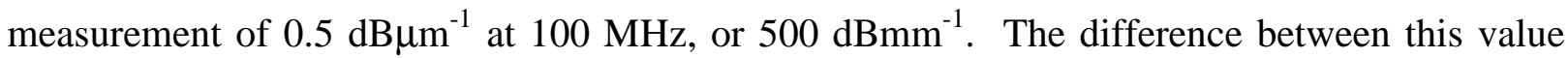
and the largest value measured for our samples at $100 \mathrm{MHz}$ of $11 \mathrm{dBmm}^{-1}$ is extremely significant. For frequencies in excess of $100 \mathrm{MHz}$ these nanoparticle matching layers will become excessively lossy, making our porous samples a very suitable and desirable alternative.

The excellent attenuation performance of the PAA samples is due to the highly collinear nanostructured alumina walls. These extend through the samples from top to bottom, acting as waveguides to the propagating waves throughout the entire structure. These waveguides cause no scattering to the propagating waves, with the only attenuation caused by material absorption. This is a significant improvement over the nanometer-sized powders used in [101] and [103] to make current matching layers, which result in significant amounts of frequency dependant scattering in addition to the material absorption.

\subsection{Summary of Measured PAA Sample Properties}

The experimental data presented in the previous sections 6.1, 6.2, and 6.3 for each of the inhouse fabricated PAA samples are summarised in Table 6.4. Since the velocity and acoustic impedance measurements were found to be frequency independent, the values summarised in the table below are averaged over the results from the three frequencies used. The attenuation coefficient measurements being dependent upon frequency are stated for all three frequencies.

The type 1 samples possess 1) the smallest physical dimensions and porosity, 2) the largest velocity and acoustic impedance, but 3 ) the smallest attenuation coefficients. Type 3 samples are the opposite possessing the largest physical dimensions and porosity, and in terms of the 
Table 6.4 - Measured in-house fabricated PAA sample properties.

\begin{tabular}{|c|c|c|c|c|c|c|c|}
\hline $\begin{array}{l}\text { Sample } \\
\text { Type }\end{array}$ & Electrolyte & $\begin{array}{l}\text { Pore Size } \\
(\mathrm{nm})\end{array}$ & $\begin{array}{l}\text { Pore } \\
\text { Spacing } \\
(\mathrm{nm})\end{array}$ & $\begin{array}{l}\text { Porosity } \\
(\%)\end{array}$ & $\begin{array}{l}\text { Velocity } \\
\left(\mathrm{ms}^{-1}\right)\end{array}$ & $\begin{array}{l}\text { Acoustic } \\
\text { Impedance } \\
\text { (MRayl) }\end{array}$ & $\begin{array}{l}\text { Attenuation } \\
\text { (dB.mm }{ }^{-1} @ \\
\text { MHz) }\end{array}$ \\
\hline \multirow[t]{3}{*}{1} & Sulphuric & 30 & 60 & $25.5 \pm 2.5$ & $5840 \pm 43$ & $13.1 \pm 2.00$ & $2.11 @ 50$ \\
\hline & & & & & & & $3.45 @ 100$ \\
\hline & & & & & & & $8.55 @ 150$ \\
\hline \multirow[t]{3}{*}{2} & Oxalic & 40 & 80 & $42 \pm 2$ & $5696 \pm 41$ & $10.2 \pm 1.56$ & $3.67 @ 50$ \\
\hline & & & & & & & $5.80 @ 100$ \\
\hline & & & & & & & $12.1 @ 150$ \\
\hline \multirow[t]{3}{*}{3} & Phosphoric & 400 & 500 & $48 \pm 2$ & $5671 \pm 40$ & $9.16 \pm 1.40$ & $8.86 @ 50$ \\
\hline & & & & & & & $11.1 @ 100$ \\
\hline & & & & & & & $18.7 @ 150$ \\
\hline
\end{tabular}

mechanical properties the smallest velocity and acoustic impedance, but the largest attenuation coefficient. The type 2 samples are a combination of the others possessing physical dimensions almost the same as the type 1 samples, yet possess porosities, architectural, and mechanical properties much closer to the type 3 samples. This is due to the architecture and porosity appearing to determine the mechanical properties of the samples.

All three types of samples show significant advantages over currently used loaded epoxy materials. The normalising properties of the PAA samples allows for reception over a much wider range of angles, resulting in increased signal to noise ratio, and reception of waves that would have otherwise gone critical. The attenuation coefficients of these samples are excellent out to and beyond frequencies where the attenuation of currently used layers become unacceptably high. The PAA matching layers showed no measurable dispersion at least to 200 $\mathrm{MHz}$ preserving waveshape and frequency content. The variation in PAA impedance with porosity allows them to be tailored for a specific application, and if porosities of $60 \%$ (realistic for these PAA samples) can be fabricated, will result in a potential impedance range of 5-17 MRayls. 


\section{Chapter 7}

\section{Anisotropic Poly(Vinyl-Alcohol) Tissue Mimicking Phantoms}

Tissue mimicking phantoms are an important component in medical ultrasound research, as they often offer a more repeatable, easier to prepare, and ethical alternative to handling actual tissue samples. Such phantoms should possess similar properties, structures, and dimensions to the real tissues of interest and it should be possible to easily replicate these phantoms to ensure consistency throughout many experiments.

Poly (vinyl alcohol), commonly known as PVA, is now the accepted material used by medical ultrasound researchers to make tissue phantoms. PVA is currently most commonly made into homogeneous phantoms known as cryogels that are covered extensively in the literature [113][116]. These gels maintain their shape at room temperature and can be tailored to possess similar tissue properties e.g. velocity, by exposing the samples to repeated freezing-thawing cycles. However as shown in [113] the cross-linking process that takes place during the freezethaw cycles does not introduce any preferred orientation or significant anisotropic structure (at least at the micron scale) into the phantom. As described in chapter 1, this is significantly different from many real tissues such as muscle that possess a highly anisotropic structure, containing fibres microns in diameter, and tens of microns in length, orientated along the same direction. 
As medical ultrasound systems continue to increase towards the use of $100 \mathrm{MHz}$ and higher frequencies, these real tissue properties and dimensions need to be considered, since the insonifying wavelengths become comparable to the tissue microstructure dimensions. Such microstructure can influence the wave velocity, scattering, and propagating modes depending on the frequency-microstructure dimensions and orientation. New more advanced tissue phantoms that possess these real tissue structures and dimensions need to be developed for this purpose.

Microstructured anisotropic PVA phantoms were developed in this work that possess tailorable acoustic properties with similar dimensions, microstructure, and orientation to that of real fibrous tissues. These phantoms appear to be a suitable starting point for the fabrication of more advanced tissue-mimicking phantoms.

\subsection{The Freeze Casting Process}

A process known as freeze casting was used to fabricate porous anisotropic tissue phantoms. Materials with aligned porous structures have many applications in areas such as organic electronics, ceramics, microfluidics and molecular filtration. These materials can be fabricated using processes such as microfabrication and photolithography, however a simple, cheap, and novel approach is to use freeze casting or directional freezing. Using this simple technique, aligned porous materials with 2D surface structures or 3D monolithic structures can be made. The same basic technique can be applied to produce porous ceramic structures [117], [118], porous polymer structures [119], [120], or drug release scaffolds [121] for controlled drug delivery.

The freeze casting process used in this work was tailored for this application and consisted of four main steps:

1. Slurry preparation

2. Unidirectional freezing

3. Sample preparation

4. Freeze drying

Each of these steps is described in sections 7.1.1, 7.1.2, 7.1.3, and 7.1.4 respectively below. 


\subsubsection{PVA Slurry Preparation}

The first step in the process was the preparation of aqueous PVA slurry. The choice of PVA polymer is very important in determining the final structural properties of the phantom. Firstly, the molecular weight of the PVA polymer determines the solubility of the samples with the smaller molecular weights being the more soluble. Samples prepared using lightweight polymers were observed to dissolve in a matter of seconds. Molecular weight was also found to contribute to the final mechanical strength of the samples by contributing to the degree of PVA inter- and intra- molecular bonds in the phantom. The PVA used in this work was purchased from Sigma-Aldrich, catalogue number 563900, batch \# 10708CH, possessing an average molecular weight of $130,000 \mathrm{gmol}^{-1}$ and was greater than $99 \%$ hydrolysed. For this molecular weight the samples lasted 2-3 days from the time of immersion in water till fully dissolved. In this work, once a sample was immersed in water, measurements were performed immediately to minimise the affects of sample degradation over time on the measurement accuracy.

PVA slurry concentration was found to influence the final porosity of the sample, pore wall thickness, and density. The PVA slurries were varied between 5-10\% concentrations by weight by slowly adding 5-10 g of PVA powder to 90-95 $\mathrm{mL}$ of pure water (depending on concentration) in a beaker that was heated to $80{ }^{\circ} \mathrm{C}$. The solution was then maintained at $80{ }^{\circ} \mathrm{C}$ and mixed thoroughly using an automatic heater/stirrer for 2-3 hours to ensure a uniform slurry was obtained. To minimise dehydration during the process, the beaker was covered with plastic wrap. Silica gel particles - often added as a scattering component to homogeneous PVA phantoms - are not required as scattering is intrinsic to these samples due to their porous microstructure.

\subsubsection{Unidirectional Freezing}

Once the slurry was prepared, the next step was the unidirectional freezing of the slurry in order to create an aligned porous microstructure. $4 \mathrm{~mL}$ of the solution was poured into a $5 \mathrm{~mL}$ polystyrene flat base vial. It is very important to use a vial with minimal curvature and defects on the base as these factors can introduce unwanted nucleation sites, biasing the microstructure formation. This vial was then placed into a sample holder attached to the Thorlabs 3-axis positioning stage (described in section 3.5) as shown in Figure 7.1. Larger volumes can be used. 


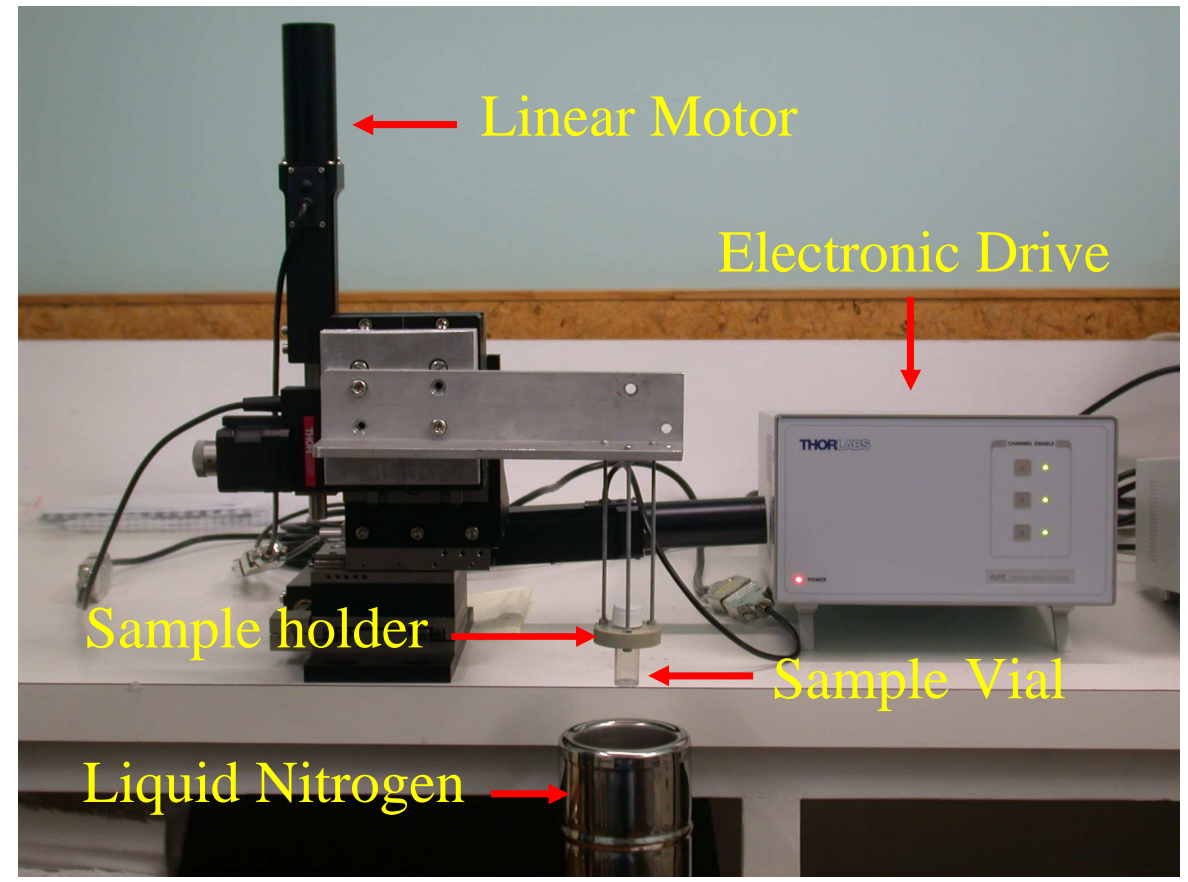

Figure 7.1 - PVA sample vial lowering setup.

The sample vial was then lowered by the z-axis linear actuator at a rate between $3-7 \mathrm{~mm} / \mathrm{minute}$ into a low thermal conductivity flask of liquid nitrogen. The low thermal conductivity flask was used to prevent nitrogen evaporation. In order to ensure unidirectional freezing, the sample holder must be held rigidly and completely isolated from all movement. This will ensure that the temperature gradient from liquid nitrogen temperature at the base of the sample vial, to room temperature at the top of the sample vial, only exists along the direction of lowering. The top of the liquid nitrogen flask where the sample is lowered through is surrounded by polystyrene (not shown) to further prevent liquid nitrogen evaporation. Evaporation results in the effective rate of lowering being slower than the actual set value.

The freeze-casting process that takes place during lowering is described with the aid of Figure 7.2. As the sample is first lowered into the liquid nitrogen, ice nucleation occurs on the bottom of the vial as the water content in the slurry begins to freeze, and the ice crystals in the sample form according to the Mullins-Sekerka instability criterion [120]. This criterion defines a wavelength that determines the periodicity of the ice cell growth, dependent on many different parameters. 


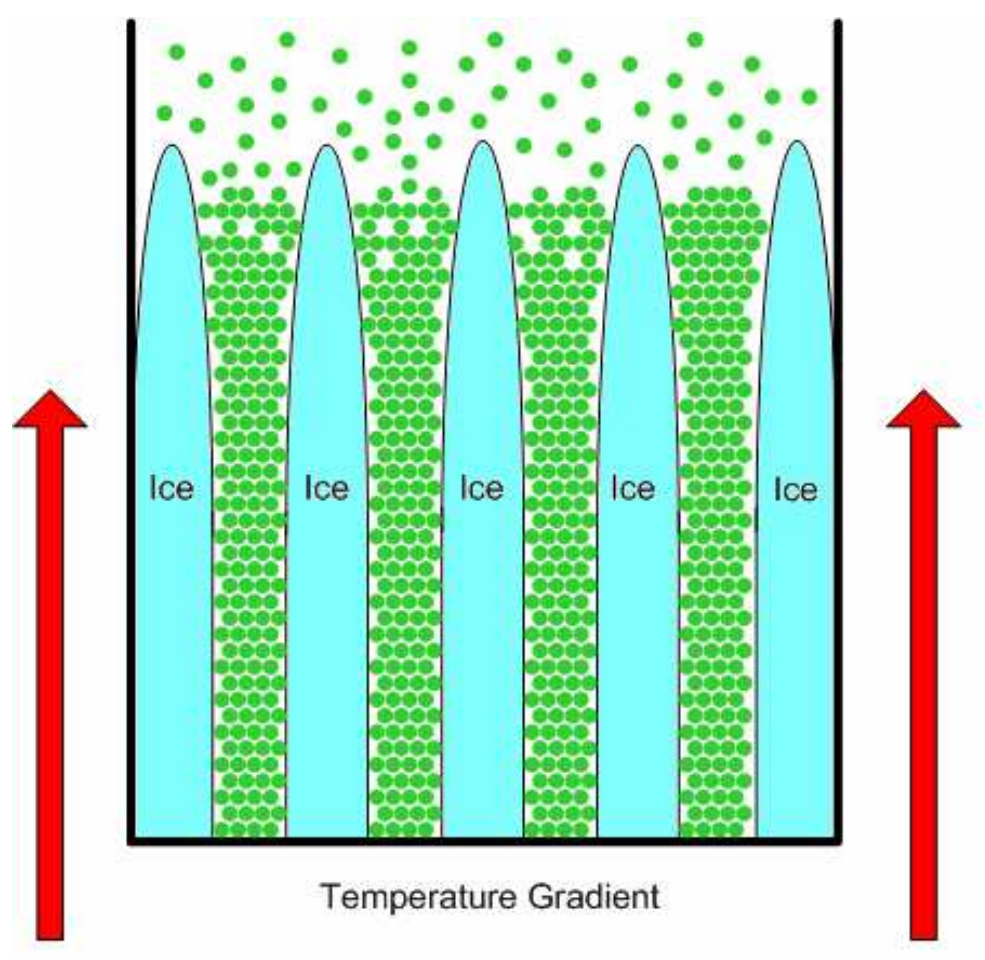

Figure 7.2 - The freeze casting process showing the ice crystal formation within the slurry vial that is lowered into the liquid nitrogen.

With the first ice growth sites separated by the Mullins-Sekerka wavelength, these original sites become preferred formation sites for further ice growth, leading to the formation (assuming a unidirectional temperature gradient is present) of vertical ice pillars along the direction of freezing. The PVA molecules are excluded from the ice formation process, causing them to accumulate in between the ice pillars. The PVA polymer chains then form tight inter and intramolecular bonds with surrounding polymers trapped between the ice pillars. This process continues to take place until the sample is fully immersed ensuring the sample is fully frozen. The sample was then raised at a much faster rate, removed, and then placed into an external container of liquid nitrogen to prevent thawing of the sample before sample preparation.

\subsubsection{Sample Preparation}

Sample preparation involved cutting the sample to the desired thickness before the final freezedrying step. Once freeze dried, the PVA samples were almost impossible to cut without causing permanent damage. Therefore the easiest solution was to cut the samples to size whilst still frozen. After evaluating several different methods, samples were cut by sawing them while 
immersed in liquid nitrogen. Sawing caused the least surface structural damage, generating no significant heat provided the blade was kept cool.

The plastic vial with the sample still inside was clamped in a custom made PVC plastic v-block that was mounted inside a small volume plastic box; PVC was used to reduce nitrogen boil off. The box was then filled with liquid nitrogen, immersing the sample for sawing. Sawing was carried out using an Emco mill drill machine mounted at 90 degrees with a slotting saw blade that was lowered to perform the cut. The slotting saw blade was cooled before sawing to minimise initial heat exposure to the PVA samples.

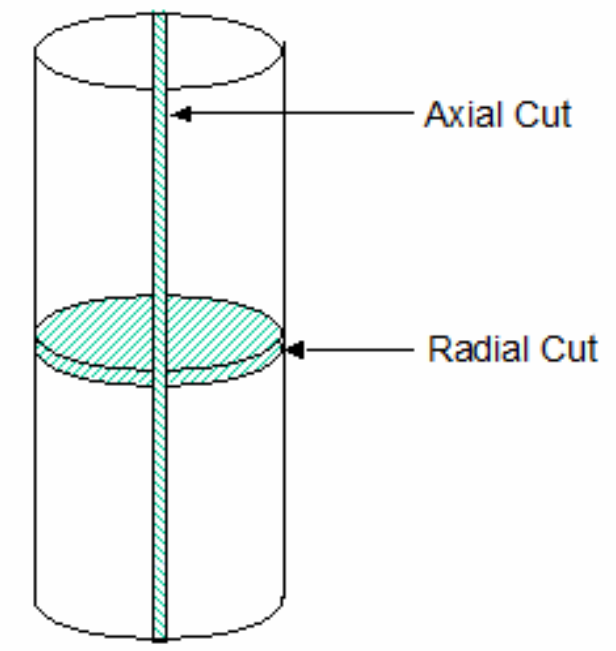

Figure 7.3 - Axial and radial cuts made on frozen PVA samples.

To cut samples for measurement along the direction of PVA alignment, the first cut was made halfway along the sample vial, perpendicular to the direction of casting i.e. radially as shown in Figure 7.3. Successive radial cuts were then made on each sample half from the middle outwards, cutting the sample into discs of 1-2 mm in thickness and diameter of $10 \mathrm{~mm}$. The diameter of the samples was set by the diameter of the vial, in this case $10 \mathrm{~mm}$. Once cut, these discs were then placed back into liquid nitrogen until the final freeze-drying process. To cut samples for measurements perpendicular to the direction of PVA alignment, the first cut was made axially as shown in Figure 7.3, splitting the sample along the axis of the vial. Cuts were then made radially on each half, and once again samples were immediately placed in liquid nitrogen for temporary storage. 


\subsubsection{Freeze Drying of Prepared Discs}

Once the solid anisotropic discs had been prepared, they were still not useable since leaving the sample at room temperature would cause the samples to thaw and eventually lose microstructure. By removing the ice crystals, these phantoms (provided these samples were not immersed in water) could be kept at room temperature for many months. Therefore the ice crystals had to be removed without letting the samples thaw. To do this the process of freezedrying [122] was used that removes the ice crystals via sublimation. This process is a standard procedure commonly used in the food industry for long-term food preservation. Freeze drying is a thermodynamic process that transforms water from the solid ice phase to the gas phase without passing through the liquid phase. Figure 7.4 shows the phase diagram for pure water. The process of freeze-drying (or sublimation) represented by the left most arrow requires the surrounding pressure of the water to be reduced and heat applied. However, if the pressure is reduced sufficiently, no heating is required. This is the concept of a commercial flask freeze dryer commonly used in chemistry laboratories where the pressure is reduced below 300 mTorr.

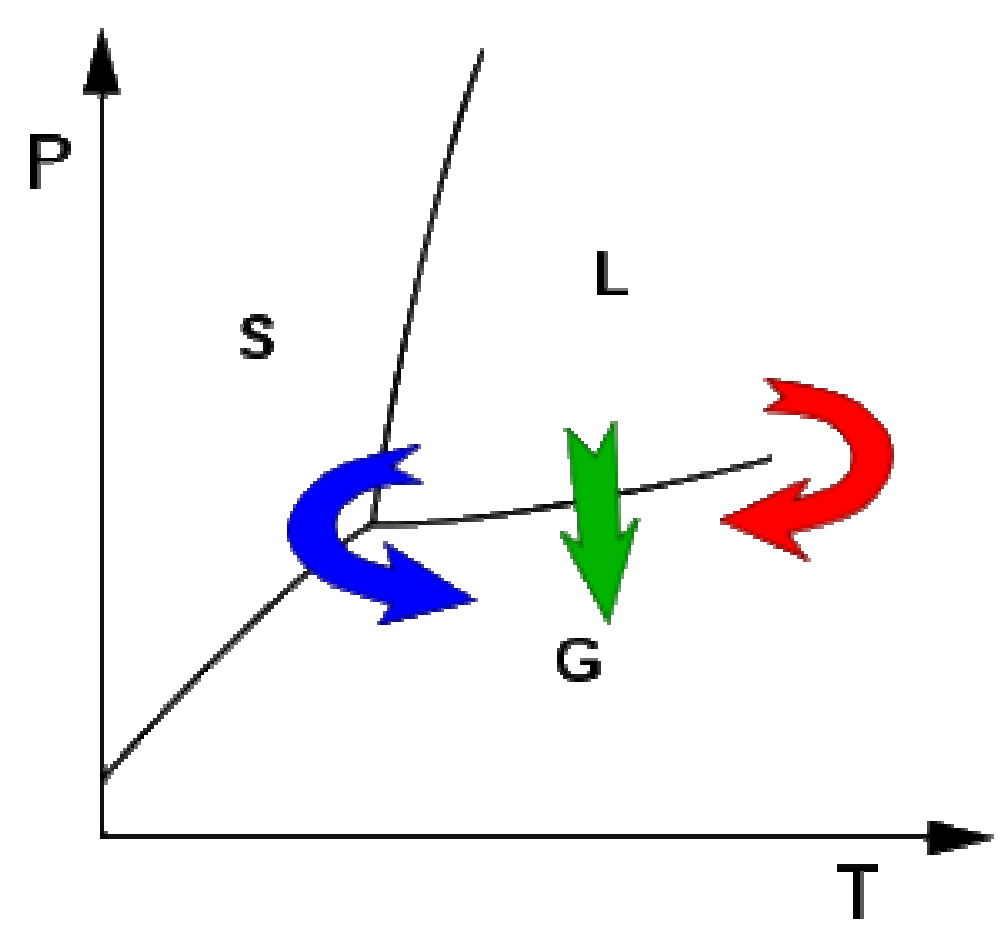

Figure 7.4 - Pressure/temperature phase transition diagram for pure water. 
A custom vacuum chamber was prepared that was evacuated using a double vane rotary pump. This pump is capable of reducing the chamber below 250 mTorr with several samples present, eliminating the need for any heating. Commercial freeze dryers contain a condenser, converting the water vapour into the liquid phase to reduce corrosion of the pump vanes and preventing water vapour being absorbed into the pump oil. A condenser was not used in our setup due to the small volumes of water involved. Regular pump ballast was applied after each freezedrying process to remove any water vapour absorbed, and the pump oil was changed regularly as a precaution. Samples were fabricated and cut to size during the day, then placed in the freeze dryer and left overnight.

\subsection{PVA Structure}

Using the fabrication method described in the previous section, example horizontal and longitudinal cross sections of the PVA samples are presented. These structures were viewed using a JEOL 5300 scanning electron microscope (SEM). The samples were prepared for SEM viewing by sputtering a $30 \mathrm{~nm}$ gold film onto the viewing surface. The samples were attached to the SEM sample mount using carbon tape from the bottom of the mount to the top of the sample ensuring good electrical grounding of the material.

Figure 7.5 shows a SEM micrograph of the horizontal cross section of a phantom prepared using a $5 \%$ concentration by weight PVA slurry, lowered at a nominal rate of $3 \mathrm{~mm} / \mathrm{min}$. The significant anisotropic microstructure of the phantom can clearly be observed. The pores of the sample are created by the absence of the ice crystals that were sublimed off during the freeze drying process and are arranged in a fairly regular structure. The pores are almost hexagonal in shape and relatively consistent in size. Even though this is a horizontal cross section, intended to be looking directly down the pores, the sample looks as though it was on an angle when the image was taken. This is due to a slight difference in angle between the SEM viewer and the sample vertical pore axis. The pores also appear to be asymmetrically shaped, as though the sample had undergone some compression, resulting in elongated hexagonal pores. This was present in a number of samples, and it is quite possible that during the application of the carbon tape to the sample, slight compression may have taken place due to handling of the samples. 


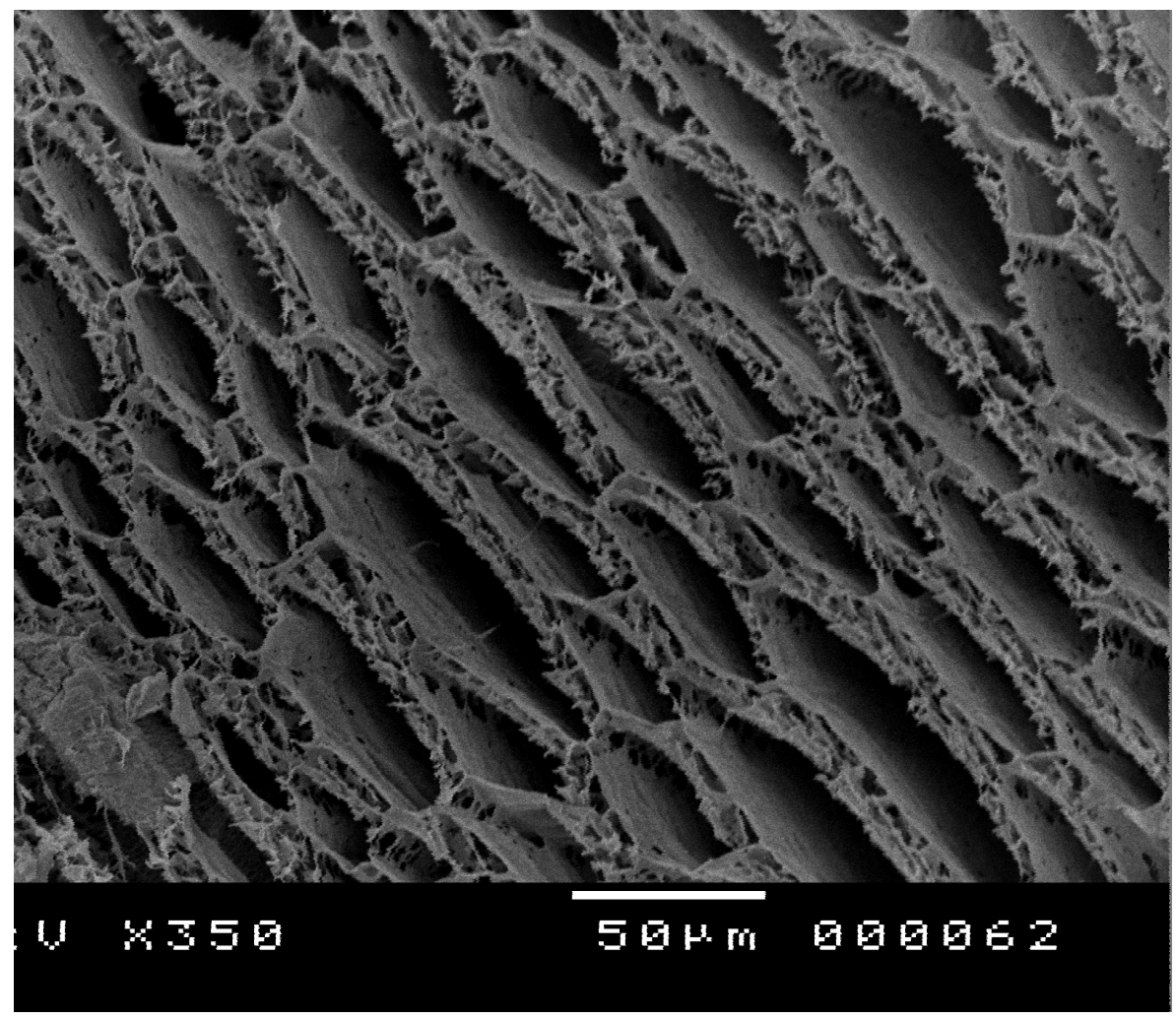

Figure 7.5 - Horizontal cross section (section made perpendicular to the direction of freeze casting) of a $5 \%$ concentration by weight PVA phantom lowered at $3 \mathrm{~mm} / \mathrm{min}$.

The PVA walls separating the pores are of consistent thickness with dimensions between 5-15 $\mu \mathrm{m}$. These pore wall dimensions are in good agreement with the myocardial fibre diameters measured in rat heart tissue, found to be $10-12 \mu \mathrm{m}$ in diameter as stated in [123]. The pore walls themselves can be observed to possess their own microstructure. Increasing the PVA concentration or reducing the sample-lowering rate increases the density of these walls. The solid feature located in the bottom left corner disrupting the regularity of the structure is not part of the actual phantom microstructure. Instead, this is a flake that has settled on the surface as a result of fracturing the original sample for SEM imaging.

Figure 7.6 shows a SEM micrograph of the longitudinal cross section from the same phantom. Once again the anisotropic nature of the phantom is observed. From this image it can be seen the phantom possesses a well-defined orientation and significant anisotropy, with regularity much like that found in fibrous tissues. The pore walls appear to run vertically through the sample along the direction of freeze casting, in the case of Figure 7.6 from the bottom left of the image to the top right. Confirmation that the pores form along the direction of freeze-casting was found in the experimental velocity measurements (see section 7.3). 


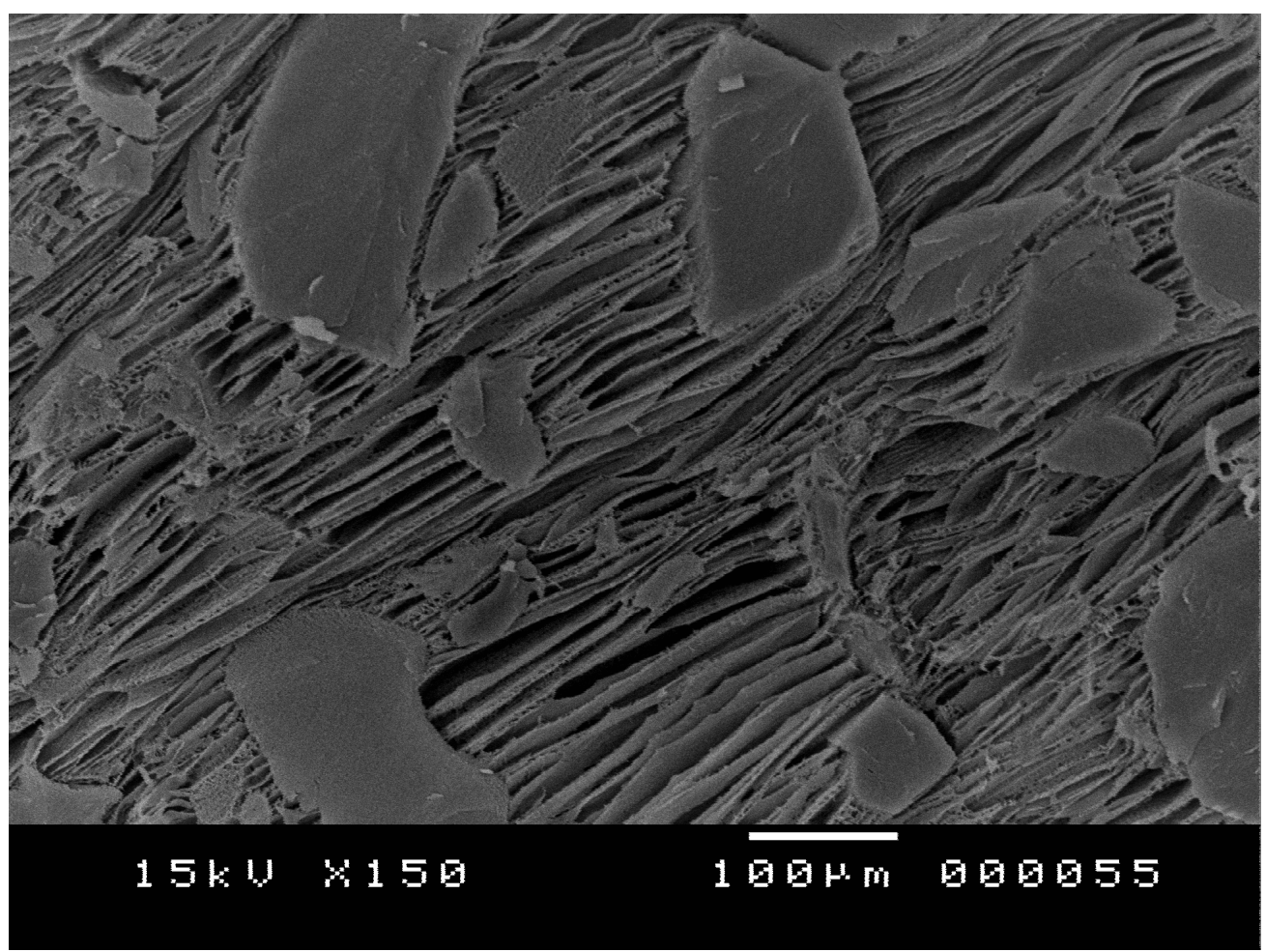

Figure 7.6 - Longitudinal cross section (section made parallel to the direction of freeze casting) of a $5 \%$ concentration by weight PVA phantom lowered at $3 \mathrm{~mm} / \mathrm{min}$.

The large surface structures observed are not part of the microstructure but again flakes that have settled on the sample during SEM preparation. As observed in Figure 7.5 the dimensions of the pore walls are approximately $10 \mu \mathrm{m}$ on average, in good agreement with real fibrous tissue dimensions. The pore widths can be observed to vary somewhat, and do not necessarily appear to be the same size as those observed in Figure 7.5. Again, a small error in viewing angle may have occurred so that viewing exactly perpendicular to the pore axis was not occurring. These observations though are more likely a consequence of the sample preparation process. When vertical sections of the samples are prepared, the samples lose their strength, potentially causing folding of the pore walls. Also, during preparation the sample cut will not be such that it splits the pores directly in half, and as a result gives the appearance of smaller pores. Finally, pores are not exactly in line with each other so some pores will appear larger, some smaller, and some the same as those observed in Figure 7.5.

The rate at which the sample is lowered into the liquid nitrogen greatly influences the pore spacing (the size of the ice crystals formed). Slow freezing rates result in groups of large ice crystals. As a result the accumulation of PVA between ice pillars becomes denser for these slower rates, creating more rigid pore walls and mechanical strength. 


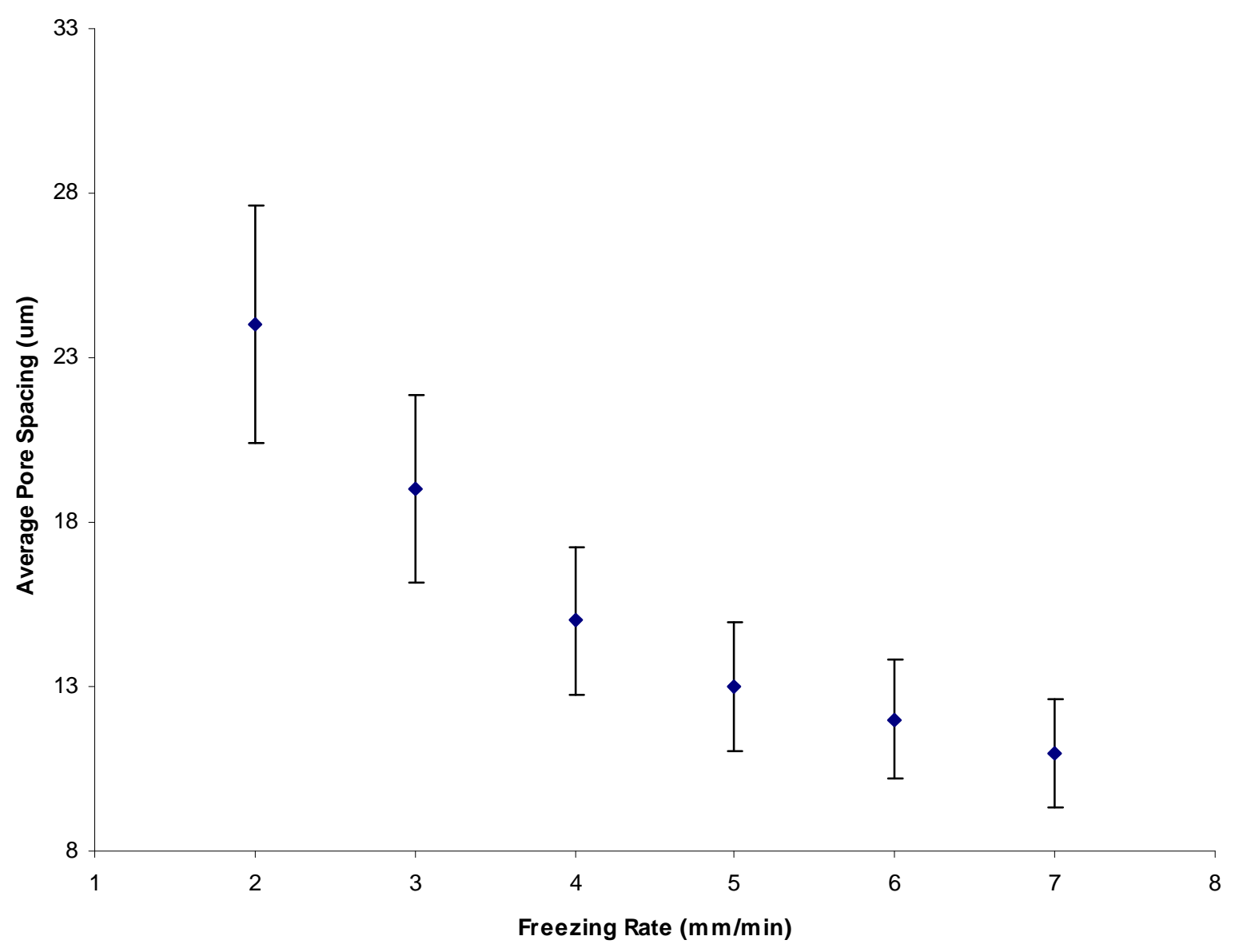

Figure 7.7 - Average pore size versus freezing rate for $5 \%$ by weight PVA concentration.

Figure 7.7 shows the average pore spacing for $5 \%$ by weight PVA slurry concentration phantoms, lowered at five different freezing rates. Values for each of the plotted freezing rates were based on several SEM images with the pore sizes measured and averaged for each. For elongated pores both the length and widths were measured and the average of the two found. As can be observed a reasonable range of pore spacing can be achieved by lowering the samples at different rates. For faster freezing rates, the ice does not have time to form large crystal groups, and instead the ice crystal groups are smaller in size resulting in smaller phantom pores. The PVA pore walls for these freezing rates become less dense. Finally, for freezing rates greater than $10 \mathrm{~mm} / \mathrm{min}$, it was found the regularity in ice crystal spacing and long distance ice formation was lost. Too fast a freezing rate causes the initial ice crystals to be randomly spaced with no grouping. These randomly distributed crystals become the preferred formation sites for further ice growth, but with little chance for ice to form on these sites, these new ice crystals will also be loosely ordered, resulting in an overall poorly ordered amorphous ice structure. 
For $5 \%$ PVA concentration, the average pore spacing is observed to vary between $10-25 \mu \mathrm{m}$ for freezing rates between $2-7 \mathrm{~mm} / \mathrm{min}$. For freezing rates greater than $7 \mathrm{~mm} / \mathrm{min}$ there is very little decrease in the pore spacing before irregular ice crystal formation occurs for rates greater than $10 \mathrm{~mm} / \mathrm{min}$. Instead, to further reduce the average pore spacing the concentration of the PVA can be increased.

In addition to the above process parameters, it is proposed by introducing a nucleation plate within the sample container prior to cryogenic cooling, the seed layer can be controlled and hence the overall resulting microstructure influenced accordingly. The nucleation plate could be placed at the bottom of the slurry container containing microfabricated wells with dimensions of the desired crystalline structure, allowing phantom properties to be more rigorously controlled. This could lead to the fabrication of more advanced structured phantoms.

\subsection{Velocity Measurements}

Measurements of the velocity through the PVA phantoms are presented here. These were made using the transmission measurement method described in section 3.1.1.2. This method requires the thickness of the PVA sample to be known. Since these samples are not solid, they were measured using the non-destructive transmission method described in section 3.1.1.1.

To measure the anisotropic characteristics of these phantoms, the velocity measurements were made both parallel and perpendicular to the freeze casting direction. Numerous velocity experiments on various fibrous tissues described in the literature find the orientation of the fibres relative to the incident waves significantly influence the propagating wave velocity [64], [124]. However, the velocity frequency dependence in these papers is found to be very low showing very little dispersion across a wide bandwidth.

Several phantoms were used for the following measurements with the results averaged. The phantoms were made from a $7 \%$ concentration by weight PVA slurry and were lowered at a nominal rate of $5 \mathrm{~mm} / \mathrm{min}$. Samples were cut to approximately $1 \mathrm{~mm}$ in thickness to obtain an accurate measurement. 


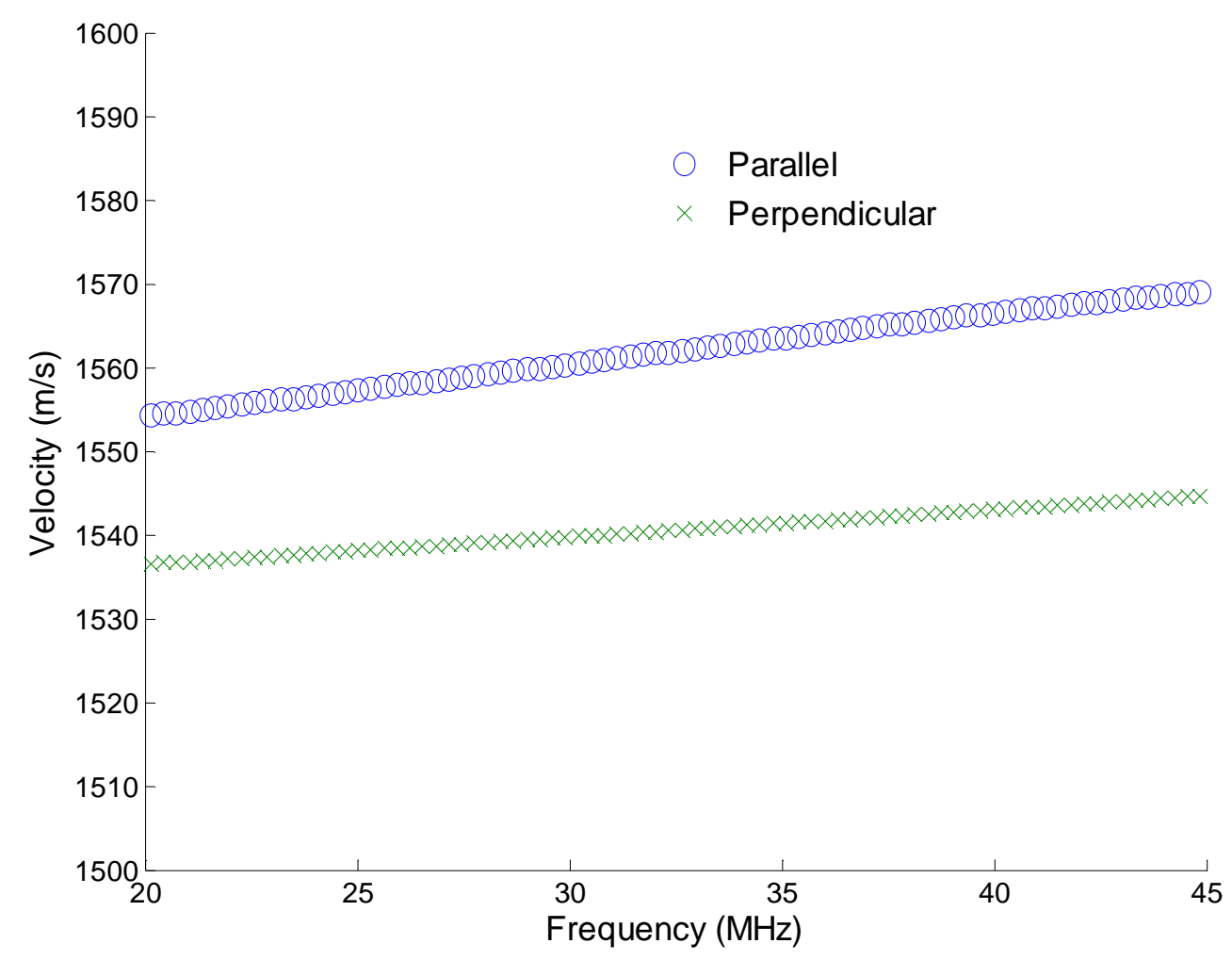

Figure 7.8 - Phantom velocity variations with frequency for a $7 \%$ concentration by weight PVA phantom measured parallel (circles) and perpendicular (squares) to the direction of freeze casting.

The resulting measurements of the acoustic velocity parallel and perpendicular to the direction of PVA alignment are both plotted in Figure 7.8. The plots confirm the results from the literature where the velocities measured parallel to the PVA alignment (along the freeze casting direction) are greater than those measured perpendicular. This confirms the pores form along the freeze casting direction since the larger velocities indicate greater mechanical strength due to the increased PVA alignment. At $20 \mathrm{MHz}$ the difference between the two is approximately $20 \mathrm{~ms}^{-1}$ and by $45 \mathrm{MHz}$ this gap has increased to approximately $30 \mathrm{~ms}^{-1}$. This is due to the measurements made parallel to the PVA orientation increasing at a faster rate of $0.6 \mathrm{~ms}^{-1} \mathrm{MHz}^{-1}$ compared to the measurements made perpendicular increasing at just over half the rate of $0.36 \mathrm{~ms}^{-1} \mathrm{MHz}^{-1}$. These results demonstrate very little dispersion and are in good agreement with those measured in [124] for lamb heart myocardium. Myocardium is one of the highly anisotropic fibrous muscle tissues found in the heart as observed in images from [125]. In that work the rate of dispersion measured along the direction of fibre orientation was $0.74 \mathrm{~ms}^{-1} \mathrm{MHz}^{-1}$, significantly greater than the rate measured perpendicular of $0.24 \mathrm{~ms}^{-1} \mathrm{MHz}^{-1}$. 
The values of the velocities are also in good agreement with those measured in [64] and [126]. Reference [64] contains measurements for bovine heart muscle tissue over the frequency range of 20-40 MHz measured along the direction of fibre alignment. For this frequency range the velocities varied from 1565-1570 $\mathrm{ms}^{-1}$ compared to the measured PVA velocities of $1555-1565 \mathrm{~ms}^{-1}$. The phantom velocities are not limited to these values and can be varied significantly up or down to resemble other tissues by varying the various process parameters.

\subsection{Attenuation Measurements}

The attenuation coefficient of the PVA samples was calculated using the transmission method described in section 3.1.1.3, averaged over several measurements. This method required two samples of near identical microstructure for an accurate measurement. To achieve this, for each separate attenuation measurement two samples were used that were cut from the same vial. One sample was cut to no greater than $0.5 \mathrm{~mm}$ in thickness and the other $1 \mathrm{~mm}$ ensuring a reasonable thickness difference for the calculation. Sample thickness was again measured nondestructively using the transmission method described in section 3.1.1.1.

The attenuation coefficients were measured for several phantoms made from a $7 \%$ by weight concentration PVA slurry lowered into liquid nitrogen at a nominal rate of $5 \mathrm{~mm} / \mathrm{min}$. The results were averaged and are plotted in Figure 7.9 over a frequency range of 20-45 MHz. These measurements were performed along the direction of PVA alignment and expressed in $\mathrm{dBmm}^{-1}$. The PVA phantom measurements are plotted in Figure 7.9 as blue circles. The figure also includes measurements that were performed on normal human myocardium tissue parallel to the direction of fibre alignment obtained from [127]; these are plotted as filled green squares.

Both data sets show monotonically increasing functions. The attenuation coefficient at $20 \mathrm{MHz}$ was approximately $5 \mathrm{dBmm}^{-1}$ for the PVA increasing to $13 \mathrm{dBmm}^{-1}$ at $45 \mathrm{MHz}$. The PVA measurements are in excellent agreement with the myocardium measurements over the plotted frequency range, varying proportionally to the 1.6 power of frequency. This exponent value is also in good agreement with the value measured from [64] of 1.56 for bovine heart muscle. 


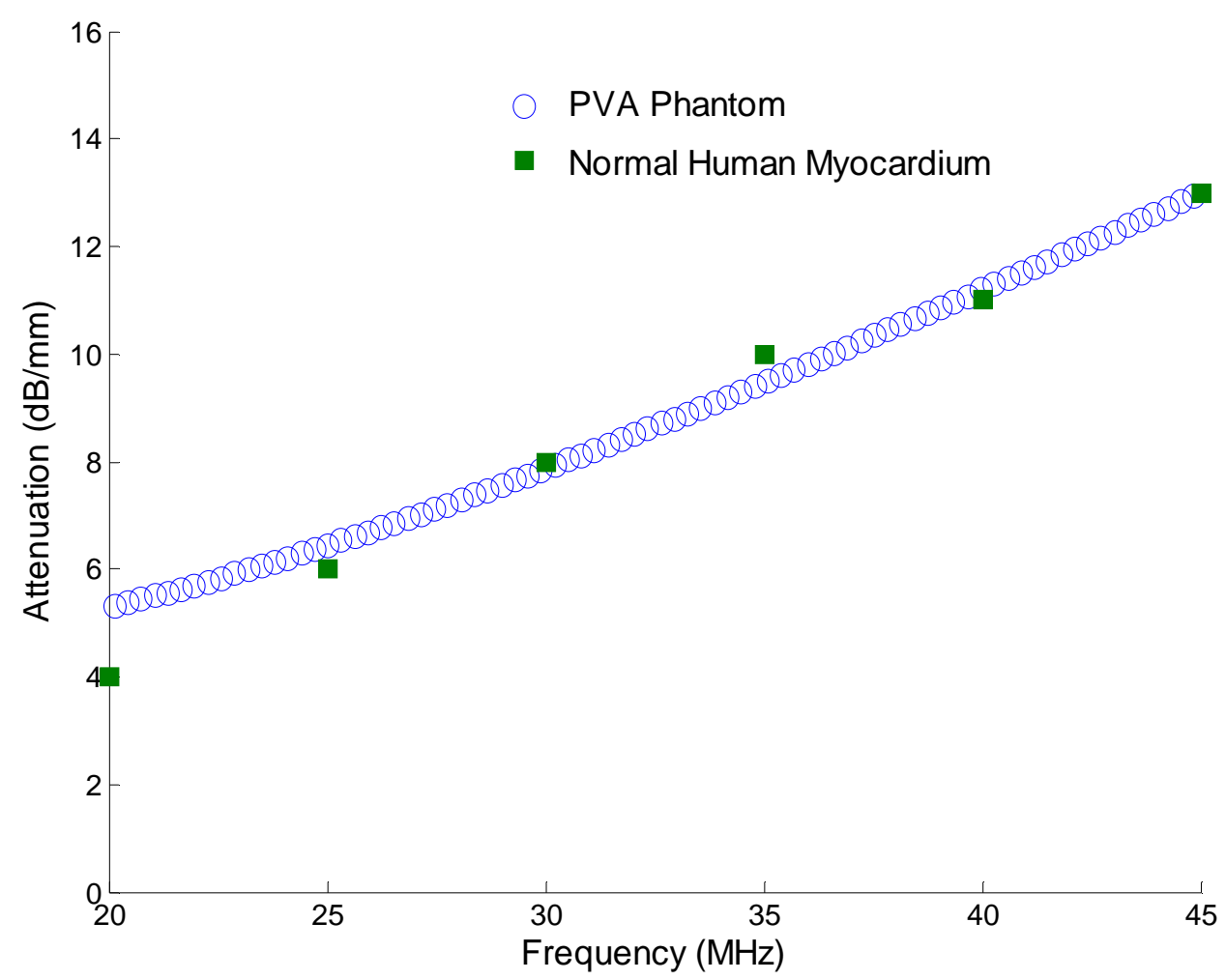

Figure 7.9 - Attenuation coefficient versus frequency for $7 \%$ concentration by weight PVA phantom (circles) measured along the direction of PVA alignment (freeze casting direction). Also plotted are measurements made parallel to the fibre alignment on normal human myocardium tissue (filled squares) obtained from [127].

Whilst the above phantom shows attenuation properties representative of human myocardium tissue, like velocity the PVA attenuation properties are not limited to the above. Varying the process parameters can realise phantoms with attenuation or velocity properties significantly greater or less than the above example phantom, and these results should therefore be viewed as representative of what can be attained. The samples can also be scaled and made any size provided the same procedure is followed and rigorous control applied during the sample immersion step. 


\section{Chapter 8}

\section{Conclusion \& Discussion}

The discussion of the results from this work are summarised in three main sections of this chapter: Section 8.1 summarises the finite element modelling (FEM) of high frequency wave propagation in collinear porous microstructures from Chapter 5, section 8.2 the experimental characterisation of the in-house fabricated porous anodic alumina samples (PAA) analysed in Chapter 6, and section 8.3 the fabrication and characterisation of the novel microstructured poly(vinyl alcohol) tissue mimicking phantoms described in Chapter 7.

\subsection{Finite Element Modelling of High Frequency Ultrasonic Wave Propagation in Microstructured Materials}

FEM performed using PZFlex revealed highly regular microstructures significantly influence high frequency (20-200 MHz) ultrasonic wave propagation, with a highly collinear microstructure having a major waveguiding affect. The dimensions and collinear structure of these samples constrain the propagating waves causing them to travel as a compact waveform through the layer with no scattering.

The 2D modelling of a collinear porous aluminium material immersed in water revealed the influence microstructure has on high frequency waves, with wavelengths comparable to or 
larger than the porous layer solid dimensions. For a porous aluminium layer with $4 \mu \mathrm{m}$ sized pillars separated by water filled channels of the same width, it was observed for a $50 \mathrm{MHz}$ wave (with a wavelength of approximately $30 \mu \mathrm{m}$ ) excited on a single pillar, the wave was strongly guided along its length. The waveform was constrained to the pillar, with very little leakage through the water to adjacent pillars. The wave travelled as a single wavefront in the excited pillar at a guided velocity of $5400 \mathrm{~ms}^{-1}$. A slow wave generated by the wake of the primary wave was observed in the water travelling at the $\mathrm{P}$ wave velocity of water of $1480 \mathrm{~ms}^{-1}$. This is in contrast to that observed for solid aluminium where both $\mathrm{P}$ and $\mathrm{S}$ waves were observed. In the homogeneous bulk material, these waves are unconstrained and are free to spread circularly outwards at their own respective velocities of $6300 \mathrm{~ms}^{-1}$ and $3100 \mathrm{~ms}^{-1}$. The slow wave observed for both the single pillar and plane wave excitations contained significantly less energy than the fast wave unless microstructured layers with porosities of $90 \%$ or greater were used.

For a spherical source generated in water propagating into the same $2 \mathrm{D}$ porous aluminium layer, the normalising capabilities of such a microstructure were observed. The wavefront from the spherical source, containing all angles of incidence, upon reaching the interface of the porous layer displayed no critical angles, with transmission into the layer occurring for all angles of incidence. Within the porous layer the wave shape was preserved. Waveshape preservation is of value since this reveals information about the nature of the scattering source, important for imaging applications. Since the porous layer is acting as a waveguide to the incident waves, the transmission and reflection phenomena that occur for homogeneous media are no longer observed. Hence the wave propagation is not restricted by the $\mathrm{P}$ and $\mathrm{S}$ critical angles that exist for bulk solids.

At the interface between the porous aluminium layer and aluminium solid, a reflected wave was observed to propagate back into the porous layer. Given the physical material properties (density, longitudinal velocity), and thus characteristic impedance of the individual aluminium pillars of the porous layer and solid aluminium were the same, an acoustic impedance mismatch based on material properties does not exist. However, the different wave propagation characteristics of the guided wave in the plate and bulk waves in the solid cause this mismatch. 
This phenomena was further investigated by simulating a free boundary aluminium plate that widened into a homogenous aluminium solid. By varying the thickness of the plate, the size of the reflected pulse could be varied and thus the impedance mismatch. By widening the plate the situation becomes closer to that of a continuous homogeneous solid, resulting in a smaller reflected pulse due to a smaller acoustic impedance mismatch. Extending this concept to the porous aluminium layer, by controlling the layer porosity the acoustic impedance of the sample can be varied to a desired value.

Taking into account the 2D modelling results, 3D models were then performed to provide a realistic representation of the fabricated PAA samples. These results could be compared with those measured from experimental characterisation of the PAA samples. These simulations found the PAA sample acoustic impedance to be independent of both sample architecture and dimensions, but displayed a linear dependence on the sample porosity. By varying the porosity over the range 0 to $80 \%$, sample impedances in the range of 2-17 MRayl could be achieved. The acoustic impedance was found to be independent of frequency to at least $200 \mathrm{MHz}$.

The PAA sample velocity was found to be dispersionless to at least $200 \mathrm{MHz}$. It was highly dependent on the sample porosity and unlike the acoustic impedance did show a small dependence on the sample architecture. For low porosities the velocity was found to be independent of architecture, with velocity decreasing from the $\mathrm{P}$ wave velocity, in this case aluminium with a velocity of $6300 \mathrm{~ms}^{-1}$. This continued until approximately $13 \%$ porosity, where a velocity of $5900 \mathrm{~ms}^{-1}$ was observed, at which point the velocities of the two architectures began to separate. For the triangular model, at high porosity the velocity appeared to approach the fundamental bar velocity of $5090 \mathrm{~ms}^{-1}$, whilst for the rectangular model the velocity approaches the fundamental symmetric Lamb mode velocity of $5400 \mathrm{~ms}^{-1}$.

In future work the regularity of these highly collinear structures will be reduced. This will allow for the understanding of wave propagation in more complex structures, with less collinearity, but still highly anisotropic. This heads towards the modelling of real tissue like structures, revealing the wave guiding phenomena associated with these. By simulating these structures with the same acoustic properties as tissue the results from these simulations can be compared with experimental measurements performed on the anisotropic PVA phantoms developed in this work. 


\subsection{Experimental Characterisation of Porous Anodic Alumina Samples for Use As Acoustic Matching Layers}

Experimental velocity measurements performed on the in-house fabricated PAA samples revealed guided wave propagation, with velocity dependent upon sample porosity. Measured values were in good agreement with those calculated from the modelling at the corresponding porosities. Experimental velocity measurements were performed to $175 \mathrm{MHz}$ displaying no frequency dependence, confirming the FEM modelling. Only a single waveform was received corresponding to the fast wave identified in the modelling. The slow wave was not observed due to the small energy this wave contained.

The normalising characteristics of the PAA samples identified in the modelling were confirmed experimentally. Velocities were measured for angles of incidence out past $40^{\circ}$, well beyond the $\mathrm{P}$ and S critical angles measured for solid aluminium of $14^{\circ}$ and $29^{\circ}$ respectively. Similar behaviour was observed for the commercial PAA samples where energy was received for angles greater than the aluminium $\mathrm{P}$ and $\mathrm{S}$ critical angles, however the amplitudes were significantly less than those measured for the in-house fabricated PAA samples. The velocities measured across the commercial samples for a range of angles were found to be highly variable, in significant contrast to the constant velocities measured for the in-house fabricated PAA samples. This suggested significant sample path dependence, a likely consequence of the poor regularity of the commercial samples. Using a highly collinear microstructured layer allows for the reception of energy over a much wider range of angles, increasing the signal to noise ratio, and allowing the transmission of signals that would potentially go critical at a solid media interface, and thus be undetected.

Experimental acoustic impedance measurements of the three types of PAA samples were in good agreement with those in the modelling at the respective sample porosities. The lowest acoustic impedance measured experimentally of 9 MRayl was achieved for the most porous type 3 samples with porosities in the range of 45-50\%. These samples were still very robust at these porosities and by fabricating samples with porosities as high as $70 \%$, impedances as low as 4 MRayl can potentially be achieved. 
The additional property not simulated but measured experimentally was the attenuation of the PAA samples. The measured attenuation characteristics of the samples were orders of magnitude less than those found in the literature for the currently used epoxy powder matching layers. At the highest frequency for which the coefficients were measured (175 MHz), the smallest attenuation coefficient of the PAA samples was just $8 \mathrm{dBmm}^{-1}$, and the largest still only $22 \mathrm{dBmm}^{-1}$. With a quoted attenuation coefficient for the epoxy/powder mixtures of $500 \mathrm{dBmm}^{-1}$ at $100 \mathrm{MHz}$, compared with the highest value measured at $100 \mathrm{MHz}$ for the type 3 PAA samples of no more than $15 \mathrm{dBmm}^{-1}$, a significant difference in attenuation performance can be concluded. This is an enormous reduction in attenuation, and at frequencies where the reduction of attenuation is critical to achieving a meaningful signal.

With no measurable dispersive velocity characteristics, tailorable impedances potentially covering a wide range of useful impedances, very low attenuation coefficients, and reception over a wide range of angles, these nanomaterials are an excellent potential future alternative for transducer matching layers. In future work, samples with fabricated porosities up to 60-70\% will be attempted to realise acoustic impedances in the range of 4-6 MRayl. The influence of filling the pores with other couplants and solids is also of interest, as these will also influence the final material properties. The anodisation of other materials is also under consideration to achieve alternative impedance ranges.

\subsection{Microstructured Poly(Vinyl Alcohol) Tissue Mimicking Phantoms}

Anisotropic PVA tissue mimicking phantoms were prepared using a novel freeze casting process. PVA tissue phantoms currently used by ultrasound researchers possess similar bulk acoustic properties such as velocity and acoustic impedance to those of real tissues. These phantoms however are homogeneous and fail to incorporate the microstructure and dimensions found in real fibrous tissues. With ultrasound imaging systems continuing towards $100 \mathrm{MHz}$ and beyond, unless the microstructure dimensions of the underlying tissue substructure are considered, the imaging quality of the system will be degraded.

With the lack of a suitable phantom to carry out this work, the freeze casting method used for fabricating advanced monolithic ceramics was modified in order to create a microstructured 
tissue phantom. The freeze casting process is very simple, with the properties of the final phantom largely determined by the size of the ice crystals that form in the slurry during the immersion process. The size of the ice crystals is mainly dependant upon the rate of lowering of the sample into the liquid nitrogen, and the concentration of the PVA slurry used.

These samples possess the acoustic properties (velocity, attenuation) found in current tissue phantoms, but also contain the same microstructural properties (anisotropy, local variations, microstructure dimensions) similar to those found in real fibrous tissues. By varying the freezing rate, PVA slurry concentration, and PVA molecular weight, phantoms with different properties can be fabricated.

Horizontal and vertical cross sections of example PVA phantoms examined using SEM reveals a porous PVA microstructure where the ice crystals had been removed by freeze-drying. The pore walls were $10-20 \mu \mathrm{m}$ thick with pore sizes of approximately $20-100 \mu \mathrm{m}$. A preferred orientation was also observed that formed along the direction of freeze casting. Experimental measurements of the acoustic properties of these phantoms over the frequency range 20-45 $\mathrm{MHz}$ reveal velocity and attenuation coefficients in good agreement with those measured in the literature. Velocity measurements that were made parallel to the direction of PVA alignment were greater than those made perpendicular (as found in real tissues). The magnitude and rate of change of these velocities with frequency were also in good agreement with real tissue over the same frequency range. Attenuation measurements of an example PVA phantom was in excellent agreement with measurements performed on healthy human heart tissue (myocardium) over the same frequency range.

Using a simple freeze casting fabrication process, phantoms possessing measured acoustic and microstructural properties representative of real fibrous tissues have been fabricated. These phantoms are a significant step forward in the fabrication of more advanced realistic tissue phantoms for high frequency tissue characterisation studies. To extend upon this initial freeze casting process presented, the influence of introducing pre-nucleation templates will be explored in planned future work. Fabricating a regular microfabricated template containing regularly spaced square wells on the base of the sample holder will force ice crystals to form to the microstructure dimensions. By changing the dimensions of this seed layer, a greater control 
over the range of ice crystal sizes in a phantom can be obtained. This will allow for a greater range of pore wall thicknesses and increase the regularity of the vertically formed ice pillars.

Both regular and irregular nucleation templates will be introduced onto the base of the freezecasting container. Filling the samples with other materials is also of interest to create composite tissue phantoms with specific properties. Efforts to reduce the solubility of the samples will also be made. Repeated cryogenic freezing/thawing cycles similar to the procedure followed for the homogeneous phantoms could also be applied to these phantoms. The cryogenic temperature gradient will cause the preferred orientation within the PVA during each cycle, whilst the repeated cycles will cause more of the PVA molecules to form stronger bonds, improving the alignment of the structure with each cycle. 


\section{References}

[1] K. R. Erikson, F. J. Fry, J. P. Jones, "Ultrasound in Medicine - A Review," IEEE Transactions on Sonics and Ultrasonics, Vol. SU-21, 1974, pp. 144-170.

[2] S. Foster, K. A. Harasiewicz, M. D. Sherar, "History of Medical and Biological Imaging with Polyvinylidene Fluoride (PVDF) Transducers,“ IEEE Transactions on Ultrasonics, Ferroelectrics, and Frequency Control, Vol. 47, 2000, pp. 1363-1371.

[3] G. R. Harris, "Medical Ultrasound Exposure Measurements: Update on Devices, Methods, and Problems," Proceedings of the IEEE International Ultrasonics Symposium, Lake Tahoe, 1999, pp. 13411352.

[4] M.Vogt, S.Scharenberg, R.Scharenberg, H.Ermert, "In vivo Ultrasound Biomicroscopy of Skin with $20 \mathrm{MHz}$ and $100 \mathrm{MHz}$ Range Ultrasound: Inverse Echo Signal Filtering Optimization,” Proceedings of the IEEE International Ultrasonics Symposium, Rotterdam, 2005.

[5] L. Josa, P. Kannus, "Human Tendon: Anatomy, Physiology, and Pathology," Champaign, Human Kinetics, 1997.

[6] T. Yasui, Y. Tohno, T. Araki, "Characterisation of Collagen Orientation in Human Dermis by TwoDimensional Second-Harmonic-Generation Polarimetry," Journal of Biomedical Optics, Vol 9, 2004, p 259.

[7] L. C. Junqueira, G. S. Montes, J. E. Martins, P. P. Joazeiro, “Dermal Collagen Distribution,” Journal of Histochemistry and Cell Biology, Vol. 79, 1983, pp. 397-403.

[8] H.Kafantari, E.Kounadi, M.Fatouros, M.Milonakis, M.Tzaphlidou, "Structural Alterations in Rat Skin and Bone Collagen Fibrils Induced by Ovariectomy,” Bone, Vol, 2000, pp. 349-353.

[9] G.Fountos et al, "The Effects of Inflammation Mediated Osteoporosis on the Skeletal Ca/P Ratio and on the Structure of Rabbit Bone and Skin Collagen," Journal of Applied Radiation and Isotopes, Vol 49, 1998, pp. 657-659.

[10] M. Savvas et al, "The Effect of Anorexia Nervosa on Skin Thickness, Skin Collagen and Bone Density," Journal of Obstetrics and Gynaecology, Vol 96, 1989, pp. 1392-1394.

[11] C.Fournier, S.Bridal, A.Coron, P.Laugier, "Relationship Between Skin Collagen Microartitecture and Ultrasonic Backscatter Parameters at 20MHz," Proccedings of IEEE International Ultrasonics Symposium, Munich, 2002.

[12] W. Smith, "Composite Piezoelectric Materials for Medical Ultrasonic Imaging Transducers - A Review," Proceedings of IEEE International Ultrasonics Symposium, 1986, pp. 212-223.

[13] Y. Ito, K. Kushida, K. Sugawara, H. Takeuchi, “A 100-MHz Ultrasonic Transducer Array Using ZnO Thin Films," IEEE Transactions on Ultrasonics, Ferroelectrics, and Frequency Control, Vol. 42, 1995, pp. 316-324.

[14] S. Foster, K. A. Harasiewicz, M. Sherar, "A History of Medical and Biological Imaging with Polyvinylidene Fluoride (PVDF) Transducers," IEEE Transactions on Ultrasonics, Ferroelectrics, and Frequency Control, Vol. 47, 2000, pp. 1363-1371. 
[15] A. Shoh, "Industrial Applications of Ultrasound - A Review I. High-Power Ultrasound," IEEE Transactions on Ultrasonics, Ferroelectrics, and Frequency Control, Vol. 22, 1975, pp. 60-71.

[16] L. C. Lynnworth, "Industrial Applications of Ultrasound - A Review II. Measurements, Tests, and Process Control Uing Low-Intensity Ultrasound," IEEE Transactions on Ultrasonics, Ferroelectrics, and Frequency Control, Vol. 22, 1975, pp. 71-101.

[17] RIMA International, “Oxidation Of Aluminum Foil FacingsUsed in Reflective Insulation, " Technical Bulletin No. 105, March, 2008.

[18] L. W. Braile "Seismic Waves and the Slinky," web.ics.purdue.edu/ braile/edumod/slinky/slinky.htm.

[19] J. L. Rose, "Ultrasonic Waves in Solid Media," Cambridge, Cambridge University Press, 2004.

[20] K. F. Graff, “Wave Motion in Elastic Solids,” New York, Dover Publications, 1991.

[21] H. Lamb, “On Waves in an Elastic Plate," Proceedings of the Royal Society, Serial A, 93, 114, 1917.

[22] D. Royer, E. Dieulesaint, "Elastic Waves in Solids," Berlin, Springer-Verlag, 1999.

[23] I. A. Viktorov, "Rayleigh and Lamb Waves - Physical Theory and Applications," New York, Plenum Press, 1967.

[24] J. D. Cheeke, "Fundamentals and Applications of Ultrasonic Waves," Boca Raton, CRC Press, 2002.

[25] W. H. Press, "Numerical Recipes in Pascal," Cambridge, Cambridge University Press, 1989.

[26] M. F. Osborne, S. D. Hart, "Transmission, Reflection, and Guiding of an Exponential Pulse by a Steel Plate in Water. I. Theory," Journal of the Acoustical Society of America, Vol. 17, 1945, pp. 1-18.

[27] M. F. Osborne, S. D. Hart, "Transmission, Reflection, and Guiding of an Exponential Pulse by a Steel Plate in Water. II. Experimental,” Journal of the Acoustical Society of America, Vol. 18, 1946, pp. 251252.

[28] X. L. Bao, H. Franklin, P. K.Raju, H. Uberall, "The Splitting of Dispersion Curves for Plates FluidLoaded on Both Sides," Journal of the Acoustical Society of America, Vol. 102, 1997, pp. 1246.

[29] J. Miklowitz, “The Theory of Elastic Waves and Waveguides,” New York, North-Holland, 1978.

[30] J. D. Achenbach, "Wave Propagation in Elastic Solids,” New York, North-Holland, 1984.

[31] J. W. Rayleigh, “On Waves Propagating Along the Plane Surface of an Elastic Solid,” Proceedings of the London Mathematical Society, Vol. 17, 4, 1885.

[32] P. Chadwick, G. D. Smith, "Foundations of the Theory of Surface Waves in Anisotropic Elastic Materials," Advanced Applied Mechanics, Vol. 17, 1977, pp. 303-377.

[33] L. E. Kinsler, A. R. Frey, A. B. Coppens, J. V. Sanders, "Fundamentals of Acoustics," 4th edition, New Jersey, Wiley, 1999.

[34] G. Kossoff, "The Effects of Backing and Matching on the Performance of Piezoelectric Ceramic Transducers," IEEE Transactions on Sonics and Ultrasonics, Vol. SU-13, pp. 20-30, 1966.

[35] J. H. Goil, B. A. Auld, "Multilayer Impedance Matching Schemes for Broadbanding of Water Loaded Piezoelectric Transducers and High Q Electric Resonators," IEEE Transactions on Sonics and Ultrasonics, Vol. SU-22, pp. 52-53, 1975.

[36] C. S. Desilets, J. D. Fraser, G. S. Kino, "The Design of Efficient Broad-band Piezoelectric Transducers," IEEE Transactions on Sonics and Ultrasonics, SU-25, pp. 115-125, 1978. 
[37] S. Thiagarajan, R. W. Martin, A. Proctor, I. Jayawadena, F. Silverstein, "Dual Layer Matching (20 MHz) Piezoelectric Transducers with Glass and Parylene," IEEE Transactions on Ultrasonics, Ferroelectrics, and Frequency Control, Vol. 44, pp. 1172-1174, 1997.

[38] J. H. Goll, “The Design of Broad-band Fluid Loaded Ultrasonic Transducers," IEEE Transactions on Sonics and Ultrasonics, Vol. SU-26, pp. 75-81, 1979.

[39] T. Inoue, M. Ohta, S. Takahashi, "Design of Ultrasonic Transducers with Multiple Acoustic Matching Layers for Medical Applications," IEEE Transactions on Ultrasonics, Ferroelectrics, and Frequency Control, Vol. 34, pp. 8-16, 1987.

[40] F. A. Duck, "Physical Properties of Tissue," 1st edition, New York, Elsevier Academic Press, 1990.

[41] D. E. Chimenti, A. H. Nayfeh, "Ultrasonic Reflection and Guided Wave Propagation in Biaxially Laminated Composite Plates," Journal of the Acoustical Society of America," Vol. 87, pp. 1409-1415, 1990.

[42] D. E. Chimenti, "Guided Waves in Plates and their use in Materials Characterisation," Journal of Applied Mechanics,” Vol. 50, pp. 247-284, 1997.

[43] A. J. Niklasson, S. K. Datta, M. L. Dunn, "On Ultrasonic Guided Waves in a Thin Anisotropic Layer Lying Between Two Isotropic Layers," Journal of the Acoustical Society of America," Vol. 108, pp. 2005-2011, 2000.

[44] A. H. Nayfeh, "Wave Propagation in Layered Anisotropic Media with Applications to Composites," Amsterdam, North Holland, 2000.

[45] H. Zhang, S. Fan, Z. Liu, D. Lu, D. Ta, "The Effect of Fibre Orientation on Lamb Wave Propagation in Layered Anisotropic Composite Plates," Proceedings of IEEE International Ultrasonics Symposium, Vancouver, pp. 1821-1823, 2006.

[46] V. Pagneux, A. Maurel, "Lamb Wave Propagation in Inhomogeneous Elastic Waveguides," Proceedings of the Royal Society of London, Vol. A, pp. 1913-1930, 2002.

[47] Q. Fan, J. Takatsubo, S. Yamamoto, "Quantitative Characterisation of Advanced Porous Ceramics Based on a Probabilistic Theory of Ultrasonic Wave Propagation,” Journal of Applied Physics, Vol. 86, pp. 4023-4028, 1999.

[48] A. Huczko, Journal of Applied Physics, Vol. A70, p. 365, 2000.

[49] A. P. Li, F. Miller, A. Birner, K. Nielsch, U. Gosele, Journal of Advanced Materials, Vol. 11, p. 483, 1999.

[50] M. Saito, M. Kirihara, T. Taniguchi, M. Miyagi, Journal of Applied Physics, p. 607, 1989.

[51] S. De Vito, C. R. Martin, Chemistry Materials, p. Vol. 10, p. 1738, 1998.

[52] B. Z. Tang, H. Xu, Macromolecules, Vol. 32, p. 2569, 1999.

[53] C. R. Martin, Chemistry Materials, Vol. 8, p. 1739, 1996.

[54] A. J. Kinloch, "Durability of Structural Adhesives," Applied Science, London, 1983.

[55] T. Sano, N. Iguchi, K. Iida, T. Sakamoto, M. Baba, H. Kawaura, "Size-exclusion chromatography using Self-organized nanopores in anodic porous aluminium," Journal of Applied Physics Letters, Vol. 83, p. 4438, 2003. 
[56] H. L. Lira, R. Patterson, "New and Modified Anodic Alumina Membranes - Part III. Preparation and Characterisation by Gas Diffusion of $5 \mathrm{~nm}$ Pore Size Anodic Alumina Membranes, Journal of Membrane Science, Vol. 206, p. 375, 2002.

[57] Z. Sklar, G. A. Briggs, P. Cawley, A. J. Kinloch, "Quantitative Acoustic Microscopy of Anodized and Coated Aluminium at Frequencies up to 1 GHz," Journal of Materials Science, Vol. 30, pp. 3752-3760, 1995.

[58] P. Zinin, O. Lefeuvre, A. Briggs, B. D. Zeller, P. Cawley, A. Kinloch, X. Zhou, G. Thompson, "Determination of Density and Elastic Constants of a Thin Phosphoric Acid Anodized Oxide Film by Acoustic Microscopy," Journal of the Acoustical Society of America, Vol. 106, pp. 2560-2567, 1999.

[59] M. Asmani, C. Kermel, A. Leriche, M. Ourak, "Influence of Porosity on Young's Modulus and Poisson's Ratio in Alumina Ceramics," Journal of the European Ceramic Society, Vol. 21, pp. 10811086, 2000.

[60] Z. Xia et al., "Mechanical Properties of Highly Ordered Nanoporous Anodic Alumina Membranes," Journal of Advanced Materials Science, Vol. 6, pp. 131-139, 2004.

[61] S. H. Ko et al., "Mechanical Properties and Residual Stress Measurements in Anodic Aluminium Oxide Structures Using Nanoindentation," Proceedings of Meeting on Nanoparticles, Nanostructures, and Nanocomposites, St. Petersburg, pp. 356-363, 2004.

[62] D. J. Green, C. Nader, R. Brezny, "The Elastic Behaviour of Partially Sintered Alumina," The Ceramic Transactions, pp. 345-356, 1990.

[63] A. Kirchner, K. J. Mackenzie, I. W. Brown, T. Kemmit, M. E. Bowden, "Structural Characterisation of Heat-Treated Anodic Alumina Membranes Prepared Using a Simplified Fabrication Process," Journal of Membrane Science, 287, 2007, pp. 264-270.

[64] N. Akashi, J. Kushibiki, N. Chubachi, "Acoustic Properties of Selected Bovine Tissues in the Frequency Range 20-200 MHz,” Journal of the Acoustical Society of America, Vol. 98, 1995, pp. 3035-3039.

[65] C. Fournier, S. L. Bridal, A. Coron, P. Laugier, "Relationship between Skin-collagen Micro-architecture and Ultrasonic Backscatter Parameters at $20 \mathrm{MHz}$," Proceedings of the IEEE International Ultrasonics Symposium, Munich, pp. 625-628, 2002.

[66] B. I. Raju, K. J. Swindells, S. Gonzalez, M. A. Srinivasan, "Quantitative Ultrasonic Methods for Characterisation of Skin Lesions In Vivo," Journal of Ultrasound in Medicine and Biology, Vol. 29, pp. 825-838, 2003.

[67] T. L. Szabo, "Diagnostic Ultrasound Imaging: Inside Out," San Diego, Elsevier Academic Press, 2004.

[68] G. S. Kino, “Acoustic Waves: Devices, Imaging, and Analog Signal Processing,” Englewood Cliffs, Prentice Hall, 1987, pp. 300-357.

[69] D. E. Goertz, S. W. Wong, C. T. Chin, E. Cherin, P. N. Burns, F. S. Foster, "Non-linear Scattering from Microbubble Contrast Agents in the 14-40 MHz Range," Proceedings of IEEE International Ultrasonics Symposium, 2001, Vol. 2, pp. 1747-1750.

[70] P. M. Morse, K. U. Ingard, “Theoretical Acoustics,” Princeton, Princeton University Press, 1968, pp. 400-411.

[71] J. W. Strutt (Lord Rayleigh), “On the Light From the Sky, its Polarization and Colour," Scientific Papers Lord Rayleigh, Vol. 1, New York, Dover Publications, 1964 (reprint of 1871). 
[72] J. J. Faran, "Sound Scattering by Solid Cylinders and Spheres," Journal of the Acoustical Society of America, Vol. 23, 1951, pp. 405-418.

[73] R. Hickling, “Analysis of Echoes From Solid Elastic Sphere in Water," Journal of the Acoustical Society of America, Vol. 34, 1962, pp. 1582-1592.

[74] D. K. Nassiri, C. R. Hill, "The Use of Angular Acoustic Scattering Measurements to Estimate Structural Parameters of Human and Animal Tissues," Journal of the Acoustical Society of America, Vol. 79, 1986, pp. 2048-2054.

[75] R. B. Lindsay, “Mechanical Radiation,” New York, McGraw Hill, 1960.

[76] NDT Resource Centre, www.ndt-ed.org/index_flash.htm.

[77] Olympus transducers technical notes, www.olympus-ims.com/en/panametrics-ndt-ultrasonic/pdf.

[78] D. Hsu, M. Hughes, "Simultaneous Ultrasonic Velocity and Sample Thickness Measurement and Application in Composites," Journal of the Acoustical Society of America, 1992, pp. 669-675.

[79] H. Wang, W. Cao, "Improved Ultrasonic Spectroscopy Methods for Characterisation of Dispersive Materials," IEEE Transactions on Ultrasonics, Ferroelectrics, and Frequency Control, Vol. 48, 2001, pp. 1060-1065.

[80] P. He, "Measurement of Acoustic Dispersion using both Transmitted and Reflected Pulses," Journal of the Acoustical Society of America, Vol. 107, 1999, pp. 801-807.

[81] H. Wang, W. Jiang, W. Cao, "Characterisation of Lead Zirconate Titanate Piezoceramic Using High frequency Ultrasonic Spectroscopy,” Journal of Applied Physics, Vol. 85, 1999, pp. 8083-8091.

[82] V. A. Del Grosso, C. W. Mades, "Speed of Sound in Pure Water," Journal of the Acoustical Society of America, Vol. 52, 1972, pp. 1442-1446.

[83] W. Marczak, "Water as a Standard in the Measurements of Speed of Sound in Liquids," Journal of the Acoustical Society of America”, Vol. 102, 1997, pp. 2776-2779.

[84] T. R. Gururaja, W. A. Schulze, L. E. Cross, R. E. Newnham, "Piezoelectric Composite Materials for Ultrasonic Transducer Applications. Part II: Evaluation of Ultrasonic Medical Applications," IEEE Transactions on Sonics and Ultrasonics, Vol. 32, 1985, pp. 499-512.

[85] Olympus, www.olympus-ims.com/en/panametrics-ndt-ultrasonic/highfrequency.

[86] Agilent Technologies, “Agilent 4291B RF Impedance/Material Analyser Operation Manual”, 3rd edition, Japan, 1999.

[87] A. S. Sedra, K. C. Smith, "Microelectronic Circuits," 5th edition, Oxford, Oxford University Press, 2004.

[88] J. G. Proakis, "Digital Signal Processing: Principles, Algorithms, and Applications," 3rd Edition, Prentice Hall, 1995.

[89] S. W. Smith, “The Scientists and Engineers's Guide to Digital Signal Processing," San Diego, California Technical Publishing, 1997.

[90] J. P. O’Sullivan, G. C. Wood, “The Morphology and Mechanism of Formation of Porous Anodic Films on Aluminium," Proceedings of the Royal Society of London A, Vol. 317, 1970, p. 511.

[91] R. C. Ferneaux, W. R. Rigby, A. P. Davidson, “The Formation of Controlled Porosity Membranes from Anodically Oxidised Aluminium, “ Nature, Vol. 337, 1989, p. 147. 
[92] H. Masuda, F. Hasegawa, S. Ono, "Self-ordering of Cell Configuration of Anodic Porous Alumina Formed in Sulphuric Acid Solution,” Journal of Electrochemical Society, Vol. 144, 1997, p. 127.

[93] H. Masuda, K. Yada, A. Osaka, "Self-Ordering of Cell Configuration of Anodic Porous Alumina with Large-Size Pores in Phosphoric Acid Solutions," Japanese Journal of Applied Physics, Vol. 37, 1998, p. 1340.

[94] W. Lee, R. Ji, U. Gosele, K. Nielsch, "Fast Fabrication of Long-Range Ordered Porous Alumina Membranes by Hard Anodization,“ Nature Materials, Vol. 5, 2006, pp. 741-747.

[95] A. P. Li, F. Muller, A. Birner, K. Nielsch, U. Gosele, "Hexagonal Pore Arrays with a 50-420 nm Interpore Distance Formed by Self-Organisation in Anodic Alumina,“ Journal of Applied Physics, Vol. 84, 1998, pp. 6023-6026.

[96] M. J. Zipparo, K. K. Shung, T. R. Shrout, "Piezoceramics for High-Frequency (20 to $100 \mathrm{MHz}$ ) SingleElement Imaging Transducers," IEEE Transactions on Ultrasonics, Ferroelectrics, and Frequency Control, Vol. 44, 1997, pp. 1038-1047.

[97] K. A. Snook, J. Zhao, C. H. Alves, J. M. Cannata, W. Chen, R. J. Meyer, T. A. Ritter, K. K. Shung, "Design, Fabrication, and Evaluation of High Frequency, Single-Element Transducers Incorporating Different Materials," IEEE Transactions on Ultrasonics, Ferroelectrics, and Frequency Control, Vol. 49, 2002, pp. 169-176.

[98] H. Wang, T. Ritter, W. Cao, K. K. Shung, "High Frequency Properties of Passive materials for Ultrasonic Transducers," IEEE Transactions on Ultrasonics, Ferroelectrics, and Frequency Control, Vol. 48, 2001, pp. 78-84.

[99] H. Guo, J. M. Cannata, Q. Zhou, K. K. Shung, "Design and Fabrication of Broadband Graded Ultrasonic Transducers with Rectangular Kerfs," IEEE Transactions on Ultrasonics, Ferroelectrics, and Frequency Control, Vol. 52, 2005, pp. 2096-2102.

[100] Q. Zhou, J. M. Cannata, H. Guo, C. Huang, V. Z. Marmarelis, K. K. Shung, "Half-Thickness Inversion Layer High-Frequency Ultrasonic Transducers Using $\mathrm{LiNbO}_{3}$ Single Crystal," IEEE Transactions on Ultrasonics, Ferroelectrics, and Frequency Control, Vol. 52, 2005, pp. 127-133.

[101] R. Zhang, W. Cao, Q. Zhou, J. H. Cha, K. K. Shung, Y. Huang, "Acoustic Properties of Alumina Colloidal/Polymer Nano-Composite Film on Silicon," IEEE Transactions on Ultrasonics, Ferroelectrics, and Frequency Control, Vol. 54, 2007, pp. 467-469.

[102] Q. Zhou, J. H. Cha, Y. Huang, R. Zhang, W. Cao, K. K. Shung, "Alumina/Epoxy Nanocomposite Matching Layers for High-Frequency Ultrasound Transducer Application," IEEE Transactions on Ultrasonics, Ferroelectrics, and Frequency Control, Vol. 56, 2009, pp. 213-219.

[103] F. Tiefensee, C. Becker-Willinger, G. Heppe, P. Herbeck-Engel, A. Jakob, "Nanocomposite Cerium Oxide Polymer Matching Layers with Adjustable Acoustic Impedance Between 4 MRayl and 7 MRayl," Ultrasonics, Elsevier, Sept, 2009, in press.

[104] T. E. Gomez, F. Montero, "Bridging the Gap of Impedance Mismatch Between Air and Solid Materials," Proceedings of the IEEE International Ultrasonics Symposium, 2000.

[105] T. E. Gomez, "Acoustic Impedance Matching of Piezoelectric Transducers to the Air," IEEE Transactions on Ultrasonics, Ferroelectrics, and Frequency Control, Vol. 51, 2009, pp. 624-633.

[106] www.whatman.com/products. 
[107] PZFlex, Weidlinger Associates, www.pzflex.com.

[108] G. Alcala, P. Skeldon, G. E. Thompson, A. B. Mann, H. Habazaki, K. Shimizu, "Mechanical Properties of Amorphous Anodic Alumina and Tantala Films Using Nanoindentation," Nanotechnology, Vol. 13, pp. 451-455, 2002.

[109] Y. Kijima, T. Hanada, "Effect of the Pressure of Sputtering Atmosphere on the Physical Properties of Amorphous Aluminium Oxide3 Films,” Journal of Materials Science, Vol. 35, pp. 2193-2199, 2000.

[110] G. T. Mearini, R. W. Hoffman, "Tensile Properties of Aluminium/Alumina Multi-Layered Thin Films," Journal of Electronic Materials, Vol. 22, pp. 623-629, 1993.

[111] PZFlex, Weidlinger Associates, "PZFlex command reference manual," www.pzflex.com.

[112] L. B. Jackson, “Digital Filters and Signal Processing,” Boston, MA: Kluwer, 1986, pp. 85-92.

[113] N. A. Peppas, S. R. Stauffer, "Reinforced Uncrosslinked Poly(Vinyl Alcohol) Gels Produced by CyclicFreezing-Thawing Processes: A Short Review," Journal of Controlled Release, Vol. 16, 1991, pp. 305310 .

[114] J. Fromageau, E. Brusseau, D. Vray, G. Gimenez, P. Delachartre, "Characterisation of PVA Cryogel for Intravascular Ultrasound Elasticity Imaging," IEEE Transactions on Ultrasonics, Ferroelectrics, and Frequency Control, Vol. 50, 2003, pp. 1318-1323.

[115] W. Khaled, T. Neumann, H. Ermert, S. Reichling, A. Arnold, O. T. Bruhns, "Evaluation of Material Parameters of PVA Phantoms for Reconstructive Ultrasound Elastography," Proceedings of the IEEE International Ultrasonics Symposium, 2007, pp. 1329-1332.

[116] J. Fromageau, J. Gennisson, C. Schmitt, R. Maurice, R. Mongrain, G. Cloutier, "Estimation of Poly(Vinyl Alcohol) Cryogel Mechanical Properties with Four Ultrasound Elastography Methods and Comparison with Gold Standard Testings," IEEE Transactions on Ultrasonics, Ferroelectrics, and Frequency Control, Vol. 54, 2007, pp. 498-509.

[117] S. Deville, "Freeze Casting of Porous Ceramics: A Review of Current Achievements and Issues," Advanced Engineering Materials, Vol. 10, 2008, pp. 155-169.

[118] M. C. Gutterrez, M. L. Ferrer, F. del Monte, "Ice-Templated Materials: Sophisticated Structures Exhibiting Enhanced Functionalities Obtained after Unidirectional Freezing and Ice-SegregationInduced Self-Assembly," Chemistry of Materials, Vol. 20, 2008, pp. 634-648.

[119] H. Zhang, A. I. Cooper, “Aligned Porous Structures by Directional Freezing,” Advanced Materials, Vol. 19, 2007, pp. 1529-1533.

[120] H. Zhang, I. Hussain, M. Brust, M. Butler, S. Rannard, A. Cooper, "Aligned Two and Three Dimensional Freezing of Polymers and Nanoparticles," Nature Matereials, Vol. 4, 2005, pp. 787-793.

[121] M. C. Gutterrez, Z. Y. Garcia-Carvajal, M. Jobbagy, F. Rubio, L. Yusie, F. Rojo, M. L. Ferrer, F. del Monte, "Poly(vinyl alcohol) Scaffolds with Tailored Morphologies for Drug Delivery and Controlled Release," Advanced Functional Materials, Vol. 17, 2007, pp. 3505-3513.

[122] G. W. Oetjen, P. Haseley, “Freeze-Drying,” Berlin, Wiley, 2004.

[123] E. T. Angelakos, P. Bernardini, W. C. Barrett, "Myocardial Fibre Size and Capillary-Fibre Ratio in the Right and Left Ventricles of the Rat," The Anatomical Record, Vol. 149, 2005, pp. 671-676.

[124] K. R. Marutyan, M. Yang, S. L. Baldwin, K. D. Wallace, M. R. Holland, J. G. Miller, "The Frequency Dependence of Ultrasonic Velocity and the Anisotropy of Dispersion in Both Freshly Excised and 
Formalin-Fixed Myocardium," Journal of Ultrasound in Medicine and Biology, Vol. 32, 2006, pp. 603610.

[125] D. Recchia, C. S. Hall, R. K. Shepard, J. G. Miller, S. A, Wickline, "Mechanisms of the ViewDependence of Ultrasonic Backscatter from Normal Myocardium," IEEE Transactions on Ultrasonics, Ferroelectrics, and Frequency Control, Vol. 42, 1995, pp. 91-98.

[126] W. D. Obrien, J. E. Olerud, "Ultrasonic Assessment of Tissue Anisotropy," Proceedings of the International Ultrasonics Symposium, 1995, pp. 1145-1148.

[127] N. Kudo, T. Kamataki, K. Yamamoto, H. Onozuka, T. Mikami, A. Kitabatake, Y. Ito, H. Kanda, "Ultrasound Attenuation Measurement of Tissue in Frequency Range 2.5-40 MHz Using a MultiResonance Transducer," Proceedings of IEEE International Ultrasonics Symposium, 1997, pp. 11811183.

[128] M. Vogt, B. Paul, S. Scharenberg, R. Scharenberg, H. Ermert, "In Vivo Ultrasound Biomicroscopy of Skin with $20 \mathrm{MHz}$ and $100 \mathrm{MHz}$ Range Ultrasound: Inverse Echo Signal Filtering Optimization," Proceedings of the IEEE International Ultrasonics Symposium, Rotterdam, 2005. 


\section{Appendix - Full Circuit Schematics}

\section{A1 - 100 MHz Transmitter Circuit Diagram}
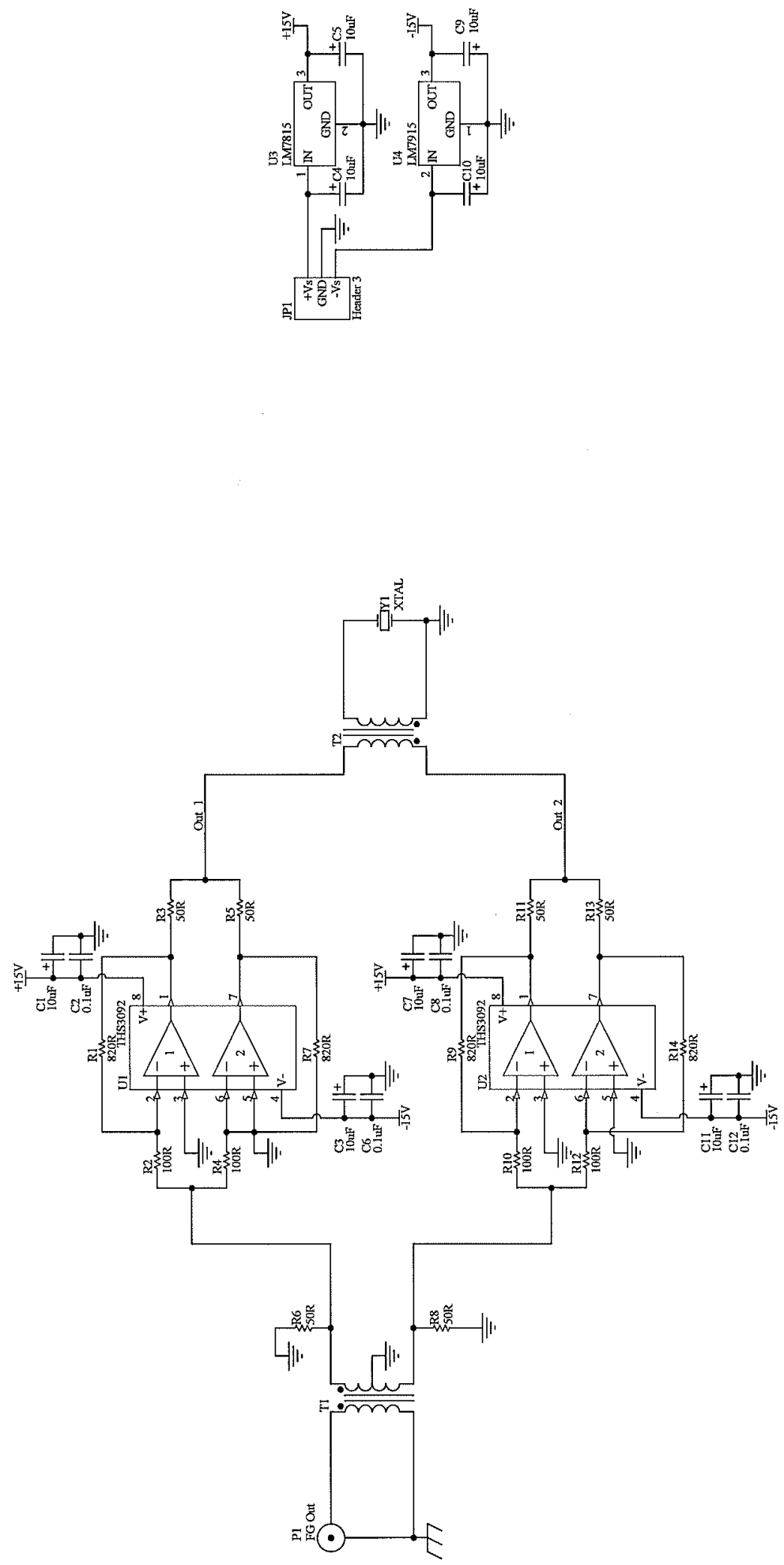


\section{A2 - 100 MHz Receiver Circuit Diagram}
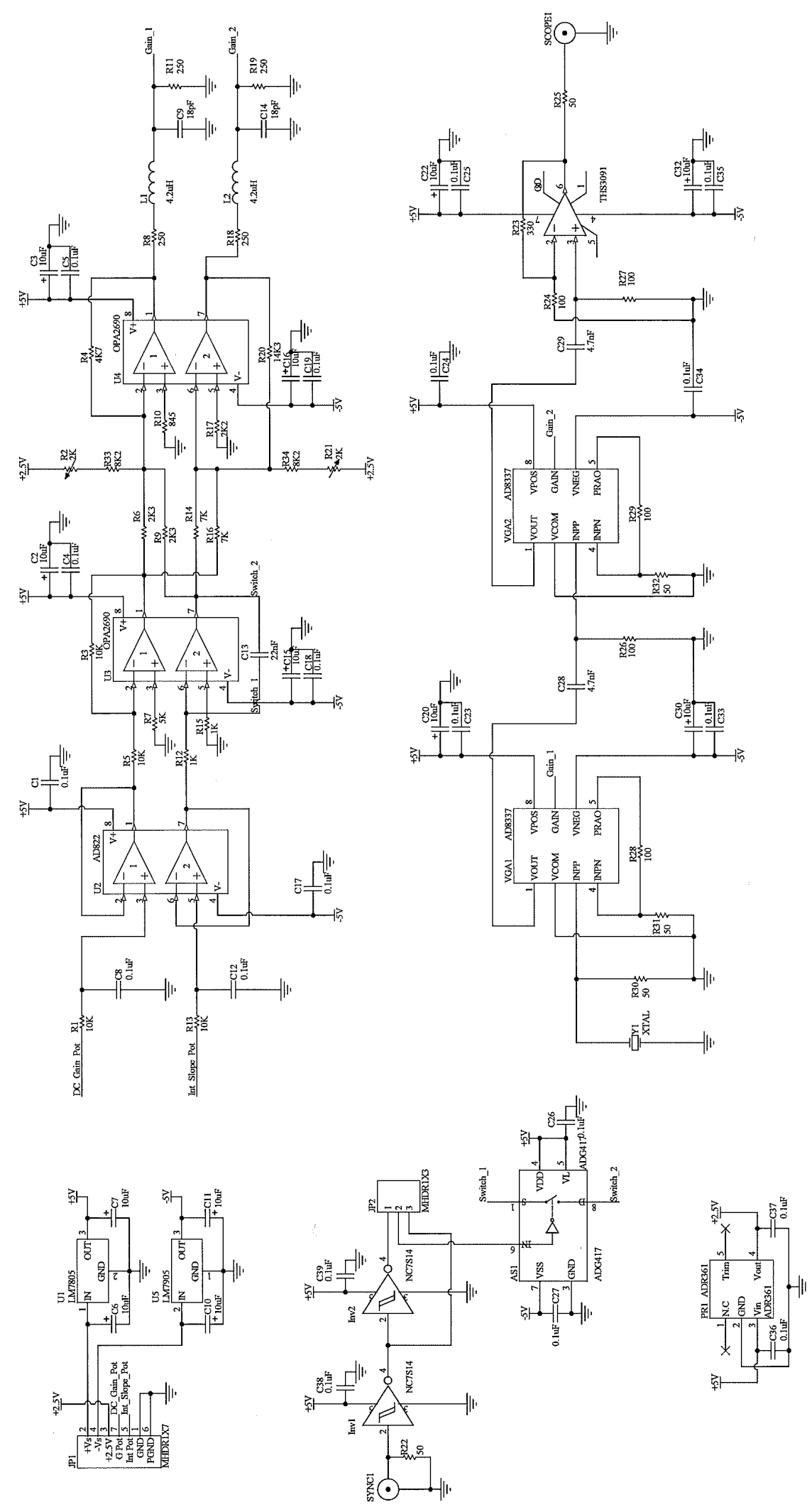


\section{A3 - 500 MHz Receiver Circuit Diagram}
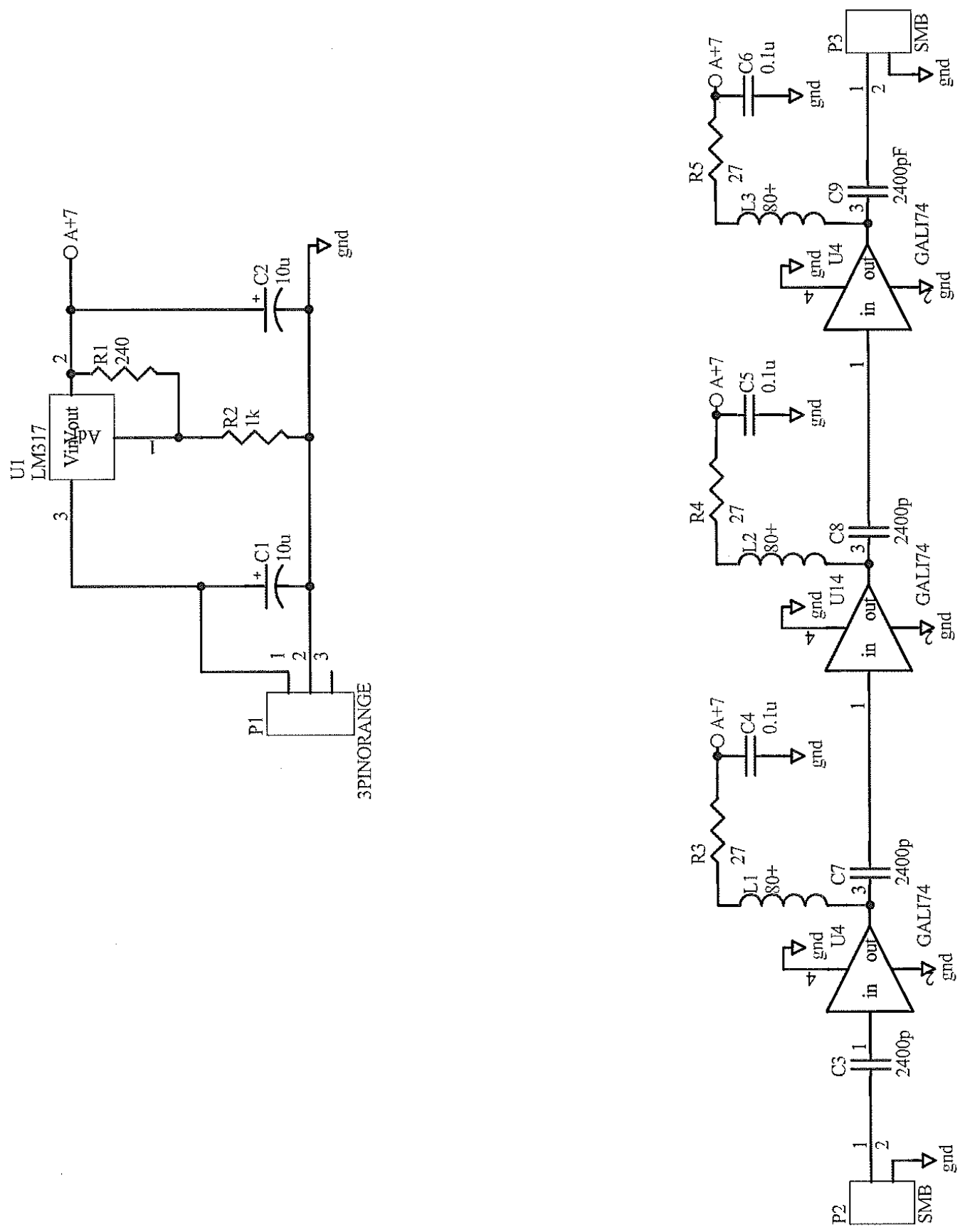

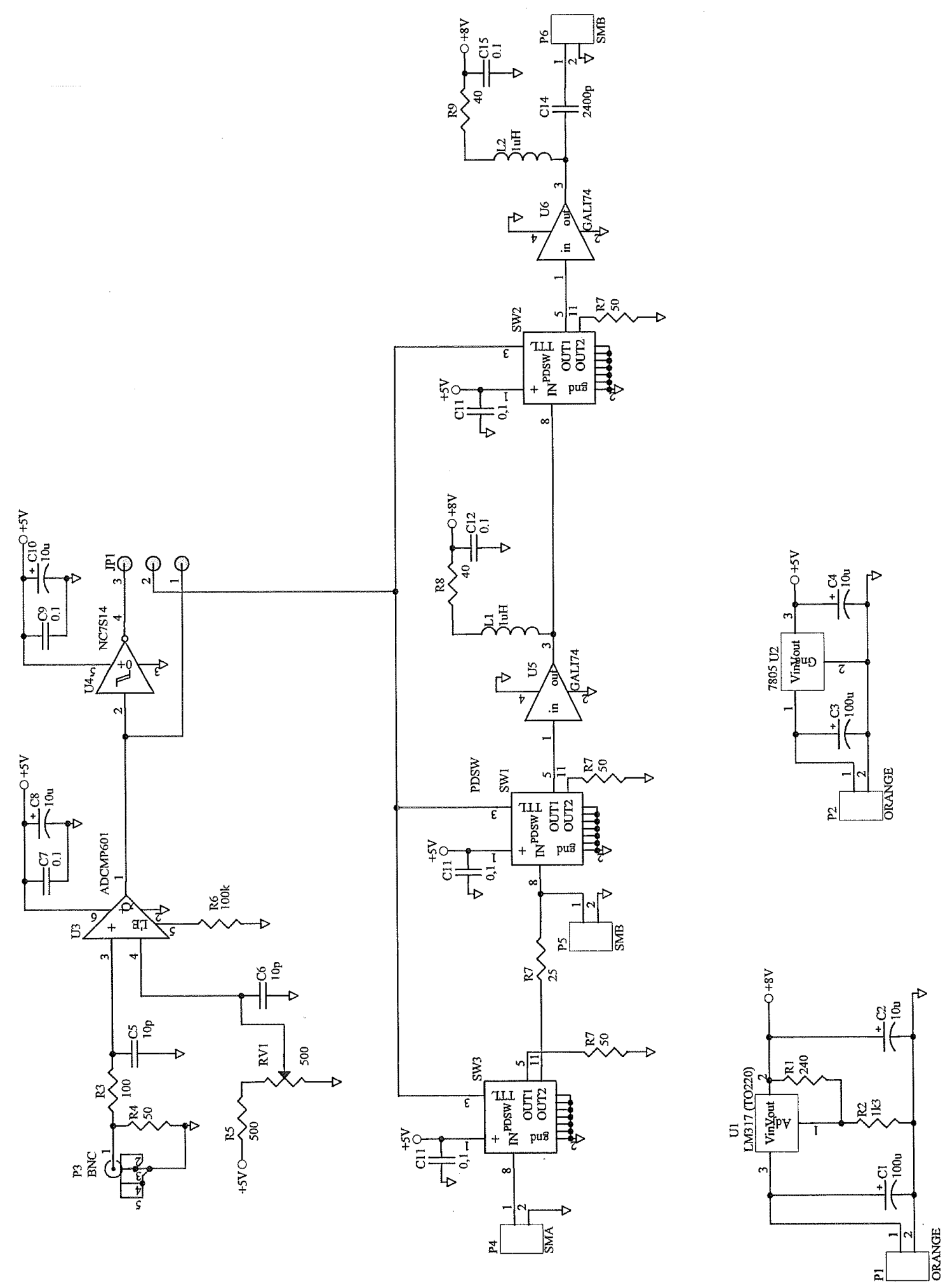
